LBL -31793

DE93 010417

\title{
Optical Pumping and Xenon NMR
}

\author{
M. Daniel Raftery \\ Department of Chemistry \\ University of California \\ and \\ Materials Sciences Division \\ Lawrence Berkeley Laboratory \\ University of California \\ Berkeley, California 94720
}

November 1991

This work was supported by the Director, Office of Energy Research, Office of Basic Energy Sciences, Materials Sciences Division of the. U.S. Department of Energy, under Contract No. DE-AC03-76SF00098.

\section{MASTER}




\title{
Optical Pumping and Xenon NMR
}

\author{
Copyright 두 1991
}

by

M. Daniel Raftery

The U.S. Department of Energy has the right to use this document for any purpose whatsoever including the right to reproduce all or any part thereof 


\section{Optical Pumping and Xenon NMR}

by

\section{Daniel Raftery}

Abstract

Nuclear Magnetic Resonance (NMR) spectroscopy of xenon has become an important tool for investigating a wide variety of materials, especially those with high surface area. The sensitivity of its chemical shift to environment, and its chemical inertness and adsorption properties make xenon a particularly useful NMR probe. This work discusses the application of optical pumping to enhance the sensitivity of xenon NMR experiments, thereby allowing them to be used in the study of systems with lower surface area.

A novel method of optically-pumping ${ }^{129} \mathrm{Xe}$ in low magnetic field below an NMR spectrometer and subsequent transfer of the gas to high inagnetic field is described. NMR studies of the highly polarized gas adsorbed onto powdered samples with low to moderate surface areas are now possible. For instance, NMR studies of opticallypumped xenon adsorbed onto polyacrylic acid show that xenon has a large interaction with the surface. By modeling the low temperature data in terms of a sticking probability and the gas phase xenon-xenon interaction, the diffusion coefficient for xenon at the surface of the polymer is determined. The sensitivity enhancement afforded by optical pumping also allows the NMR observation of xenon thin films frozen onto the inner surfaces of different sample cells. The geometry of the thin films results in interesting line shapes that are due to the bulk magnetic susceptibility of xenon.

Experiments are also described that combine optical pumping with optical detection for high sensitivity in low magnetic field to observe the quadrupolar evolution of ${ }^{131} \mathrm{Xe}$ spins at the surface of the pumping cells. In cells with macroscopic asymmetry, 
a residual quadrupolar interaction causes a splitting in the ${ }^{131} \mathrm{Xe}$ NMR frequencies in bare Pyrex glass cells and cells with added hydrogen.

Conventional xenon NMR experiments conducted on coadsorbed organic molecules in $\mathrm{Na}-\mathrm{Y}$ zeolites show that the xenon chemical shift is sensitive to the identity and concentration of these guests. Clusters of xenon trapped in Na-A zeolite have interesting statistical distributions due to the finite atomic size of the xenon. The statistics can be described in the low to moderate loading regime by binary and hypergeometric distribution functions.

Finally, a comprehensive review of the xenon NMR literature, including previous optical pumping studies, is provided. 


\section{Contents}

1 Introduction 1

1.1 Xenon Resonance Shifts ................... 3

1.2 Relaxation Times ................... 6

1.3 Optical Pumping ....................... 8

2 NMR Studies of Xenon 20

2.1 Gas Phase Experiments .................. 20

2.1 .1 Chemical Shifts ................. 20

2.1.2 Temperature Dependences . . . . . . . . . . . 21

2.1.3 Relaxation Studies . . . . . . . . . . . . . . 22

2.2 Xenon with Other Gases . . . . . . . . . . . . . . . 23

2.3 Solid and Liquid Xenon $\ldots \ldots \ldots$. . . . . . . . . . . . . 24

2.4 Xenon Thin Films and Clusters . . . . . . . . . . . . . 27

2.4 .1 Xenon on Graphite . . . . . . . . . . . . . 27

2.4 .2 Frozen Xenon Thin Films . . . . . . . . . . . 28

2.4.3 Clusters of Xenon in Na-A Zeolite . . . . . . . . . . . 28

2.5 Optically-Pumped Xenon Experiments .............. 31

$2.6{ }^{131} \mathrm{Xe}$ Optical Pumping Experiments . . . . . . . . . . . . . 32

3 Xenon as a Probe of Structure $\quad 37$ 
3.1 Low Surface Area Studies $\ldots \ldots \ldots \ldots \ldots \ldots$

3.2 Zeolites $\ldots \ldots \ldots \ldots \ldots \ldots \ldots \ldots \ldots \ldots$

3.2.1 Pure Zeolites: Na-Y, Na-A ZSM-5 and Others . . . . . . 40

3.2 .2 Metal Zeolites . . . . . . . . . . . . . . . . . . . . 45

3.2 .3 Exchanged Cations . . . . . . . . . . . . . 46

3.2.4 Low Temperature Studies . . . . . . . . . . . . . . 47

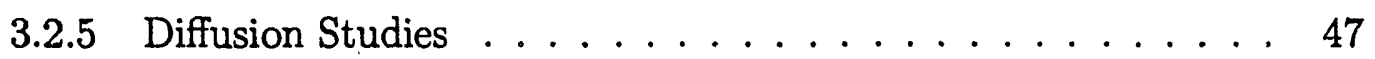

3.2 .6 Coking of Zeolites . . . . . . . . . . . . . . . 48

3.2.7 Zeolite-Guest Systems . . . . . . . . . . . . . . . . . 49

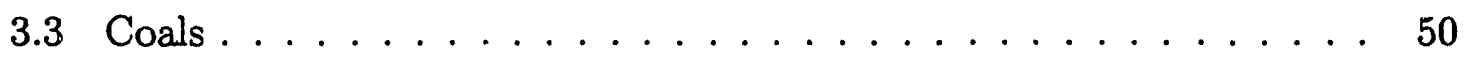

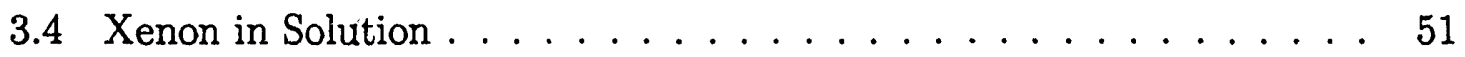

3.5 Clathrates . . . . . . . . . . . . . . . . 54

3.5.1 Cross-Polarization Experiments . . . . . . . . . 55

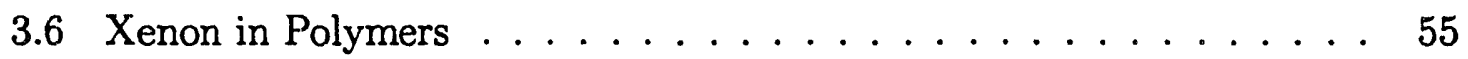

3.7 Xenon Compounds . . . . . . . . . . . . . . . 57

3.8 Xenon with Biological Molecules . . . . . . . . . . . . 58

4 Xenon as a Probe of Organic Molecules Adsorbed in Na-Y Zeolite 60

4.1 Sample Preparation and Data Collection . . . . . . . . . . 61

4.2 Results . . . . . . . . . . . . . . . . . . . 62

4.3 Discussion . . . . . . . . . . . . . . 68

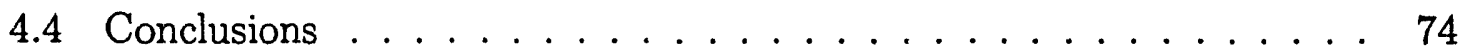

5 Measurement of Xenon Clusters in Na-A Zeolite $\quad 75$

5.1 Sample Preparation . . . . . . . . . . . . . . 76

5.2 Results . . . . . . . . . . . . . . . . . . 78

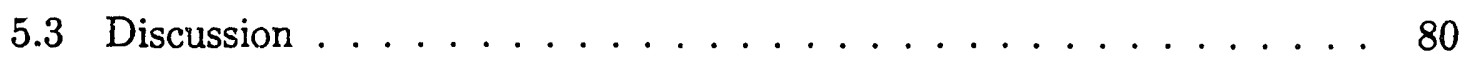


5.4 Conclusions . . . . . . . . . . . . . . . . . . . 84

6 Optically-Pumped Xenon Experiments in Low Magnetic Field 85

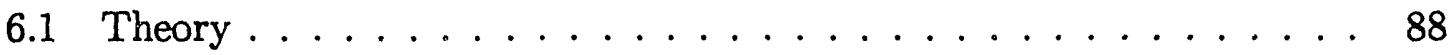

6.1.1 Optical Pumping and Relaxation ............ 88

6.1.2 Quadrupolar Interactions with the Wall . . . . . . . . 9 90

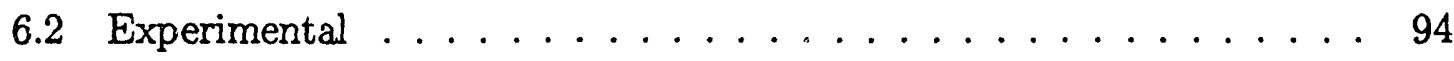

6.2.1 Cell Preparation . . . . . . . . . . . . . . 94

6.2 .2 Apparatus ....................... 96

6.3 Results and Discussion . . . . . . . . . . . . . . . . . 101

$6.3 .1{ }^{129} \mathrm{Xe}$ Nutation Experiments . . . . . . . . . . . . 101

6.3.2 ${ }^{131} \mathrm{Xe}$ Nutation Experiments . . . . . . . . . . . 101

6.3.3 Multiple Pulse Experiments . . . . . . . . . . . . 104

6.3.4 Increasing the Bandwidth .............. 104

6.3.5 Phase-Reversed Nutation Experiments . . . . . . . . . . 107

6.3.6 $\mathrm{T}_{1}$ Measurements ................... 109

6.4 Conclusions ............................ 111

7 Optically-Pumped Xenon Detected in High Field 112

7.1 Introduction . . . . . . . . . . . . . . . 112

7.2 Signal Enhancement: A Demonstration . . . . . . . . . . . . . . 113

7.3 Experimental Details . . . . . . . . . . . . . . . . . 115

7.3.1 The Apparatus . . . . . . . . . . . . . . 115

7.3 .2 NMR Probe . . . . . . . . . . . . . . 116

7.3.3 Timing Considerations . . . . . . . . . . . . . . 119

7.3.4 Recycling the Experiment . . . . . . . . . . . . 121

7.3.5 Sample Treatments . . . . . . . . . . . . . . . 122 
7.3.6 Water Contamination . . . . . . . . . . . . . 122

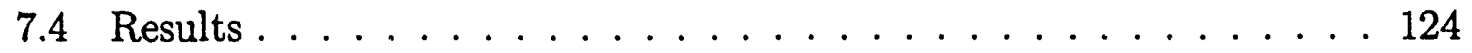

7.4.1 Benzanthracene . . . . . . . . . . . . . 124

7.4 .2 Na-Y Zeolite. . . . . . . . . . . . . 126

7.4 .3 Polyacrylic Acid Studies . . . . . . . . . . . . . 128

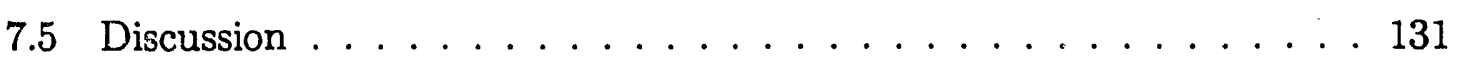

7.6 Conclusions ......................... 140

8 NMR of ${ }^{129}$ Xe Thin Films 141

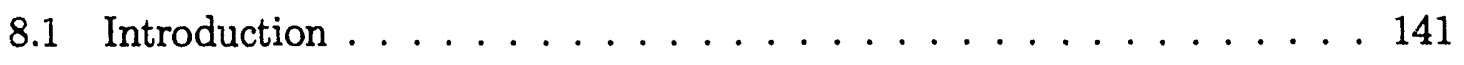

8.2 Theory ............................. 143

8.3 Experimental . . . . . . . . . . . . . . . 146

8.4 Results .......................... 147

8.5 Discussion ......................... 151

8.6 Conclusions .......................... 153

$\begin{array}{ll}\text { A Isotherms } & 154\end{array}$

A.1 Introduction . . . . . . . . . . . . . . 154

A.2 Gas Rack . . . . . . . . . . . . . . . . . 155

A.3 Polyacrylic Isotherms . . . . . . . . . . . . . . . 158

B Low Field Experimental Details $\quad 160$

B.1 Pulse Programmer ..................... 160

B.2 The Magnet ........................ 161

B.3 Magnet Supply . . . . . . . . . . . . . . . . 163

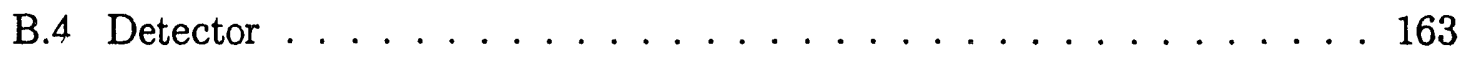

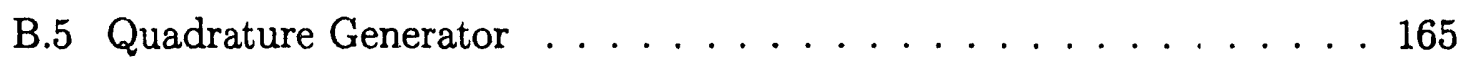


B.6 Rubidium Detection Lamp . . . . . . . . . . . . . 165

Bibliography 


\section{Acknowledgments}

It is a pleasure to acknowledge the many people who have contributed to my graduate experience here at Berkeley. My years in the Pines group have been many, but not without their benefits in terms of science, experience and social life.

As a beginning graduate student, I was convinced by Alex's enthusiasm and personality, perhaps more than any other factors, to join his research group. More and more I come to realize that his ability to combine first rate science with style and excitement is unique and a strong driving force behind the Pines group.

As for my co-workers, I am forever thankful for their assistance and advice in getting my somewhat unusual and sometimes frustrating project off the ground. I will always remember Dave Shykind's enthusiasm, late night antics and unending risqué jokes during my first two years. Henry Long has been an excellent co-worker, and I look forward to his research efforts in furthering the project. Linda Reven and Phil Grandinetti have injected much needed expertise to the project at critical times, and Thomas Meersmann, Pei Tang and Dave Anvar are also gratefully acknowledged for their much needed assistance.

Shang-Bin Liu and Louis-Charles "Kiko" de Menorval are appreciated for their advice and humor auring my early graduate days. Kiko was also a gracious host during my stay at his castle in Britany. I enjoyed working and pursuing our mutual interests in running and listening to Jazz with Brad Chmelka. I would also like to thank Herman Cho, Chuck Conner, and Steve Rucker for their fine theoretical and technical help.

Aside from his valuable advice and teaching, I thank A.J. Shaka for the many late afternoons fighting the "connector" along the Strawberry Canyon trail. Happily, A.J. has hit the peak and kept on climbing. I hope to rejoin him on the trail soon.

I would also like to thank Roberta and Dave Drewry, Charlie and Karen Goss 
and Terry Franklin for some very amusing times during the past several years. As for diversions, I would like to thank the "cool Jazzers" at the U.C. Jazz Ensembles, and especially my teachers Ann Tappari and Susan Muscarella for providing an outlet when I needed to escape the lab.

Most importantly, I thank my parents, Jennifer, Johnny, the rest of my family and Ana for their love and encouragement. 


\section{Chapter 1}

\section{Introduction}

Xenon has a variety of interesting properties which have made it important in disciplines from Physics to Biochemistry. Xenon has become an important nucleus for nuclear magnetic resonance (NMR) experiments, primarily due to the sensitivity of xenon's NMR parameters to its environment, and xenon's chemical inertness, which allow it to probe a variety of interactions. The large electron cloud polarizability of xenon leads to its facile adsorption properties and its enormous resonance (chemical) shift range. Two isotopes exist that are NMR accessible: spin $1 / 2{ }^{129} \mathrm{Xe}$, which comprises the vast majority of the xenon NMR literature; and spin $3 / 2{ }^{131} \mathrm{Xe}$, which has a quadrupolar moment. Interactions of xenon with various materials have been used to look at the chemistry of materials and the physics of xenon's interactions with them. The first three chapters review recent aspects of xenon NMR of interest to a general chemistry audience. Both conventional and optical pumping NMR experiments will be discussed, including xenon's applications to a wide variety of materials. The review will focus on the physics and chemistry of xenon's interactions with its environment, other xenon atoms, various ligands in xenon compounds [1], and with other species or materials in the gas, liquid and solid phases. I will also try 
to outline future directions for xenon NMR when possible and contrast experiments from an array of different fields of science.

Chapter 1 briefly reviews the relevant NMR parameters used in the study of xenon such as resonance shifts, line widths, line shapes, and relaxation times. Familiarity with the basics of NMR are assumed, and the reader is referred to the many excellent texts on the subject. A section describing the technique of optical pumping to enhance the polarization of xenon nuclear spin discusses its salient features. Chapter 2 focuses on investigations of xenon itself in the gas, liquid and solid phases in terms of these relevant NMR parameters. Theory and experimental results are well, but not completely, matched indicating that some complexities remain to be resolved. I discuss xenon's application to the study of materials in Chapter 3 , including experiments on materials such as zeolites, silicates, liquid crystals and biological molecules. These data give insight to the types of interactions that xenon experiences as well as the symmetry or geometry of xenon's environment. Applications of xenon NMR have become more prevalent after the discovery $[2,3]$ that xenon could be used to determine structural aspects of materials with large surface areas which absorb xenon readily, the most important being zeolites. This is a growing field driven mainly by the interest in determining structural aspects of these materials, possible catalytic properties and guest/host interactions. This material has recently been reviewed, [4] so the discussion is brief. Recent experiments on low surface-area, powdered materials that are now accessible to xenon NMR using methods of optical pumping [5] are also discussed.

After the review of xenon NMR, experiments performed in the Pines lab are discussed in detail. Conventional xenon NMR experiments are described in the next two chapters. Xenon's ability to detect coadsorbed organic guest molecules in $\mathrm{Na}-\mathrm{Y}$ zeolite is considered in Chapter 4. Xenon clusters formed at high temperature and 
high pressure in Na-A zeolite are described in Chapter 5 where the distribution of the clusters shows the influence of the finite atomic size of the xenon. atoms inside the micropores of the zeolite structure. Chapter 6 concerns optical pumping experiments carried out in low magnetic field where optical detection is also employed to achieve high sensitivity from low pressure gas samples of ${ }^{129} \mathrm{Xe}$ and ${ }^{131} \mathrm{Xe}$. Surface interactions of quadrupolar ${ }^{131} \mathrm{Xe}$ in asymmetric cells are studied using optical pumping and optical detection in low magnetic field. Chapter 7 represents the bulk of my research time spent at Berkeley and the realization of the original goal of the xenon optical pumping work. The chapter concerns the optical pumping of xenon in low magnetic field and subsequent transport of the gas and its detection by NMR in high magnetic field. Data from studies on several surfaces using this new technique are discussed, including a full study of polyacrylic acid where the surface interaction is analyzed in terms of xenon's diffusion on the surface. Another application of the technique is discussed in Chapter 8 where optical pumping is used to enhance the NMR signal of thin films of xenon, and the effect of the anisotropic bulk magnetic susceptibility can plainly be seen for different film geometries. Finally, some concluding remarks can be found in Chapter 9. First, however, a short discussion of the NMR parameters relevant to the study of xenon NMR is presented.

\subsection{Xenon Resonance Shifts}

Probably the most unusual and important NMR parameter of ${ }^{129} \mathrm{Xe}$ is its resonance (chemical) shift that has an enormous range of over $7500 \mathrm{ppm}$ (parts per million). Xenon's large and extremely polarizable electron cloud make its NMR chemical shift a very sensitive measure of xenon's interactions with its environment. As discussed by Ramsey, [6] the chemical shift is composed of two parts, diamagnetic (originally 
derived by Lamb [7]) and paramagnetic shifts, that have opposite effects on the nuclear resonance frequency. The chemical shift may be written:

$$
\sigma=\frac{e^{2}}{3 m c^{2}} \int \frac{\rho}{r} d \tau-\frac{4}{3 \Delta E}\left\langle 0\left|\sum_{j, k} L_{j} \cdot \frac{L_{k}}{r_{k}^{3}}\right| 0\right\rangle
$$

where $\rho$ is the electron density, $\Delta \mathrm{E}$ is the average excited-state energy, $L_{j}$ is the angular moment operator, and $r_{k}$ is the electron-nuclear distance. Diamagnetic shifts occur from the interaction of the electron orbitals with applied magnetic fields. The electrons are caused to precess in the applied field in such a way as to oppose the applied field (Lenz's law). This effect is said to shield the nucleus from the applied field, reducing the resonance frequency of the nucleus. The diamagnetic term has been calculated for isolated atoms $[8,9]$ and for xenon, it is about $5600 \mathrm{ppm}$ to lower frequency relative to the bare nucleus. In Figure 1.1, ${ }^{129} \mathrm{Xe}$ chemical shifts are shown for a variety of different xenon interactions: pure xenon phases, xenon compounds, and xenon in different environments. Gas phase, atomic xenon at low pressure is defined as having a chemical shift of zero as a reference.

More important to chemistry are the paramagnetic shifts that arise from excited electronic state mixing and that increase the resonance frequency of the xenon nucleus. The paramagnetic term gives rise to the observed resonance shift in interactions of xenon with itself (pressure-dependent shift $[10,11])$ and other species. Paramagnetic terms can cause shifts more deshielded that the bare nucleus in xenon compounds such as $\mathrm{XeO}_{6}^{-4}[1]$, or more shielded (negative chemical shift) than atomic xenon itself [12]! Calculations of the paramagnetic shifts are more difficult, especially those involving molecular bonds where two-centered (or more) interactions must be considered. The choice of reference frame for calculations (which is equivalent to the choice of gauge) is an important consideration for it actually changes the way the paramagnetic and diamagnetic terms comprise the chemical shift [13]. 


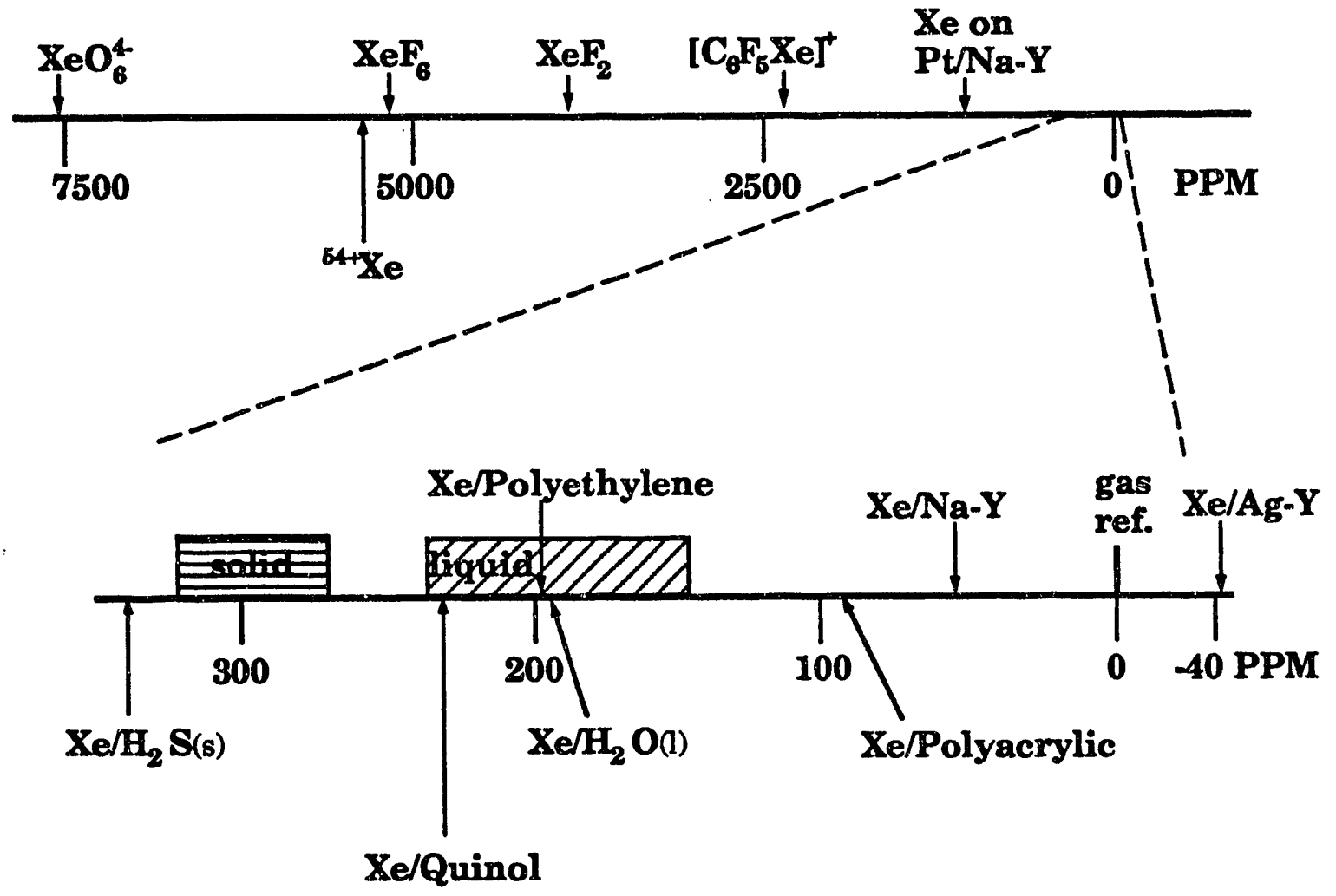

Figure 1.1: ${ }^{129} \mathrm{Xe}$ NMR chemical shift scale and several representative xenon compounds, phases, and environments. The scale has been expanded at the low end to show the phase-dependent chemical shifts. 


\subsection{Relaxation Times}

The relaxation times, $T_{1}$ and $T_{2}$ are also important xenon NMR parameters. As originally discussed by Bloembergen [14], $T_{1}$ relaxation times are governed by the interactions of the nuclear spin with the random energy fluctuations of the environment (collectively called the "lattice") at the resonance frequency of the nuclear spin. The lattice is responsible for causing spin-flips with a random probability that act to bring the nuclear spin system to an equilibrium state that can be described by a Boltzmann distribution at the lattice temperature in a characteristic time $T_{1}$

Random fluctuations of some perturbation to the spin system, such as a magnetic field $\mathrm{H}$, are described with a correlation function that has an exponentially decaying form:

$$
\overline{H(T) H(T+t)}=H^{2} e^{-t / \tau_{c}} .
$$

Fourier transformation gives the frequency response of the relaxation rate [15]:

$$
\frac{1}{T_{1}}=2 \gamma^{2} H^{2} \frac{\tau_{c}}{1+\omega^{2} \tau_{c}^{2}}
$$

where $\tau_{c}$ is the correlation time and $H_{\circ}$ is a random, fluctuating magnetic field.

Spin-spin relaxation times, $T_{2}$, are similar in form to that for $T_{1}$, except that fluctuations at zero frequency also contribute to $T_{2}$ relaxation. Energy conserving flip-flops of dipolar-coupled spins such as found in solids cause rapid $T_{2}$ relaxation, for instance.

In Figure 1.2, data are shown for xenon relaxation times under a variety of conditions of temperature, and pressures. The well known features of the Figure are due to the fact that slow motions such as those found in solids (or at low temperatures) lead to long relaxation times and broad lines (short $T_{2}$ ), while fast motions as found in the gas or liquid phases also lead to long $T_{1}$ 's but to narrow lines. The $T_{1}$ mini- 


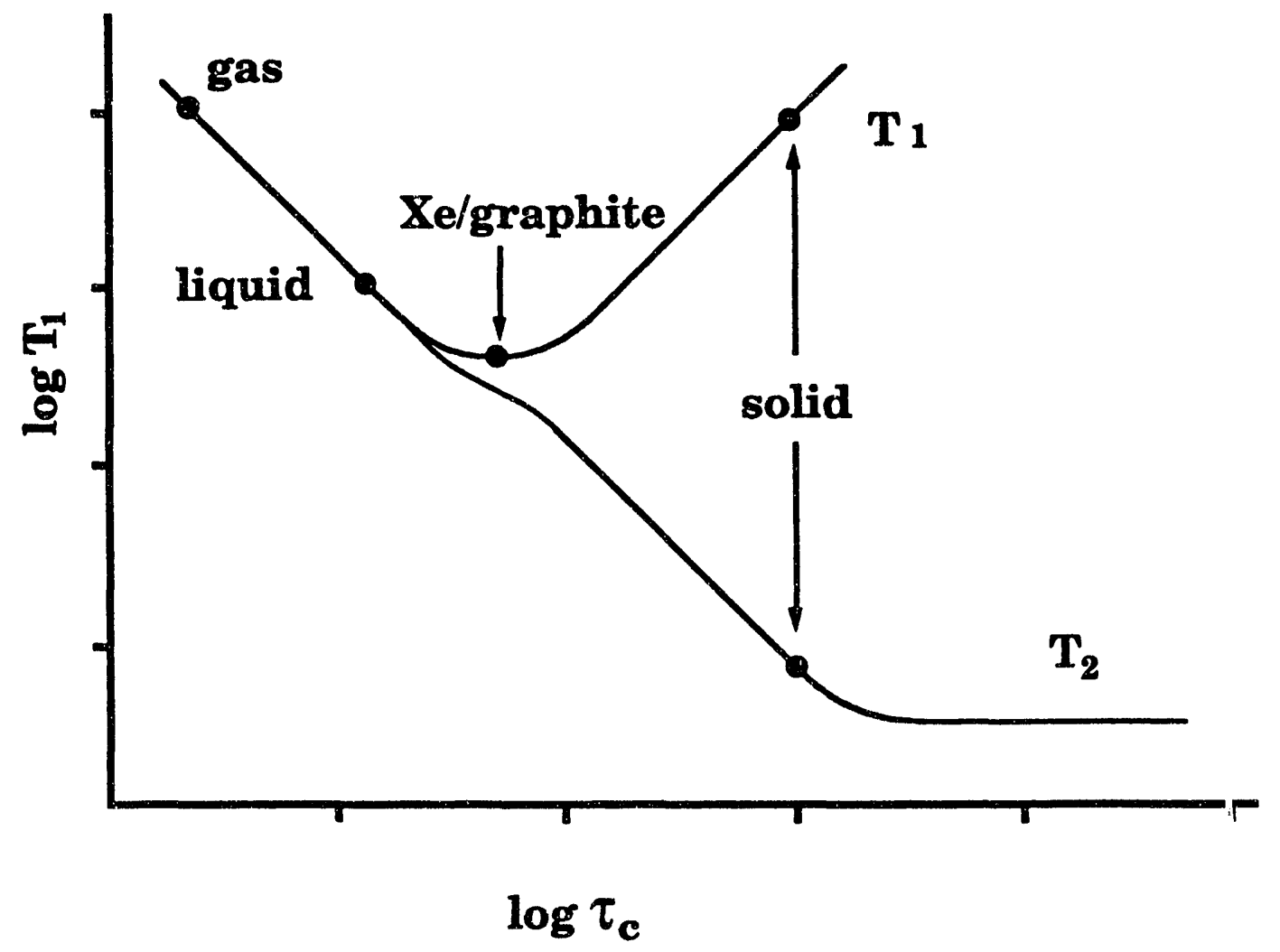

Figure 1.2: $T_{1}$ and $T_{2}$ relaxation times versus correlation time $\tau_{c}$ as discussed in reference [14]. Representative xenon NMR experiments are shown in their appropriate region of the plot. See text for details. 
mum occurs where the correlation time (collision time for gas phase) is equal to the Larmor period, $\tau_{c} \omega=1$.

As examples, solid xenon is located in the slow-motion regime, gaseous and liquid xenon are in the fast motion (which is also termed the motionally-narrowed regime), and xenon on graphite is near the $T_{1}$ minimum.

\subsection{Optical Pumping}

In a typical NMR experiment for a spin $1 / 2$ nucleus, the signal strength is mainly determined by the difference of the populations $P_{i}$ of the two energy states, whose ratio is called the Boltzmann factor:

$$
\frac{P_{2}}{P_{1}}=e^{-\left(E_{2}-E_{1}\right) / k T}=e^{-\gamma \hbar H / k T}
$$

At room temperature the ratio is about $10^{-5}$ or $10 \mathrm{ppm}$ for moderate magnetic strengths (4 Tesla) so that the polarizations are very small. This is not a problem for abundant nuclei such as protons. Many methods have been devised to increase the signal from less abundant or low frequency nuclei, including low temperature experiments or cross polarization [16] from highly polarized nuclei such as protons to less polarized nuclei.

Optical pumping provides a way to dramatically increase spin polarization. Discovered by Kastler in 1949 [17] (for which he later received the Nobel prize), it has become important in the study of gas phase spin interactions and the production of highly polarized spin reservoirs for use as high energy particle physics targets and for use in NMR experiments. The methods used in optical pumping experiments have been reviewed $[18,19,21]$.

In Figure 1.3, the energy levels for the valence electron of an alkali metal atom, such as rubidium are shown. Rubidium has an $S_{1 / 2}$ ground state that is split into two 

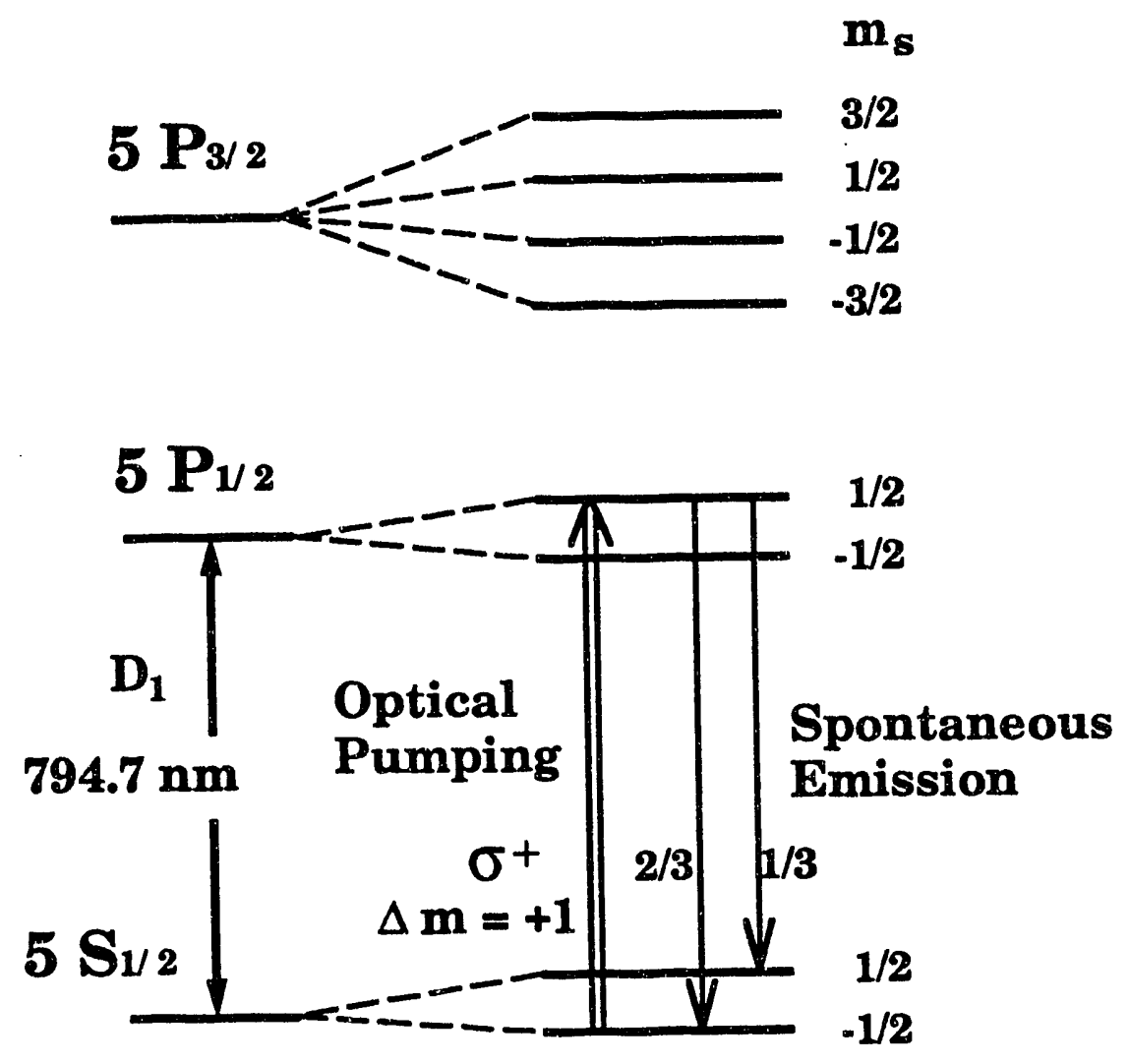

Figure 1.3: Energy levels of $\mathrm{Rb}$ excluding the nuclear spin. Right circularly polarized light at $754.7 \mathrm{~nm}$ causes transitions from the $\mathrm{m}_{s}=+1 / 2$ ground state to the $\mathrm{m}_{s}=-1 / 2$ excited state. Emission back to the ground state results in population built up in the $\mathrm{m}_{s}=-1 / 2$ ground state. 
levels in a magnetic field, neglecting hyperfine structure (rubidium's nuclear spin). The first two excited states, $P_{1 / 2}$ and $P_{3 / 2}$, also shown in the figure are also split by the magnetic field into two and four levels, respectively. Right circularly-polarized light $\left(\sigma^{+}\right)$, with a wavelength of 7947 Aand in the direction of the magnetic field, causes a transition from the ground state to the $P_{1 / 2}$ excited state with the selection rule of $\Delta m_{s}=+1$. This has the effect of depopulating the $\mathrm{m}=-1 / 2$ ground state. The excited state decays within about $30 \mathrm{nsec}$ (due to spontaneous emission) back to the two ground states with relative probabilities $1 / 3$ and $2 / 3$ given by the ClebschGordon coefficients. At the reasonably high pressures used in typical experiments, excited rubidium atoms collide before de-excitation which causes the excited states to mix so that the depolarization rates become $1 / 2$ and $1 / 2$. The net result of the optical pumping cycle is to leave one of the ground state levels highly polarized, since the relaxation time of the ground state is relatively long (up to 1 second).

Methods first introduced by Grover at Litton Industries [20] and later refined by Happer and coworkers [21] at Princeton showed that optical pumping could be used to dramatically increase and observe the nuclear polarization signal of xenon, and thereby allow its study under a variety of new and interesting conditions. During collisions with rare gas atoms, spin polarization may be transferred from the alkali metal atom to the rare gas nucleus. This "spin exchange", as it is called, is more efficient for large rare gas atoms like xenon because of its tendency to form long-lived van der Waals molecules [22], and the fact that the spin exchange efficiency varies as the interaction time squared. A schematic figure of a van der Waals molecule is shown in Figure 1.4.

The Hamiltorian during the van der Waals collision may be written:

$$
\mathcal{H}=A \mathbf{I} \cdot \mathbf{S}+\gamma \mathbf{N} \cdot \mathbf{S}+\alpha \mathbf{K} \cdot \mathbf{S}+\omega_{S} \mathbf{S}_{z}+\omega_{K} \mathbf{K}_{z}
$$




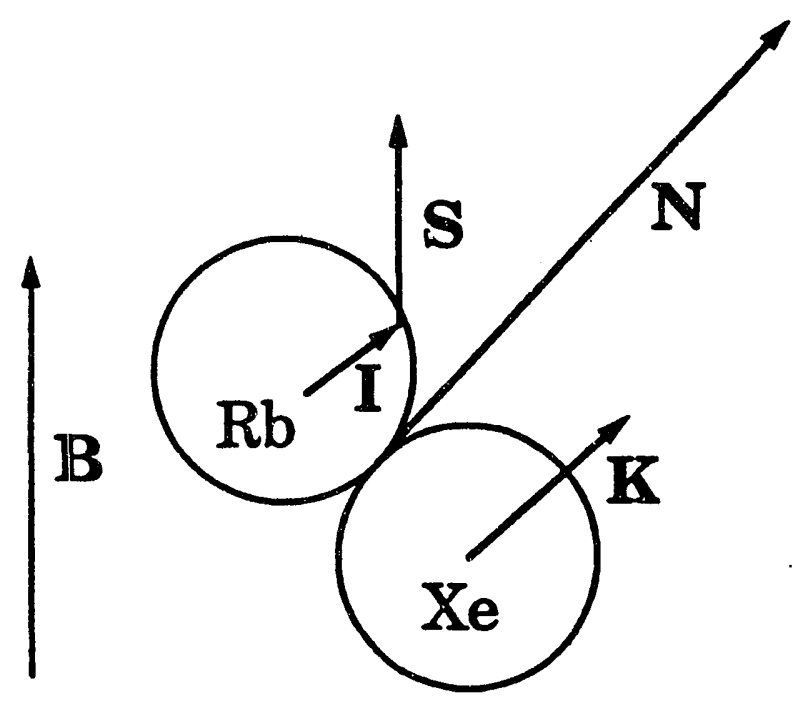

Figure 1.4: Schematic rubidium-xenon van der Waals complex, including rubidium nuclear spin $\mathbf{I}$, which is strongly coupled to the electron spin $\mathbf{S}$, xenon nuclear spin $\mathbf{K}$, and rotational angular momentum $\mathbf{N}$. 
where $\mathrm{A}$ is the rubidium hyperfine interaction, $\gamma$ is the spin rotation interaction coupling the rubidium electron spin to the van der Waals complex rotation, $\alpha$ is the Fermi contact term coupling the rubidium electron with the xenon nuclear spin $\mathbf{K}$, plus Zeeman terms.

The hyperfine interaction in ${ }^{85} \mathrm{Rb}$ is approximately $3 \mathrm{GHz}$ so the rubidium valence electron remains strongly coupled to the nucleus unless the magnetic field is above one Tesla or so (Pashen-Back effect). The electron-nuclear coupling has no affect on the optical pumping, but has the effect of reducing the Larmor frequency of the electron in a magnetic field by a factor of $1 /(2 I+1)$, where $I$ is the nuclear spin, and thus complicates the rubidium spin level system. This can lead to some interesting effects such as optically detected sublevel coherences experiments [23] and multiple quantum transitions [24] as well as the possibility of creating higher-order multipole polarizations [21], that have been recently reviewed by Suter and Mlynek [25].

The Fermi contact interaction, given by

$$
\alpha=\frac{8 \pi}{3} g \beta g_{N} \beta_{N} \delta(\mathbf{r}) \mathbf{I} \cdot \mathbf{S},
$$

is responsible for the spin exchange mechanism which transfers its polarization from rubidium to xenon. $\mathrm{g}$ and $\mathrm{g}_{N}$ are the electron and nuclear $\mathrm{g}$-factors, $\beta$ and $\beta_{N}$ are the electron and nuclear magnetic moments and $\delta(\mathbf{r})$ is the probability of finding the electron at the nuclear coordinate. Usually, the electron and nucleus are associated with the same atom, so that $r=0$. For Rb-Xe spin exchange, however, it is the probability of finding the $\mathrm{Rb}$ electron at the xenon nucleus which is important.

The spin coordinate term $\mathbf{I} \cdot \mathbf{S}$ can be rewritten:

$$
\mathbf{I} \cdot \mathbf{S}=\left(I_{x} S_{x}+I_{y} S_{y}\right)+I_{z} S_{z}=I^{+} S^{-}+I^{-} S^{+}+I_{z} S_{z}
$$

where $I^{+} S^{-}+I^{-} S^{+}$constitute the "flip-flop" terms that allow spin exchange. Schemat- 


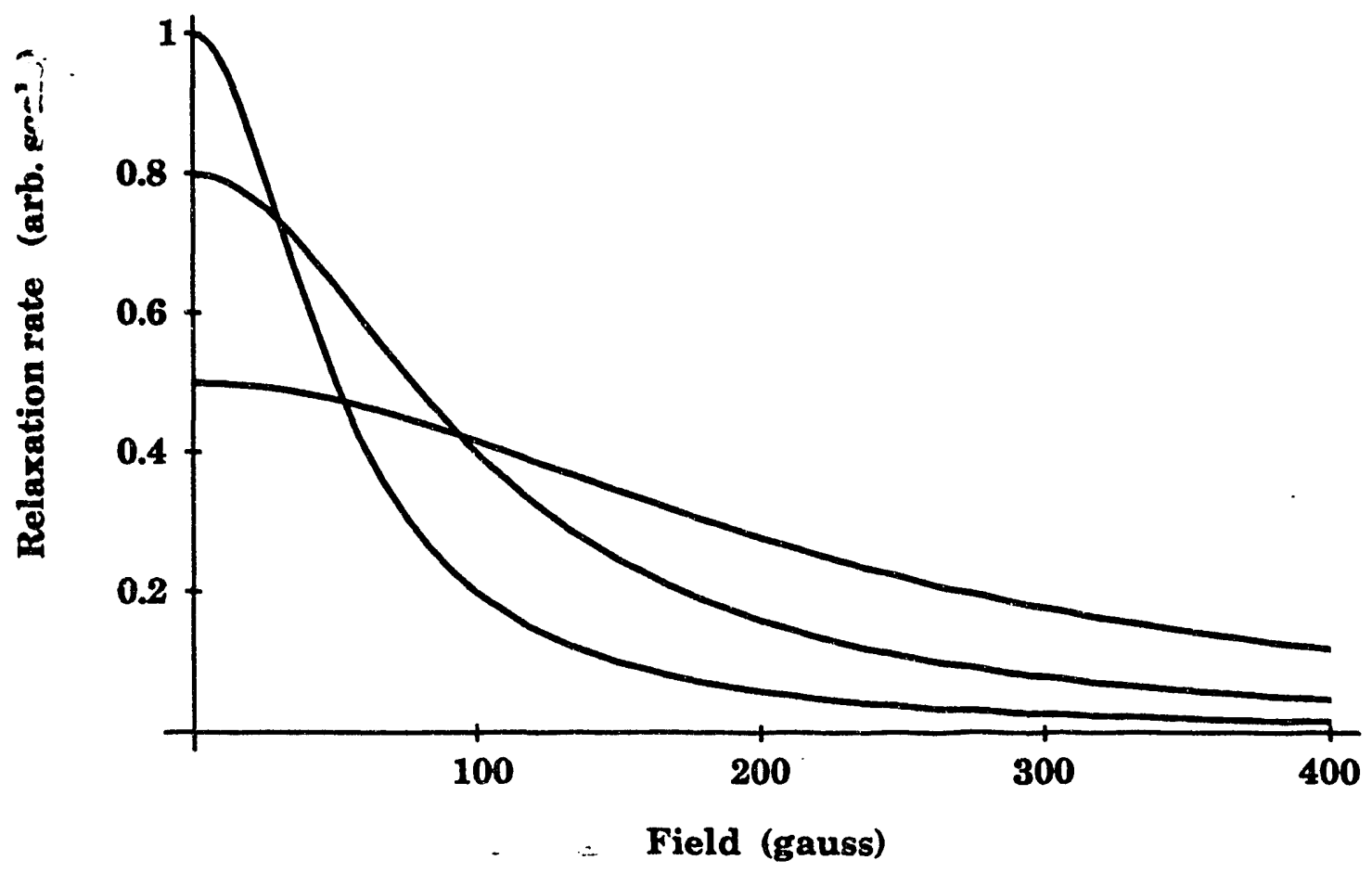

Figure 1.5: Rubidium relaxation rate slowing down curves. As the field is increased, the rubidium electron no longer couples to $\mathbf{N}$, the van der Waals complex rotation number. At higher pressures, the complex life time is shortened and the decoupling is less effective for the same value of the magnetic field. 
ically, one can think of the spin exchange as the following process:

$$
S(\uparrow)+I(\downarrow) \Rightarrow S(\downarrow)+I(\uparrow)
$$

As shown by Herman [27], the Fermi contact interaction is enhanced in the rubidium-xenon complex due to the electron exchange interaction between the rubidium $5 s$ electron and the core $s$ orbitals of xenon. The enhancement is on the order of $10^{3}$ which can be derived by writing a Slater determinant for the rubidium valence electron with the xenon $1 s$ orbitals:

$$
\psi=\left|\begin{array}{l}
R b(5 s) X e\left(1 s^{1}\right) X e\left(1 s^{2}\right) \\
R b(5 s) X e\left(1 s^{1}\right) X e\left(1 s^{2}\right) \\
R b(5 s) X e\left(1 s^{1}\right) X e\left(1 s^{2}\right)
\end{array}\right|
$$

the result being that the Fermi contact term is modified by a factor $\eta^{2}$, with:

$$
\eta=1-\sum_{i=2}^{n} \frac{\langle i \mid 1\rangle \delta_{i}(r)}{\delta_{1}(r)}
$$

where $\langle i \mid 1\rangle$ are overlap integrals, and $\delta_{i}(r)$ is the amplitude of the $i$ th orbital at the xenon nucleus. The spin exchange probability is proportional to $\eta^{4}$ which is about 3500 for xenon.

The spin-rotation interaction $\mathbf{N} \cdot \mathbf{S}$, acts as the primary relaxation mechanism for rubidium, and is constitutes a major loss pathway in the attempt to create high polarizations of xenon. The electron spin polarization is coupled to the rotational angular momentum of the van der Waals complex, which is then lost upon breakup of the complex. For $\mathrm{Rb}-\mathrm{Xe}$ complexes, the rotation number $\mathrm{N}$ is about 70 , and can be thought of as a classical vector [26]. At moderate magnetic fields, the rubidium electron may be decoupled from the spin-rotation interaction as measured by an increased relaxation time for rubidium [28](See Figure 1.5).

In Figure 1.6, a simplified apparatus for xenon optical pumping experiments. A laser tuned to the rubidium D1 transition (7947 $\AA$ ) illuminates the pumping cell for 
several minutes. Rubidium lamps are also capable of providing good polarizations. After pumping, the decay of the polarization can be monitored by simply measuring the change in transmission of rubidium light of a low power probe beam through the cell. Highly polarized rubidium vapor does not absorb the circularly polarized photons so that the detected light initially has a high intensity. As the spin polarization decays, the detection light is attenuated. There are many detection mechods used in optical pumping experiments that have very high sensitivity because they detect optical photons with essentially $100 \%$ efficiency. A series of crossed-beam pump and probe experiments have been described in the literature to observe a variety of phenomena, such as: alkali metal-atom spin-relaxation [22]; rubidium to cesium spin polarization transfer, both on resonance [29] and off resonance [30]; and $\mathrm{Hg}$ dephasing experiments [31]. For optically-pumped rare gas experiments, the long relaxation times allow one to pump and detect in the same, $z$ direction, by changing from the pump laser to a detection lamp, or by simply removing the optical polarizers [32]. Some experiments switch the magnetic field direction to observe transverse decays [33]. As shown in Figure 1.7, an unpolarized detection beam consisting of equal numbers of left and right circularly-polarized photons impinges on a cell containing polarized alkali metal vapor, and as a result, picks up a small polarization due to the difference in absorption for left and right circularly polarized light (optical birefringence). The polarized portion of the light can then be modulated by a Photo-Elastic Modulator (PEM) [34] and detected with a lockin amplifier with very high sensitivity. Note that in the early crossed-beam experiments the effect of the transverse field was simply to modulate the polarized light because of the evolution of the electron spin in the magnetic field. The modulation frequency was at the Larmor frequency $\omega=\gamma B_{\circ}$ of the unpaired electron (approximately $700 \mathrm{kHz} /$ gauss for ${ }^{85} \mathrm{Rb}$ ).

The polarization cycle can be seen in Figure 1.8 where the pumping light has 


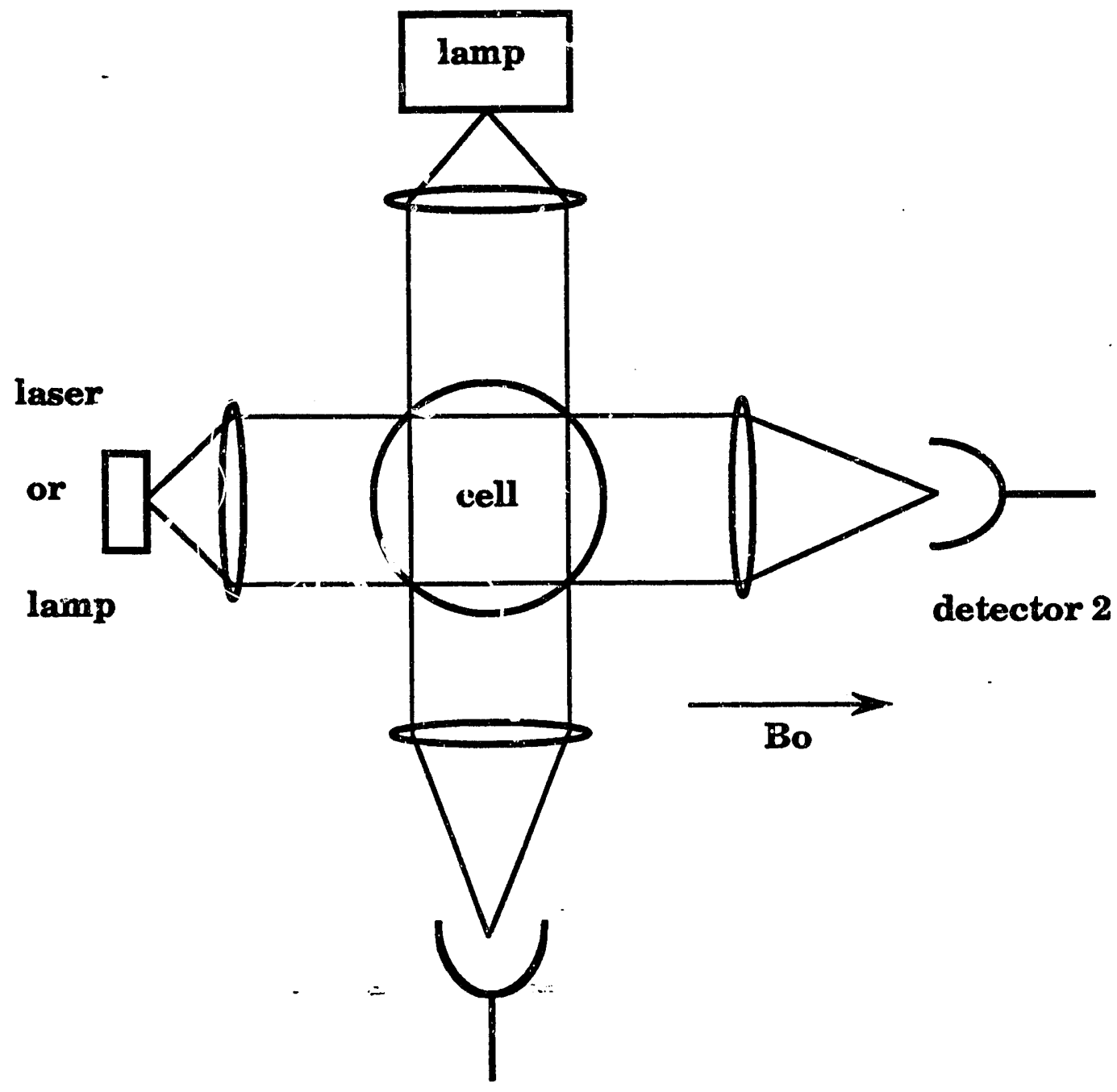

detector 1

Figure 1.6: A simplified schematic of a typical optical pumping apparatus. The pumping light (a laser) is incident in the $z$ direction while the detection light (unpolarized rubidium lamp light) can be incident along the $z$ direction, or in the transverse direction. 


\section{Bo}

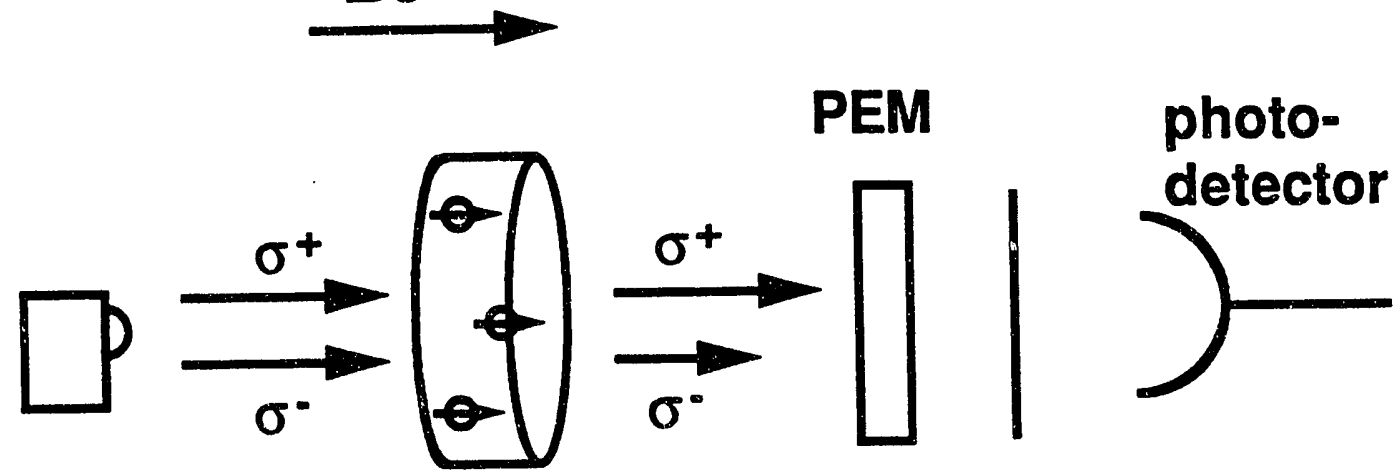

Rb lamp

cell

polarizer

Figure 1.7: Schematic diagram of the optical detection used in low field optical pumping experiments. Unpolarized rubidium light incident on polarized rubidium in the optical cells induces a birefringence in the light. The PEM (photo-elastic modulator) modulates this residual polarization in the light which may then be detected by a silicon photo detector and lockin amplifier. 
been turned on at time $t=0$. The rubidium polarization builds up quickly to a value approaching $100 \%$ and is given by [35]:

$$
P_{R b}=\frac{\Gamma / 2}{\Gamma / 2+\gamma_{x e}+\gamma_{w a l l}}
$$

where $\Gamma$ is the pumping rate of the laser which is determined by the incident laser intensity and the rubidium absorption coefficient. The factor of $1 / 2$ is due to the fact that only one-half of the absorbed photons end up polarizing the rubidium ground state. $\gamma_{x e}$ is the relaxation due to the buffer gas which is primarily lost to the spinrotation interaction, and $\gamma_{\text {wall }}$ is the wall relaxation rate. A typical pumping time is of the order of $0.1 \mathrm{msec}$. The xenon polarization increases much more slowly due to the small spin-exchange cross section. The polarization rate per xenon atom is given by:

$$
\frac{1}{T_{p u m p}}=[R b] \bar{\nu} \sigma_{e x}
$$

where $[\mathrm{Rb}]$ is the metal atom concentration, $\bar{\nu}$ is the average relative velocity between the xenon and the rubidium atoms, and $\sigma_{e x}$ is the spin-exchange cross section which is about $4 \times 10^{-21} \mathrm{~cm}^{2}$ [28]. The final xenon polarization, after long pumping times is given by [35]:

$$
P_{X e}=\frac{[R b] \bar{\nu} \sigma_{e x}}{[R b] \nu \sigma_{e x}+\gamma_{w a l l}} P_{R b}
$$

After the pumping light has been turned off, the rubidium polarization falls to that of the xenon spin polarization level within a spin-exchange time of about $[X e] \bar{\nu} \sigma_{e x}$ per rubidium atom which is about $1 \mathrm{msec}$. The rubidium polarization then follows the $z$ component of the xenon magnetization. Therefore, the rubidium allows for a sensitive optical detection mechanism that can be used to advantage in xenon NMR experiments. 


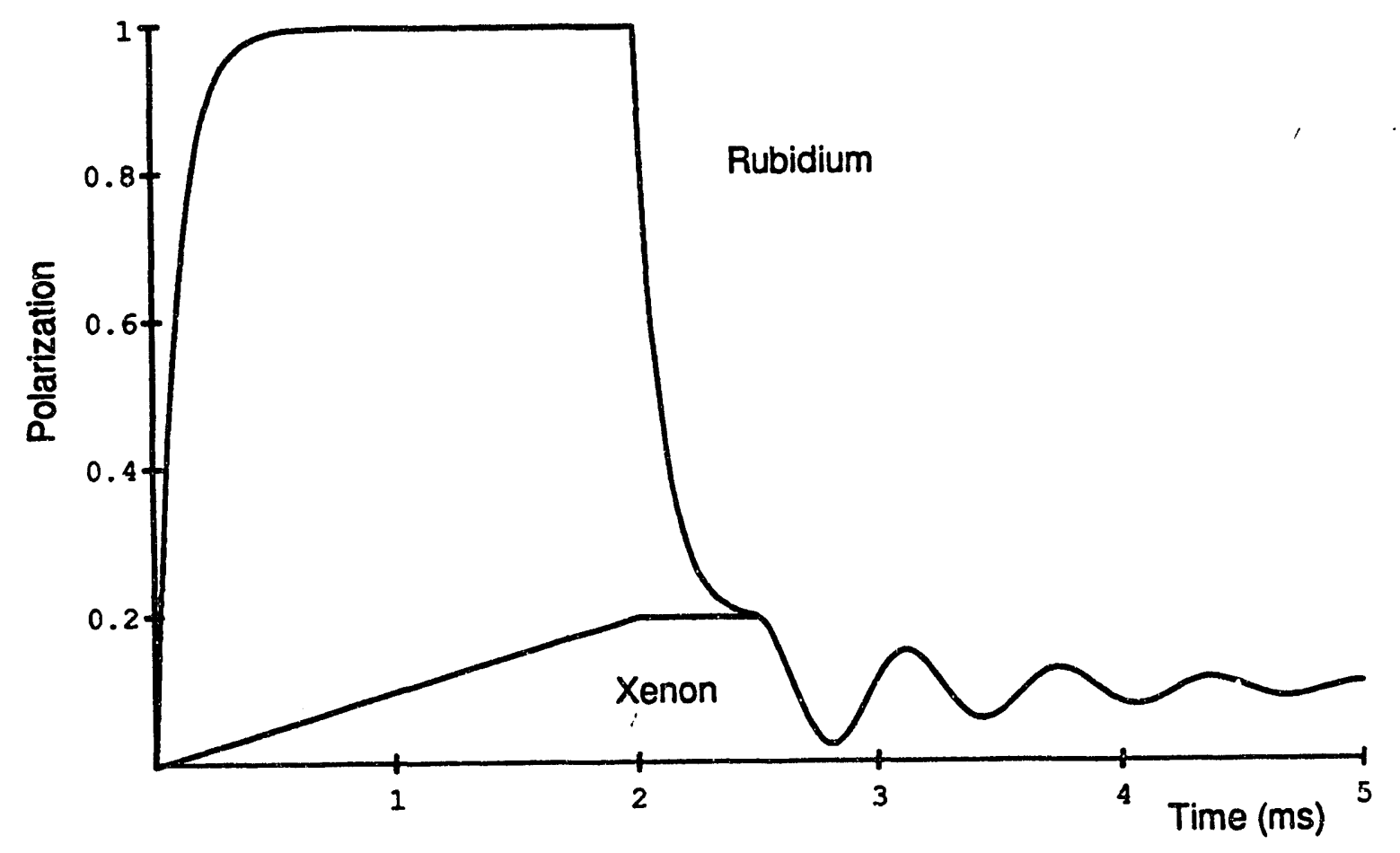

Figure 1.8: Optical pumping cycle with the pumping occurring during a time $t$, followed by optical detection of the rubidium polarization which follows the $z$ component of the xenon magnetization. The xenon pumping rate is exaggerated. 


\section{Chapter 2}

\section{NMR Studies of Xenon}

A short review of the xenon NMR literature is presented in this chapter which focuses on studies of xenon itself in the gas, liquid and solid phases. Experiments are described that concern the elucidation of xenon's interactions with itself using the accessible parameters of chemical shift, relaxation times and quadrupolar splittings (in the case of ${ }^{131} \mathrm{Xe}$ ) as discussed in Chapter 1.

\subsection{Gas Phase Experiments}

\subsubsection{Chemical Shifts}

After the first observations of xenon by NMR [36], gas phase studies concentrated on relaxation and chemical shift measurements as functions of the pressure [10] and temperature [37]. Jameson et al. [11] expressed the ${ }^{129} \mathrm{Xe}$ chemical shift as a virial expansion of the xenon pressure to describe the nonlinear behavior:

$$
\sigma=\sigma_{0}+\sigma_{1} \rho+\sigma_{2} \rho^{2}+\ldots
$$

Their results give a value of $0.548 \mathrm{ppm}$ chemical shift per amagat (density of xenon at STP, or $2 \times 10^{19}$ atoms $/ \mathrm{cc}$ ) for the linear term. Higher order terms, due to three 
(and more) body interactions that become important for densities above 50 amagats. These higher order xenon chemical shift terms are much more pronounced compared to those of fluorine or hydrogen because of xenon's much larger electron cloud polarizability. In other gases the effect of three-body interactions may safely be ignored in most situations. A theoretical expression for the chemical shift was calculated by Adrian [38] who showed that, during the lifetime of a collision of two xenon atoms, short-range electron exchange forces were responsible for the large density-dependent paramagnetic resonance shift observed. These calculations depend on the knowledge of the radial dependence of the chemical shift. Jameson [39] has produced the latest work on the chemical shift radial function by inverting the xenon chemical shift data using an the integral equation for the chemical shift:

$$
\sigma_{1}(T)=\int_{0}^{\infty} \sigma(r) e^{-V(r) / k T} r^{2} d r
$$

where $V(r)$ is the Xe-Xe pair potential [40]. Jameson showed that $\sigma_{1}$ was not a monotonically decreasing function of the radius as had been previously assumed in Adrian's work. In fact, the chemical shift function has a form similar to $V^{2}(r)$.

\subsubsection{Temperature Dependences}

The Jamesons are responsible for most of the work on the temperature dependence of the ${ }^{129} \mathrm{Xe}$ chemical shift. They found that the xenon chemical shift has a very large temperature dependence, ranging from $0.678 \mathrm{ppm} /$ amagat at $240 \mathrm{~K}$ down to $0.472 \mathrm{ppm} /$ amagat at $440 \mathrm{~K}$ which they fit by adding a temperature dependence to their virial expansion coefficients for the xenon chemical shift [37]. Their results are more accurate and differ considerably from earlier temperature measurements by Carr and coworkers [41]. Xenon apparently has a very nonlinear temperature dependence that is much larger than for both ${ }^{1} \mathrm{H}$ and ${ }^{19} \mathrm{~F}$ resonance shifts. Earlier, Adrian 
had calculated the temperature dependence of the xenon chemical shift using a pair distribution function and his expression for chemical shift for two interacting xenon atoms [42]. His results show the rather large temperature dependence evidenced by experiments plus the interesting effect of a maximum in the $\sigma_{1}(T)$ function at a temperature around $540 \mathrm{~K}$. To date, this has not been verified experimentally. However, see below for the xenon in NO gas results.

\subsubsection{Relaxation Studies}

A number of measurements have been made of the xenon relaxation times in the gas phase. Hunt and Carr showed that the relaxation time of ${ }^{129} \mathrm{Xe}$ is always inversely proportional to the pressure, with $T_{1}=2 \times 10^{5}$ sec-amagat [43], indicating that the correlation times are short (the "motional narrowing" regime). Also, paramagnetic impurities, such as oxygen, have a profound effect on $T_{1}$. Torrey [44] showed that Hunt and Carr's relaxation data were consistent with the spin-rotation interaction where the nuclear spin couples to the angular momentum of the short-lived molecular complex, allowing the xenon to relax much faster than would be expected if dipoledipole interactions were the domirant relaxation mechanism. Later work by Shizgal [45], using a Lennard-Jones $(6,12)$ potential, improved on the work by Adrian and obtained a ${ }^{129} \mathrm{Xe}$ relaxation time within $40 \%$ of the experimental result. The spinrotation interaction is also important in rubidium spin relaxation in optical pumping studies [28] and in the relaxation of spherical top molecules such as $\mathrm{CF}_{4}$ and $\mathrm{SF}_{6}$ [46] (although in the spherical tops the relaxation is due to an intramolecular coupling).

The relaxation of ${ }^{131} \mathrm{Xe}$ is dominated by collision-induced nuclear quadrupole interactions that couple the nuclear spin with rotational angular momenta of the colliding pair, as calculated by Adrian [47]. Adrian showed that exchange forces were again important in relaxation as they had been in explaining the large xenon 
chemical shift. His calculations are in agreement with measurements by Brinkmann

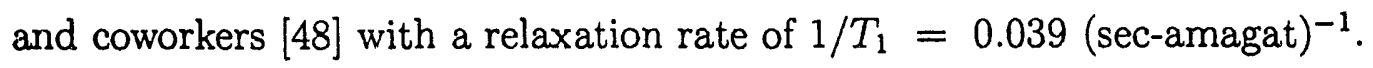

\subsection{Xenon with Other Gases}

The Jamesons and coworkers have made numerous investigations of mixtures of xenon with other gases in the hopes of elucidating the interatomic potential surface of xenon interacting with other atoms or molecules. These studies include xenon mixtures with: other rare gases [49] ( $\mathrm{Kr}$ and $\mathrm{Ar})$; spherical tops [50] $\left(\mathrm{CH}_{4}, \mathrm{CF}_{4}\right.$ and $\left.\mathrm{SiF}_{4}\right)$; nonspherical molecules [51] $\left(\mathrm{HBr}, \mathrm{HCl}, \mathrm{CO}_{2}, \mathrm{NO}_{2}, \mathrm{C}_{2} \mathrm{H}_{2}, \mathrm{C}_{2} \mathrm{H}_{4}\right.$ and $\left.\mathrm{BF}_{3}\right)$; linear molecules [52] $\left(\mathrm{CO}\right.$ and $\left.\mathrm{N}_{2}\right)$; and paramagnetic species [53] $\left(\mathrm{O}_{2}\right.$, and $\left.\mathrm{NO}\right)$. From their earlier work on pressure and temperature dependent virial expansions of the xenon chemical shift in pure xenon gas, the Jamesons could examine xenon in other gas mixtures by simply subtracting the xenon-xenon contribution. For the spherical top molecules, the results are as expected: the temperature dependence is of the same form as the xenon-xenon virial coefficients except that they are smaller for the spherical top molecules probably due to their smaller polarizabilities. An attempt to normalize the $\sigma_{1}(r)$ functions in terms of the equilibrium collision distance and well depth works well in for xenon in mixtures with similar partners, such as the other rare gases, or the spherical tops. However, between groups, the differences are substantial so that this is not possible to generalize the method [50].

The linear molecules studied behave much differently. As shown in Figure 2.1, the second virial coefficient for $\mathrm{CO}$ and $\mathrm{N}_{2}$ is an increasing function of the temperature unlike all of the other (diamagnetic) buffer gases studied. According to the authors, this fact is not an indication that the functional form of $\sigma_{1}(r)$ is radically different for linear molecules, but simply that the average collision radius for xenon with $\mathrm{CO}$ 
and $\mathrm{N}_{2}$ is much different (smaller) than for the other collision partners studied.

The xenon chemical shifts are also quite different in the presence of paramagnetic buffer gases. Calculations of xenon in paramagnetic gas mixtures were made in 1972 by Buckingham and Kollman [54]. They considered the xenon-NO and xenon- $\mathrm{O}_{2}$ collision pairs and determined that the Fermi-contact interaction was responsible in both cases for the unusually large resonance shifts observed in mixtures of these gases. Just as in the case of $\mathrm{Rb}-\mathrm{Xe}[27]$, the valence electrons of the paramagnetic species have a large spin density at the xenon nucleus due to the enhancement by the Pauli exclusion principle.

The Jamesons and coworkers have determined the density-dependent chemical shifts of ${ }^{129} \mathrm{Xe}$ due to the presence of $\mathrm{O}_{2}$ and NO, finding that $\sigma_{1}=-1.25 \mathrm{ppm} /$ amagat for oxygen and $-0.924 \mathrm{ppm} /$ amagat for NO buffer gases. These are much larger (by a factor of 6-10) than for argon, which is of the same size and polarizability as the two paramagnetic species. Their results on temperature dependent resonance shifts [55] support the proposition of a Fermi contact induced shift. Further work by these authors established that NO had a similar effect on the ${ }^{129} \mathrm{Xe}$ resonance frequency, although not quite as large in magnitude. The contact term, given by:

$$
\sigma_{1}=\frac{-4 \pi \text { overline } \mu_{e f f}^{2} 9 k T}{\int} \rho_{\text {spin }} e^{-V(r) / k T} d \tau
$$

is proportional to the electron spin density of the paramagnetic species found at the xenon nucleus, averaged over the collision pair configurations, and is larger for NO than $\mathrm{O}_{2}$. However, oxygen's two unpaired electrons make $\sigma_{1}$ larger overall for $\mathrm{O}_{2}$.

\subsection{Solid and Liquid Xenon}

Norberg and coworkers [56] investigated ${ }^{129} \mathrm{Xe}$ chemical shifts, relaxation times and self-diffusion coefficients in liquid and solid xenon. Below the melting point, the 


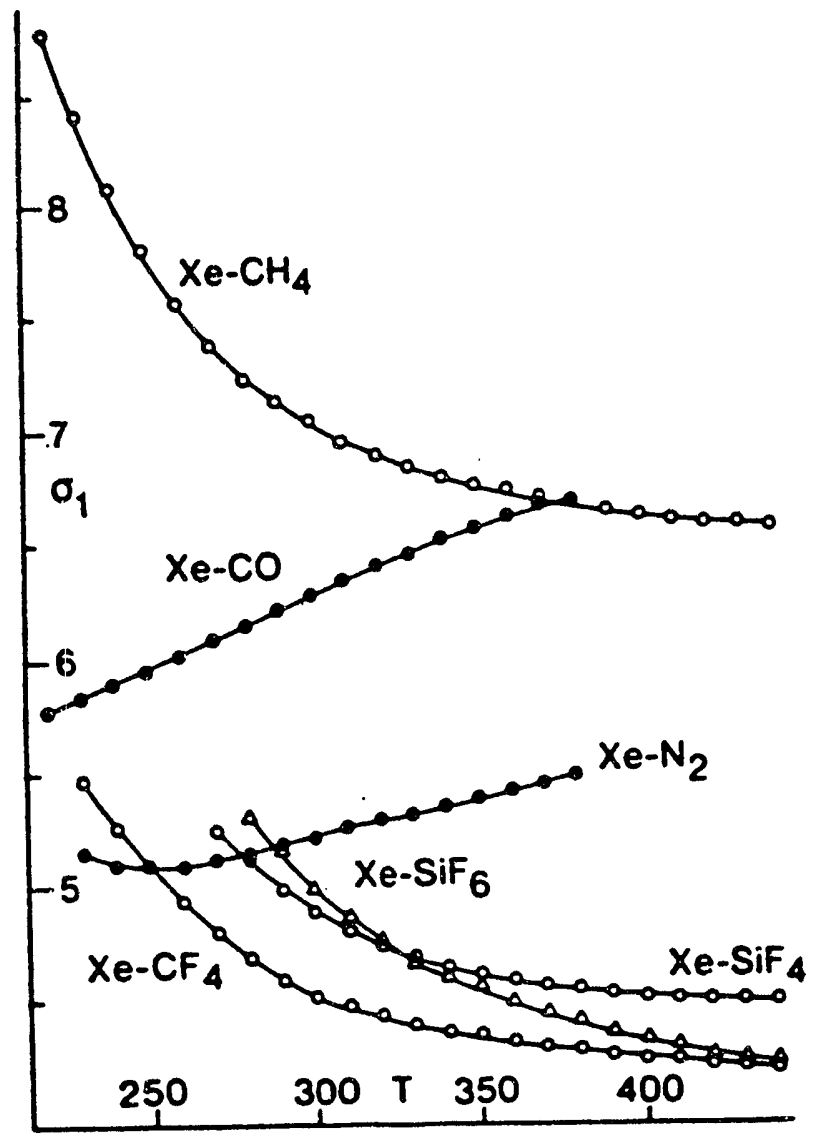

Figure 2.1: Temperature dependence of the ${ }^{129} \mathrm{Xe}$ second virial coefficient in xenon gas and other gases. Adapted from Jameson et al. [52]. 
xenon line width increases monotonically until about $118 \mathrm{~K}$ where the rigid lattice line width of $300 \mathrm{~Hz}$ is reached. The observed line width is in agreement with the Van Vleck formulation for a dipolar-coupled solid. A large discrepancy occurred in the measured slopes of chemical shifts versus density for solid xenon. Brinkmann and Carr reported slopes of $6.0 \mathrm{ppm} /$ amagat [57] whereas Norberg and coworkers measured $18.2 \mathrm{ppm} /$ amagat $[56,58]$. Cowgill and Norberg [59] later reproduced the Carr data, which had been in agreement with theoretical work [60].

Warren and Norberg [58] studied relaxation in ${ }^{131} \mathrm{Xe}$ solid. The measured relaxation times for solid ${ }^{131} \mathrm{Xe}$ were in qualitative agreement with a quadrupolar relaxation caused by a two-photon mediated Raman process [61] for solids with a Debye distribution of phonon energies. The Debye temperature for solid xenon is estimated to be $55 \mathrm{~K}$. Single phonon relaxation processes are limited by the numbers of phonons at $\omega$ or $2 \omega$, whereas a two phonon Raman process couples the nuclear quadrupole spin to pairs of phonons with energies $\gamma_{1}-\gamma_{2}=\omega$ or $2 \omega$ and thus involves all of the phonons. Van der Waals and exchange forces are responsible for creating the fluctuating electric field gradients which cause relaxation. At temperatures near the melting point, the $T_{1}$ data deviates from the theory, a fact which the authors ascribe to quadrupolar relaxation caused by diffusion to paramagnetic impurity centers. Warren and Norberg [62] also studied both ${ }^{129} \mathrm{Xe}$ and ${ }^{131} \mathrm{Xe}$ dipolar and quadrupolar echos at $4.2 \mathrm{~K}$. They found that the $\mathrm{T}_{2}$ of solid ${ }^{131} \mathrm{Xe}$ is as short as $8 \mathrm{msec}$ at such cold temperatures.

Carr and coworkers have used xenon NMR to examine the xenon liquid-vapor coexistence curve near the critical temperature $T_{c}$ [63]. In a limited range near the critical point, a single power law $B e^{\beta}$ describes the data with $\beta=0.305$. However, the data are better described by including a correction term in agreement with results of other phase phenomena. 


\subsection{Xenon Thin Films and Clusters}

\subsubsection{Xenon on Graphite}

The xenon-graphite system has been the focus of many studies including adsorption [64], neutron and X-ray scattering [65], and $\operatorname{NMR}[66,67]$ due to the importance of surface interactions with atomic monolayers and the possibility of observing the structure and dynamics of reduced dimensional phenomena [68]. NMR is not capable of matching the sensitivity of the scattering experiments, and therefore the two NMR studies to date have made use of high surface area graphite material (Graphoil [69]), where the basal planes have been expanded by exfoliation with ferric chloride at high temperatures. A continuous wave (CW) NMR experiment [66] indicated the possibility of observing two-dimensional phase transitions via chemical shift and line width changes in the ${ }^{129} \mathrm{Xe}$ NMR spectra. The observed line width of the multilayer spectrum is too large to be due solely from bulk dipole-dipole interaction considerations, a fact the author attributes to multiple adsorption sites and the large diamagnetic susceptibility of graphite. A second spectrum is indicative of the large xenon-graphite interaction, with a chemical shift of over $325 \mathrm{ppm}$, well beyond the bulk solid value. The line width may be due to a hexatic (planar hexagonal), commensurate phase which was recently observed by Birgineau et al. in X-ray experiments [65]. Recently, Neue [67] observed asymmetric line shapes of 1 to 3 monolayer coverages of xenon on Graphon (graphitized carbon) at temperatures between $95 \mathrm{~K}$ and $140 \mathrm{~K}$. The line shapes are again indicative of the large magnetic susceptibility of the graphite substrate. At $158 \mathrm{~K}$ Neue observed a small splitting of $180 \mathrm{~Hz}$ which he ascrib nd to the hexatic phase. Neue also observed two minima in relaxation time measurements of ${ }^{129} \mathrm{Xe}$. At $160 \mathrm{~K}$, the relaxation is due to rapid motion of xenon. At lower temperatures, the relaxation is probably due to the diffusion of a mobile fraction of xenon 
to paramagnetic impurity sites.

\subsubsection{Frozen Xenon Thin Films}

As will be discussed in Chapter 8, optical pumping methods can be applied to NMR of xenon and can be used to enhance the signal from xenon thin films [70]. The effects of the anisotropic diamagnetic susceptibility are apparent in the distributions of the $1 \mu \mathrm{m}$ films. The line shape for a model film in a cylindrical geometry is shown in Figure 2.2, (and the experimental data later, in Figure 8.2(a)). The o:erall magnitude of the diamagnetic susceptibility is $14.8 \mathrm{ppm}$ in accordance with the reported bulk value [71].

\subsubsection{Clusters of Xenon in Na-A Zeolite}

Conventional xenon NMR has also been used to study the distributions of finite clusters of xenon [72]. Xenon may be packed inside the small, independent supercages of $\mathrm{Na}-\mathrm{A}$ zeolite under conditions of elevated temperature and pressure that allow xenon to pass through the small $\alpha$-cage windows. Upon rapid cooling, the xenon population distributions are frozen-in, allowing one to probe the high temperature distributions with NMR at room temperature. The ${ }^{129} \mathrm{Xe}$ chemical shift is sensitive to xenon's local interactions and can be used to distinguish different populations of xenon among the $\alpha$-cages of $\mathrm{Na}-\mathrm{A}$ zeolite [73]. In Figure 2.3, a spectrum for a xenon in Na-A zeolite sample loaded at 8 atm xenon pressure and $250{ }^{\circ} \mathrm{C}$ demonstrates the distinct chemical shifts for cage occupancies from 1 to 5 xenons atoms. After normalizing the integrated intensities of the peaks by dividing by the cage occupancies [74], ${ }^{129} \mathrm{Xe}$ NMR reveals that the distribution of xenon occupancies among the cages follows a binomial distribution at low loadings. 

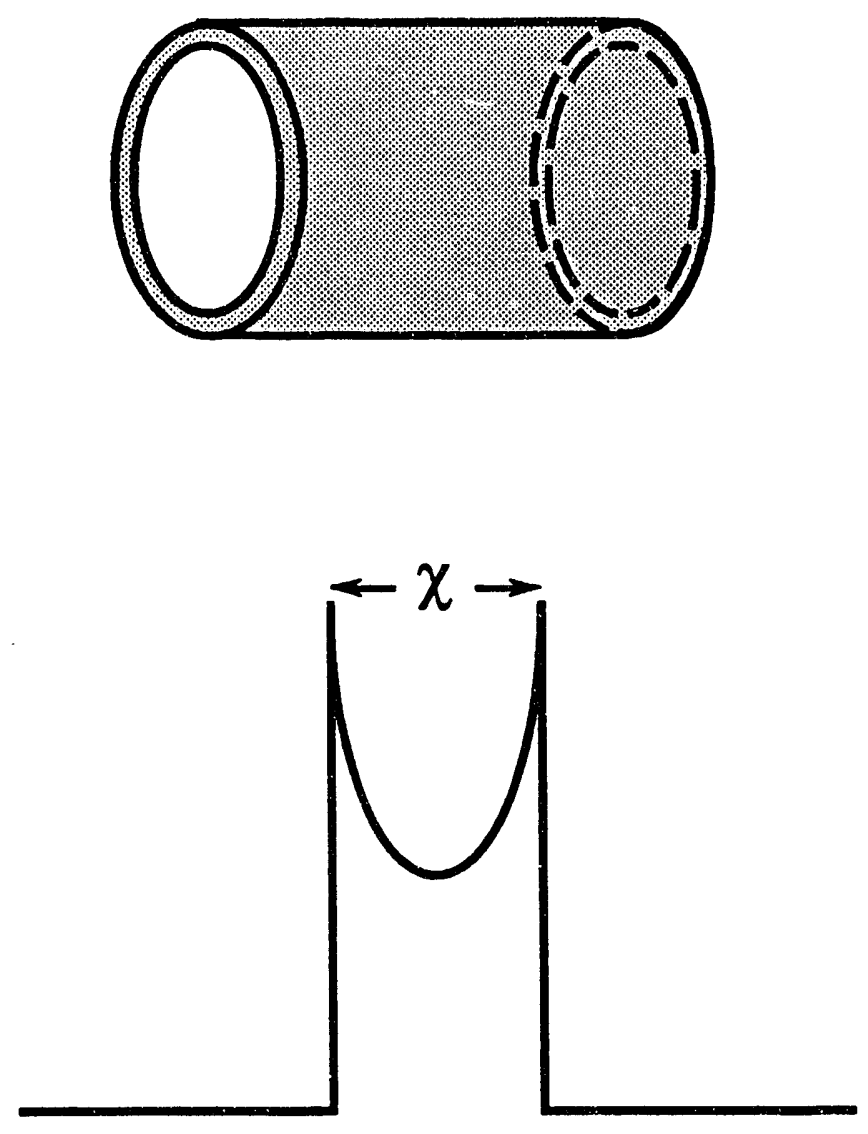

Figure 2.2: NMR of a thin film of xenon enhanced by optical pumping. The line shape of the solid is affected by the bulk diamagnetic susceptibility of xenon and the geometry of the film. (a) Schematic diagram of a xenon thin film inside a cylindrical sample cell. (b) The NMR spectrum has a 2D powder pattern line shape. 


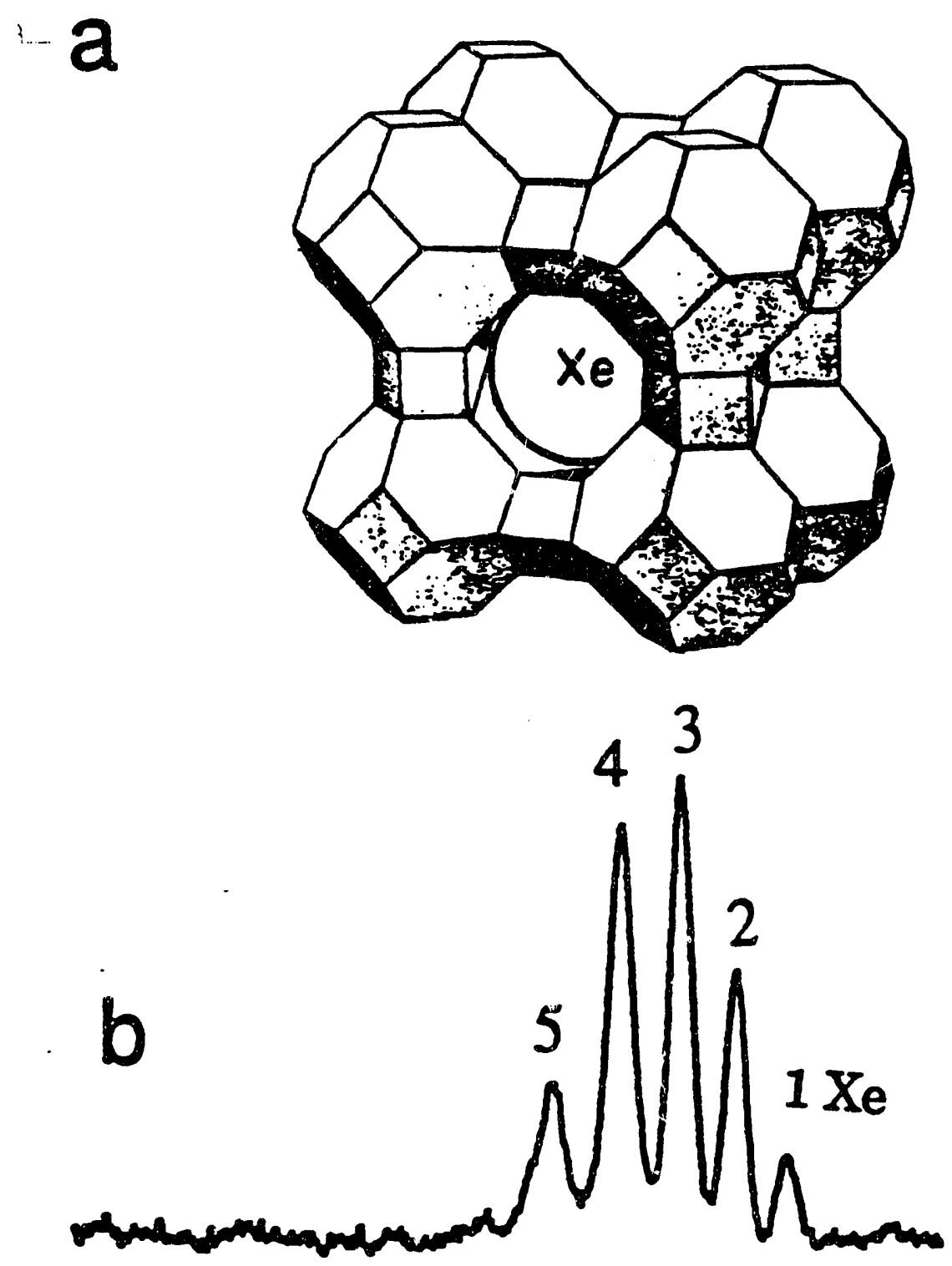

Figure 2.3: (a) Schematic diagram of a xenon atom inside the micro pores of zeolite. (b) Room temperature ${ }^{129} \mathrm{Xe}$ NMR spectra for xenon occluded in Na-A zeolite at $523 \mathrm{~K}$ and at $8 \mathrm{~atm}$. The discrete, chemically-shifted peaks correspond to $\alpha$-cages containing different numbers of occluded xenon atoms as indicated by the numbers above the peaks. 
At higher loadings, the finite atomic volume of the xenon atoms must be taken into account, and a hypergeometric distribution [75] well describes the xenon occupancies as suggested by Güémez and Velasco [76]. As will be shown in Chapter 5 , at xenon loadings above about three xenons per $\alpha$-cage, the occupancies are described by the hypergeometric distribution. At the highest loadings, with an average occupancy $\bar{n} \circ 5.9$, the experimental data are more narrow than either the binomial or hypergeometric distributions. This may be due to the presence of motion of the xenon that would provide a less-accommodating configuration inside the $\alpha$-cage. These results are in agreement with earlier Monte-Carlo simulations [77].

\subsection{Optically-Pumped Xenon Experiments}

As described in Chapter 1, highly polarized xenon gas may be obtained through spin exchange collisions with optically-pumped rubidium vapor. Happer has investigated the spin exchange mechanism in both ${ }^{129} \mathrm{Xe}$ and ${ }^{131} \mathrm{Xe}$ finding that ${ }^{129} \mathrm{Xe}$ polarizes readily with a binary cross section of $2 \times 10^{-21} \mathrm{~cm}^{2}$, and depends on the rubidium density and polarization [79]. Happer determined that spin exchange efficiency was about $5 \%$, given approximately by the square of the ratio of spin exchange interaction to the spin-rotation interaction. This ratio is about 4 , so most of the polarized rubidium is relaxed by spin rotation. At high densities, the rubidium polarization can also be reduced by radiation trapping, where de-excited rubidium atoms emit photons with the wrong polarization that are subsequently absorbed by other rubidium atoms, causing depolarization [80]. However, the addition of nitrogen o: helium gas quenches this mechanism and large polarizations of xenon ( $30 \%)$ at xenon pressures of about 1 atm have been produced using high-power ( 5 watt) lasers [78]. 
The effect of polarized rubidium vapor on the xenon resonance can be seen as a frequency shift [81] of some tens of millihertz in low magnetic fields using optical detection methods. This is similar to the Knight shift [82] observed in metals due to the interaction of polarized electrons with the nuclear spin, and is linearly dependent on the polarization of the electrons (and thus the temperature for high field NMR experiments), This result can be verified by varying the light source intensity or by reversing its polarization.

The primary relaxation mechanism for optically pumped ${ }^{129} \mathrm{Xe}$ in the presence of rubidium vapor is again the Fermi contact interaction that acts in the reverse direction when the polarized light source is turned off. The rubidium polarization quickly drops to the level of the xenon polarization, and can be detected optically $[83,84]$. Of course, at low rubidium densities this effect is diminished and collisions with the walls of the container become important at xenon densities below about 20 amagats. Cell wall coatings such as Surfrasil [85], or long-chain hydrocarbons are found to reduce the wall-induced relaxation rate to about 2 per hour probably because the coatings cover up paramagnetic centers.

\section{6 ${ }^{131}$ Xe Optical Pumping Experiments}

Optical pumping experiments have also been applied to the study of ${ }^{131} \mathrm{Xe}$, although the reduced spin-exchange efficiency and relaxation time due to quadrupolar interactions are sometimes a limiting factor. Volk and coworkers [86] were the first to observe the effects of collisions of ${ }^{131} \mathrm{Xe}$ with the walls of their non-spherical sample cells that produced a beat pattern in the decay of the transverse ${ }^{131} \mathrm{Xe}$ polarization. Although they were not able to discern the very small quadrupolar splittings due to resolution limitations, they ascribed their obscrvations to a quadrupolar dephasing 
mechanism which had previously been seen in ${ }^{201} \mathrm{Hg}$ (nuclear spin $3 / 2$ also) optical pumping experiments by Cohen-Tannoudji $[31,87]$. The dephasing is due to the random fluctuations of the electric field gradient experienced by the nucleus at the surface of the cell. The dephasing rate is written:

$$
A(\theta)=\frac{\tau_{s}}{\tau_{c}+\tau_{s}} \frac{e^{2} Q q}{4}\left(3 \cos ^{2} \theta-1\right)
$$

where $\tau_{s}$, is the sticking time, $\tau_{c}$ is the electric field gradient correlation time, and $\theta$ is the angle between the quadrupolar interaction principle axis frame and the laboratory or rotating frame (which is relevant for nutation experiments). The sticking time may be expressed as:

$$
\tau_{s}=\tau_{\circ} e^{-E_{a} / k T}
$$

where $E_{a}$ is a surface activation energy. A typical value of $E_{a}$ for bare Pyrex cells is $-0.03 \mathrm{eV}$ or $-7 \mathrm{kcal} / \mathrm{mole}$.

In flat optical cells, Happer [84] has observed splittings in the NMR frequencies on the order of $100 \mathrm{mHz}$. The quadrupolar splitting scales with the asymmetry of the cell such that:

$$
\Delta \omega=\frac{1}{2} \bar{v}\langle\theta\rangle\left(\frac{1}{h}-\frac{1}{d}\right)
$$

where $\bar{v}$ is the average xenon velocity, $(1 / \mathrm{h}-1 / \mathrm{d})$ is the cell asymmetry, and $\langle\theta\rangle$ is the mean twist angle of the nuclear polarization occurring at the cell surface due to the quadrupolar interaction, averaging over the cell surface and sticking times. Happer's mean-twist angle is the product of the sticking time and the quadrupolar interaction strength and can be determined by plotting the quadrupolar splitting versus the cell asymmetry. The observed splittings have a slope $\bar{v}\langle\theta\rangle / 4 \pi$ equal to $100 \mathrm{mHz}-\mathrm{cm}$. Using a mean speed of overlinev $=2.4 \times 10^{4} \mathrm{~cm} / \mathrm{sec}$ at $82^{\circ} \mathrm{C}$, the mean-twist angle is $38 \times 10^{-6}$ radians. 


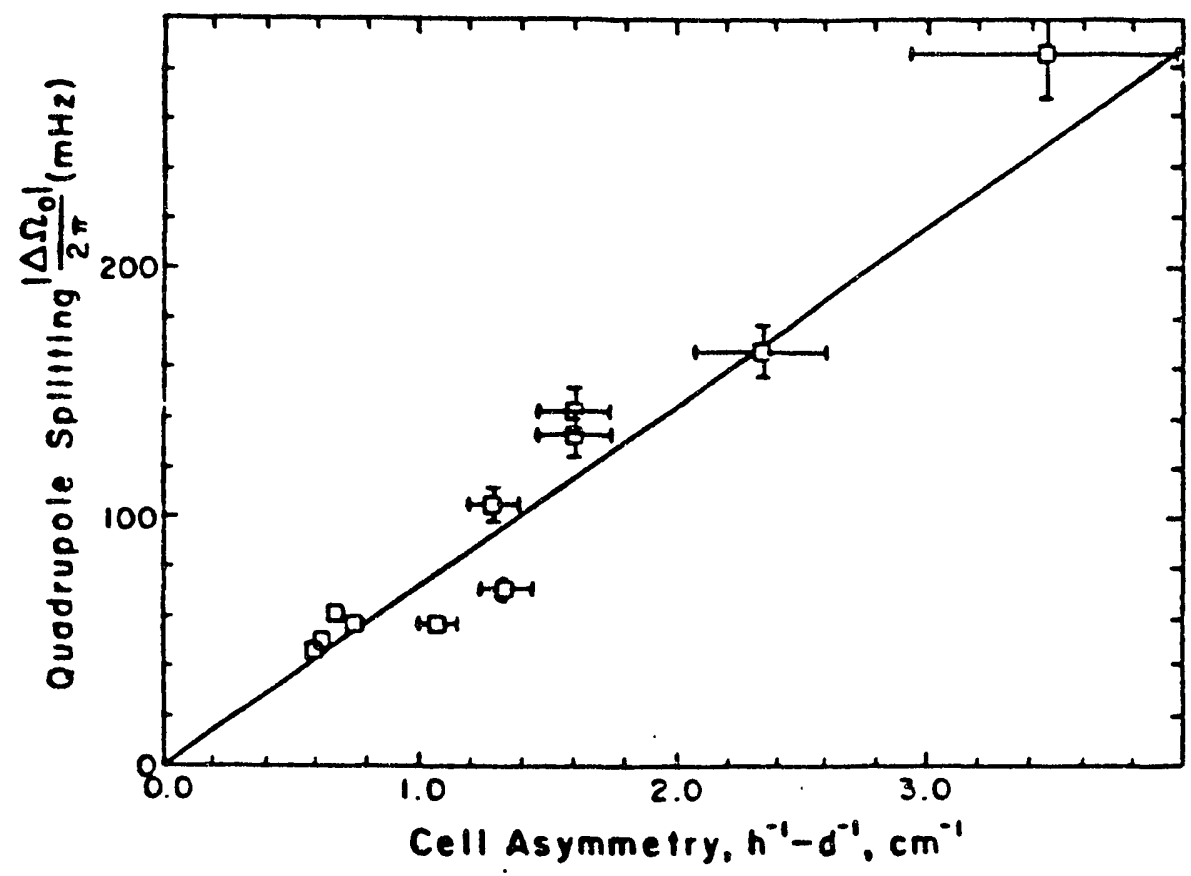

Figure 2.4: Quadrupolar splittings versus the cell asymmetry in Pyrex cells, at 82 ${ }^{\circ} \mathrm{C}$. Adapted from Happer and coworkers [84]. 
Happer and coworkers have derived a more general expression [88] for the quadrupolar relaxation due to surface collisions by explicitly integrating over the sticking times. Their expression for the relaxation rate is:

$$
\gamma_{1}=\frac{\bar{v} S}{10 V}\left\langle\theta^{2}\right\rangle
$$

where $\left\langle\theta^{2}\right\rangle$ is the mean squared twist angle that acts as a random fluctuation to cause relaxation. Happer's results indicate that the magnitude of the instantaneous quadrupolar interaction felt by the xenon nucleus at a particular adsorption site is much larger than the ensemble average value.

Investigations of surface treatments indicate that, Surfrasil coatings, useful for increasing ${ }^{129} \mathrm{Xe}$ relaxation times, have the reverse effect on quadrupolar ${ }^{131} \mathrm{Xe}$ relaxation $[88,89]$ where little or no polarized ${ }^{131} \mathrm{Xe}$ is observed due to quadrupolar relaxation at the surface of Surfrasil-coated cells. However, the addition of $\mathrm{H}_{2}$ to the cells, has been found to increase the relaxation time for ${ }^{131} \mathrm{Xe}$ experiments [86] which is probably due to the formation of $\mathrm{Rb}-\mathrm{H}$ at the surface. In Figure 2.5, the nutation signal for ${ }^{131} \mathrm{Xe}$ in a cylindrical flat cell with height $3 \mathrm{~mm}$ and added hydrogen gas is shown, along with its Fourier transform. The quadrupolar splitting at $100^{\circ} \mathrm{C}$ is $340 \mathrm{mHz}$. It appears that hydrogen has the added (and unexplained) effect of increasing the quadrupolar splittings [89]. 

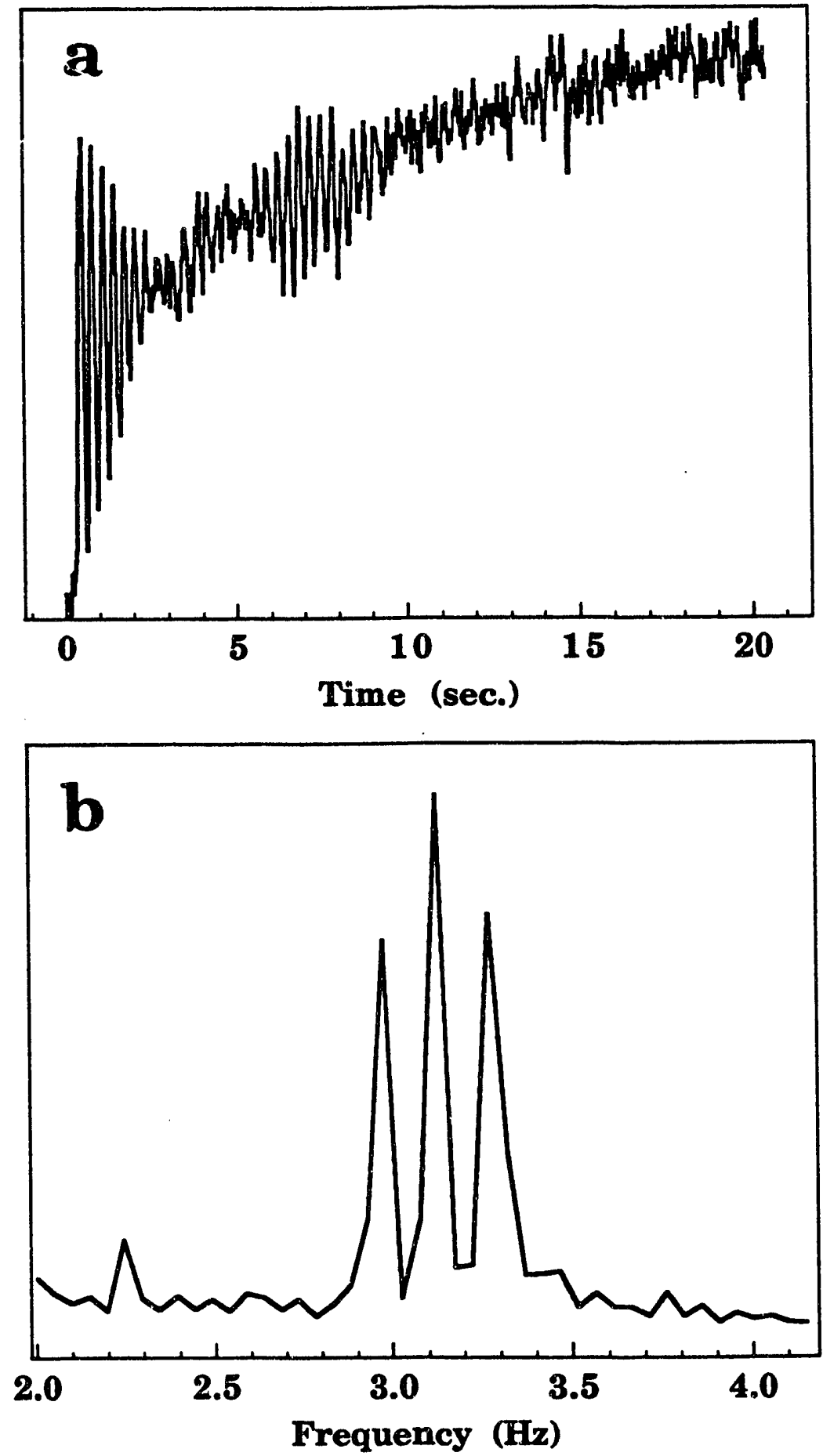

Figure 2.5: (a) ${ }^{131} \mathrm{Xe}$ nutation signal in a flat cylindrical cell with added hydrogen, $3 \mathrm{~mm}$ high and $50 \mathrm{~mm}$ in diameter at $100{ }^{\circ} \mathrm{C}$. The background decay is due to the ${ }^{129} \mathrm{Xe}$ background. (b) Fourier transform of (a). The quadrupolar splitting is 340 $\mathrm{mHz}$. 


\section{Chapter 3}

\section{Xenon as a Probe of Structure}

Much of the xenon NMR literature deals with the elucidation of the structure of different materials that interact with xenon. Such studies span a large range, including: proteins, powdered high surface area materials, catalysts, inclusion compounds and so forth. The goal of this chapter is to briefly cover some of the more interesting applications of xenon NMR and to point out other materials that will be interesting to study with xenon NMR in the future.

\subsection{Low Surface Area Studies}

Recent experiments on optical pumping of xenon [5] have extended the study by xenon NMR to materials with surface areas in the range of $1-10 \mathrm{~m}^{2} / \mathrm{g}$. As will be discussed in Chapter 7 in detail, xenon that is optically pumped in the fringe field below the NMR magnet may be shuttled to high field and adsorbed onto a variety of powdered materials. The enhancement over Boltzmann polarization is over three orders of magnitude. Conventional NMR detection of the highly polarized xenon yields information about the xenon-surface and xenon-xenon interactions. A full study of polyacrylic acid was undertaken and showed that the xenon chemical shift 
data had large temperature and coverage dependencies, which could be understood in terms of a large xenon-surface interaction with the polymer and xenon's diffusion over the surface. Using a model that takes into account the probability of finding the xenon at the surface, the surface diffusion constant obtained was $2 \times 10^{-5} \mathrm{~cm}^{2} / \mathrm{sec}$ at $130 \mathrm{~K}$ and coverage of one monolayer.

This new method of detecting optically-pumped xenon in high magnetic field promises to extend the xenon NMR work to a large variety of materials without the constraint of high surface area or especially strong adsorption properties. Other materials presently under study include: low surface-area natural graphite, molecular crystals such as benzanthracene, and alkali halide crystals. An exciting possibility in the near future will be to use cross-polarization to selectively enhance the surface nuclei of the material of interest.

\subsection{Zeolites}

Probably the most widespread application of xenon NMR is to the study of zeolites which find their primary importance in catalysis. The microporous nature of zeolites, their advantageous adsorption properties, and their availability in a variety of structures and geometries make them useful in many parts of the chemical industry [90]. Schematic drawings of some of the more useful zeolites, Na-Y, Na-A, ZSM-5, are

shown in Figure 3.1. Naturally occurring or synthetic [91] zeolites find their use in petroleum cracking, hydrogenolysis reactions and gas separations. The popular Na-Y zeolite has a unit cell chemical formula: $\mathrm{Na}_{x}\left(\mathrm{AlO}_{2}\right)_{x}\left(\mathrm{SiO}_{2}\right)_{192-x},($ with $\mathrm{x}=50$ to 80), and contains 32 exchangeable cations, 4 for each of the $813 \AA$ diameter supercages in the unit cell. The zeolite chemistry may easily be changed to suit the needs of the chemist. Xenon NMR has been used to elucidate zeolite structure [2], examine the 
2

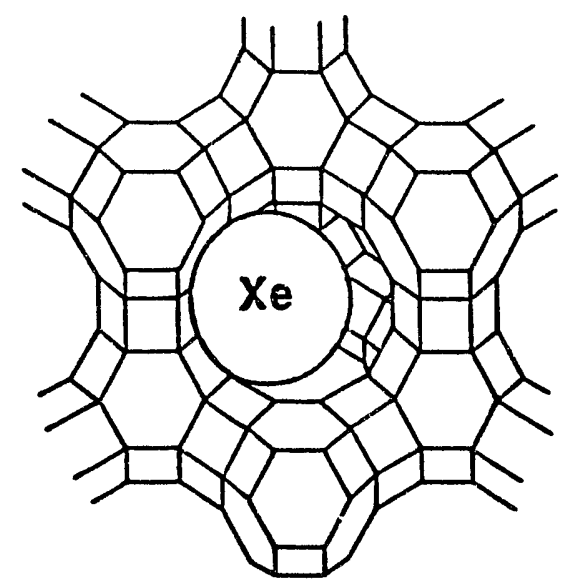

b

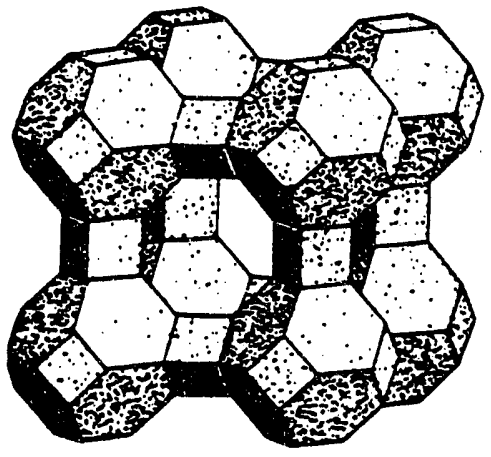

c

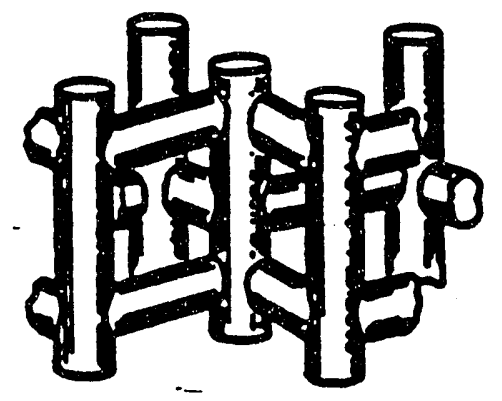

Figure 3.1: Schematic diagram of different zeolite structures. (a) Na-Y, (b) Na-A and (c) ZSM-5. Na-Y and Na-A zeolites have a supercage structure while ZSM-5 consists of interconnecting channels. 
effects of cation substitution and other chemical modifications to the zeolite support, monitor a variety of heat treatments, characterize their catalytic properties, and probe guest-host interactions. Several recent reviews have appeared that document much of the current literature on xenon NMR of zeolites [92, 4]. However, despite the numerous studies, ${ }^{129} \mathrm{Xe}$ NMR, in the (paraphrased) words of Cecil Dybowski ${ }^{1}$ "has not proved to be the powerful analytical tool for zeolite studies as was once hoped." This is due to the limitation of a single parameter (the chemical shift) to describe phenomena, plus the fact that the shift has a complicated dependence on temperature, pressure and the material under study. Typically, a lot of data must be acquired in order to understand the various phenomena, while the results remain empirical in nature. Nevertheless, when combined with other techniques such as X-ray crystallography or IR spectroscopy, xenon NMR can give valuable information such as structure determination, or can be used to follow changes in acidity, adsorption or catalytic sites and the like.

\subsubsection{Pure Zeolites: Na-Y, Na-A ZSM-5 and Others}

The first and probably still the largest area of ${ }^{129} \mathrm{Xe}$ NMR research is the study of zeolite structure in its unaltered form. Fraissard and coworkers first indicated xenon's usefulness in the study of different structural aspects, pore sizes and cation influences of several zeolites in a series of papers starting in $1982[2,93,94]$. He determined that xenon chemical shifts for many different zeolites with different chemical formulae all had a linear dependence on xenon concentration (at room temperature) in numbers of xenons per zeolite supercage except for cases of zeolites with highly-exchanged $2+$ cations such as $\mathrm{Ca}-\mathrm{Y}$ zeolite [2]. In Figure 3.2 , the ${ }^{129} \mathrm{Xe}$ chemical shift for

\footnotetext{
${ }^{1}$ Rocky Mountain Conference, Denver, CO (1991).
} 
several zeolites with different chemical forms is shown versus the xenon concentration. All data are linear except for the $\mathrm{Ca}-\mathrm{Y}$ zeolite where the pronounced effect of the exchanged cations can readily be seen. Fraissard extended the xenon gas phases work of Jameson et al. $[11,37]$ (see Chapter 1) by writing the chemical shift of ${ }^{129} \mathrm{Xe}$ in zeolites in terms of a xenon-surface interaction, a pressure dependent term plus other terms. Fraissard's equation is written:

$$
\delta_{X e}=\delta_{\circ}+\delta_{S}+\delta_{(X e-X e)} \rho+\delta_{E}
$$

where $\delta_{0}$ and $\delta_{X e-X_{e}}$ are the xenon reference and pressure dependent shifts as before, $\delta_{S}$ is the chemical shift due to the xenon-surface interaction, and $\delta_{E}$ is a term due to the electric field of a cation. In $\mathrm{Na}-\mathrm{Y}$ zeolite, for instance, the ${ }^{129} \mathrm{Xe}$ chemical shift is linear with the xenon concentration with an intercept of $58 \pm 2 \mathrm{ppm}[2,5]$ and a slope, $\delta_{X e-X e}$ of $0.40 \mathrm{ppm} /$ amagat, as compared to $0.548 \mathrm{ppm} /$ amagat as measured by Jameson et al. for xenon in the gas phase [11]. Recently, Cheung [95] has shown that the reduction in the slope is proportional to the adsorption energy of the zeolite framework.

The intercept of data such as shown in Figure 3.2 is $\delta_{S}$ which is the extrapolated to zero xenon pressure value of the chemical shift, and indicates the xenon-surface interaction strength since xenon-xenon collisions are no longer important. Fraissard and coworkers related the xenon-surface term to the mean free path between surface collisions of xenon in the zeolite structure, and showed a good correlation between $\mathrm{Na}-\mathrm{Y}$, rho, ferrierite and mordenite structures[96]. They determined an empirical formula for the xenon chemical shift due to the surface:

$$
\delta_{S}=\frac{2.054}{2.054+\ell}
$$

where $\ell$ is the mean free path of xenon between surface collisions. Another explanation is possible due to the curved surface of the zeolite cavity, and its effect of 


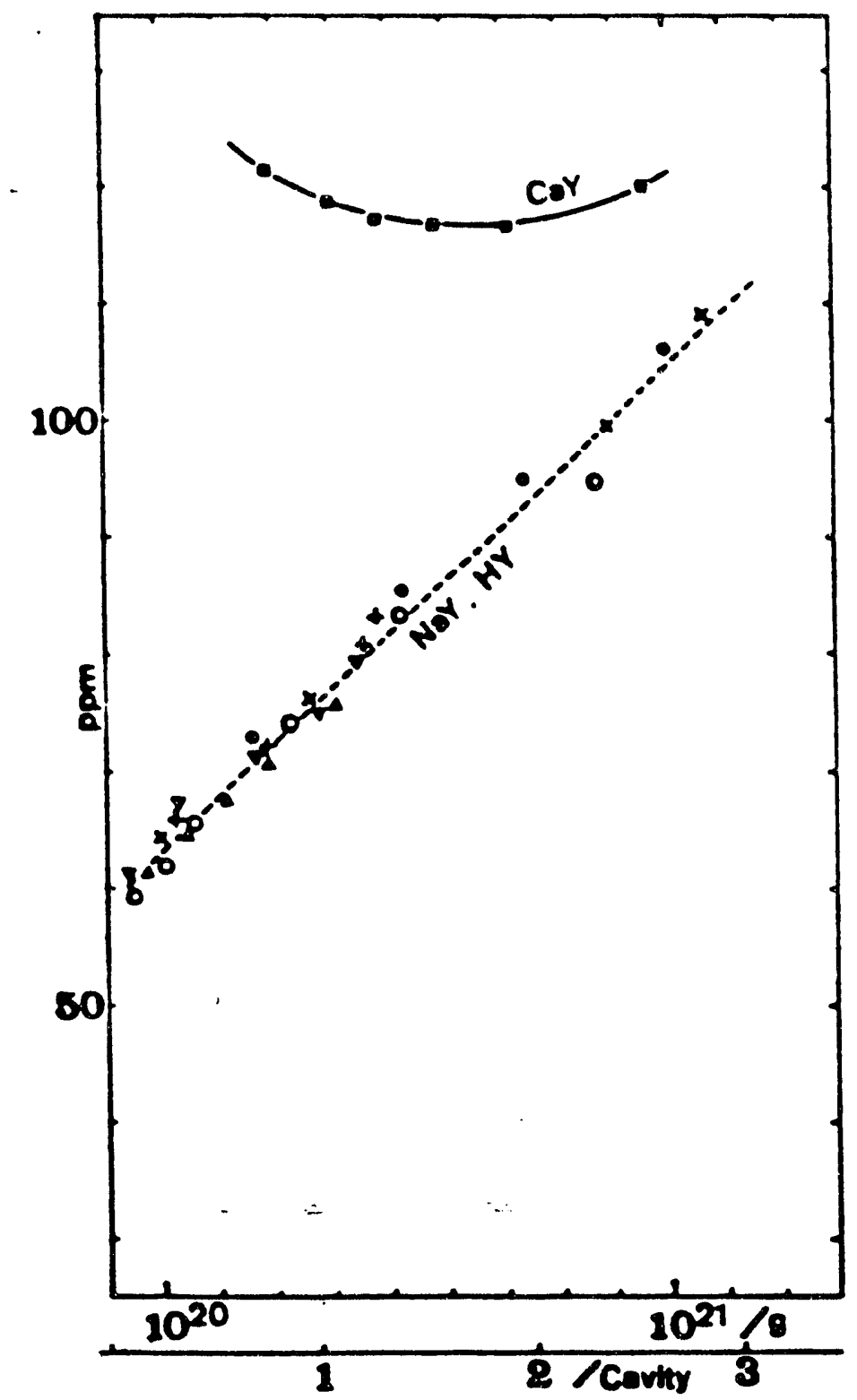

Figure 3.2: ${ }^{129} \mathrm{Xe}$ chemical shift for xenon adsorbed in $\mathrm{Na}-\mathrm{Y}$ (a) and $\mathrm{Ca}-\mathrm{Y}$ zeolites. Adapted from Fraissard and coworkers [2]. 
the adsorption properties that result in ine polarization of the xenon electron clouds and a paramagnetic shift of the xenon resonance (see Chapter 1). Derouane [97] determined a relationship between the chemical shift and the surface curvature:

$$
\delta=A(1-d / 2 a)^{-3}
$$

where $A$ is a molecular constant, $d$ is the distance from the xenon nucleus to the wall, and $a$ is the pore radius. Void spaces in many other zeolite-like materials have been investigated in terms of their xenon-surface interactions, crystallinity [98] and chemical composition $[2,99]$.

Some interesting effects due to the geometry of zeolite pore spaces have also been observed. Springer-Huet anc oworkers [100] reported that the ellipsoid-shaped long channels in SAPO-11 and $\mathrm{AlPO}_{4}-11$ caused a chemical shift anisotropy (CSA) that could be seen in the ${ }^{129} \mathrm{Xe}$ line shape (see Figure 3.3). As the amount of adsorbed xenon was increased, the sign of the anisotropy changed, indicating that asymmetric xenon-xenon collisions along the channel length became more important at higher pressures. The authors explained their observations in terms of an zxiallysymmetric chemical shift tensor with components $\sigma_{x x}, \sigma_{y y}$, and $\sigma_{z z}$ in the principle axis system of the crystallite as shown in Figure 3.3. The xenon-xenon collisions, represented by $\sigma_{z z}$, become more important and eventually dominate the surface interactions (represented by $\sigma_{x x}$ and $\sigma_{y y}$ ) at higher xenon pressures. Ripmeester [101] has also discussed his observations of CSA patterns in xenon clathrates where xenon is trapped in the asymmetric environments of host molecules (see Clathrate section below).

Ripmeester presented a model [101] to explain the observation of the anomalous sign of the line shape anisotropy that is opposite to the cavity asymmetry. For instance, if the cavity shape is oblate with the $\sigma_{z z}$ symmetry axis being the shortest 


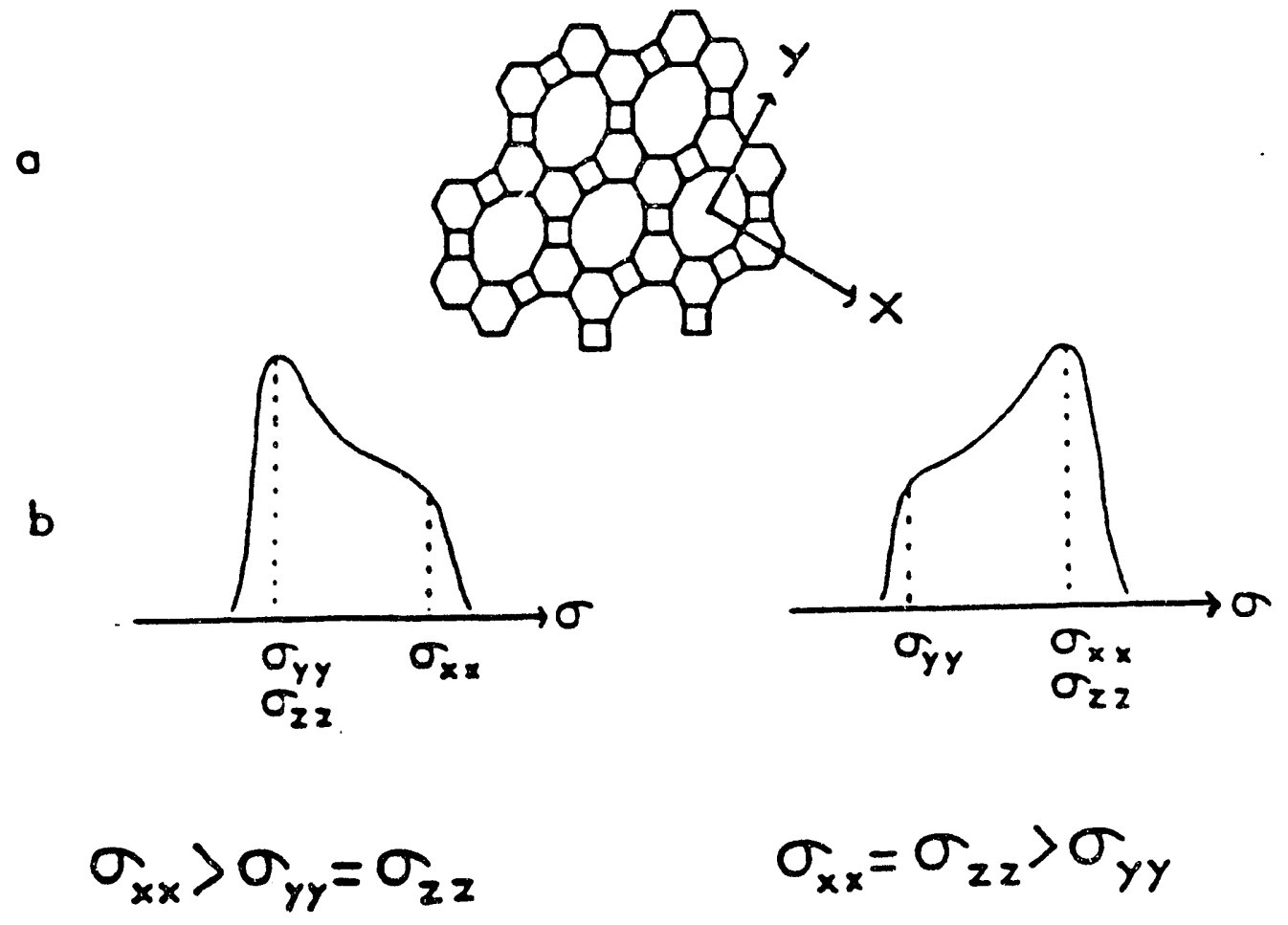

Figure 3.3: (a) Schematic drawing of the SAPO-11 molecular sieve. (b) Representative ${ }^{129} \mathrm{Xe}$ chemical shift anisotropy patterns at low and high xenon pressures. At low concentrations of xenon, $\sigma_{x x}>\sigma_{y y}, \sigma_{z z}$ and the line shape has a negative anisotropy, while at high xenon concentrations $\sigma_{z z}>\sigma_{x x}, \sigma_{y y}$ and the sign of the anisotropy changes. Adapted from Fraissard and coworkers [100]. 
for instance, this would normally imply a line shape with the $\sigma_{z z}$ component to lower field, a negative anisotropy. However, one finds the opposite to be true. Ripmeester argued that by assuming the xenon is moving, and that the CSA Tensor is axially symmetric at each point on the surface of the cage, by averaging over the cage surface one obtains the observed asymmetry.

\subsubsection{Metal Zeolites}

Metal zeolite studies are aimed at trying to improve the methods of preparation of these important catalysts, as well as to understand the mechanisms underlying their function. Fraissard and coworkers have studied the dispersion of platinum particles in Na-Y zeolite. They argued that xenon NMR could be used to give a qualitative idea of the average size of the metal particles when combined with hydrogen adsorption measurements [102], especially in cases where the particles are too small to be detected by Electron Microscopy (6-10 $\AA$ diameter). Chmelka et al. $[103,104]$ have studied the preparation of $\mathrm{Pt} / \mathrm{Na}-\mathrm{Y}$ catalysts and determined that the optimal calcination (treatment under flowing oxygen gas) temperature to create a highly-dispersed metal catalyst is approximately $300^{\circ} \mathrm{C}$. Above this temperature, the platinum particles migrate away from the supercages into the small, inaccessible sodalite cages. Xenon can detect the distribution of species such as $\mathrm{H}_{2}, \mathrm{O}_{2}$ or $\mathrm{CO}$ adsorbed onto the platinum particles in a zeolite crystallite [105]. At room temperature, the gas molecules adsorb onto the first platinum particle they encounter, as shown by 2 peaks in the ${ }^{129} \mathrm{Xe}$ NMR spectrum, while at $300{ }^{\circ} \mathrm{C}$, the gas molecules migrate, which gives rise to a more homogeneous distribution of adsorbed molecules over the zeolite crystallite. Fraissard finds that $\mathrm{CO}$ adsorbs with the stoichiometry of one molecule per particle, while hydrogen and oxygen adsorb as 2 atoms per platinum particle [105]. 


\subsubsection{Exchanged Cations}

The easily-exchanged cations in the zeolite framework have a dramatic effect on the various properties of zeolites such as sorption, gas separation and catalysis [90]. ${ }^{129} \mathrm{Xe}$ NMR has been used to characterize exchanged cations to describe their location and number in the zeolite that depend on exchange conditions and subsequent treatment procedures. For instance, in $\mathrm{Ca}-\mathrm{A}$ zeolite the first 4 or 5 exchangeable calcium cations are associated with the supercage, as can be seen by their interaction with xenon, while the remaining calcium ions (up to 2 additional cations) reside outside the supercage [106]. Early experiments by Fraissard seemed to indicate an electric field dependence on the chemical shift of ${ }^{129} \mathrm{Xe}[107]$ when the cations are located inside the supercages. Below $60 \%$ exchange, $\mathrm{Mg}$ or $\mathrm{Ca}$ does not show such an effect and the ${ }^{129} \mathrm{Xe}$ chemical shift is linear with increasing xenon concentration. This result plus X-ray data indicated that the cations are not located in the supercages except in highly exchanged samples. More recently, Cheung [95] has reinterpreted the effects of cations on the xenon chemical shift, attributing the nonlinear xenon chemical shift behavior to chemical exchange of xenon between adsorption sites and the gas phase. Nonetheless, Fraissard's qualitative interpretation of cation location in $\mathrm{Mg}$ and $\mathrm{Ca}$ exchanged zeolites is still correct.

Fraissard has also observed rubidium cations in zeolites exchanged for the first time without the presence of water. Normally, exchange preparations are performed under aqueous conditions or at least with some water adsorbed in the zeolite. But Fraissard showed [108] that it is possible, at high temperatures to exchange Rb in $\mathrm{Na}-\mathrm{Y}$ and $\mathrm{Na}-\mathrm{X}$ zeolites, although with a rather slow rate as shown by the multiple peaks in the ${ }^{129} \mathrm{Xe}$ spectra he observed. Xenon NMR has also been used to look at ruthenium [109] and $\mathrm{NiNa}-\mathrm{Y}$ exchanged zeolites [110, 111]. 


\subsubsection{Low Temperature Studies}

An interesting new development has been the extension of ${ }^{129} \mathrm{Xe}$ NMR studies of zeolites to low temperatures. Primarily through the work of Cheung [95], a better understanding of xenon's interaction with the cations inside zeolites has allowed a more comprehensive theory of the chemical shift in a variety of materials and conditions. He has proposed a model based on fast chemical exchange that explains the chemical shift trends observed earlier in a variety of different zeolite systems. Working at $144 \mathrm{~K}$ with a liquid nitrogen/ $n$-pentane temperature bath, he has observed gas-liquid phase transitions in $\mathrm{Na}-\mathrm{Y}$ zeolite at high xenon loadings [95]. He also studied pore size distributions in amorphous materials such as silica and alumina. [112]. He observed the same effects at low xenon loadings, namely an increase of the chemical shift as the pressure is lowered, as is observed in cation-exchanged zeolites (see Figure 3.2). This result has led to Cheung's description of the xenon chemical shift in terms of chemical exchange, without resorting to special effects such as $\delta_{E}$, xenon chemical shifts due to electric field effects, as proposed by Fraissard [113]. Cheung has also looked at Fluid Cracking Catalysts (zeolites grown from clay substrates for reasons of cost) and their degradation after use [114]. Possible future studies include looking at xenon phase changes mediated by surface interactions of different materials.

\subsubsection{Diffusion Studies}

${ }^{129} \mathrm{Xe}$ NMR has recently been used to study diffusion in zeolites. Pfeifer and coworkers [115] have used the traditional method of pulsed field gradients [116] to measure the diffusion constant of xenon inside $\mathrm{NaCa}-\mathrm{A}$ zeolite. They measured the diffusion constant to be $1.5 \times 10^{-5} \mathrm{~cm}^{2}-\mathrm{sec}^{-1}$ at a loading of 1.5 xenon atoms 
per supercage. The authors mentioned that one must be careful in analyzing such systems because xenon can diffusion through several zeolite crystallites during an NMR time period and the interparticle diffusion may make a sizeable contribution to the intraparticle diffusion constant one wants to measure [117]. A recent molecular dynamics study showed the diffusion in Na-Y zeolite to be substantial $\left(1.86 \times 10^{-5}\right.$ $\mathrm{cm}^{2}-\mathrm{sec}^{-1}$ ) even at loadings of 4 xenon atoms per cage [118]. Fraissard and Kärger proposed a model to describe xenon's mobility in Pt zeolites which can be used to study the location and distribution of $\mathrm{H}_{2}$ molecules adsorbed in the zeolite crystallite [119]. Dybowski and coworkers [120] showed an interesting application xenon NMR to measure the intercrystallite diffusion of water in $\mathrm{NiNa}-\mathrm{Y}$ zeolite by placing a layer of zeolite that had been evacuated at $100^{\circ} \mathrm{C}$ over a layer of zeolite evacuated at room temperature. They observed the coalescence of the NMR signal with a simple exponential time-dependence.

\subsubsection{Coking of Zeolites}

Xenon NMR has also been applied to the study of coking (carbonaceous deposits due to insufficient hydrocarbon oxidation) in zeolites. By considering $\delta_{S}$, the chemical shift at zero xenon concentration, an indication of the reduction of space inside the zeolite pore spaces was deduced [121] to be up to $20 \%$ reduction in volume for heavily coked propylene in $\mathrm{Na}-\mathrm{Y}$ zeolite. The formation of macropores with diameters larger than $25 \AA$ due to coking on the exterior surface of the zeolite crystallites was also observed. Miller it et al. [122] used a variety of techniques including xenon NMR to describe their coked H-Y zeolites prepared at different temperatures and with different crganic reagents. They found that two different sized pore spaces resulted in their highly coked samples as shown by Ar adsorption studies. Xenon $\overline{N M} \bar{R}$ was not sensitive to the different sized pore spaces, indicating a fast exchange 
of xenon between the pores that are in close proximity. Dybowski and coworkers investigated ZSM-5 and HZSM-5 zeolites and found that the coking character was radically changed by the addition of hydrogen which altered the acidity of the zeolite in HZSM-5. In the case of ZSM-5, coking blocked the zeolite channels severely, limiting xenon's access to much of the zeolite volume as seen by the much reduced adsorption of xenon. Dybowski noted that application of ${ }^{129} \mathrm{Xe}$ NMR methods could give qualitative information but it is risky to assume that empirical relations such as those derived by Demarquay [96] or Derouane [97] can give accurate values for structural quantities such as the pore volumes or diameters.

\subsubsection{Zeolite-Guest Systems}

Recent experiments on zeolite-guest systems have obtained interesting information on guest distributions and their location inside the zeolite framework. For instance, xenon NMR has been used to look at organic molecules such as benzene, trimethylbenzene and $n$-hexane adsorbed inside $\mathrm{Na}-\mathrm{Y}$ zeolite [123]. Xenon is sensitive to the type and concentration of the adsorbed guest. Despite expectations that benzene would have a large interaction with xenon due to polarizability considerations, $n$-hexane has the largest interaction among the three guests as shown by the xenon chemical shift. The chemical shift also increases with guest concentration for all guest probably due to the reduction in the free volume inside the supercage.

Using ${ }^{129} \mathrm{Xe}$ combined with multiple quantum NMR, one can look at the distributions of benzene and hexamethylbenzene in zeolites [124]. Because xenon is very mobile, it is sensitive to distributions on a macroscopic scale on the order of $10 \mu \mathrm{m}$. Xenon NMR has shown that thorough heat treatments are necessary in order to create very homogeneous distributions of organic guests on such a length scale [125]. Meanwhile, MQ NMR [126] allows one to essentially "count" spin clusters which are 
coupled via the dipole-dipole interaction. Multiple quantum NMR has been used to show that the distribution of benzene molecules inside $\mathrm{Na}-\mathrm{Y}$ zeolite is homogeneous on a microscopic scale [124].

\subsection{Coals}

Xenon NMR has recently been used to characterize small pore spaces inside coals and amorphous carbon. Following along similar methods applied to zeolites, Ryoo and coworkers [127] studied several types of amorphous carbon and concluded that xenon was useful in studying such materials and might provide a simple means to characterize them. As in the zeolite work of Fraissard and others, Ryoo found that the xenon chemical shift was a linear function of the loading pressure and that the value extrapolated to zero pressure was indicative of the nature of the surface since it depends on the xenon-surface interaction. However, Ryoo did not find a relationship of the chemical shift to the pores sizes probably because the pore spaces were too large (about $8 \AA$ from $t$-plots) to show a sizeable effect. Differences in the pore spaces were observed in Illinois \# 6 coals $[128,129]$ where two peaks in the ${ }^{129} \mathrm{Xe}$ NMR spectrum had different pressure dependences. Tsaio el al. [128] attributed the two peaks to the pores with primarily aliphatic and aromatic nature. The chemical shifts, extrapolated to zero xenon pressure, give values around $120 \mathrm{ppm}$ for both indicating similar xenon-surface interactions. Tsiao it et al. also feel this indicates similar sizes for the aliphatic and aromatic pores. Coal samples typically have broad lines due to the inhomogeneous nature of the material such as a large distribution of pore sizes and their chemical makeup. 


\subsection{Xenon in Solution}

Studies by Diehl and coworkers have shown that xenon is very sensitive to . changes in local order when dissolved in anisotropic solutions. When dissolved in liquid crystal solvents, ${ }^{129} \mathrm{Xe}$ and ${ }^{131} \mathrm{Xe}$ NMR show dramatic changes in resonant shift [130] and quadrupolar splittings [131] (respectively) as the liquid crystal-xenon solution goes from an isotropic to an anisotropic, (ordered) nematic phase. As shown in Figure 3.4, the ${ }^{129} \mathrm{Xe}$ shielding shows a sharp discontinuity across the isotropicnematic phase boundary that is due to the ordering of the liquid crystal director preferentially along (positive bulk anisotropic susceptibility) or perpendicular (negative bulk anisotropic susceptibility) to the applied magnetic field. The change in the chemical shielding is caused by the rotation of the liquid crystal director from a parallel orientation to perpendicular at $310 \mathrm{~K}$ as shown in the Figure. These effects can be attributed to interactions of the xenon with a non-spherically symmetric environment that should become larger with the liquid crystal ordering (lower temperatures). Diehl and coworkers have also examined the electric field gradient (efg) experienced by xenon in the liquid crystal environment in ${ }^{131} \mathrm{Xe}$ experiments. The quadrupolar splittings observed are proportional to the average efg experienced by the ${ }^{131} \mathrm{Xe}$ nucleus [131]. An anomalous result was obtained [132] when xenon was dissolved into a "critical" mixture of two liquid crystals (ZLI1132 and EBBA) with opposite electric field gradients that were expected to average to zero. However, 400 to $500 \mathrm{KHz}$ (depending on the temperature) splittings was observed, which were in fact larger than for either of the two pure components! The source of such an anomalous result is not understood. The authors agree that the effect is not due solely to the liquid crystal efg since the splitting does not increase monotonically as the temperature is decreased (the efg is proportional to the liquid crystal ordering). Other 


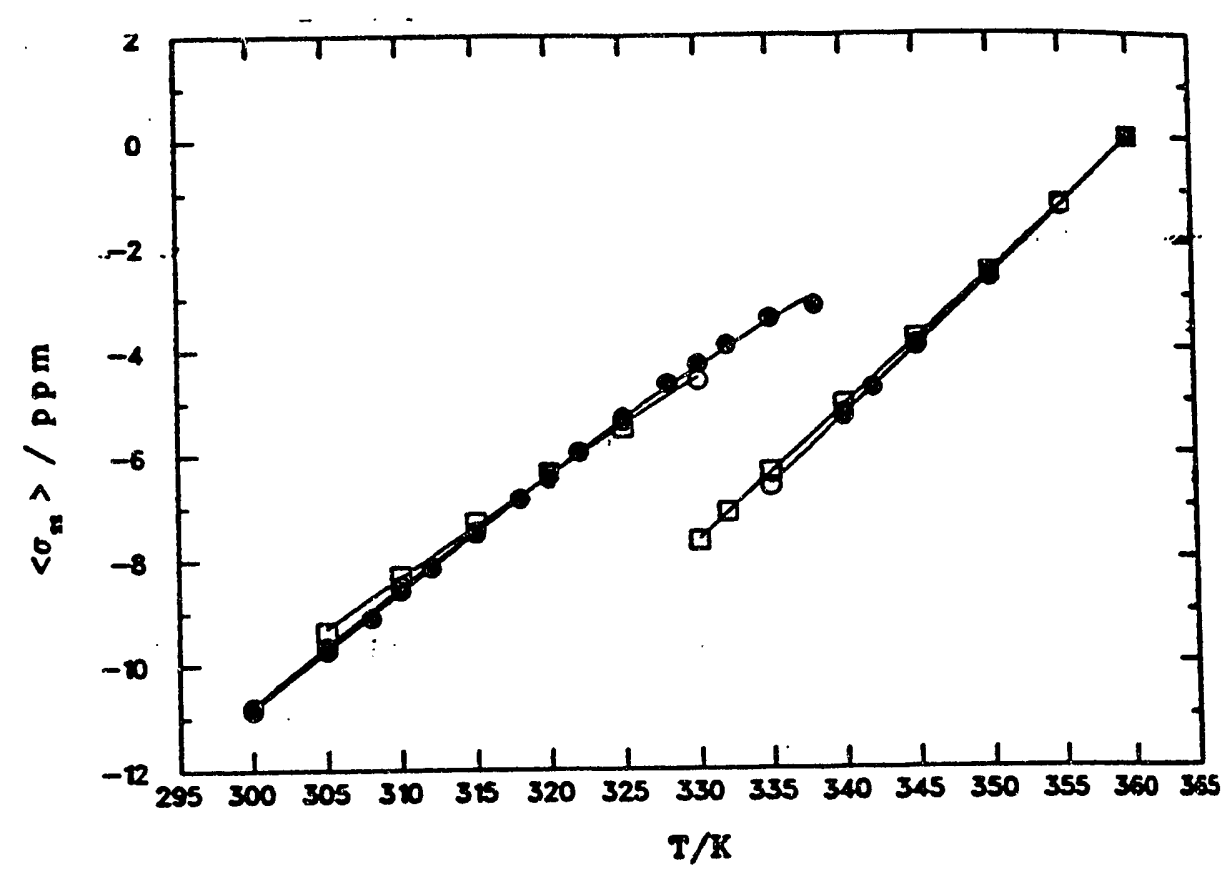

Figure 3.4: Xenon-129 in liquid crystal. The phase change is clearly seen as a break in the chemical shift values as the temperature is changed. Adapted from reference [130]. 
effects such as electron cloud polarization or van der Waals forces were suggested as possible explanations.

Several studies of xenon in solution have been made concerning the understanding of the xenon-solvent interaction and its influence on the chemical shift and relaxation rates of xenon. Stengle, Williamson and coworkers have investigated the relaxation of ${ }^{131} \mathrm{Xe}$ in a large variety of polar solvents [133] and have compared three theories and some Monte-Carlo calculations to explain their relaxation data. The theory which best fits their data is one due to Hertz [134] who considered the efg-induced relaxation due the solvent modeled as a collection of fluctuating point dipoles. A fundamental problem with all three theories is the determination of the correlation time since the relaxation rate depends on both the efg and $\tau_{c}$. The results of their study also showed that a unique correlation time based on solvent molecule tumbling was probably not possible. The xenon chemical shift was not affected by the electric dipole moment of the solvent molecules.

A new theoretical model has been developed by Reisse and coworkers which they have applied to a variety of xenon-solvent systems to correlate the ${ }^{129} \mathrm{Xe}$ chemical shift. They have shown that their theory describes the chemical shift better than so-called the "reaction field" model which has been used to correlate the chemical shift with a solvent function based on the index of refraction [135]:

$$
f(n)=\left(\frac{n^{2}-1}{2 n^{2}+1}\right)^{2} .
$$

For related molecules the reaction field model seems to predict the chemical shift behavior quite well, as in the series of straight chain alcohols studied by Stengle and coworkers [136]. Although water does not fit the model at all, Reisse and coworkers did not consider it in their paper. 


\subsection{Clathrates}

The low symmetry of xenon clathrate (inclusion) structures gives rise to chemical shift anisotropy patterns in the xenon NMR spectra [101]. Ripmeester has reported a new clathrate structure [137] and used xenon NMR to characterize it [138]. The new clathrate has a unit cell composition $M_{1} \cdot 2 M_{2} \cdot 3 M_{3} \cdot 34 H_{2} O$, where $M_{2}$ and $M_{3}$ are small cages of about $6 \AA$ in diameter, and $M_{3}$ is a large, oblong cavity. A "help-gas," methyl cyclohexane, was added to the xenon- $\mathrm{H}_{2} \mathrm{O}$ mixture to form the new clathrates. Other help gases are expected to work as well.

In xenon-hydrate clathrate, Ripmeester and coworkers [139] have studied the thermodynamics of xenon in the small and large cages. The small cage has tetrahedral symmetry since the xenon NMR spectrum shows a symmetric line shape. The large cage, however, has an asymmetry of $\Delta \sigma=\sigma_{\|}-\sigma_{\perp}$ of about 34 ppm [143]. Ripmeester comments that the source of the observed broadening changes from a homogeneous nature (where each xenon experiences the same asymmetric environment) to a heterogeneous broadening (where each xenon is frozen into its own asymmetric environment with a different asymmetric lineshape) as the temperature is lowered. In the latter case the observed lineshape is an isotropic average of orientations for the whole sample. The authors were able to obtain $\Delta \mu_{w}^{\circ}$, the chemical potential of the empty hydrate relative to solid ice. Their results is $1297 \mathrm{~J} /$ mole in accordance with several other studies using different methods. Xenon hydrate is stable at room temperature under a pressure of about $20 \mathrm{~atm}$. The ${ }^{129} \mathrm{Xe}$ NMR of a xenon clathrasil (a $\mathrm{SiO}_{2}$ "clathrate") was also reported by Ripmeester and coworkers [144]. 


\subsubsection{Cross-Polarization Experiments}

In Ripmeester's studies on xenon clathrates, proton-xenon cross-polarization (CP) was applied in order to decrease the signal averaging time, but they found that the short relaxation time in the rotating frame, $T_{1 \rho}$, caused the ratio of the cage occupancies to vary. At $77 \mathrm{~K}, T_{1 \rho}$ for protons is much longer. The $\mathrm{CP}$ experiment allows polarization transfer between spin species by matching their energies in the rotating frame $[16,140]$. The proton-xenon $\mathrm{CP}$ experiment is unusual because it involves coupling of two species which are physisorbed, and not chemically-bonded. The xenon's motion does not completely average out the dipole-dipole necessary to perform the $\mathrm{CP}$ experiment because xenon is trapped in the cages and does not undergo isotropic motion. However, the CP contact time is quite long, about 30 milliseconds and can be troublesome for some NMR probes. In Figure 3.5 a spectrum for the xenon-quinol clathrate is shown. Instead of the more common CP experiment using rotating frame polarization transfer during two simultaneous strong rf pulses, order can also be transferred using an adiabatic process (Adiabatic Demagnetization in the Rotating Frame [141]) to allow more efficient cross-polarization [142].

\subsection{Xenon in Polymers}

Recently, ${ }^{129} \mathrm{Xe}$ NMR was been applied to the study of polymers. Stengle and Williarnson [145] have adsorbed xenon into low density polyethylene (LDPE) at pressures of $8 \mathrm{~atm}$ and have observed a ${ }^{129} \mathrm{Xe}$ resonance at approximately $200 \mathrm{ppm}$ which they attribute to amorphous regions inside the solid polymer. Their results fit nicely with their previous work on xenon shifts in $n$-alkane solutions. Temperature dependent measurements by the authors demonstrate xenon's sensitivity to detect phase transitions in poly(ethyl)methacrylate. 


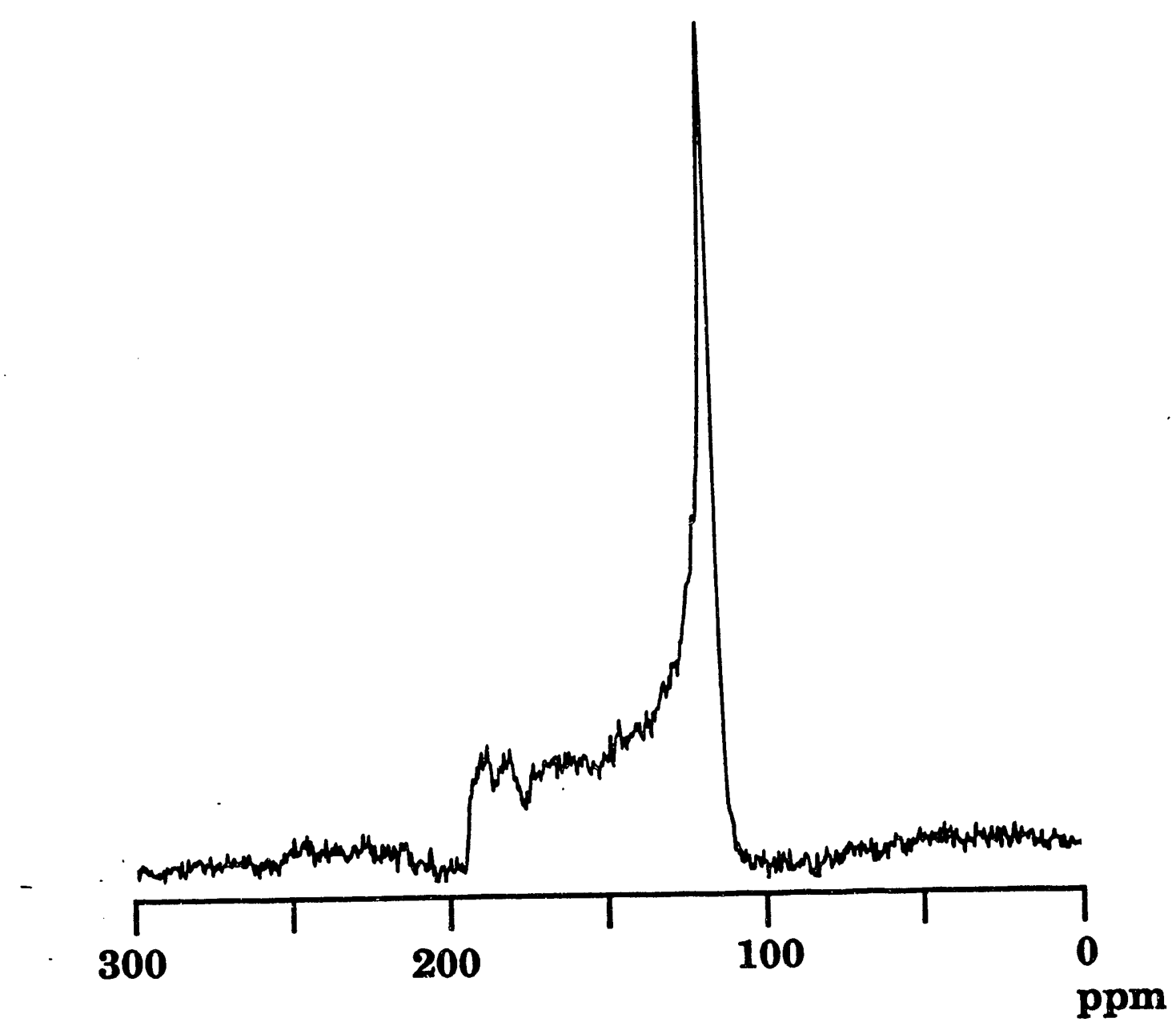

Figure 3.5: ${ }^{129} \mathrm{Xe}$ NMR spectrum of a xenon-quinol clathrate obtained using xenon-proton cross-polarization experiment using ADRF (see text). The signal-to-noise is increased because of the higher polarization of the protons and their shorter relaxation time. Spectrum results 80 accumulations with a recycle time of 60 seconds. 
Bronwstein it et al. [146] found that xenon line widths were sensitive to the particular environment of the amorphous polymer. They detected two lines in the ${ }^{129} \mathrm{Xe}$ spectrum indicating two distinct regions in their polystyrene-polyisoprene block copolymers. They presented evidence that the observed line width of up to $6 \mathrm{kHz}$ was due primarily to diffusion between the two polymer domains. The line widths increased as the domain size of the polymers were decreased. Calculations of the diffusion coefficient gave $D=3 \times 10^{-7} \mathrm{~cm}^{2} / \mathrm{sec}$ which is slightly less than measured values for nitrogen and argon. Kennedy [147] has examined rubber curing using xenon NMR and found that the rubber becomes more homogeneous due to the sulphur cross-linking as evidenced by a reduction in the numbers of features in the NMR spectrum.

\subsection{Xenon Compounds}

A large number of xenon-containing compounds have been synthesized, and characterized by several methods including ${ }^{129}$ Xe NMR [1], ever since the first observation that xenon could be reacted with fluorine to form $\mathrm{XeF}_{2}$ [148]. Early on, $\mathrm{CW}$ doubleresonance "spin-tickling" experiments on xenon compounds such as $\mathrm{XeOF}_{4}, \mathrm{XeF}_{2}$, and $\mathrm{XeF}_{2}$ were performed by irradiating the xenon resonance while observing the ${ }^{19} \mathrm{~F}$ NMR [149]. However, the first direct observations of ${ }^{129} \mathrm{Xe}$ were made by Seppelt and Rupp, in compounds including $\mathrm{XeF}_{4}, \mathrm{XeF}_{6}, \mathrm{XeO}_{3}, \mathrm{Xe}\left(\mathrm{OSeF}_{5}\right)_{2}, \mathrm{Xe}\left(\mathrm{OTeF}_{5}\right)_{2}$, and others [150]. More recent work by Schrobilgen and coworkers has gone far to understand the structure and bonding of xenon in a large variety of interesting xenon compounds. Xenon chemical shifts can be understood in terms of the formal oxidation state of xenon, ionic bond character and solvent effects [151]. The synthesis of xenon compounds with metal ligands, $\mathrm{XeF}_{2} \cdot n \mathrm{WOF}_{4}$ and $\mathrm{XeF}_{2} \cdot n \mathrm{MoOF}_{4}$ [152], and 
xenon-nit:ogen bon ${ }^{\prime} ;$ in $\mathrm{FXeN}\left(\mathrm{SO}_{2} \mathrm{~F}\right)_{2}$ [153] are examples of some of the interesting chemistry apparent in their work.

Two major trends describe the chemical shifts. Except for $\mathrm{Xe}^{l V}$ (xenon with formal charge of +4) compounds such as $\mathrm{XeF}_{4}$ and $\mathrm{XeF}_{3}^{+}$, the chemical shift increases with the oxidation state (decreasing formal charge): $\mathrm{Xe}^{V I I}<\mathrm{Xe}^{I V}<\mathrm{Xe}^{V I}<\mathrm{Xe}^{I I}$ as was suggested by Jameson and Gutowski in their calculations [9]. Oxygen ligands tend to increase the chemical shift, probably due to the ionic bond character of $\mathrm{Xe}^{+}-\mathrm{O}^{-}$formation. Also evident in the spectra are the highly resolved spin-spin couplings to ${ }^{19} \mathrm{~F}$, which can be as large as $8 \mathrm{kHz}$, and to other ligands, although fluorine is the most prevalent. Axial ligands have much larger spin-spin couplings than equatorial ligands which can have zero or even negative $J$ couplings [1]. Xenon chemical shifts show a sensitivity to the polar character of its solvents. Schrobilgen suggests that $\mathrm{XeF}_{2}$ may be solvated by weak bonds through the fluorine ligand such as:

$$
F-X e-F--H-F
$$

Naumann and Tyrra [154] report the first stable xenon-carbon bond in $\mathrm{Xe}\left(\mathrm{CF}_{3}\right)_{2}$ which they charactericed with xenon NMR. And recently, Turowski and Seppelt [155] have synthesized the first xenon polymer which is insoluble in all common solvents and stable to $80^{\circ} \mathrm{C}$. The monomer has the formula $\left(-\mathrm{Xe}-\mathrm{O}-\mathrm{TeF}_{4}-\mathrm{O}-\right)_{n}$.

\subsection{Xenon with Biological Molecules}

Two paper; have appeared concerning xenon in biological molecules. Stengle, Williamson and coworkers [156] have extended their studies of xenon in various solvents to include myoglobin, $(\mathrm{Myr})_{2}$ lec lipid bilayers and Torpedo californica electric fish membranes in aqueous solutions. The xenon in these systems undergoes rapid 
exchange on the relevant NMR time scale between the solution and association with the protein. In the case of myoglobin, the authors estimate that approximately 10 xenon atoms are associated with each protein molecule in addition to the xenon binding site known from X-ray data. In (Myr) $)_{2}$ lec vesicles, xenon at $35^{\circ} \mathrm{C}$ shows a distinct peak from the aqueous solution in addition to the peak associated with the protein. These peaks coalesce at $50^{\circ} \mathrm{C}$, as the xenon exchanges rapidly.

Tilton and Kuntz have studied several xenon-protein systems and determined the rates of association by measuring the line width as a function of temperature and protein concentration [157]. They determined that the association rate is approximately $6 \times 10^{7} M^{-1} s^{-1}$ for xenon with methemoglobin and $1 \times 10^{7} M^{-1} s^{-1}$ for xenon with metmyoglobin. The xenon chemical shift was found to be sensitive to the iron oxidation and spin states, with the so-called "met" state $\left(\mathrm{Fe}^{3+}\right)$ more deshielded by approximately 5-10 ppm from the other iron states of myoglobin. 


\section{Chapter 4}

\section{Xenon as a Probe of Organic Molecules Adsorbed in Na-Y}

\section{Zeolite}

As was discussed in Chapter $1,{ }^{129} \mathrm{Xe}$ is a useful probe in NMR experiments because of its chemical inertness, its large polarizability, and its relative ease of detertion. Due to its highly favorable NMR characteristics of high natural abundance and large chemical shift range, ${ }^{129} \mathrm{Xe}$ has been used as a sensitive probe of its chemical environment in zeolites and other materials as discussed in Chapter 3. For instance, from chemical shift data, ${ }^{129} \mathrm{Xe}$ NMR has been used to calculate void space volumes of various zeolites [96], and to investigate the dispersion of metal clusters among the zeolite supercages $[105,158]$.

It is of interest to investigate whether xenon can be used to study different species adsorbed in the zeolite structure. Using ${ }^{129} \mathrm{Xe}$ NMR, Fraissard has studied the effect of co-adsorbed water molecules on the void volume of Na-Y supercages [159]. Previous experiments by Lechert and coworkers were concerned with the second moment of 
proton NMR spectra for adsorbed benzene molecules in faujasite-type zeolites [160]. Workers in the Pines group have studied adsorption of hexamethylbenzene in $\mathrm{Na}-\mathrm{Y}$ zeolite by ${ }^{129} \mathrm{Xe}$ NMR and multiple-quantum spectroscopy [124, 125]. The purpose of this chapter is to describe experiments which characterize xenon's interactions with the zeolite support and to study the adsorption of different guest molecules inside the zeolite structure. It is found that ${ }^{129} \mathrm{Xe}$ NMR is sensitive to the identity of the adsorbed species, to its concentration, and possibly to its molecular arrangement inside the zeolite supercage.

\subsection{Sample Preparation and Data Collection}

Samples were prepared by first heating $\mathrm{Na}-\mathrm{Y}$ zeolite at $400{ }^{\circ} \mathrm{C}$ under vacuum $\left(10^{-6}\right.$ torr) overnight to desorb water or other contaminant molecules that might be trapped inside. Guest molecules of various sizes and shapes ( $n$-hexane, benzene, and trimethylbenzene) were adsorbed into separate samples of Na-Y zeolite either by addition of some of the liquid organic guest species, in the case of trimethylbenzene, or by distillation in the cases of $n$-hexane and benzene. Samples were then heated typically for 1-3 hours above the normal boiling point temperature of the bulk guest species to ensure its even distribution throughout the zeolite sample volume. Heat treatment has been shown to play a role in the dispersion of the adsorbed guest in the zeolite [125]. Room temperature xenon adsorption isotherms were measured both before and after the guest molecules were introduced into the zeolite (see Appendix A for a discussion of the isotherms). Helium gas was used to determine the dead volumes of the samples.

${ }^{129} \mathrm{Xe}$ spectra were obtained primarily on a Nicolet spectrometer operating at $49.8 \mathrm{MHz}$. FIDs were acquired following a single $90^{\circ}$ pulse at approximately $0.5 \mathrm{sec}-$ 
ond intervals. To ensure adequate signal-to-noise ratios, approximately 2000 to 4000 scans were accumulated for each spectrum, depending on the xenon pressure. Chemical shift data are relative to a 5.0 atm xenon gas sample [2] (referenced to zero xenon pressure), which is at a lower resonance frequency than that of the samples.

\subsection{Results}

Figure 4.1 illustrates the basic theme of this work. The ${ }^{129} \mathrm{Xe}$ NMR adsorption signal consists of a single, symmetrical line with a width of approximately $5 \mathrm{ppm}$. Although the line is partially motionally-averaged [2], the observed line width is probably due to chemical shift anisotropy. The position of the line is a function of both the guest species and its concentration, as well as the density of the xenon inside the supercages of the zeolite. The schematic drawing in Figure 4.1A, represents a xenon pressure of 100 torr, which corresponds (on the average) to one xenon atom per two Na-Y supercages, and the resulting NMR spectrum. The chemical shift is $65.7 \mathrm{ppm}$ at 100 torr xenon pressure. Adsorption of approximately one benzene molecule per supercage shifts the ${ }^{129} \mathrm{Xe}$ resonance downfield to $81.7 \mathrm{ppm}$ as shown in Figure 4.1B. Increasing the xenon pressure to 600 torr further increases the chemical shift to $111.5 \mathrm{ppm}$ (Figure 4.1C).

The full set of chemical shift data for adsorbed benzene in various quantities $(0.5$, 1 , and 2 molecules per supercage) as a function of the equilibrium xenon pressure is shown in Figure 4.2. All chemical shift plots are linear functions of the xenon pressure with slopes of approximately $0.060 \mathrm{ppm}$ per torr of xenon pressure in agreement with previous data on xenon in $\mathrm{Na}-\mathrm{Y}$ zeolite [2]. The slopes are similar for different amounts of adsorbed benzene, while the chemical shift increases with the concentration of benzene molecules at constant xenon pressure. 

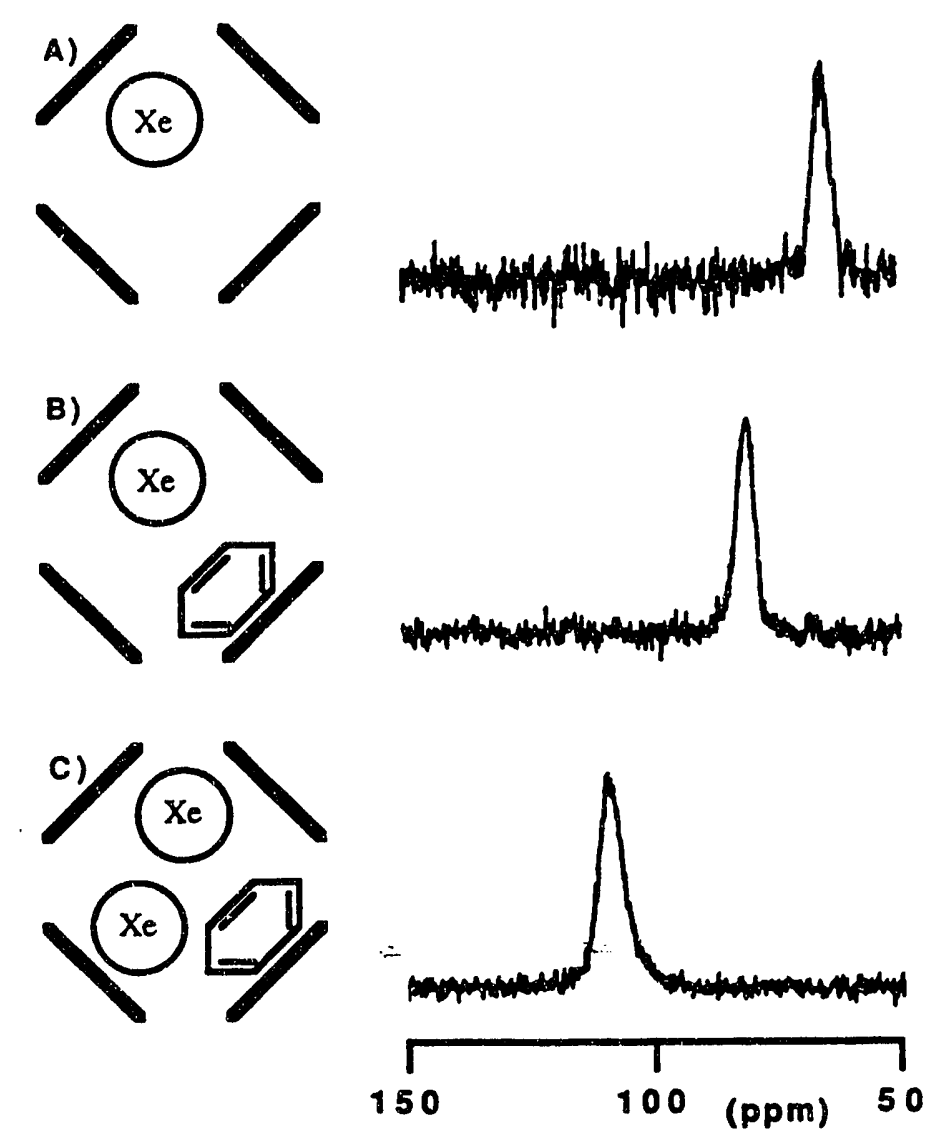

Figure 4.1: Schematic illustration of a $\mathrm{Na}-\mathrm{Y}$ supercage with adsorbed (A) xenon (100 torr), (B) xenon (100 torr) plus one benzene molecule per cage, and (C) xenon (600 torr) plus one benzene molecule per cage on the left, and the resulting xenon-129 NMR spectrum for each on the right. 


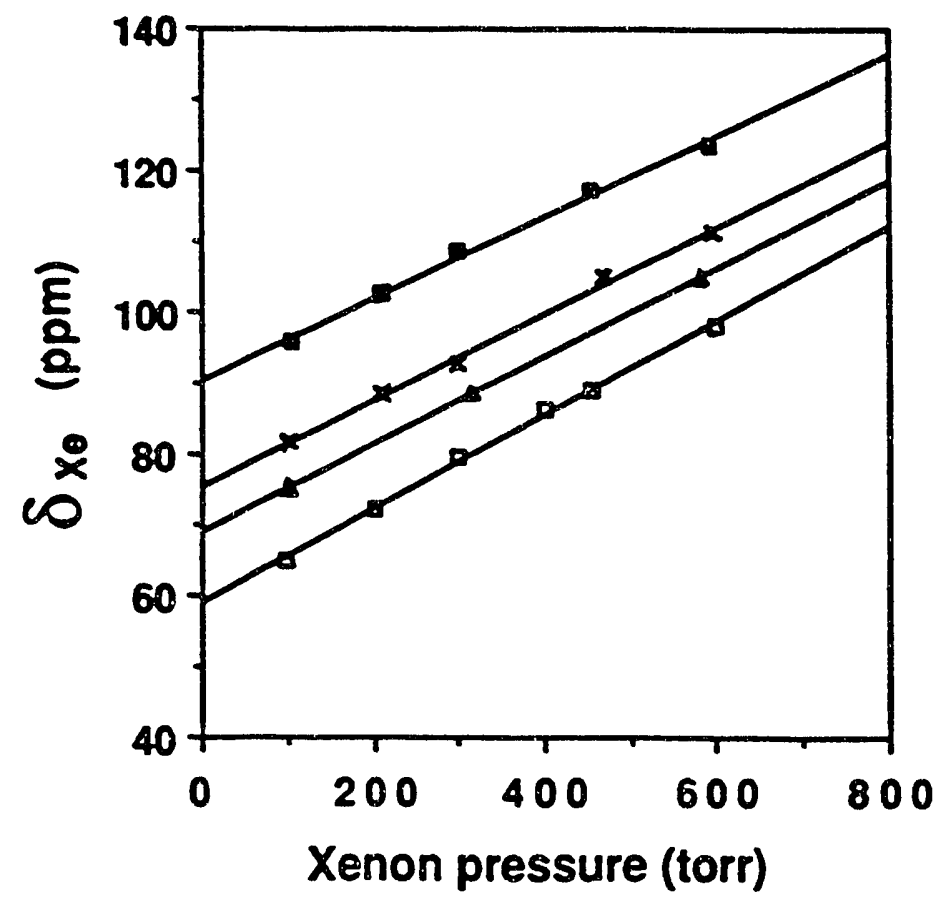

Figure 4.2: The full chemical shift data (in units of ppm versus xenon pressure) for adsorbed benzene in concentrations of $0.0(\square), 0.5(\Delta), 1(x)$ and $2(\square)$ molecules per supercage. 
Xenon adsorption isotherms in $\mathrm{Na}-\mathrm{Y}$ zeolite are shown in Figure 4.3 for various quantities of guest species in the zeolite supercages.

The Figure displays the effect of guest species loadings corresponding to an average of $0.5,1$, and 2 molecules per zeolite supercage compared with the bare Na-Y zeolite sample. The quantity of xenon adsorbed is plotted against the equilibrium xenon pressure measured at room temperature. The isotherms indicate that adsorption of xenon in the presence of adsorbed guest species is similar in all cases to that of the bare $\mathrm{Na}-\mathrm{Y}$ support except for xenon pressures above 300 torr where the effect of the adsorbed molecule becomes significant and the xenon adsorption starts to show some saturation. This feature is most pronounced in Figure 4.3 for the sample containing (on the average) two trimethylbenzene molecules per zeolite supercage. The xenon adsorption isotherm is significantly different from that of xenon adsorbed in the bare $\mathrm{Na}-\mathrm{Y}$ sample. Smaller concentrations of trimethylbenzene show less deviation than the case of 2 molecules per supercage. This deviation is also apparent for the $n$-hexane and benzene samples at high guest molecule concentration, but to a slightly lesser extent than for the trimethylbenzene samples.

To more directly compare the variation of xenon-129 chemical shift versus xenon concentration for the various loadings of guest molecules, data such as those for benzene in Figure 4.2 can be replotted using the xenon isotherms to convert from xenon equilibrium pressure to xenon concentration (number of xenon atoms adsorbed per gram Na-Y zeolite). Figure 4.4 shows the chemical shift data for the different guest species at concentrations of $0.5,1$ and 2 molecules per supercage, versus the xenon concentration (as number of xenon atoms per gram of zeolite).

The xenon pressure range used in this study (100 to 600 torr) corresponds to xenon concentrations of approximately 0.5 to 3 xenon atoms per supercage. In Figure 4.5 the data have been replotted by grouping the samples by guest concentration 


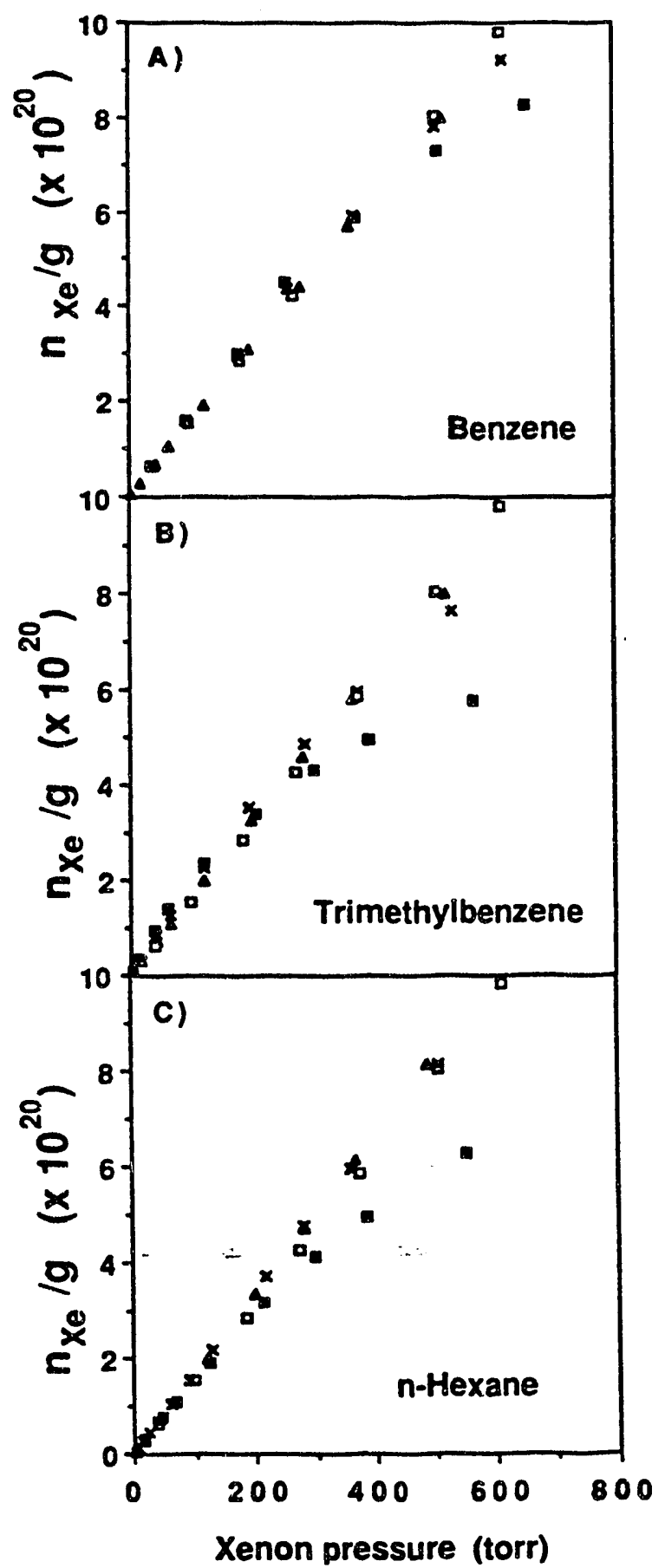

Figure 4.3: Xenon isotherms at $T=21^{\circ} \mathrm{C}$ in empty Na-Y zeolite ( $\square$ ) and with adsorbed guest species in concentrations of $0.5(\Delta), 1(x)$, and $2(\boldsymbol{*})$ guest molecules per supercage. Guest species: (A) benzene, (B) trimethylbenzene, and (C) n-hexane. 


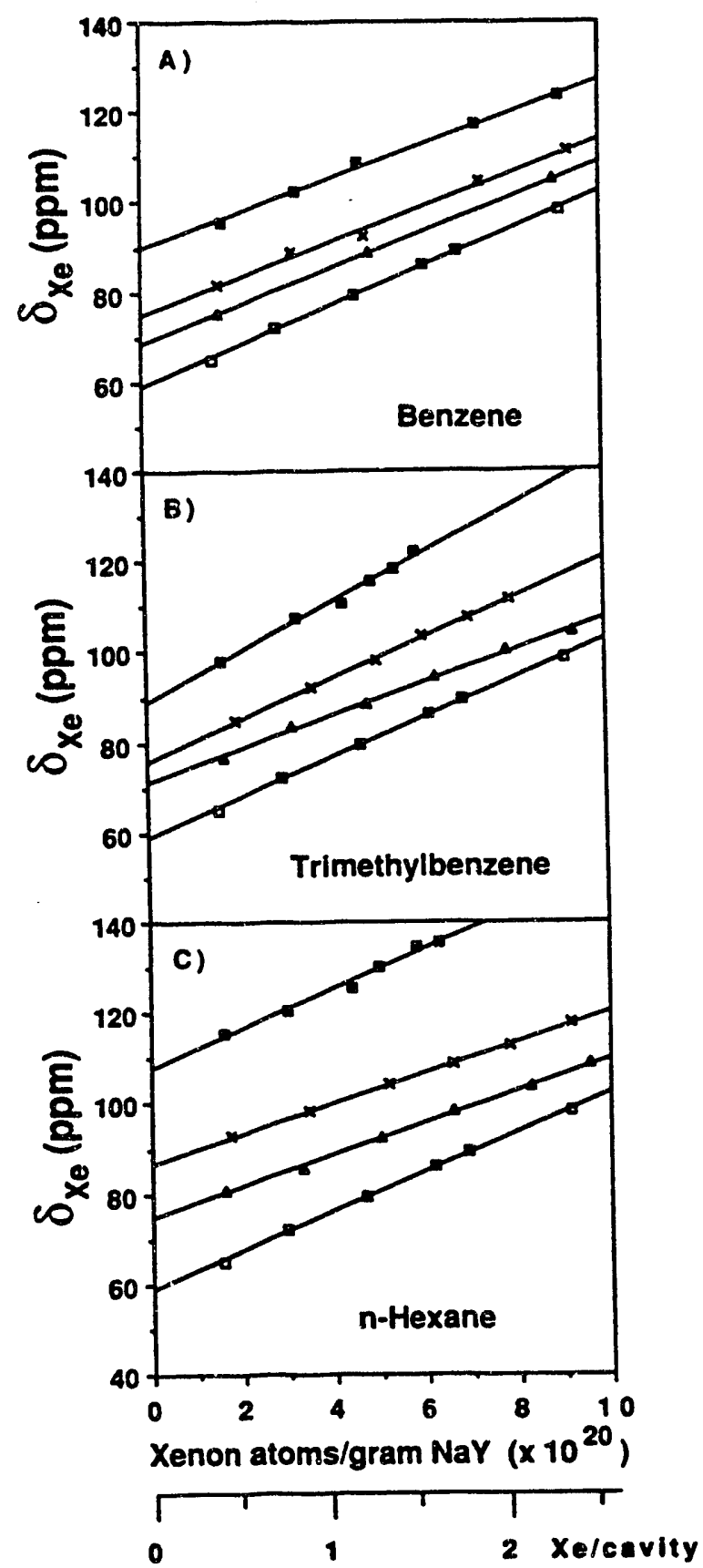

Figure 4.4: ${ }^{129} \mathrm{Xe}$ NMR chemical shift versus xenon concentration (xenon atoms per gram of zeolite and atoms per supercage) at $T=21^{\circ} \mathrm{C}$ for adsorbed guest species in $\mathrm{Na}-\mathrm{Y}$ zeolite for concentrations corresponding to $0.0(\square), 0.5(\triangle), 1(x)$, and $2(\square)$ guest molecules per cavity. Guest species: (A) benzene, (B) trimethylbenzene, and (C) n-hexane. 
instead of guest species. The $n$-hexane samples show the largest increase in the chemical shift of xenon with respect to the bare $\mathrm{Na}-\mathrm{Y}$ zeolite sample for all guest molecule concentrations, while the trimethylbenzene and benzene samples have similar but smaller increases over the bare $\mathrm{Na}-\mathrm{Y}$ case. It is evident that the slope of the lines connecting the chemical shift values depends on the concentration of the guest molecules in the zeolite, and that the slopes are different for different guest molecules.

\subsection{Discussion}

Due to the large size of the channels connecting zeolite supercages, xenon is essentially free to move among many supercages during the time period relevant to the NMR experiment $[2,125]$. This is evidenced by the appearance of a single line in the NMR spectrum of xenon-129, even in the case where there is less than one guest molecule, on the average, per supercage. Such is not the case for heterogeneous distributions of guest molecules because xenon may experience different environments in the sample: the two environments (empty cages versus cages with guest molecules) may be too far apart to be rapidly averaged on the NMR time scale [125]. But for all cases of dispersed distributions, we find the lines of the xenon resonance to be narrow, with no significant broadening at room temperature even due to the cations and paramagnetic impurities which are present in the zeolite lattice.

Jameson and Jameson have shown that the xenon-129 chemical shift can be written as a sum of contributions corresponding to isolated xenon atoms plus collisions between the xenon atoms, the most important of which are two body collisions [11]. Fraissard and coworkers have extended this idea by adding terms which take into account the interaction of xenon with the zeolite surface, exchangeable cations, metal 


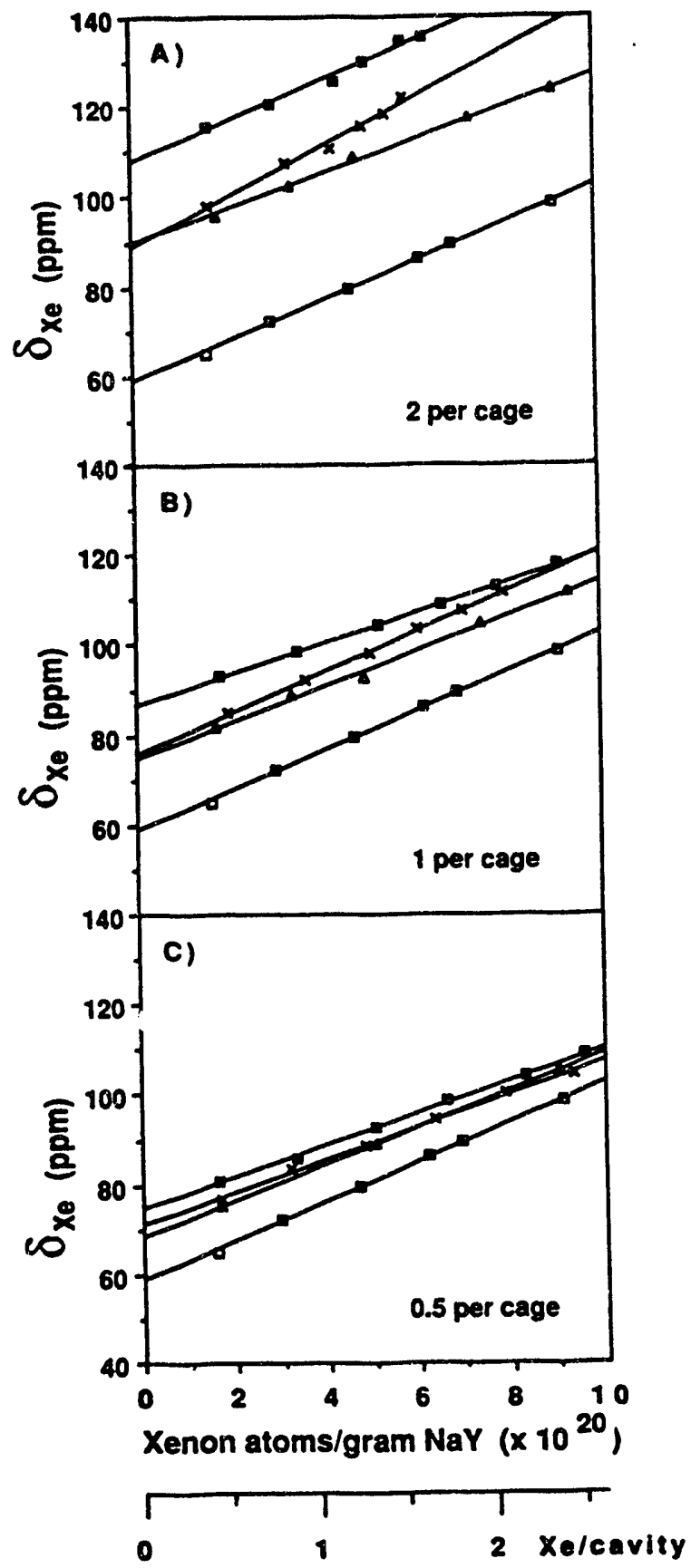

Figure 4.5: Xenon-129 NMR chemical shift versus xenon concentration (xenon atoms per gram of zeolite and atoms per supercage) at $T=21^{\circ} \mathrm{C}$ for adsorbed guest species in Na-Y zeolite: none $(\square)$, benzene $(\Delta)$, trimethylbenzene $(x)$, and $n$-hexane $(\boldsymbol{a})$, for concentrations corresponding to: concentrations corresponding to (A) $2,(B) 1$, and (C) 0.5 guest molecules per cavity. 
particles included in the supercages, or adsorbed water $[159,161]$. We can similarly add another term to describe the interaction of xenon with organic guest molecules inside the zeolite supercages.

By extrapolating the chemical shift values to zero xenon pressure, the effect of xenon-xenon collisions becomes negligible, leaving the xenon-zeolite surface and the xenon-guest molecule terms. In Table 4.1, values of the xenon-129 chemical shift $\delta_{X e}$ are shown for all samples, extrapolated to zero xenon pressure. For empty Na-Y zeolite, the xenon- 129 chemical shift is $58.9 \pm 0.5 \mathrm{ppm}$ corresponding to the xenon-zeolite surface contribution to thie chemical shift, which is in agreement with previous work by Ito and Fraissard [2]. The benzene and trimethylbenzene samples show similar values of $\delta_{X e}$ at all guest species concentrations. At a concentration of 2 molecules per supercage, $\delta_{X e}$ is $89.7 \pm 0.1 \mathrm{ppm}$ for benzene and $89.0 \pm 1.3 \mathrm{ppm}$ for trimethylbenzene which are both much larger than the empty Na-Y case. $n$-Hexane shows the largest value of the chemical shift of xenon in our samples, $107.7 \pm 1.2 \mathrm{ppm}$ for approximately 2 guest molecules per supercage at zero xenon pressure. Small errors in the slope and intercept given in Table 4.1 are an indication that the data are linear. The error of any particular chemical shift value is typically $0.5 \mathrm{ppm}$.

We can draw some conclusions regarding the location of the guest molecules inside the supercages. As has been suggested by Lechert and coworkers in NMR studies [160], and by Mallmann and Barthorneuf [162] from their infrared work, benzene is probably attached to the walls of the supercages near cation sites, although it can rotate freely about its six-fold axis. Trimethylbenzene, having a geometry similar to benzene, and practically the same chemical shift value at zero xenon pressure in these experiments, is most likely attached in a similar manner to the zeolite supercage walls. $n$-Hexane, on the other hand, has a much larger interaction with xenon. One possible scenario to explain the large xenon chemical shift is that the $n$-hexane 


\begin{tabular}{lccc}
\hline & $\begin{array}{c}\text { concn, } \\
\text { molecules/ } \\
\text { gugest molecule }\end{array}$ & $\begin{array}{c}\delta_{X_{e}} \text { at } \\
P_{X_{e}}=0, \mathrm{ppm}\end{array}$ & $\begin{array}{c}\text { slope } \delta_{X_{e}} \text { vs } \\
\text { Ce concn, ppm/ } \\
\left(10^{20} \mathrm{Xe} / \mathrm{g} \text { of Na-Y) }\right.\end{array}$ \\
\hline benzene & 2 & $89.7 \pm 0.1$ & $3.8 \pm 0.1$ \\
& 1 & $75.1 \pm 1.1$ & $3.9 \pm 0.2$ \\
trimethylbenzene & 0.5 & $68.6 \pm 0.1$ & $4.0 \pm 0.1$ \\
& 2 & $89.0 \pm 1.3$ & $5.4 \pm 0.3$ \\
n-hexane & 1 & $75.9 \pm 0.4$ & $4.5 \pm 0.1$ \\
& 0.5 & $71.2 \pm 0.7$ & $3.6 \pm 0.1$ \\
none (this work) & 1 & $107.7 \pm 1.2$ & $4.4 \pm 0.3$ \\
none (ref2) & 0.5 & $86.7 \pm 0.8$ & $3.4 \pm 0.2$ \\
& & $74.7 \pm 0.5$ & $3.5 \pm 0.1$ \\
& & $58.9 \pm 0.5$ & $4.4 \pm 0.1$ \\
& & $58 \pm 4$ & $4.6 \pm 0.2$
\end{tabular}

Table 4.1: ${ }^{129} \mathrm{Xe}$ chemical slopes and intercepts.

molecules may not have a preference to be attached parallel to the surface of the supercage, but protrude into the center of the cage, or may block the channels [163], allowing for a greater interaction with xenon. This is illustrated in Figure 4.6A for the case of 2 molecules per supercage. The orientation of $n$-hexane depicted in this Figure would explain the large chemical shift values for $n$-hexane as compared with those of benzene and trimethylbenzene, even though one would expect a saturated hydrocarbon such as $n$-hexane to criate less distortion of the xenon electron cloud than benzene with its $\pi$-electron cloud. By blocking the channels or extending into the volume of the supercage, $n$-hexane is expected to have a higher probability of interaction than guest molecules attached parallel to the surface of the supercage (Figure 4.6B-D), and thus produce a larger xenon-129 chemical shift value.

We turn now to the differences in the slopes of the chemical shift values versus xenon concentration. The third column of Table 4.1 shows the slopes of the best fits (by least squares) to the data. In previous work, the slopes of the chemical shift 

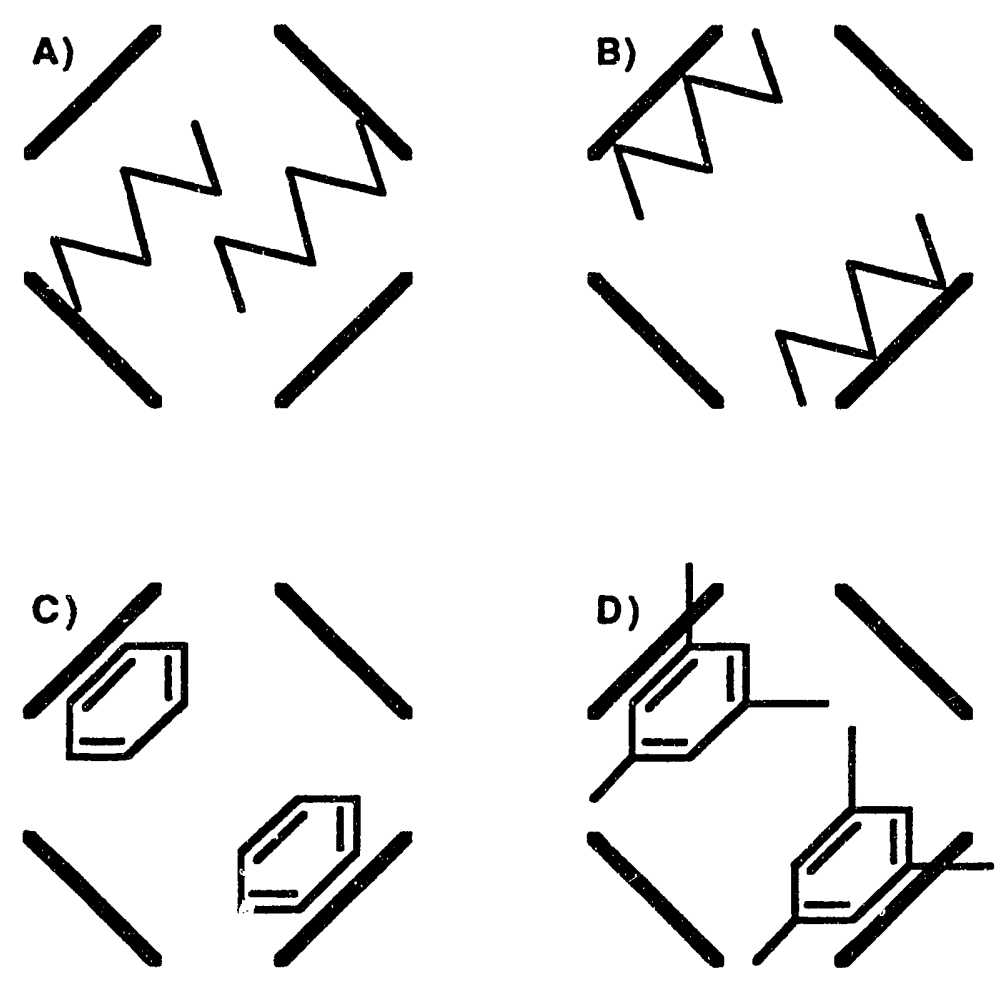

Figure 4.6: Schematic illustration of the various guest species adsorbed inside the Na-Y zeolite supercages: (A) two n-hexane moecules adsorbed normal to the supercage surface, (B) two $n$-hexane moeclues adsorbed parallel to the supercage surface, (C) two benzene molecules, and (D) two trimethylbenzene molecules. 
plots have been related either to the void volume of the supercages [96], or to channel blockage [161]. Blockage of the interconnecting channels would tend to confine the xenon atoms, increasing the xenon atom's collision frequency. Consequently, xenon's chemical shift value would increase due to the increased, time-averaged distortion of the electron cloud. Another factor which could affect the slopes is the size of the guest molecules. High concentrations of large adsorbed guests would decrease the void volume of the supercage, leading again to an increased collision frequency. In this way, both channel blockage and diminished void volume could produce a larger slope in the plot of xenon chemical shift versus adsorbed xenon concentration. Unfortunately, it is not possible to distinguish the effects of these two cases in our present experiments. We can, however, suggest a few explanations which are consistent with our results.

For the three benzene samples, the similarity of the chemical shift slopes indicates that the inclusion of benzene probably does not interfere with xenon by taking up volume in the middle of the supercages or by blocking the channels. This point agrees with previous studies which suggest that benzene is attached to the walls of the supersages $[160,162]$. The diameter of the zeolite surface near the window (cation site $\mathrm{S}_{I I}$ ) is approximately $6 \AA$, roughly the kinetic diameter of benzene (see Figure 4.6C). For the trimethylbenzene case, the situation is not so clear. If trimethylbenzene absorbs like benzene by attaching near th $: S_{I I}$ cation sites, then it would likely interfere with xenon by blocking the channels due to its larger size. The kinetic diameter of trimethylbenzene is $7.5 \AA$, so it would extend into the channels as shown in Figure 4.6D. On the other hand, if trimethylbenzene does not attach to the supercage walls, it could occupy a substantial fraction (up to $40 \%$ in the two guest molecule concentration) of the $1100 \AA^{3}$ supercage volume. An explanation of the slope data for the $n$-hexane samples is not readily apparent either. One reason for the larger 
slope seen only at a concentration of 2 hexane molecules per supercage may be that two hexanes molecules are necessary in each supercage to provide an effective net in order to interfere sufficiently with xenon as shown in Figure 4.6A. Further work is required to resolve these points.

\subsection{Conclusions}

Xenon-129 NMR has been applied to the study of molecules adsorbed in zeolites. The technique has previously been used to analyze the structure (cavity dimensions, inter-connecting pore sizes, and defects) of various zeolites, as well as to characterize exchangeable cations and metal particles. In the present case of adsorbed organic molecules, xenon-129 NMR indicates a difference in guest molecule-zeolite surface interactions. $n$-Hexane molecules are probably adsorbed normal to the surface while benzene and trimethylbenzene are adsorbed parallel to the surface. We have also found evidence for blocking of the supercage windows due to guest molecule size or orientation.

A complement to this work would be to localize the xenon inside a single supercage either by blocking the channels between the cages, or by cooling the sample. Magic-angle spinning experiments would be necessary to compensate for an increase in line width due to the lack of averaging of the anisotropic part of the xenon chemical shift as the temperature is lowered. 


\section{Chapter 5}

\section{Measurement of Xenon Clusters}

\section{in Na-A Zeolite}

The xenon in Na-A zeolite system presents an interesting opportunity to study the packing of atoms in very small spaces where the finite atomic size of the guest influences the packing arrangement. There has been a great deal of recent interest in probability functions that describe the distribution of atoms and molecules over small independent subvolumes $[76,77,164,165,166]$. These modeling efforts have significantly advanced the theoretical basis for phenomena associated with phase transitions, adsorption in small pore spaces, and configurations of molecules in bulk gases and liquids. Though the importance of local dynamics and structure to a fluid system's macroscopic behavior is well-known $[167,168,169]$, correlations with developing molecular theories and numerical simulations have proven difficult because there are few experimental data. In this chapter, experimental measurements using ${ }^{129} \mathrm{Xe}$ NMR of the distribution probabilities are made for atoms confined in small, independent subvolumes, specifically for high pressure xenon occluded in microporous Na-A zeolite cavities. 
Na-A zeolite provides an ordered matrix that can trap clusters of atoms and molecules in its void space if the molecular diameter of the guest slightly exceeds the pore diameter of the zeolite at room temperature. Assemblies of $0.44-\mathrm{nm}$ diameter xenon atoms, for example, can be introduced into the $\alpha$-cages of $\mathrm{Na}$-A zeolite through framework windows $0.42 \mathrm{~nm}$ in diameter at room temperature, by heating the zeolite to $523 \mathrm{~K}$ in the presence of xenon gas. It is possible to introduce xenon at lower pressures as well [170]. Subsequent cooling of the $\mathrm{Xe} / \mathrm{Na}-\mathrm{A}$ system to room temperature results in the confinement of xenon guest species inside the $1.14 \mathrm{~nm}$-diameter $\mathrm{Na}$-A $\alpha$-cages, as illustrated schematically in Figure 5.1. The ${ }^{129} \mathrm{Xe}$ chemical shift is sensitive to local interactions experienced by adsorbed xenon atoms and has been used to distinguish different populations of xenon in Na-A cavities [171, 172]. The distribution of occluded xenon guests within the Na-A $\alpha$-cages can be modified by varying the equilibrium xenon loading pressure at $523 \mathrm{~K}$.

\subsection{Sample Preparation}

$\mathrm{Na}-\mathrm{A}$ zeolite samples were dehydrated by heating at $673 \mathrm{~K}$ under vacuum (less than $10^{-5}$ torr) for $10 \mathrm{~h}$ in a $1-\mathrm{cm}$ diameter stainless steel, high pressure bomb [104]. After cooling the zeolite to room temperature, approximately 1-3 mmole xenon (a natural abundance mixture of isotopes, including ${ }^{129} \mathrm{Xe}$ and ${ }^{131} \mathrm{Xe}$ ) was introduced to the bomb at $298 \mathrm{~K}$ using a standard vacuum rack apparatus. High xenon loading pressures were produced by first condensing the gas in the bomb assembly (immersed in a liquid-nitrogen bath), isolating the bomb from the vacuum rack, and then reexpanding the xenon gas into the $10-\mathrm{cm}^{3}$ bomb volume. The zeolite samples in the bomb were then heated to $523 \mathrm{~K}$ for $4 \mathrm{~h}$ ( $8 \mathrm{~h}$ for the sample loaded at $210 \mathrm{~atm}$ ), permitting xenon atoms to penetrate the expanded Na-A $\alpha$-cage structure. Xenon 


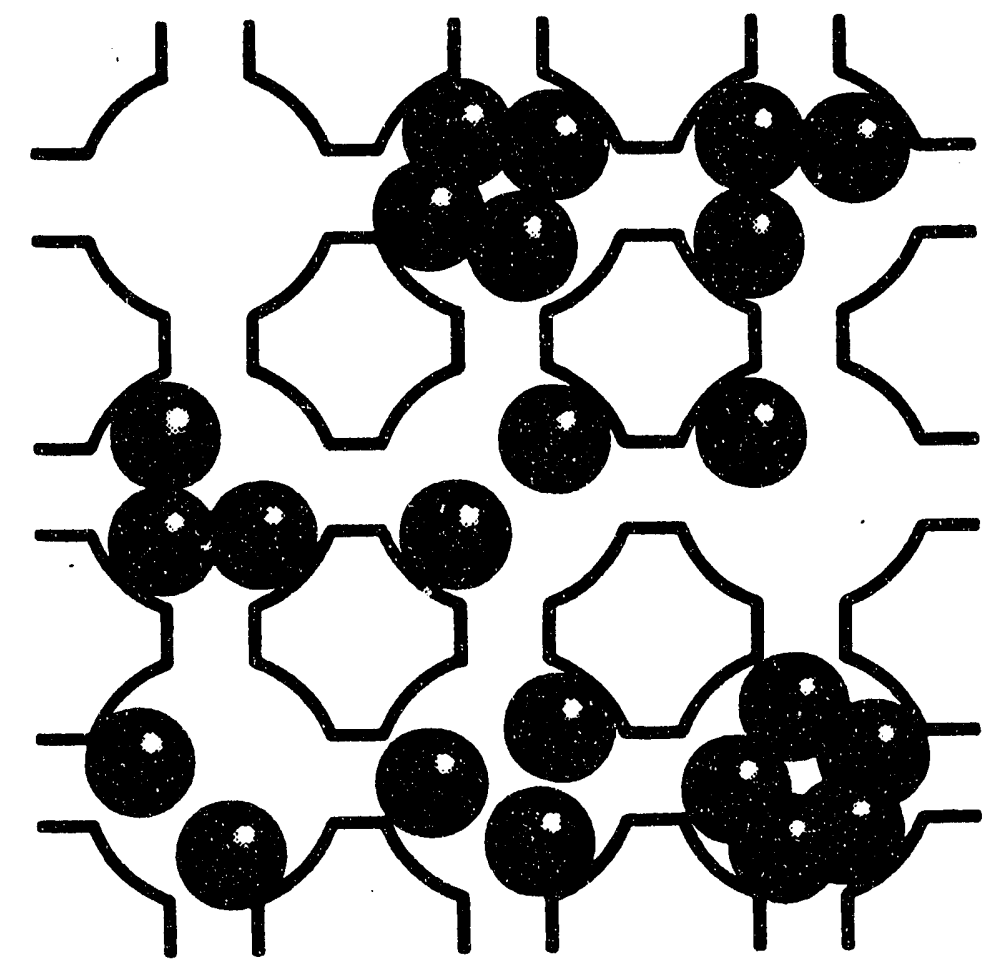

Figure 5.1: Schematic diagram of Na-A zeolite $\alpha$-cages containing different populations of occluded xenon guest atoms (represented by the shaded spheres). 
loading pressures of up to 210 atm at $523 \mathrm{~K}$ were achieved under conditions of adsorption equilibrium. The samples were subsequently quenched by immersion of the bomb in an ice-water bath, trapping the xenon atoms in the $\mathrm{Na}-\mathrm{A} \alpha$-cages. Quenching rates were varied from about $0.02-2 \mathrm{~K} / \mathrm{s}$ with no detectable effect on the xenon distribution in samples otherwise treated identically.

After quenching, the $\mathrm{Xe} / \mathrm{Na}-\mathrm{A}$ samples were transferred to glass ampoules in a glove box under dry nitrogen to prevent contamination by atmospheric moisture. The sample-containing ampoules were then evacuated, charged with 100 torr of dry oxygen gas, and finally sealed. The addition of a small quantity of paramagnetic oxygen reduced the spin-lattice relaxation time $\left(T_{1}\right)$ of ${ }^{129} \mathrm{Xe}$ in these samples, allowing use of shorter delays (typically $1 \mathrm{~s}$ ) between $90^{\circ}$ pulses. Xenc $n-129$ NMR spectra were obtained after signal averaging 5000 to 10,000 scans on a spectrometer operating at $110 \mathrm{MHz}$. Chemical shifts are referenced to xenon gas at very low pressure [2] and are accurate to $\pm 0.5 \mathrm{ppm}$.

\subsection{Results}

Figure 5.2 shows room-temperature ${ }^{129} \mathrm{Xe}$ NMR spectra of dehydrated Na-A zeolite samples containing occluded xenon atoms adsorbed under different equilibrium loading pressures at $523 \mathrm{~K}$. The ${ }^{129} \mathrm{Xe}$ nuclei become less shielded with increasing guest density [11], so that multiple peaks are observed from a single $\mathrm{Xe} / \mathrm{Na}-\mathrm{A}$ sample. Xenon atoms in $\mathrm{Na}-\mathrm{A} \alpha$-cages with different guest occupancies have characteristic NMR frequencies that permit the relative populations of $\alpha$-cages containing different numbers of xenon guests to be determined [73]. Each peak corresponds to a ${ }^{129} \mathrm{Xe}$ signal from $\alpha$-cages containing a different number of occluded xenon atoms. Small variations in the chemical shift of ${ }^{129} \mathrm{Xe}$ in cavities containing identical xenon 

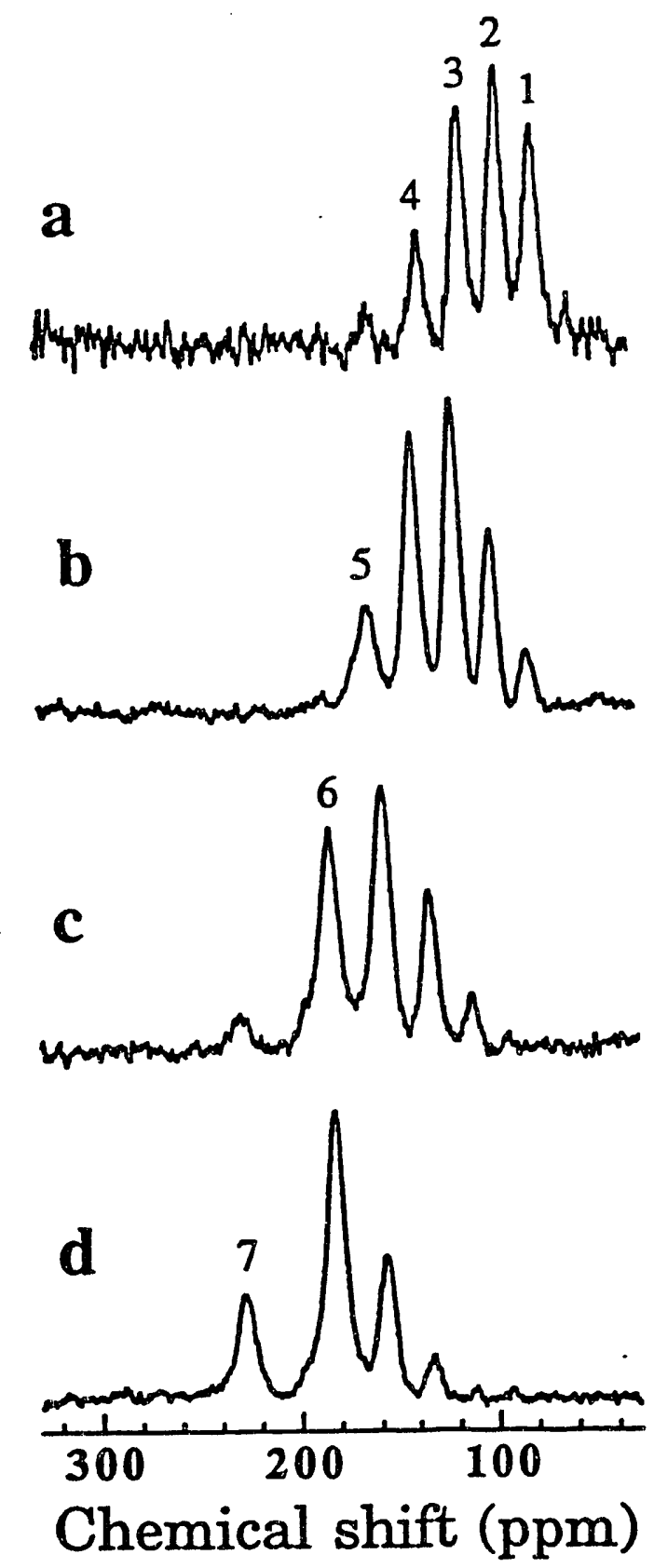

Figure 5.2: Room-temperature ${ }^{129} \mathrm{Xe}$ NMR spectra of xenon occluded in NaA zeolite at $523 \mathrm{~K}$ at different equilibrium loading pressures: (a) $8 \mathrm{~atm}$, (b) $40 \mathrm{~atm}$, (c) $150 \mathrm{~atm}$, and (d) $210 \mathrm{~atm}$. Each peak corresponds to a ${ }^{129} \mathrm{Xe}$ signal from xenon in $\alpha$-cages containing different numbers of occluded Xe atoms (indicated by the numbers above the respective peaks). 
populations are most likely due to small amounts of co-adsorbed water [173]. The distribution of xenon in these different cage environments can be determined from the relative areas of the peaks in the ${ }^{129} \mathrm{Xe}$ NMR spectrum, after normalizing the peak areas by the occupation numbers [74]. As illustrated in Figure 5.2, xenon occupancy of the Na-A $\alpha$-cages is a sensitive function of the xenon loading pressure at equilibrium. The data show a monotonic increase in the mean xenon population $\bar{n}$ as the loading pressure is increased, with xenon occupancy of the $\alpha$-cages approaching an apparent loading limit of about $7 \mathrm{Xe} / \alpha$-cage, although this is probably not the absolute limit for the zeolite.

\subsection{Discussion}

At low xenon loadings, where the atomic volume of the xenon is negligible, the distribution of atoms should be well described by binomial statistics. For a loading of $N_{0}$ xenon atoms in an ensemble volume $V_{o}$, the probability of finding an $\alpha$-cage with $n$ xenon guests can be represented by a binomial distribution:

$$
f_{N_{o}, p}(n)=\left(\begin{array}{c}
N_{o} \\
n
\end{array}\right) p^{n}(1-p)^{N_{o}-n}
$$

where $f_{N_{o}, p}(n)$ is the normalized number of $\mathrm{Na}$-A $\alpha$-cages in an ensemble volume $V_{o}$ possessing $n$ xenon guests, and $p=V / V_{0}=\bar{n} / N_{0}$ is the probability that a xenon atom will occupy a given $\alpha$-cage with subvolume $V$. As observed by Rowlinson and Woods [77], the probability $p$ is not constant, but varies as a weak function of xenon occupancy according to the degree of subvolume inciependence. The xenon populations $f(n)$ are measured experimentally for xenon atoms occluded in Na-A $\alpha$-cages (for $n>0$ ) by integrating the peaks in the ${ }^{129} \mathrm{Xe}$ NMR spectra of Figure 5.2, and dividing by the respective occupancies [74]. 
As illustrated in Figure 5.3(a) for Xe/ Na-A systems with mean populations $\bar{n}$ of less than about $3 \mathrm{Xe}$ atoms per $\alpha$-cage, agreement is excellent between the experimentally measured xenon occupancies $f(n)$ and those values predicted by binomial statistics. As the loading pressure is increased, the finite sizes of the guest atoms play an important role in establishing the xenon occupancies observed in Figures 5.3(b-d). Güémez and Velasco [76] have suggested that the distribution of atoms with finite volumes (or equivalently, among mutually exclusive lattice sites) should display a hypergeometric dependence of the form [75]:

$$
f_{N_{o}, p}(n)=\frac{\left(\begin{array}{c}
N_{0} \\
n
\end{array}\right)\left(\begin{array}{c}
M-N_{o} \\
K-n
\end{array}\right)}{\left(\begin{array}{c}
M \\
K
\end{array}\right)},
$$

where $M=V_{o} / \sigma$ is the total number of lattice sites available in the ensemble volume $V_{o}, K=V / \sigma=p M$ is the number of lattice sites available in a single $\alpha$-cage with subvolume $V, \sigma$ is an effective molecular volume, and $N_{o}=\bar{n} / p$ is the ensemble xenon population as before. After substituting for $M$ and $N_{0}$, equation (5.2) becomes:

$$
f_{N_{0}, p}(n)=\frac{\left(\begin{array}{c}
\bar{n} / p \\
n
\end{array}\right)\left(\begin{array}{c}
K / p-\bar{n} / p \\
K-n
\end{array}\right)}{\left(\begin{array}{c}
K / p \\
K
\end{array}\right)}
$$

with $\bar{n}$ and $K$ representing adjustable parameters.

The data to be fit consist of the different peak areas divided by the occupancies and normalized so that their total is one. A program written in Turbo Pascal fits the 


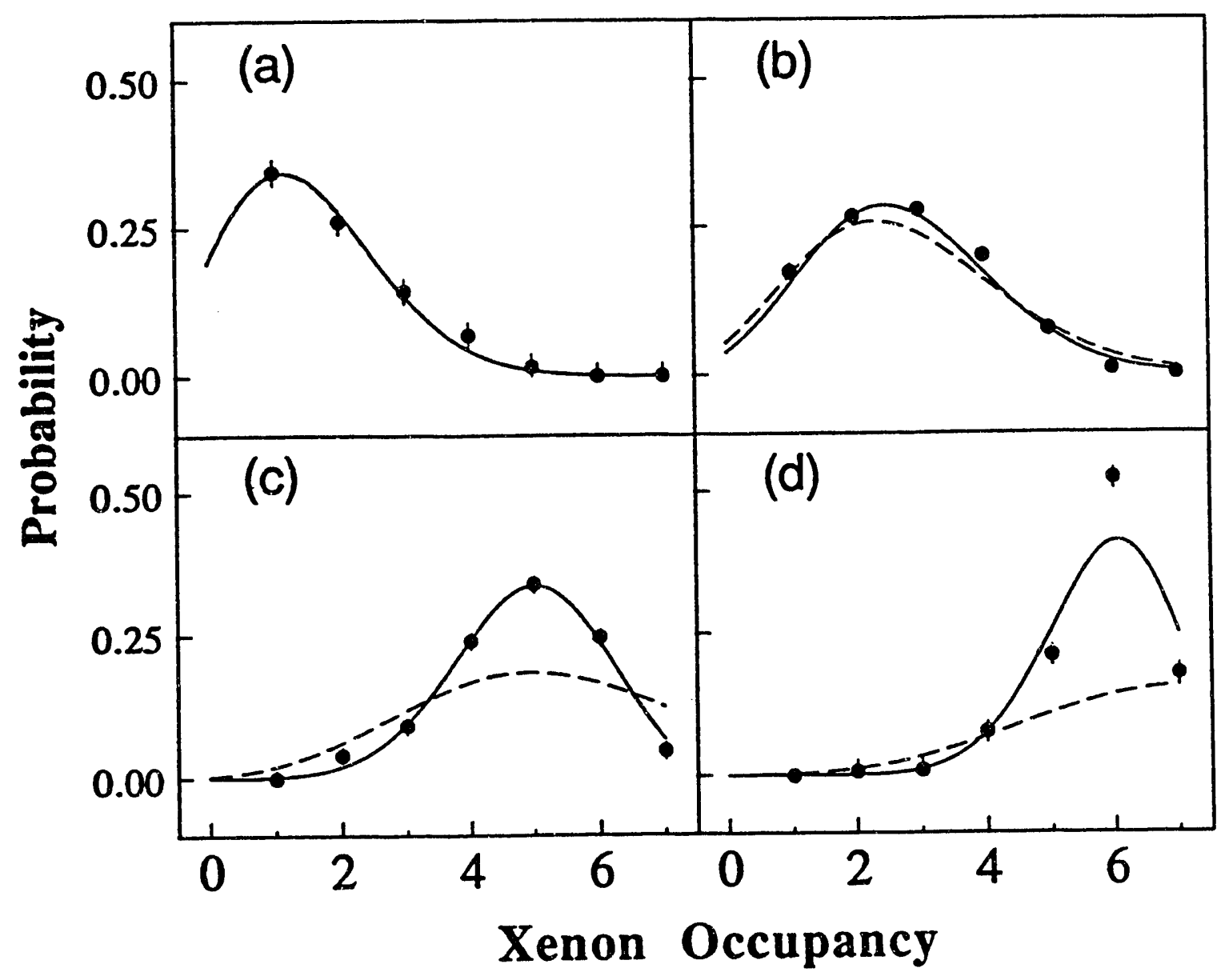

Figure 5.3: Plots of normalized experimental ${ }^{129} \mathrm{Xe}$ NMR peak areas, $f(n)$, ( $(\boldsymbol{)})$ versus the number of xenon atoms occluded in an ensemble of $\mathrm{Na}-\mathrm{A} \alpha$-cages at different loading pressures: (a) $8 \mathrm{~atm}$, (b) $40 \mathrm{~atm}$, (c) $150 \mathrm{~atm}$, and (d) $210 \mathrm{~atm}$. Accompanying the data are binomial (-) and hypergeometric (- . - ) best fits to the data computed using equations (5.1) and (5.3) 
data to equations (5.1) and (5.3) to a least squared errors criterion using a "Simplex" searching routine [174]. Renormalization is necessary when there is a finite probability of zero occupancy, since NMR is incapable of detecting zero xenon-129 atoms. As shown in Figure 5.3(a), for low xenon loadings $(\bar{n}<3 \mathrm{Xe} / \alpha$-cage), the binomial and hypergeometric fits to the ${ }^{129} \mathrm{Xe}$ NMR data are nearly coincident. For mean xenon populations $\bar{n}$ above about $3 \mathrm{Xe} / \alpha$-cage [Figs. $5.3(\mathrm{~b}-\mathrm{c})$ ], the hypergeometric fits obtained using equation 5.3 with $K=7$ agree well with the ${ }^{129} \mathrm{Xe}$ NMR data, consistent with the maximum number of xenon atoms observed in the spectrum of Figure 5.2(d).

At the highest xenon loading studied $(\bar{n}=5.9)$, the observed xenon distributions are consistently narrower than both the hypergeometric and binomial predictions [Fig. 5.3(d)]. This may arise from non equilibrium conditions produced by hindered diffusion of xenon atoms through $\mathrm{Na}-\mathrm{A}$ cavities crowded with other xenon guests. However, a narrow xenon distribution is also the result anticipated (from Monte Carlo simulations, for example) for real fluids, with continuously moving molecules, that present a less accommodating configuration for placement of a molecular guest in an arbitrary subvolume [77]. Moreover, the geomtiric constraints imposed by the $\alpha$-cages on guest packing configurations are undoubtedly appreciable, especially at high guest loadings. Separate theoretical studies $[176,177,178]$ and adsorption experiments $[173,179]$ indicate that xenon atoms have a strong affinity for aluminosilicate surfaces and consequently maintain close proximity to the $\alpha$-cage walls as a highly mobile, adsorbed phase at room temperature. The result is that xenon packing arrangements are almost certainly dominated by local surface effects and 6eometric constraints even at low guest loadings. Based on an idealized geometric model, a pentagonal bipyramidal arrangement of xenon atoms tangent to the interior walls of the $\alpha$-cages appears feasible near the saturation occupancy when $n=7$ 
xenon atoms per cavity. Recent Monte Carlo simulations have indeed demonstrated the likelihood of such xenon polyhedra in $\mathrm{Na}-\mathrm{A}$ [180]: assemblies of six xenon atoms are expected to possess cubic symmetry, while a lower symmetry configuration is anticipated when $n=7$. Theoretical prediction of the maximum number of xenon guests capable of being accommodated inside a $\mathrm{Na}-\mathrm{A} \alpha$-cage remains an open and challenging problem.

\subsection{Conclusions}

These are the first experimental results that measure atomic distribution statistics for different guest loadings in a macroscopic ensemble. These data clearly demonstrate the role of finite atomic volume on occupancies in small subvolumes. The better fit of the data to a hypergeometric, rather than a binomial, distribution indicates that atoms in the cavities of the zeolite behave as subvolumes of a finite fluid and not as subvolumes in independent equilibrium with an infinite reservoir. Local structural information provided by microscopic distribution probabilities has important consequences for the prediction of bulk and confinement-modified fluid properties, particularly as they relate to transitions between phases or to adsorption and transport in microporous materials. 


\section{Chapter 6}

\section{Optically-Pumped Xenon}

\section{Experiments in Low Magnetic}

\section{Field}

In this chapter experiments are described that use optical pumping and opticallydetected NMR to enhance the signal from low pressure gas samples of ${ }^{129} \mathrm{Xe}$ and ${ }^{131} \mathrm{Xe}$. With xenon's relatively low sensitivity and very long relaxation times (up to 30 minutes for ${ }^{129} \mathrm{Xe}$ ), it would otherwise be difficult to detect the approximately $10^{16}$ to $10^{17}$ xenon atoms used in these experiments. However, by combining the optical pumping methods described in Chapter 1 to increase the nuclear polarization signal, with optical detection methods to increase the signal detection efficiency, sufficiently high sensitivity may be achieved. Following the pioneering work of Happer and coworkers at Princeton [84], we have conducted optical pumping experiments on ${ }^{129} \mathrm{Xe}$ in order to understand the mechanics of optical pumping and to maximize the relevant parameters such as relaxation times, pumping rates, polarizations, for use in further experiments. ${ }^{129} \mathrm{Xe}$ has been shown to be relatively easily polarized via 
spin exchange collisions with optically-pumped rubidium atoms [32] due to its large electron cloud polarizability and long relaxation times. The optical pumping of ${ }^{129} \mathrm{Xe}_{\mathrm{e}}$ has been investigated using optical detection methods to observe longitudinal $\left(T_{1}\right)$ decay times as a function of the cell temperature and the cell surface. An example of the high sensitivity of the optical pumping and optical detection is demonstrated in Figure 6.1. The ${ }^{129} \mathrm{Xe}$ longitudinal decay lasts more than 20 minutes at $60^{\circ} \mathrm{C}$.

The optical pumping and detection methods have also been used to probe interactions of quadrupolar ${ }^{131} \mathrm{Xe}$ at the glass surface of the pumping cells. When ${ }^{131} \mathrm{Xe}$ is placed in asymmetric pumping cells, a residual quadrupolar interaction exists that is due to interactions of quadrupolar xenon nucleus with electric field gradients at the surface of the cell walls that causes a splitting in the NMR frequencies of ${ }^{131} \mathrm{Xe}[83,84,181]$. Happer and coworkers $[84,181]$ have investigated this interesting effect, observing splittings of up to $300 \mathrm{mHz}$ in the ${ }^{131} \mathrm{Xe}$ NMR. Similar methods have been used in initial observations of ${ }^{131} \mathrm{Xe}$ quadrupolar splittings [83] as well as in observations of very long noble gas decays used to probe for spatial anisotropies [182]. These experiments rely on carefully shielded and stabilized low magnetic fields of about 0.1 gauss in order to reduce, as much as possible, additional dephasing in the decay of the observed magnetization due to residual magnetic field inhomogeneities. Work in the Pines laboratory has concentrated on extending previous methods in order to improve resolution or increase the bandwidth of these types of experiments. Drawing on the enormous literature of compensated radio frequency pulse sequences ([183] and references within), several sequences have been applied with an eye towards circumventing the magnetic field stabilization techniques and shielding necessary in earlier studies. Also, these improvements will allow one to increase the bandwidth of these experiments to investigate surfaces that might have larger interactions with ${ }^{131} \mathrm{Xe}$. 


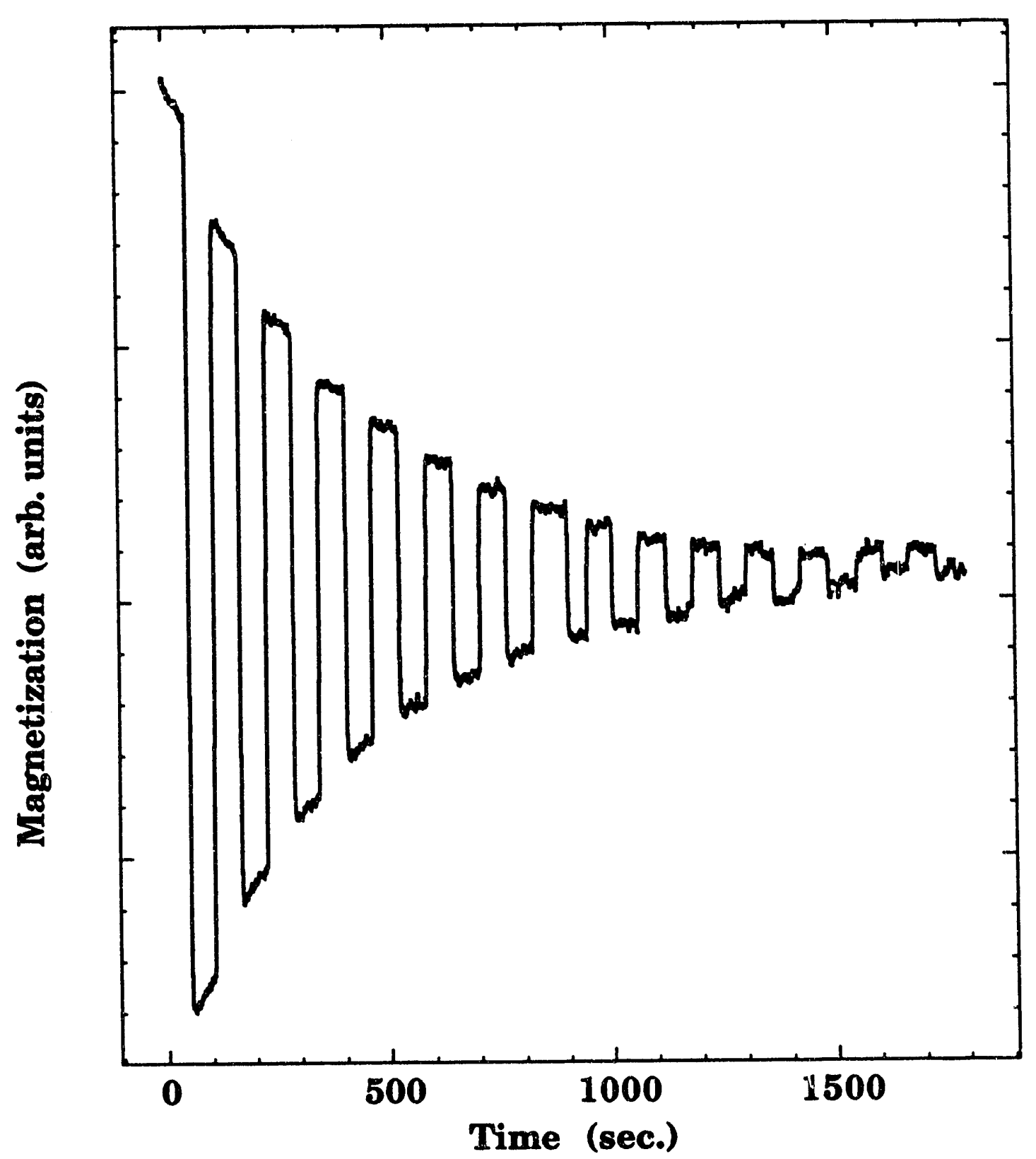

Figure 6.1: ${ }^{129} \mathrm{Xe}$ optically-dectected longitudinal decay. Sensitivity to drifts in the detected light are reduced by reversing the magnetization every minute using adiabatic rapid passage. 


\subsection{Theory}

\subsubsection{Optical Pumping and Relaxation}

A general theory of optical pumping experiments was presented in Chapter 1. Briefly, the polarization rate of xenon is determined by the laser power and the rubidium density:

$$
\frac{1}{T_{\text {pump }}}=\frac{\Gamma}{2}[R b] \bar{v} \sigma_{x e}
$$

where $\Gamma$ is the laser pumping rate, $[R b]$ is the rubidium density, $\bar{v}$ is the relative velocity of xenon and rubidium and $\sigma_{x e}$ is the xenon collision cross section. [Rb] depends on the temperature such that [184]:

$$
[R b]=10^{23.97-4132 / T},
$$

with $T$ in Kelvin. At $65^{\circ} \mathrm{C}[\mathrm{Rb}] \approx 10^{12}$ atom $/ \mathrm{cm}^{3}$.

Once the pumping light is turned off, the relaxation of xenon is caused primarily by spin-exchange collisions with rubidium. The xenon nucleus couples to the rubidium $5 s$ electron which relaxes much more rapidly during collisions with the cell walls. Happer has investigated the rubidium density-dependent relaxation of ${ }^{129} \mathrm{Xe}$, and found that the relaxation rate may be written:

$$
\frac{1}{T_{1}}=[R b] C+\gamma_{\text {wall }}
$$

where $\mathrm{C}$ is a constant. At temperatures above $100^{\circ} \mathrm{C}$, the $T_{1}$ is reduced to about 5 minutes. Collisions with the glass walls, which contain paramagnetic impurities, are important at lower temperatures. At temperatures below about $45^{\circ} \mathrm{C}$, where $\gamma_{\text {wall }}$ dominates, the relaxation time can approach 30 minutes or more in coated cells (see Chapter 1). 
Volk et al. [86] have found that the spin-exchange cross section for ${ }^{131} \mathrm{Xe}$ is much lower than for ${ }^{129} \mathrm{Xe}$ and therefore rubidium does not affect the longitudinal relaxation until one goes to higher temperatures. The authors have investigated $\mathrm{T}_{1}$ relaxation of ${ }^{131} \mathrm{Xe}$ in spherical cells which are not dominated by quadrupolar relaxation. They found that the relaration is due to three effects, wall collisions at low temperatures, spin-exchange to rubidium at high temperatures, and a third, temperature independent term:

$$
T_{2}^{-1}=[R b] \sigma_{e x} \bar{\nu}+\Gamma e^{2 E / k T}+\left(T_{2}^{\prime}\right)^{-1}
$$

Two other relaxation mechanisms are apparent in the optical pumping experiments. First, diffusion of the gas through residual magnetic field inhomogeneities (in either static $B_{0}$ or $\operatorname{rf} B_{1}$ fields) causes longitudinal relaxation. This effect was investigated by two groups and found to be [185]:

$$
\frac{1}{\Gamma_{1}}=-\gamma^{2} D \frac{B_{x}^{2}+B_{y}^{2}}{B_{\circ}^{2}}
$$

where $B_{x}$ is the field gradient in the $x$ direction. Notice that the relaxation is not affected by gradients in the $z$ direction. Relaxation of this type is expected to be small in our experiments for $B_{z} \gg B_{x}, B_{y}$. Secondly, as discovered by Hahn [186] (and later studied by Carr and Purcell [225]), transverse magnetization dephases rapidly due to diffusion in an inhomogeneous magnetic field:

$$
M_{\text {trans }}(t)=M_{\circ} e^{-t / T_{2}} e^{-\frac{2}{3}\left(\gamma B_{z}\right)^{2} D / t^{3}}
$$

This dephasing mechanism was dominant in our experiments, and several methods were tried to circumvent this problem. 


\subsubsection{Quadrupolar Interactions with the Wall}

\section{Energy Splittings}

In the pumping cells, ${ }^{131} \mathrm{Xe}$ gas atoms are primarily subject to a Hamiltonian which contains two parts:

$$
H=H_{Z}+H_{Q}
$$

where $H_{Z}=-\gamma \hbar H_{\circ} I_{z}$ is the Zeeman interaction for xenon in the gas phase, with $\omega=\gamma H_{\circ}$, and $H_{Q}$, the quadrupolar interaction, is given by:

$$
H_{Q}=\frac{e^{2} q Q}{4 I(2 I-1)}\left(3 I_{z^{\prime}}^{2}-I(I+1)\right)+\frac{1}{2} \eta\left(I_{+}^{2}+I_{-}^{2}\right) .
$$

The quadrupolar interaction arises from the interaction of the quadrupolar ${ }^{131} \mathrm{Xe}$ nucleus with the electric field gradients at the surface of the optical cells. The asymmetry parameter, $\eta$ is defined as:

$$
\eta=\left(V_{x^{\prime} x^{\prime}}-V_{y^{\prime} y^{\prime}}\right) / V_{z^{\prime} z^{\prime}}
$$

where $V_{z^{\prime} z^{\prime}}$ is the electric field gradient in the quadrupolar axis of quantization, $z^{\prime}$, which may not coincide with the laboratory $z$ axis. In the optical pumping experiments, the electric field gradient is assumed to be axially symmetric and point perpendicular to the surface, therefore $\eta=0$. The quadrupolar interaction frame can be rotated to the laboratory frame, [13] by substituting:

$$
I_{z^{\prime}}=I_{z} \cos \theta+I_{x} \sin \theta
$$

in equation (6.8). Due to the short adsorption time of xenon at the surface, the quadrupolar interaction is small, so that $H_{Q} \ll H_{z}$, and to first order, we consider only the secular part of the quadrupolar Hamiltonian and may write:

$$
H_{Q}=\frac{e^{2} q Q}{4 I(2 I-1)}\left(3 I_{z}^{2}-I^{2}\right) \frac{1}{2}\left(3 \cos ^{2} \theta-1\right) .
$$


The energy levels of the total Hamiltonian [equation (6.7)] are given by:

$$
E=-m_{z} \omega \hbar+\frac{e^{2} q Q}{4 I(2 I-1)}\left[3 m^{2}-I(I+1)\right]\left(3 \cos ^{2} \theta-1\right)
$$

The effect of this quadrupolar perturbation on the energy levels of the larger Zeeman Hamiltonian may be seen in Figure 6.2. The unperturbed Zeeman levels are equally spaced by the Larmor frequency of ${ }^{131} \mathrm{Xe}$, or about $17 \mathrm{kHz}$ in our 49 gauss field. In an asymmetric cell,Figure 6.2a, a residual quadrupolar interaction exists, even after averaging over the entire surface of the cell. The perturbation can be seen in the energy level diagram shown in Figure $6.2 \mathrm{~b}$ that results in a splitting of the previously degenerate transition frequencies such that there are now three different resonances, the carrier at $\omega$ plus two sidebands at $\omega \pm \omega_{Q} / 2$. The quadrupolar splitting $\omega_{Q}$ is defined as the difference between the sideband frequencies.

In a nutation experiment, (where the radio frequency field is applied continuously, and usually on resonance), the signal is observed at the nutation frequency, $\omega_{1}$, which can be obtained by transforming equation (6.11) to the rotating frame $[13,140]$. The result is that the quadrupolar interaction is modified by a factor $\left(3 \cos ^{2} \beta-1\right) / 2$ where $\beta$ is the angle between the axis of rotation and the laboratory $z$ axis. For the on resonance case, $\beta=\pi / 2$, and $H_{Q}$ is multiplied by a factor of $1 / 2$.

At the surface of the cell, the ${ }^{131} \mathrm{Xe}$ nucleus is perturbed by electric field gradients, whose exact origin is unknown. It is possible that these gradients are caused by the presence of unpaired electrons at the surface (due to paramagnetic impurities, for instance), or due to the distortion of xenon's electron cloud itself, or more likely, a combination of both. It is well known that electron cloud distortions can enhance the electric field gradients felt by the nucleus, and is called a Sternheimer anti-shielding effect [188] where:

$$
V_{z z}=V_{z z}^{\circ}[1-\gamma(r)]
$$



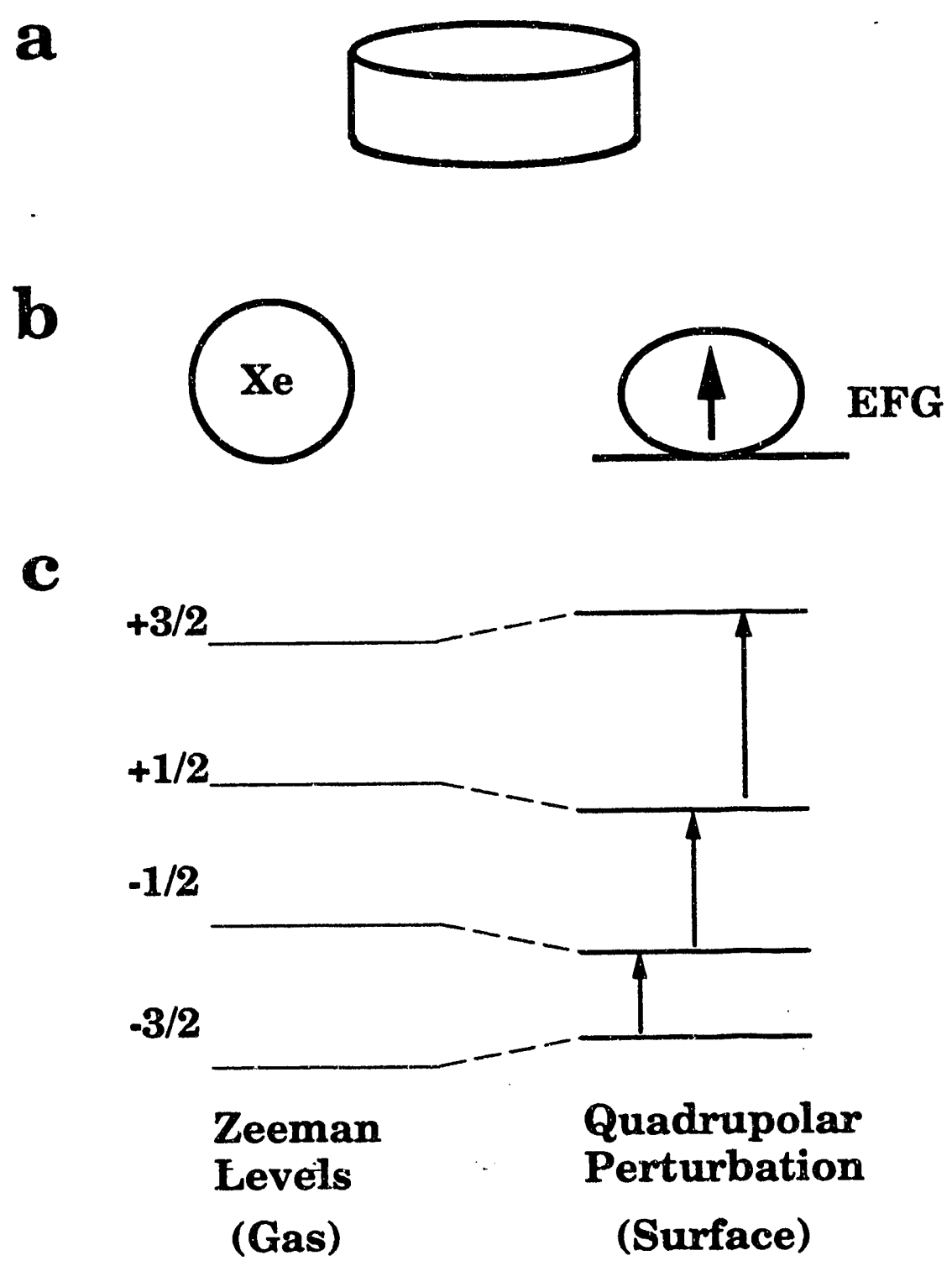

Figure 6.2: (a) Asymmetric optical pumping cell with diameter larger than height.

(b) Xenon in the gas phase and on the surface (with quadrupolar interaction). (c) ${ }^{131} \mathrm{Xe}$ energy level diagram for the quadrupolar-perturbed Zeeman Hamiltonian in asymmetric pumping cells. The three transition frequencies become non-degenerate. 
$\gamma(r)$ is 99 for $\mathrm{Cs}^{+}$and presumably larger for xenon.

\section{Surface Quadrupolar Relaxation}

A relatively full theoretical treatment of the dephasing of quadrupolar nuclei due to surface collisions was discussed by Cohen-Tannoudji [31] in his experiments on Hg relaxation. It is interesting to note that he did not observe splittings due to any asymmetry of his pumping cells. Splittings were observed much more recently in mercury pumping cells by Fortson and coworkers [189]. Nonetheless, CohenTannoudji's theory of wall relaxation is generally in accordance with experiments, with recent additions by Happer. I will briefly discuss the salient points of the theory.

The relaxation due to a time dependent perturbation is given by Abragam [15]:

$$
\frac{\partial \rho^{*}}{\partial t}=-\int_{0}^{t} \overline{\left[H_{Q}^{*}(t),\left[H_{Q}^{*}(t+\tau), \rho^{*}(t)\right]\right]} d \tau
$$

where:

$$
H_{Q}^{*}(t)=e^{i H_{Z} t} H_{Q} e^{-i H_{Z} t}
$$

is in the interaction picture [190], and similarly for $\rho^{*}$. Here, $H_{Q}^{*}(t)$ is the random perturbation, and it is assumed to have an exponential correlation-time dependence such that:

$$
\overline{H_{Q}^{*}(t) H_{Q}^{*}(t+\tau)}=\overline{H_{Q}^{2}} e^{-t / \tau_{c}}
$$

Where $H_{Q}$ is now time independent. Cohen-Tannoudji assumed that the correlation time was long compared to the sticking time, allowing him to express the overall damping rate as:

$$
T_{Q}^{-1}=\frac{\tau_{c} \tau_{s}}{\tau_{s}+\tau_{v}} \overline{\left[e^{2} q Q\right]^{2}}
$$

by carrying out the integral in equation (6.14). The ratio of the surfare sticking time to the total time, $\tau_{s} /\left(\tau_{s}+\tau_{v}\right)$ is the probability of finding the xenon at the wall. 
$\tau_{v}=V / 4 S v$ is the time between collisions and is inversely related to the surface to volume ratio and the atomic velocity, $v$.

An alternate derivation was recently provided by Happer [88] where he made no assumption about the correlation time. The final integration is made over the adsorption tirne of the xenon at the surface weighted by an exponential sticking probability:

$$
\overline{H_{Q}^{2}} \int_{0}^{\infty} \frac{d \tau}{\tau_{s}} e^{-\tau / \tau_{s}} \int_{0}^{\tau} d t \int_{0}^{t} d t^{\prime} e^{-\left|t-t^{\prime}\right| / \tau_{c}}
$$

leading to Happer's result for the quadrupolar dephasing [84]:

$$
T_{Q}^{-1}=\frac{v S}{10 V} \frac{\tau_{c} \tau_{s}^{2}}{\tau_{c}+\tau_{s}} \overline{\left[e^{2} q Q\right]^{2}}
$$

which reduces to the same result as equation 6.17 for $\tau_{c} \ll \tau_{s}$, since $4 v S / V=1 /\left(\tau_{s}+\right.$ $\left.\tau_{V}\right)$. Happer's case represents the situation where the xenon nucleus experiences the same electric field gradient during many sticking collisions with the cell walls. The $\tau_{c} \gg \tau_{s}$ case is relevant when xenon experiences a different electric field gradient for each adsorption, or when the gradient changes during the adsorption process.

\subsection{Experimental}

\subsubsection{Cell Preparation}

Cells are constructed using circular Pyrex plates $1 \mathrm{~mm}$ thick and $50 \mathrm{~mm}$ in diameter that are attached to a tube with a stopcock with $1 / 4$ " connection to a Cajun fitting on a gas rack, as shown in Figure 6.3. Happer et al. [32] describes methods for mass production of cells, but since our required numbers of cells were small, they were prepared individually.

Briefly, the cells were washed with distilled water and acetone and dried overnight at $90{ }^{\circ} \mathrm{C}$ in air. In the case of coated cells, such as for the ${ }^{129} \mathrm{Xe}$ experiments, cells 


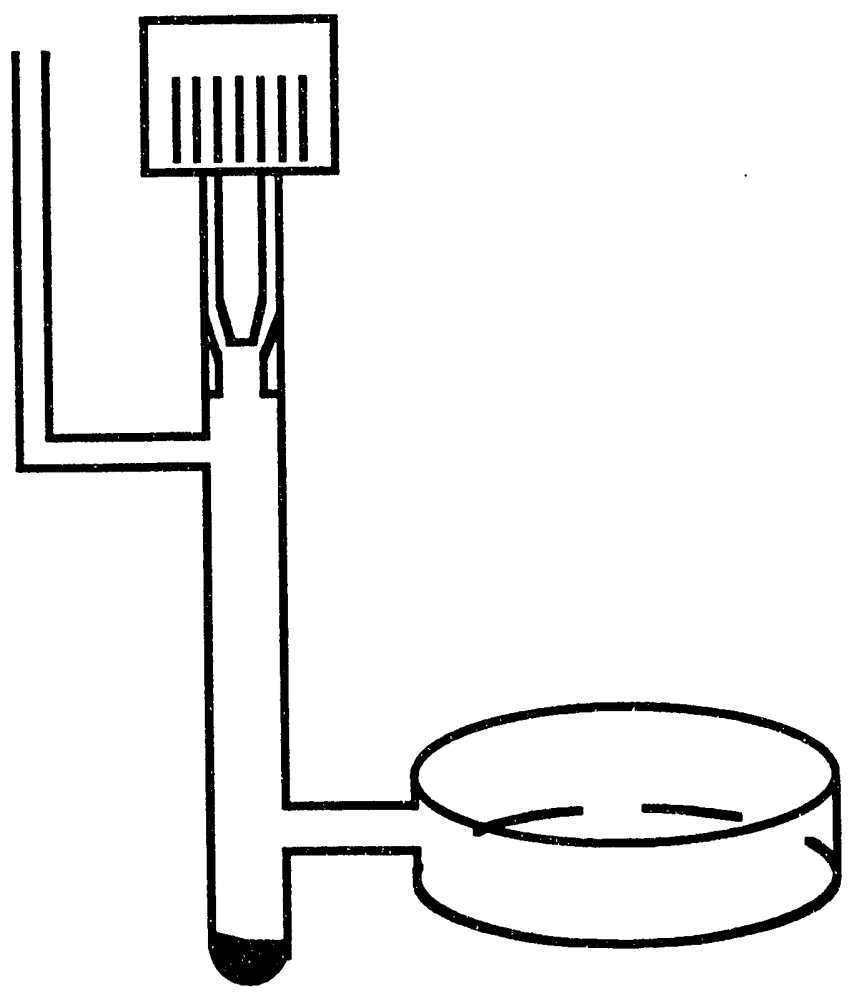

Figure 6.3: Optical pumping cell and stopcock tube with rubidium metal at the bottom. 
were rinsed with cyclohexane, then washed with a $10 \%$ solution of Surfrasil [85] in cyclohexane and finally rinsed with pure cyclohexane to get rid of any unreacted Surfrasil. Cells were then attached to a stopcock tube as shown in Figure 6.3 and attached to a gas rack.

The cells were then evacuated under vacuum $\left(10^{-5}\right.$ torr at $120^{\circ} \mathrm{C}$ overnight). Rubidium metal was placed in the bottom of the stopcock tube in a nitrogen-filled glovebox and the manifold was remounted on the gas rack. Cells were filled with a few torr of enriched ${ }^{129} \mathrm{Xe}$ or ${ }^{131} \mathrm{Xe}$ which was then frozen onto the bottom of the stopcock tube, during which time either nitrogen or a $10 \%$ hydrogen in nitrogen buffer gas was added. After waiting for the gases to mix [32], the rubidium was distilled over to the cell using a gas torch. The cells were then carefully torched off and cured at $120-150^{\circ} \mathrm{C}$ for a week or more. The curing process has been shown to be important in ${ }^{131} \mathrm{Xe}$ cells [181], and often we did not see quadrupolar splittings, or even rubidium absorption in uncured cells.

\subsubsection{Apparatus}

A schematic drawing of the experimental apparatus is shown in Figure 6.4. Pyrex glass sample cells are contained in a plexiglass oven which is heated using hot, flowing nitrogen gas, and controlled to better than $0.1^{\circ} \mathrm{C}$. A large solenoid magnet of an overwound design [191] $100 \mathrm{~cm}$ long and $30 \mathrm{~cm}$ in diameter produced a field of 50 gauss and is driven by a home-built $5 \mathrm{amp}$ constant-current power supply with $10 \mathrm{ppm} /{ }^{\circ} \mathrm{C}$ stability. The magnet also has three correction coils to reduce field gradients so that the magnet is homogeneous to better than $50 \mathrm{ppm}$ over the $100 \mathrm{~cm}^{3}$ sample volume. The field homogeneity is corrected using the NMR signal from a water sample using standard pulsed-NMR detection circuitry at $209 \mathrm{kHz}$.

The sample cell is optically pumped using circularly polarized light from a 40 mil- 
$\mathbf{a}$

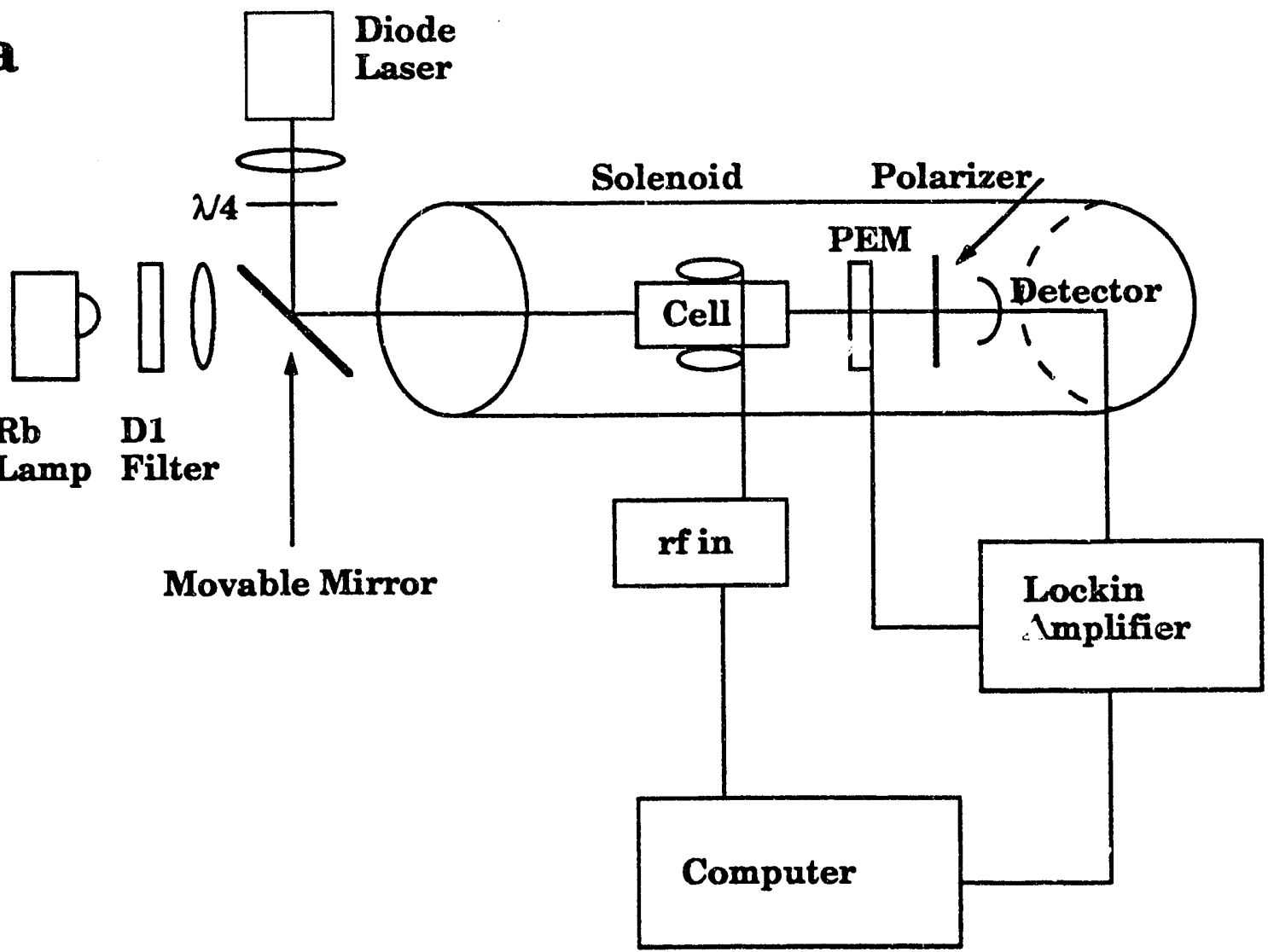

b

Pump Laser

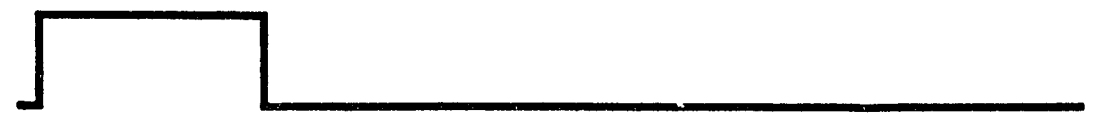

Detection Lamp

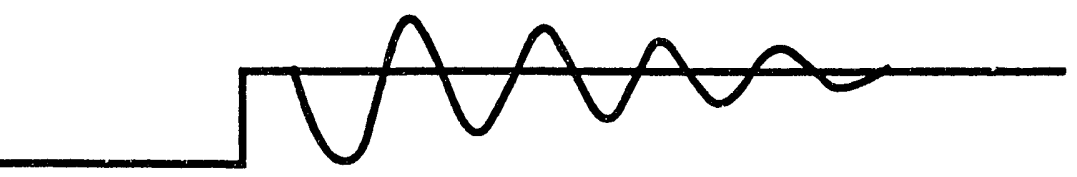

RF

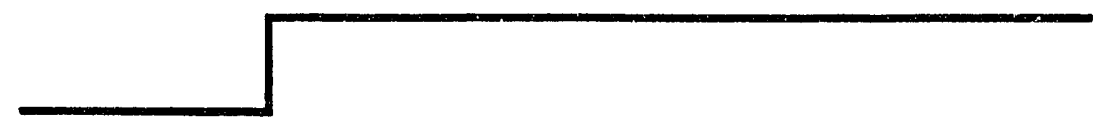

Figure 6.4: Low field optical pumping and optical detection apparatus (a) and timing diagram (b) as described in the text. Shim coils and plexiglass oven are not shown. 
liwatt Sharp LT016 laser diode laser operating at $794.7 \mathrm{~nm}$. The laser is contained in a vacuum enclosure that is either evacuated or filled with nitrogen to avoid water condensation. The laser wavelength is tuned to the rubidium $D_{1}$ absorption line by lowering the temperature to approximately $-38^{\circ} \mathrm{C}$ using thermoelectric coolers. The laser temperature could be controlled to $0.01^{\circ}$ using a thermistor and feedback current controller circuit. The sample is optically pumped for typically two minutes. Rubidium spin polarization is transferred to xenon via spin-exchange interactions during the lifetime of van der Waals collisions. The mirror is then moved using a stepper motor and the cell is irradiated with unpolarized light from a $\mathrm{Rb}$ discharge lamp based on a design by Brewer [192]. The lamp probes the induced birefringence of the polarized rubidium vapor along the magnetic field. The rubidium polarization follows the xenon longitudinal magnetization (see Chapter 1). The polarized portion of the detection light is modulated using a photo-elastic modulator (model PEM080) [34] and then analyzed with a linear polarizer and detected by a silicon photo-detector. The signal is then fed into a lockin amplifier, (Stanford Research 575), which determines the bandwidth of the experiment and then digitized using a Textronix DSA602 digital oscilloscope.

Radio frequency pulses are applied to the sample using a transmitter consisting of a home-built pulse programmer card in a IBM compatible computer and a digital phase shifter. The pulse programmer consists of $1 \mathrm{k} \times 24$ bits of FIFO memory that contains timing and digital word information which is clocked out using an on-board $1 \mathrm{MHz}$ clock. The pulse programmer contrnls all timing of events in the experiment including opening and closing rf gates and triggering the data acquisition. The phase shifter produces 0,90, 180 and 270 degree phase-shifted rf pulses using digital flipflops, Frequencies of up to $1 \mathrm{MHz}$ may be derived from an external digital clock, and can be gated by the pulse programmer to create rf pulses. The pulses are filtered 
and sent to a tuned if circuit. Both rotating frame (nutation) and laboratory frame experiments can be performed.

We also used the "point-by-point" detection method [193, 194] whereby the evolution of the Zeeman or quadrupolar interaction in the transverse direction occurs during the time between two $90^{\circ},(\pi / 2)$ rf pulses which start and stop evolution. The evolution time in incremented between successive pump and acquisition cycles of the experiment. As illustrated in Figure 6.5, the evolution is initiated by a $\pi / 2$ $x$ pulse which converts the $z$ magnetization (enhanced by optical pumping in our case) to $x$ magnetization in the transverse plane. Evolution is later terminated by a $\pi / 2 \bar{x}$ pulse reconverting the $x$ component of the magnetization in the rotating frame back into $z$ magnetization that is then observed using optical detection, as discussed previously. The advantage of the point-by-point method is that the bandwidths of the evolution and observation times can be different. One can use a very short evolution time but still have good signal-to-noise ratios by using a narrow bandwidth in the detection phase of the experiment. The experimenter has total control over the evolution time which can be made very short to look at large bandwidths, or very long, to look at narrow resonances with high sensitivity.

Finally, to perform the $T_{1}$ measurements the lockin was set to a long time constant of about 1 second and the decay of $\left\langle I_{z}\right\rangle$ was observed after pumping. When necessary, the background signal from ${ }^{129} \mathrm{Xe}$ was reduced by applying a gradient rf field at the ${ }^{129} \mathrm{Xe}$ resonance frequency $(57 \mathrm{kHz}$ ) during the pumping period. Also, the signal was averaged using a 2-step phase cycle in which the odd acquisitions were shifted in phase by 180 degrees while the ${ }^{131} \mathrm{Xe}$ magnetization was inverted with an rf pulse prior to acquisition. This way, the ${ }^{131} \mathrm{Xe}$ signals added while the background signals and long-term drift tended to cancel on successive runs. 
$\mathbf{a}$

\section{nutation}

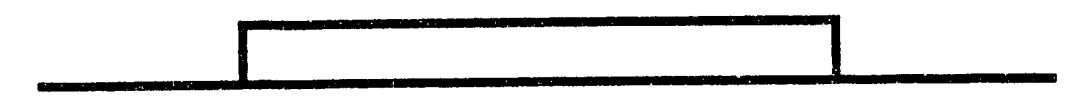

b phase reversed nutation

\begin{tabular}{c|c|c|c|c|c|c|}
\hline $2 \pi$ & $2 \pi$ & $2 \pi$ & $2 \pi$ & $2 \pi$ & $2 \pi$ \\
\hline & $\bar{x}$ & $\times$ & $\bar{x}$ & $\times$ & $\bar{x}$
\end{tabular}

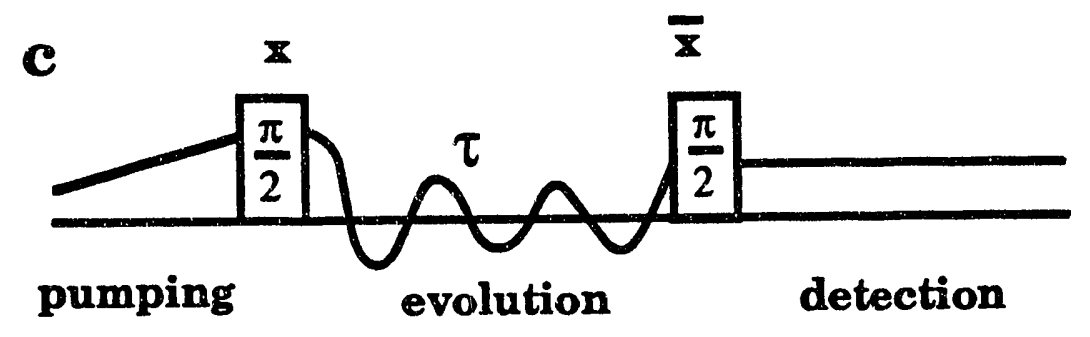

Figure 6.5: Pulses used in the various experiments. The nutation experiments consist of either (a) an single long pulse, or (b) an alternating sequence of $x$ and $\bar{x}$ pulses. The point-by-point experiments (c) consist of two $\pi / 2$ pulses separated by the desired evolution time $\tau$. A sample magnetization is draw in (a) as a solid line in its three periods of pumping, evolution and detection. 


\subsection{Results and Discussion}

\subsection{1 ${ }^{129} \mathrm{Xe}$ Nutation Er:periments}

Figure 6.6 shows the improvement one can achieve using the phase-reversed nutation as opposed to the the normal nutation experiments using nuclear spin $1 / 2$ ${ }^{129} \mathrm{Xe}$ shown with a nutation rate of $4 \mathrm{~Hz}$. The decay time is dominated by residual magnetic field inhomogeneities which, because of diffusion of the gas, reduce the dephasing time to approximately 4 seconds. In Figure 6.6 we have altered the nutation signal by alternating the phase of the applied rf pulse by 180 degrees, with each pulse length equal to the nutation period. The decay time increases to approximately 40 seconds and is now dominated by pulse imperfections such as residual rf field inhomogeneities.

\subsection{2 ${ }^{131} \mathrm{Xe}$ Nutation Experiments}

Figure 6.7 shows the nutation data at $120{ }^{\circ} \mathrm{C}$ for ${ }^{131} \mathrm{Xe}$ in a flat cell $3 \mathrm{~mm}$ high with added $\mathrm{H}_{2}$. The Fourier transform shows three peaks corresponding to the 3 frequencies of the quadrupole-perturbed Zeeman Hamiltonian [equation (6.7)]. The measured quadrupolar splitting for the $\mathrm{H}_{2}$ cell is about $50 \%$ larger than our results for a bare Pyrex cell, with no added hydrogen, that are in agreement with the results of Happer and coworkers. Further experiments are needed to determine the splitting dependence on the cell asymmetry as was done previously for bare Pyrex cells [84].

Quadrupolar splittings of the bare Pyrex cell and cells with added hydrogen were made at different temperatures, and the data fit to an Arrhenius behavior:

$$
\omega_{Q}=\omega_{Q_{0}} e^{-E_{a} / k T}
$$

with an activation energies $E_{a}$ of $-0.03 \mathrm{eV}$ for the bare cell, and $-0.12 \mathrm{eV}$ for the 

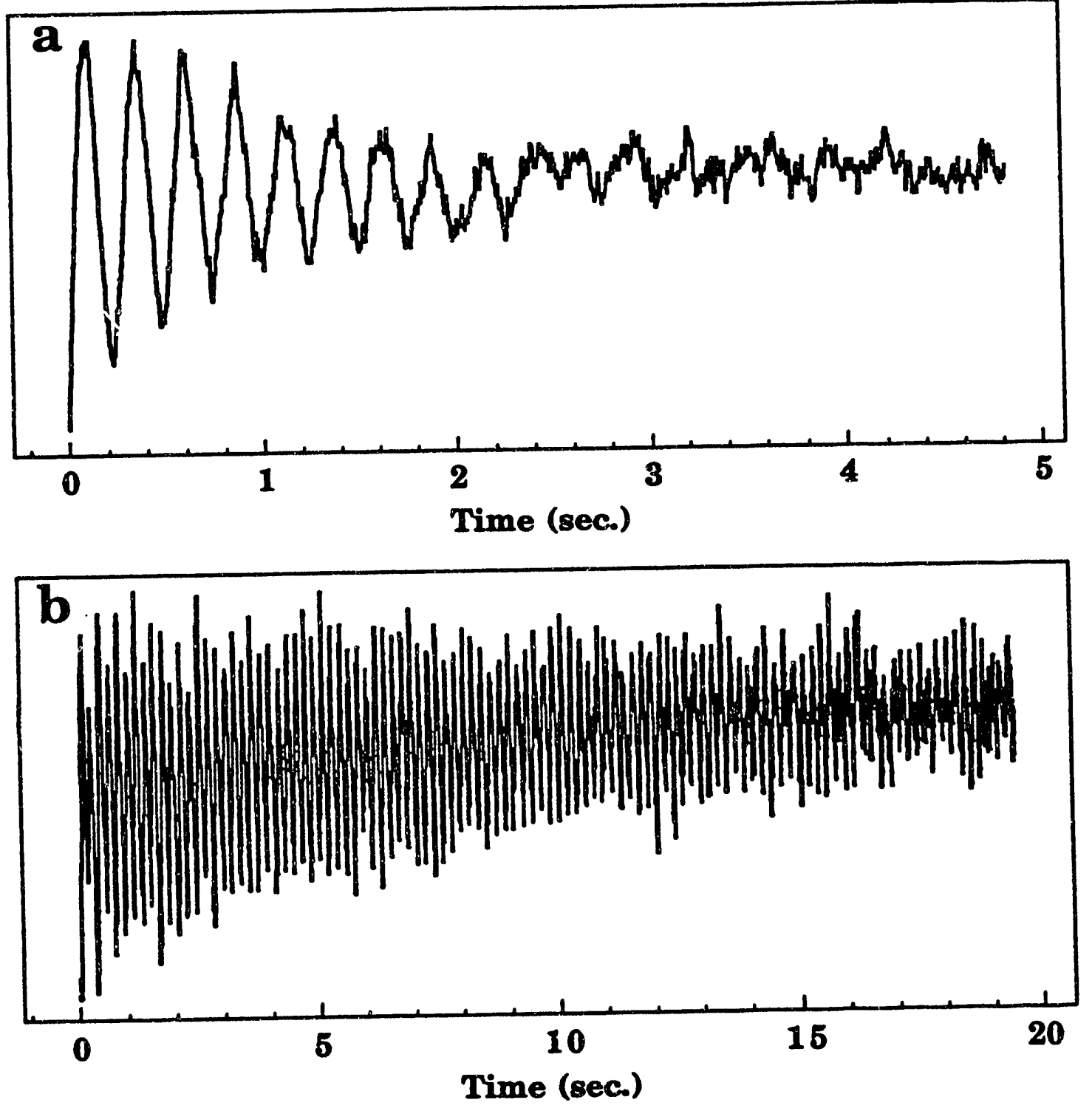

Figure 6.6: Optically-pumped and optically-detected ${ }^{129} \mathrm{Xe}$ nutation experiment. (a) The signal rapidly decays due to magnetic field inhomogeneities. (b) Reversing the rf nutation phase every nutation period refocuses the dephasing and increases the decay time. Notice the different time scales. 

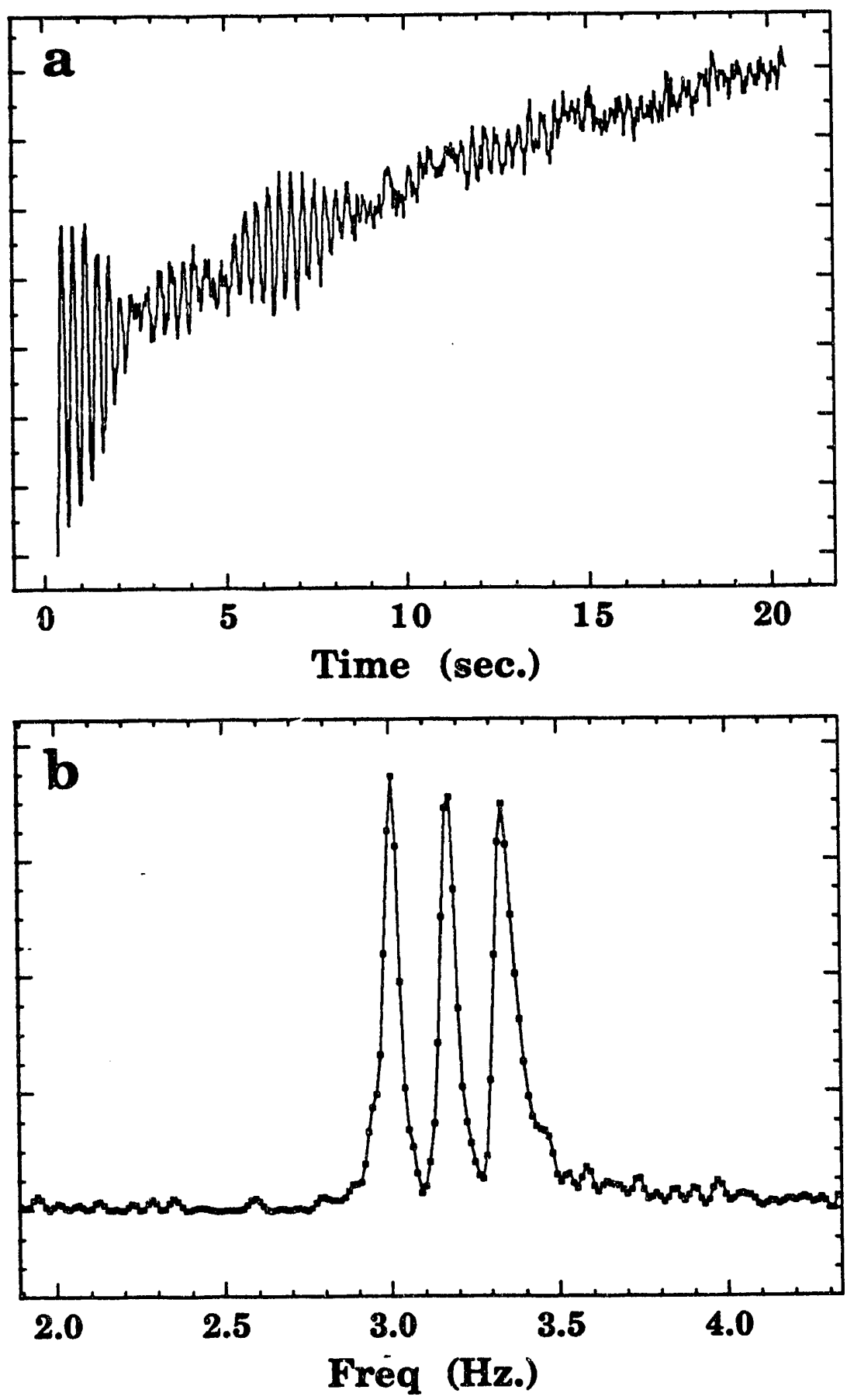

Figure 6.7: (a) ${ }^{131} \mathrm{Xe}$ nutation signal for $\mathrm{H}_{2}$ cell $0.3 \mathrm{~cm}$ height and $50 \mathrm{~mm}$ diameter at $120^{\circ} \mathrm{C}$. The ${ }^{129} \mathrm{Xe}$ background decay can be seen. (b) Fourier transformation of (a) shows quadrupolar splitting of $292 \mathrm{mHz}$. 
hydrogen cell, in agreement with the results of $\mathrm{Z}$. Wu et al. [181] and others [83]. The temperature dependent quadrupole splitting data for the $2 \mathrm{~mm}$ bare Pyrex cell and the 3mm Pyrex cell with added $H_{2}$ are shown in Figure 6.8

\subsubsection{Multiple Pulse Experiments}

Several methods of further increasing the resolution or bandwidth of the experiment were examined. First, higher order corrections to the simple phase-reversed nutation pulse sequence (Figure 6.5) can be made by using standard NMR composite pulse sequences. For example, the MLEV-16 sequence [195] is an example of an iterated sequence of rotations, $R$, and phase-reversed rotations, $\bar{R}$ :

\section{$R R \bar{R} \bar{R} \ddot{R} R R \bar{R} \bar{R} \bar{R} R R R \bar{R} \bar{R} R$}

Data from nutation experiments using $2 \pi$ and $8 \pi$ rotations plus a MLEV-16 a sequence is shown in Figure 6.9 for ${ }^{129} \mathrm{Xe}$ nutations. All of the pulses are weak in the sense that we follow the evolution of the xenon magnetization (using optical detection) during the pulses. The Rabi (nutation) frequency is chosen to be about $8 \mathrm{~Hz}$, so that it is larger than the rate of dephasing due to diffusion, and yet slow enough to be detected with good sensitivity by the lockin amplifier. The more complicated sequences are designed to be insensitive to pulse imperfections. However, from the Figure, it appears that the first sequence, simple phase alternation, works about the best, indicating that pulse imperfections still exist in the more complicated sequences.

\subsubsection{Increasing the Bandwidth}

The "point-by-point" experiment is an alternative method designed to increase the bandwidth. Point-by-point experiments are important in two dimensional [196] 

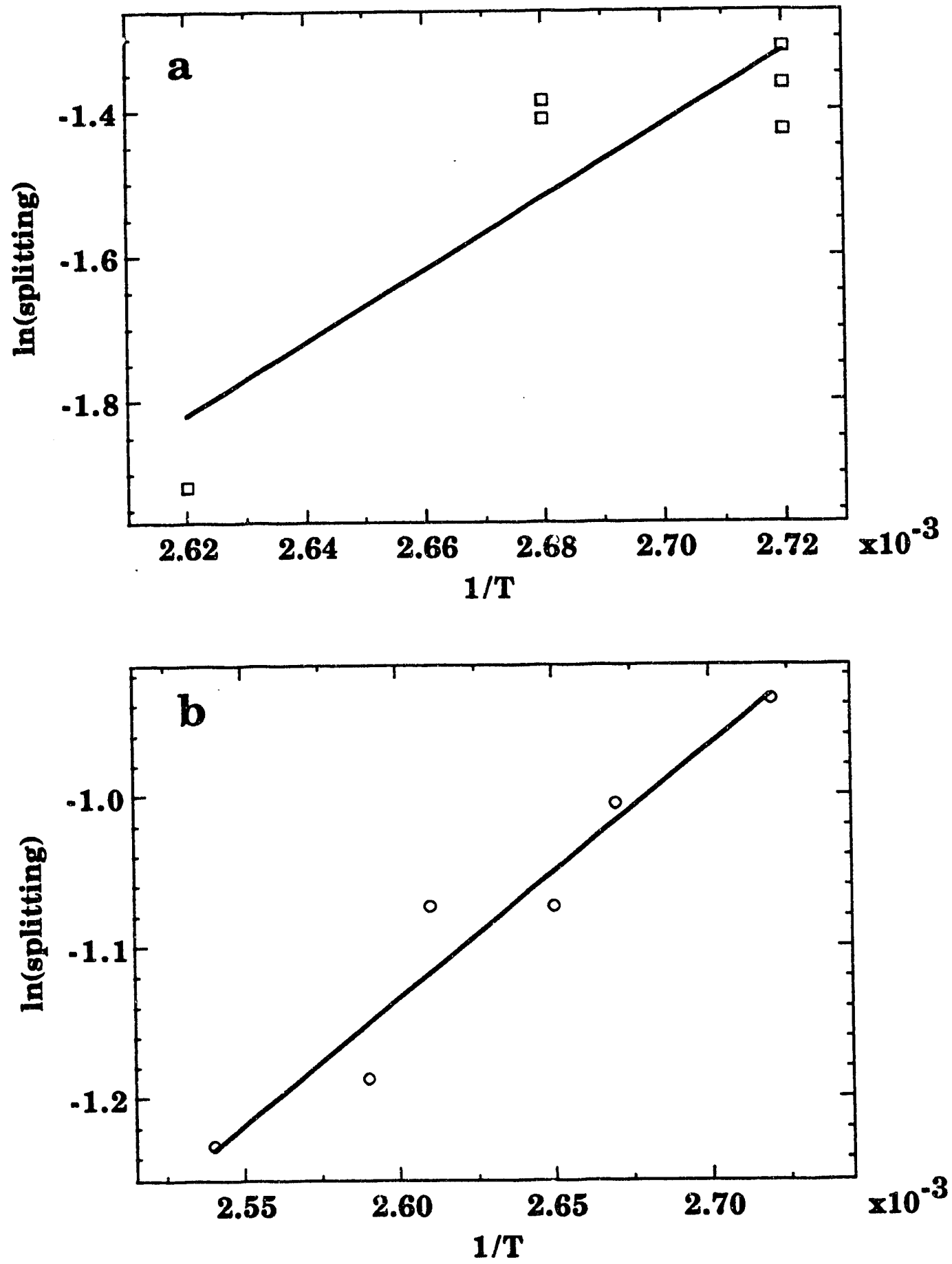

Figure 6.8: $\omega_{Q}$ versus cell temperature for (a) bare Pyrex cell and (b) Pyrex cell with added $\mathrm{H}_{2}$. 

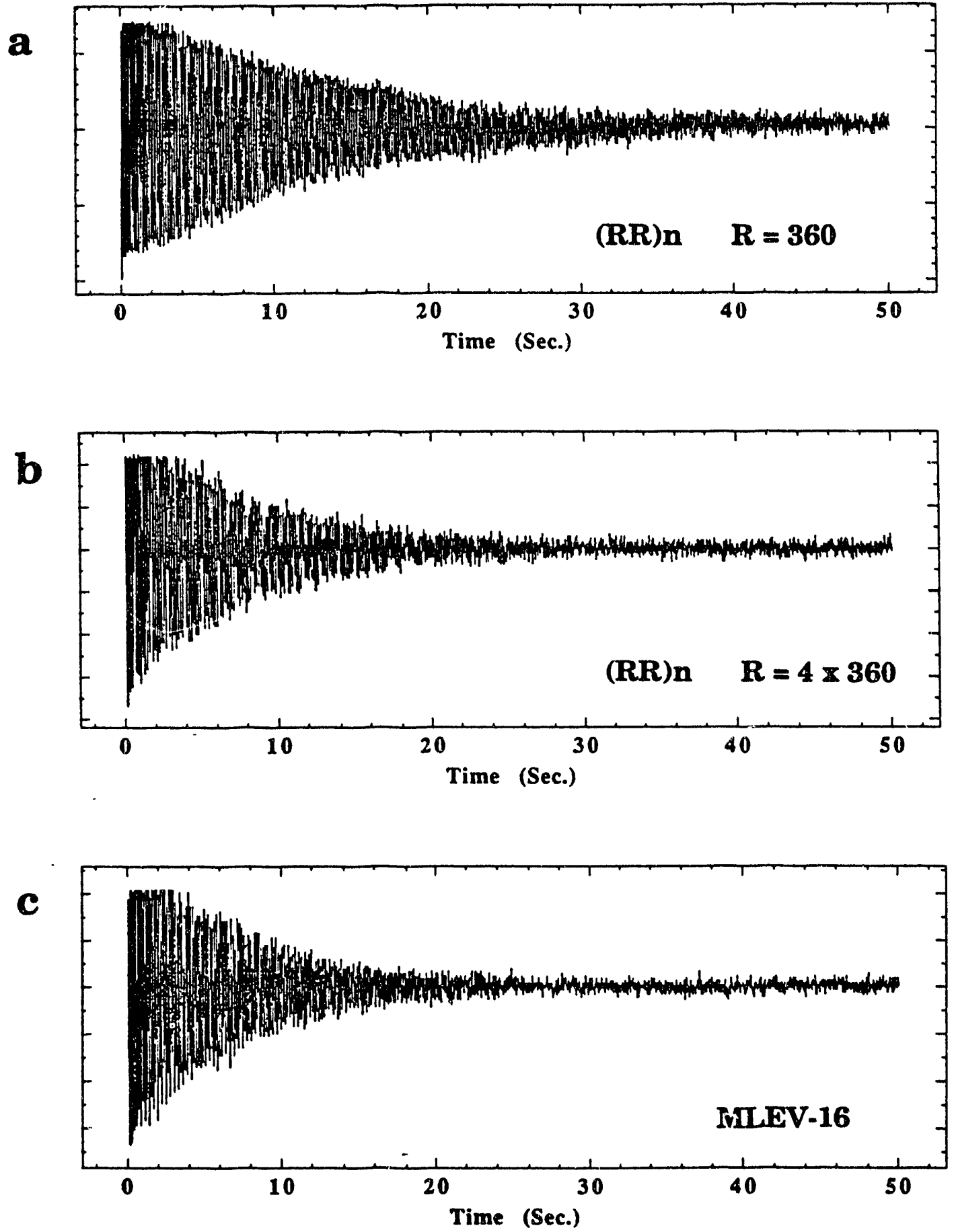

Figure 6.9: Several higher order pulse reversal experiments designed to average pulse imperfections. 
and zero-field NMR [194]. In our experiments on ${ }^{129} \mathrm{Xe}$, we investigated the effects of gas diffusion to determine the rf homogeneity. Figure 6.10 shows data from several such experiments. The first is the point-by-point FID that shows a decay time of about 3 seconds that is caused by rapid diffusion of the gas in the inhomogeneous magnetic field inside the pumping cell. The second, a CPMG [197] sequence, which is designed to refocus dephasing such as those caused by diffusion, shows a much longer decay time of approximately 40 seconds. Here the limitation is due to pulse imperfections and incorrect pulse lengths that still aiıow the xenon to dephase well before the rubidium induced $T_{1}$ limit of about 5 minutes at $100^{\circ} \mathrm{C}$.

In general, the point-by-point method allows a large flexibility in the possible experiments, however, it was not optimal for ${ }^{131} \mathrm{Xe}$ experiments because of the limited signal to noise ratio resulting from ${ }^{131} \mathrm{Xe}$ 's much inferior spin-exchange rate as compare to ${ }^{129} \mathrm{Xe}$ [83]. Another problem that appeared in the high resolution experiments are inherent drifts of the background polarized light signal that stemmed from temperature instabilities in the rubidium lamp and pumping cells. A reduction in time for the pump-probe experiments would be possible with higher power lasers than the $40 \mathrm{~mW}$ GaAlAs diode laser used in these experiments. Of course, normalizing the signal after pumping, and offsetting the drift between runs reduced this problem. Still, with our somewhat limited laser power, we found a second method to be more feasible.

\subsubsection{Phase-Reversed Nutation Experiments}

This second method, consisted of modifying the nutation experiments of Happer and coworkers [84]. Application of a nutation field in the $x$ direction and simultaneous observation of the $z$ magnetization by optical detection allows a sensitive method for ${ }^{131} \mathrm{Xe}$ quadrupolar experiments. As stated in the introduction, a major improvement 

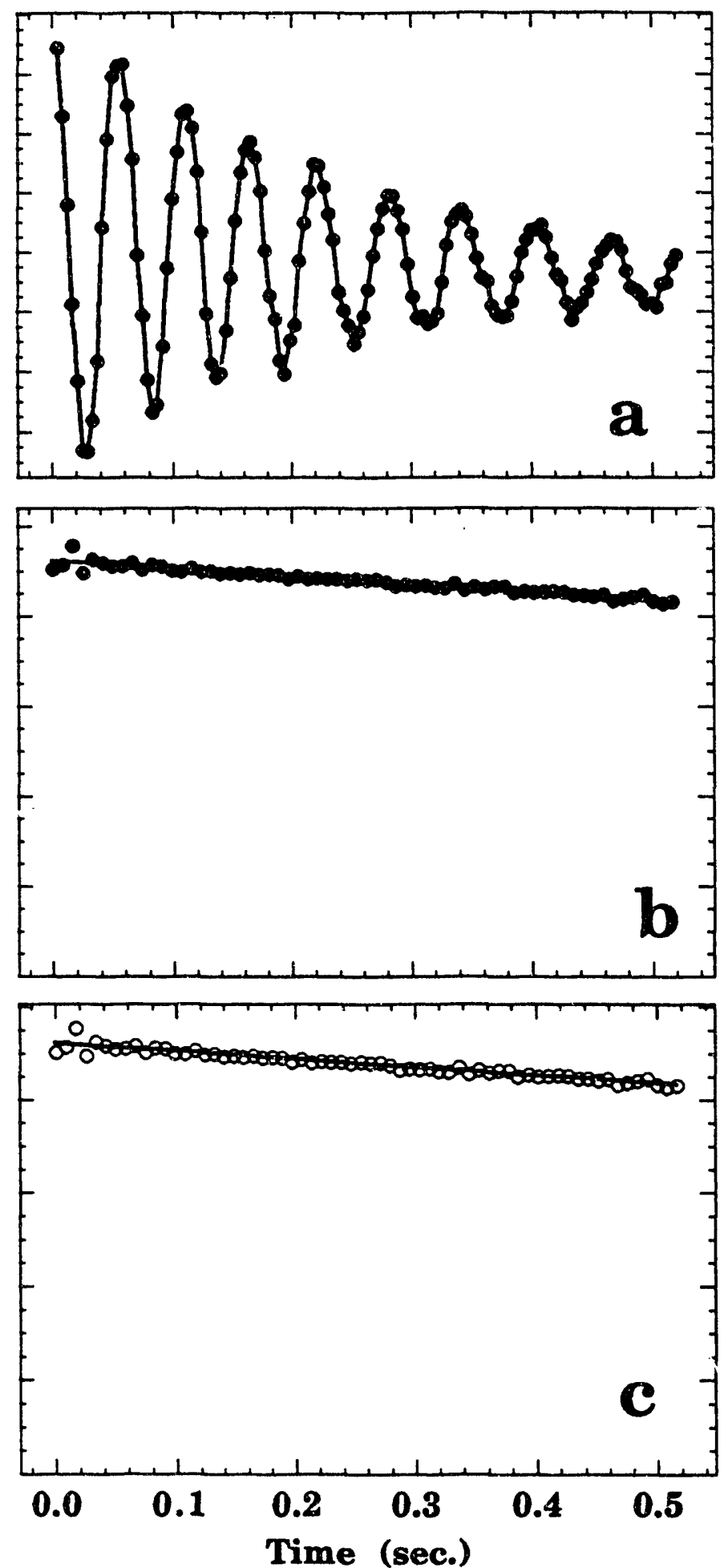

Figure 6.10: Point by point acquisition of optically pumped and detected ${ }^{129} \mathrm{Xe}$ signal. (a) Free induction decay, line width $=3 \mathrm{~Hz}$. (b) CPMG sequence (see text), decay time $=3 \mathrm{sec}$. (c) Spin-locking experiment, decay time $=8 \mathrm{sec}$. 
in this method was accomplished by reversing the phase of the rf field every nutation cycle, or $2 \pi$ rotation of the xenon magnetization. We experimented with shorter and longer periods but found 360 degrees to be optimal. Also, the spectra are more complicated for cycles that are not multiples of 180 degrees due to high frequency harmonics which are introduced by suddenly changing the sign of $d\left\langle I_{z}\right\rangle / d t$ which is zero only at 0 and 180 degrees. The lockin had to be set such that

$$
\omega_{1} \ll \frac{1}{\tau_{\text {lockin }}}
$$

Signal averaging was used and offsets due to drifts were reduced by reading in the dc value of the polarization from the lockin before acquisition. Accumulations of up to 20 nutations were coadded to improve signal-to-noise ratios. The data in Figures 6.7 and 6.8 were acquired using phase-reversed nutation.

\subsection{6 $T_{1}$ Measurements}

Several researchers have reported $[83,181,198,200]$ that $\mathrm{H}_{2}$ has the effect of lengthening the $T_{1}$ of ${ }^{131} \mathrm{Xe}$ in Pyrex glass cells. This probably results from reactions of $\mathrm{H}_{2}$ with paramagnetic centers on the surface of the glass, including rubidium, to form $\mathrm{M}-\mathrm{H}$ that reduces the paramagnetic relaxation of xenon at the surface. Nicol saw an increase in the relaxation time for ${ }^{129} \mathrm{Xe}$ in Pyrex cells by adding $\mathrm{H}_{2}$, although the relaxation time decreased in Corning 1720 cells [199]. For ${ }^{129} \mathrm{Xe}$ optical pumping experiments, the largest effect is obtained by coating the cells witk Surfrasil [85] which is thought to cause the sarne shielding mechanism for it also has the effect of substantially lengthening the relaxation time. The ${ }^{131} \mathrm{Xe} \mathrm{T}_{1}$ data for the $\mathrm{H}_{2}$ cells is shown in Figure 6.11, with a fit using the function from equation 6.1 . The derived value of $\sigma_{e x} \bar{\nu}$ is in agreement with results of Volk et al. [86], however more data is needed to definitively compare our results for the other parameters $\Gamma$ and $\left(T_{2}^{\prime}\right)^{-1}$. 


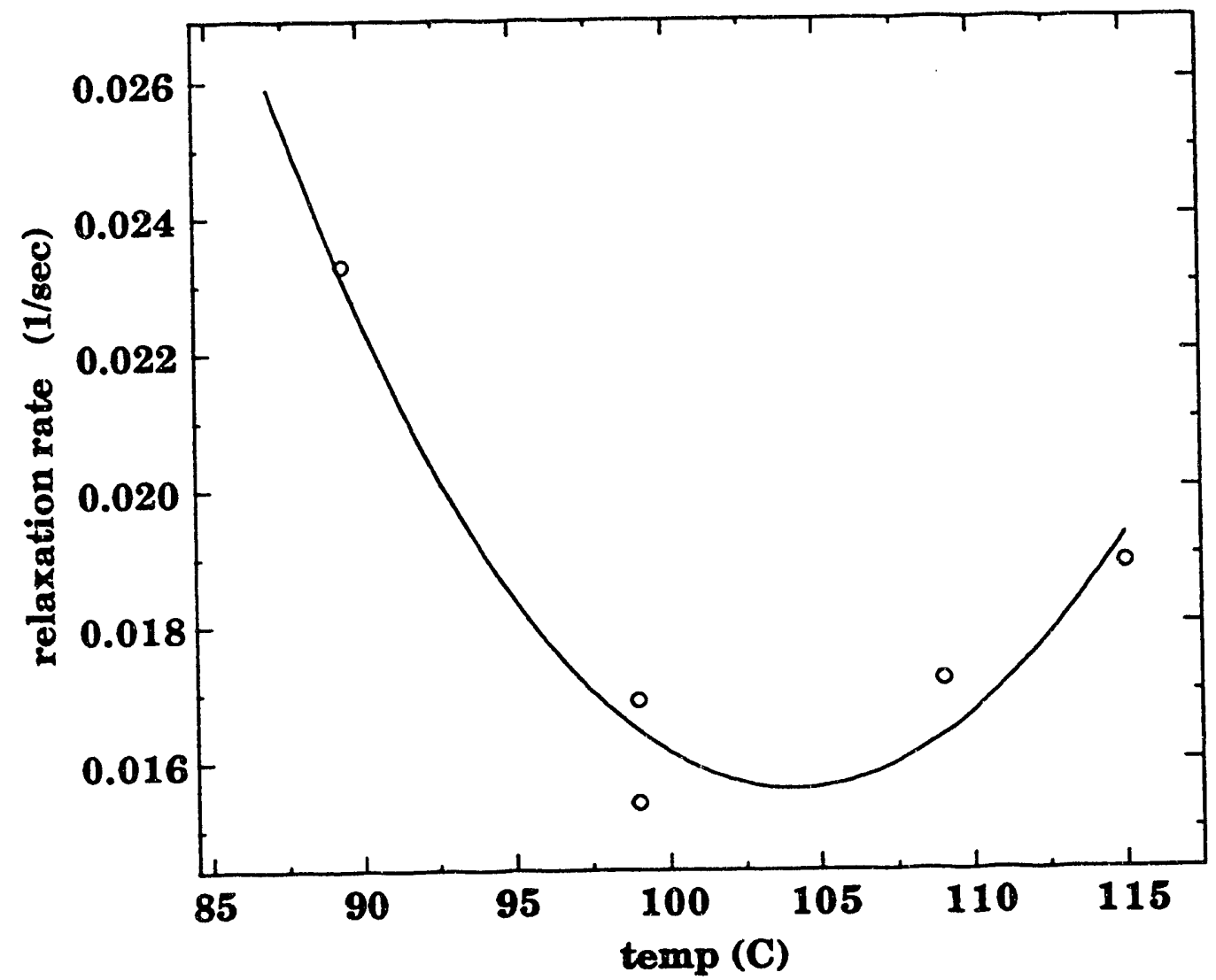

Figure 6.11: $\mathrm{T}_{1}$ data for the $\mathrm{H}_{2}$ cell as a function of temperature. 


\subsection{Conclusions}

A variety of different experimental methods have shown that the optical-NMR spectrometer described in this chapter can yield valuable information concerning ${ }^{131} \mathrm{Xe}$ quadrupolar interactions at the glass surface. Major improvements in these experiments were accomplished by multiple-pulse coherent averaging methods, such as phase-reversed nutation.

An interesting result is that cells which have added hydrogen gas have quadrupolar splittings that are larger than those for bare Pyrex cells. This is seemingly at odds with the idea that the cause of the increased $T_{1}$ relaxation times measured in the same cells is probably due to the reaction of hydrogen with paramagnetic impurities on the glass surface. Finally, the point-by-point detection method can be used to increase the bandwidth of the experiment, assuming the signal to noise ratio is otherwise sufficient. 


\section{Chapter 7}

\section{Optically-Pumped Xenon Detected in High Field}

\subsection{Introducioion}

In this chapter. experiments are discussed that combine the methods of optical pumping with conventional NMR detection to realize a novel and interesting technique for surface studies [5]. Recent experiments in two areas have created the possibility of extending ihe already powerful methods of pulsed NMR [13, 201] to the study of surfaces of materials with moderate to low surface areas. As discussed in Chapters 1 and 6, Happer and coworkers [21], ourselves, [5] and others [20], have polarized xenon and other noble gas atoms via collisions with optically pumped rubidium [79] in order to study surface interactions of ${ }^{131} \mathrm{Xe}$ with glass cell walls $[83,84,89,181,202]$ and to measure fundamental physics quantities $[182,203,204]$. More recently, Cates et al. have studied the $T_{1}$ of solid ${ }^{129} \mathrm{Xe}$ by transfer of optically pumped xenon gas to a magnet [78]. Meanwhile, as described in Chapter 3, there has been much activity in the use of conventional ${ }^{129} \mathrm{Xe}$ NMR to help elucidate the 
structure of zeolites [205] and clathrates [101], as well as to characterize dispersed metals [206] and adsorbed guest molecules inside Na-Y zeolite [123]. Because of its large diameter and the sensitivity of its NMR parameters to perturbations, xenon is a useful probe-of microscopic chemical environments. The main drawback of these NMR experiments is that, unless the temperature is extremely low [207], and $T_{1}$ is short, the sensitivity is unfavorable, and one is often limited to systems with high surface area $[208,72]$.

In this chapter, the results of experiments on pulsed high field NMR of laserpumped ${ }^{129} \mathrm{Xe}$ are presented in which we have observed spectra corresponding to low pressure xenon gas and to the adsorption of xenon on powdered solids of moderate surface area (less than $20 \mathrm{~m}^{2} / \mathrm{g}$ ), a result of potential significance in surface science and catalysis. Ordinary amorphous solid materiais with particle sizes on the order of 10 microns have surface areas around $1 \mathrm{~m}^{2} / \mathrm{g}$, so that adsorption studies on a wide range of materials should be possible using this technique.

\subsection{Signal Enhancement: A Demonstration}

A direct comparison of the ${ }^{129} \mathrm{Xe}$ NMR signal in xenon gas was made with and without optical pumping in our 4.4 Tesla magnet in order to estimate the enhanced xenon nuclear polarization afforded by optical pumping. In Figure 7.1(a) the conventional Fourier transform NMR spectrum of a $3.3 \mathrm{~cm}^{3}$ sample volume containing 206 torr of natural abundance $(26 \%){ }^{129} \mathrm{Xe}$ at room temperature. Approximately 1000 torr of oxygen was added to reduce the ${ }^{129} \mathrm{Xe}$ relaxation time to 1.3 seconds [209], which also had the effect of shifting the xenon resonance frequency by $80 \mathrm{~Hz}$ relative to the pure gas, in agreement with reference [209]. After 100 acquisitions, the signal to noise ratio is 14 indicating that the detection limit for a signal to noise 


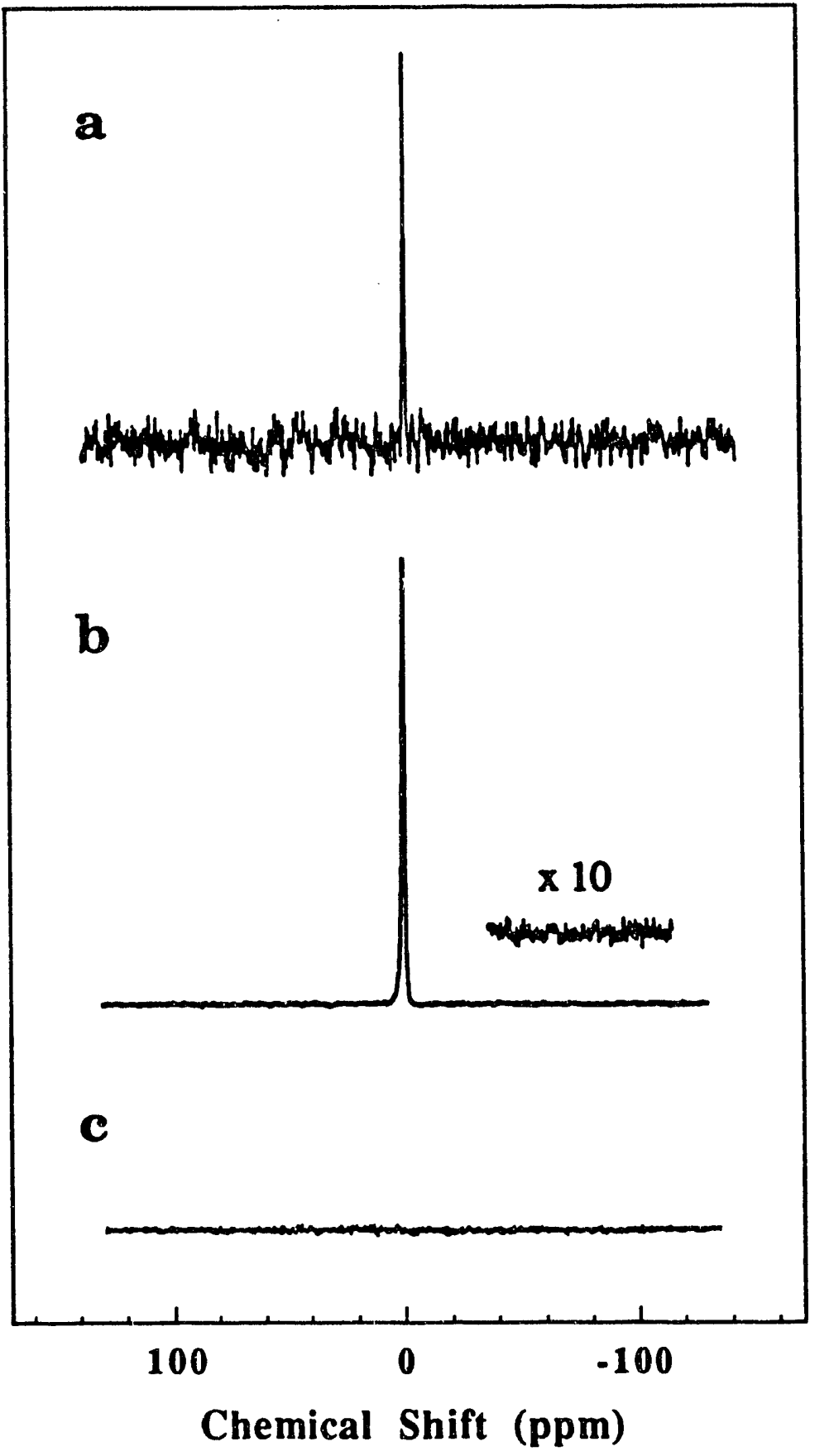

Figure 7.1: Room temperature ( $298 \mathrm{~K})$ NMR spectra of naturai abundance ${ }^{129} \mathrm{Xe}$ : (a) using conventional NMR 100 acquisitions of a $3.3 \mathrm{~cm}^{3}$ sample containing 206 torr xenon and 1000 torr oxygen; (b) single acquisition of optically-pumped xenon, $8 \mathrm{~cm}^{3}$ containing 32 torr; (c) same sample as (b) but after nuclear polarization has decayed to equilibrium. 
ratio of 1 is about $4 \times 10^{18}{ }^{129} \mathrm{Xe}$ nuclear spins for our spectrometer (corresponding to about $3 \times 10^{13} 100 \%$ polarized ${ }^{129} \mathrm{Xe}$ nuclear spins at $4.4 \mathrm{Tesla}$ ) with a spectral bandwidth of $40 \mathrm{kHz}$. The line width is $29 \mathrm{~Hz}$ (FWHM), due primarily to residual field inhomogeneities over the sample volume, and is shown in Figure 7.1(a) with a $20 \mathrm{~Hz}$ Lorentzian line broadening. The spectrum for a single acquisition of optically pumped, natural abundance ${ }^{129} \mathrm{Xe}$ at only 32 torr, with no added oxygen gas, is shown in Figure 7.1(b), and has a signal to noise ratio of 300 with a line width of $39 \mathrm{~Hz}$. In Figure 7.1(c), a single acquisition of the same optically-pumped xenon sample is shown after the optical polarization has decayed to equilibrium. We have estimated the spin-lattice relaxation time $\left(T_{1}\right)$ for pure, gas phase ${ }^{129} \mathrm{Xe}$ at high field to be more than 20 minutes in our coated glass (see text below), $8 \mathrm{~cm}^{3}$ sample region. The enhancement over conventional xenon NMR in our apparatus is about 750 . Our observed NMR signals of the xenon gas correspond to a nuclear polarization is about $0.5 \%$, which, accounting for our laser power and operating conditions, is consistent with results of Happer and coworkers [79]. Higher polarizations, and thus larger enhancements, can easily be achieved by pumping a smaller pressure of xenon gas and shimming the residual magnetic field inhomogeneities. At the prtusures (200 to 600 torr) and magnetic fields (250 gauss) used in this demonstration described here, the spin-exchange transfer efficiency is reduced almost to the binary collision limit (see Chapter 1 for details).

\subsection{Experimental Details}

\subsubsection{The Apparatus}

The apparatus, shown schematically in Figure 7.2, consists of an optical pumping cell located in the fringe magnetic field (250 gauss) underneath a superconducting 
NMR magnet and a sample/detection cell located in high magnetic field. The two regions are connected by a glass tube and separated by a series of stopcocks. Separate connections allow the evacuation and pressure measurement of the sample region. Natural abundance rubidiura is contained in a reservoir region connected with the pumping cell, and is heated in an oven with warm flowing nitrogen gas. The procedure for preparing the optical pumping cell is similar to that described in Chapter 6. The rubidium is optically pumped to a polarization level approaching $100 \%$ using circularly polarized light from a temperature-stabilized, 40 milliwatt, single-mode semiconductor diode laser (Sharp model LT016) operating at $794.7 \mathrm{~nm}$. The laser illuminates approximately $2 / 3$ of the cell volume. The entire glass manifold has been coated with Surfrasil [85], a siliconizing agent that reduces the wall-induced relaxation rate of ${ }^{129} \mathrm{Xe}$ to less than 2 per hour. The cylindrical optical pumping cell of approximately $11 \mathrm{~cm}^{3}$ volume is filled with between 100 and 600 torr of natural abundance or enriched (70\%) spin-1/2 xenon-129. A Helmholtz pair of shim coils were used in the low pressure experiments to reduce the fringe magnetic field to approximately 75 gauss which had the effect of increasing the spin exchange efficiency [28].

\subsubsection{NMR Probe}

A schematic drawing of the probe used in these experiments is shown in Figure 7.3. The main features of the probe are the Macor ceramic coil support, glass Dewar, Dewared stainless steel transfer line, and glass transfer line for the polarized xenon. We found that at very cold temperatures, the xenon had a tendency to freeze out on to the walls of the glass tube on the way to the sample region. A Teflon box was constructed to limit the coldest part of the probe to the immediate sample region.

A rf-shielded thermocouple was inserted into the Teflon box to monitor and con- 


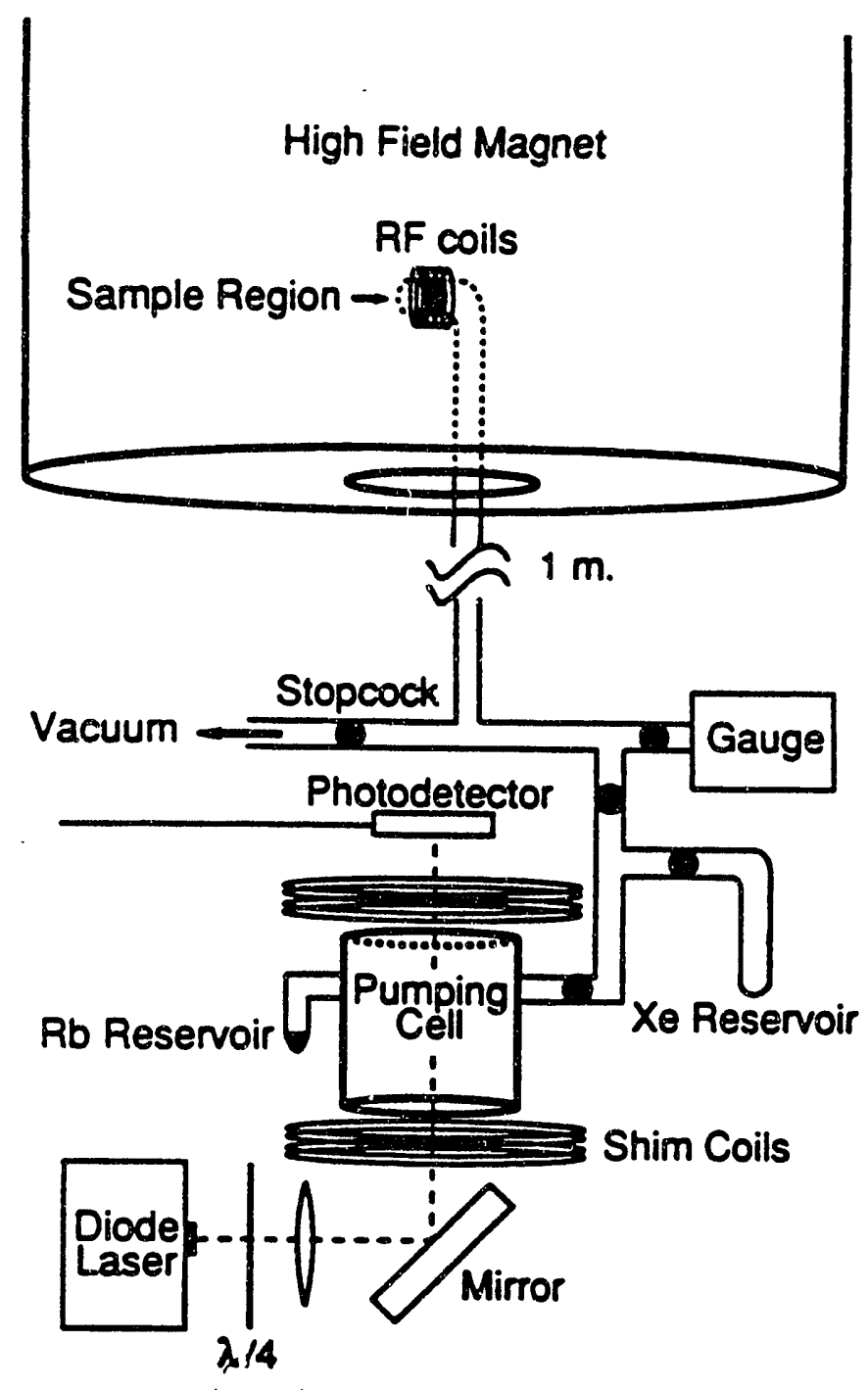

Figure 7.2: Schematic diagram of the experimental apparatus. Circularly polarized $794.7 \mathrm{~nm}$ laser light is focused onto the optical pumping cell. A silicon photo detector is used to detect the rubidium absorption. The oven used to heat the pumping cell, and the nitrogen cooling system for the sample region are not shown. Shim coils can be used to cancel gradients and/or reduce the overall magnetic field in the pumping cell. 


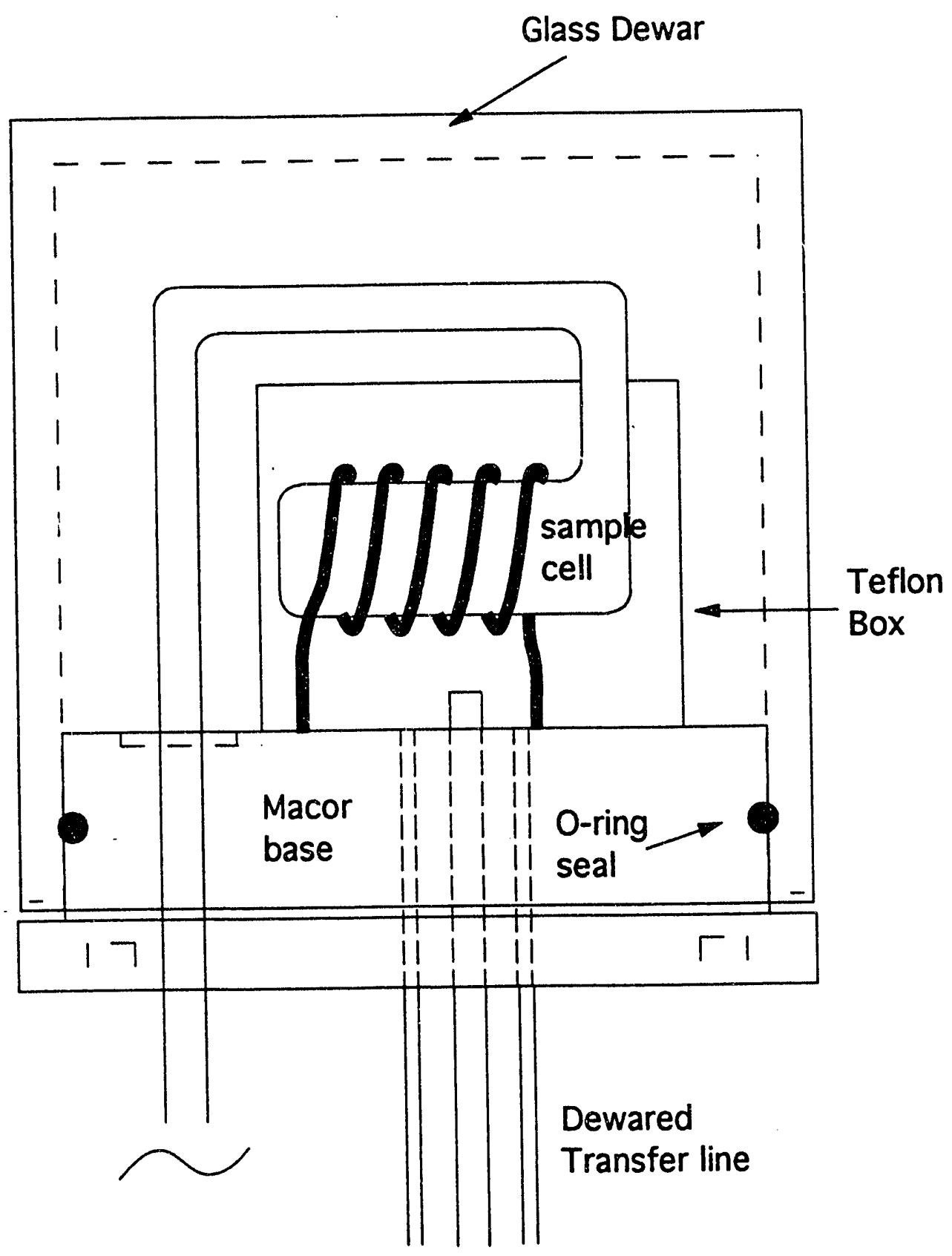

Figure 7.3: Drawing of the probe used for xenon optical pumping experiments in high field. 
trol the temperature at the sample. We found that careful shielding of the thermocouple was necessary to prevent radio frequency pickup noise from coupling to the rf coil.

A double resonance rf circuit was built based on a design by Doty and coworkers [210], had the advantage of good efficiency at the xenon resonance frequency while allowing for a large sample volume of about $8 \mathrm{~cm}^{3}$. Later, a smaller coil $\left(3 \mathrm{~cm}^{3}\right.$ volume) was also employed for better efficiency on the high frequency side for preliminary cross polarization experiments.

\subsubsection{Timing Considerations}

The timing diagram for the experiment is shown in Figure 7.4. We normally optically pump for approximately 30 minutes at temperatures between $80^{\circ} \mathrm{C}$ and $120^{\circ} \mathrm{C}$, so that the absorption of incident pumping light is between $50 \%$ and $100 \%$. After pumping, the cell is rapidly cooled to approximately $40^{\circ} \mathrm{C}$ to reduce the vapor pressure of rubidium in the pumping cell. The ${ }^{129} \mathrm{Xe}$ does not relax appreciably during this cooling period. The stopcock is then opened and polarized xenon flows (in less than 1 second) up to the sample region where it can adsorb onto the sample. For the surface samples, we typically wait for $30-60 \mathrm{sec}$. to allow the xenon to reach an equilibrium adsorption on the surface before acquiring data. Spectra are recorded by Fourier transformation of the signal following a radiofrequency pulse at $51.4 \mathrm{MHz}$. The NMR signal is typically obtained in a single scan with sufficient signal-to-noise ratio. 


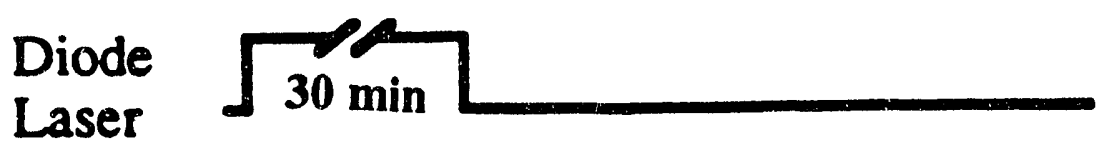

Gas
Stopcock

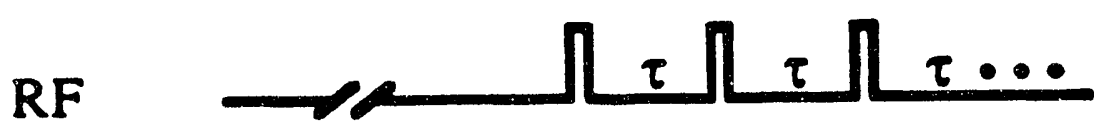

Figure 7.4: Timing diagram for the high field experiment. 


\subsubsection{Recycling the Experiment}

After data acquisition, the sample is allowed to warm up and the xenon is collected by freezing it into the xenon reservoir (see Figure 7.2) using liquid nitrogen. This gas shuttling method is obviously not the most efficient in terms of time. One could think of a variety of flow-type systems where the yenon is continuously being pumped, adsorbed, desorbed, decontaminated (see next paragraph) and so forth. However for the samples which need to be cooled, the massive heat content of the probe and sample region make this the rate limiting step in the experiment. Also, in terms of hardware, the up and back design is not too difficult. I imagine that other, more efficient arrangements will soon be implemented.

Sometimes, the xenon becomes slightly contaminated during its contact with the surface. This is most often due to small quantities of water which remain after evacuation, or which leak in to the vacuum system, and which may become dislodged from the surface due to collisions with xenon. Water reacts quickly with rubidium, so before returning the xenon to the pumping cell it is necessary to decontaminate the xenon. To this end, a small amount of rubidium metal is placed in the xenon reservoir tube and warmed by a heat gun for several minutes to vaporize some rubidium. The water and other contaminants react in the xenon reservoir region and the xenon becomes purified. Otherwise, a very small amount of contaminant will diminish the pumping efficiency to a very large extent. The rubidium concentration in the pumping cell is only $10^{12} \mathrm{~cm}^{-3}$, or about $10^{-5}$ torr, so the xenon must be very pure. We can monitor the contamination level in the pumping cell by simply observing the rubidium absorption of the laser light at a fixed temperature. 


\subsubsection{Sample Treatments}

A main concern of the experiments described in this chapter, and in surface studies in general, is the preparation of a clean surface. In most surface science experiments, the sample, typically a metal crystal, is annealed, sputtered, polished (or a combination of these treatments) prior to the adsorption of gases. In working with powdered materials, however, it is impossible to perform such treatments. In fact, an interesting question to address is whether carefully-prepared single metal crystal surfaces actually mimic situations found in nature, where air is usually present. Nevertheless, we did try to treat our samples in a manner consistent with other studies of powdered materials. This meant evacuation at room temperature, or high temperatures to eliminate as much as possible, physisorbed molecules such as water or oxygen. For samples such as benzanthracene, room temperature evacuation was sufficient to reduce the pressure to less than $10^{-5}$ torr. At this pressure, water was not present in sufficient quantities to contaminate the xenon such that the optical pumping was affected, which, incidentally, turned out to be a sensitive measure of the water or other contaminants presence (also, see below). More strongly adsorbing surfaces such as polyacrylic acid required heating. We found that $80^{\circ} \mathrm{C}$ was the high temperature limit for dehydration without degradation of the polymer. As will be discussed below, the surface treatment of polyacrylic acid was important in its effect upon the xenon environment as detected in the NMR spectra.

\subsubsection{Water Contamination}

An interesting use of the optical pumping technique is the characterization of surfaces after treatment. For example, the polyacrylic acid surface has a large affinity for water adsorption that is evident in the ${ }^{129} \mathrm{Xe}$ spectra. Figure $7.5(\mathrm{a})$ shows the 

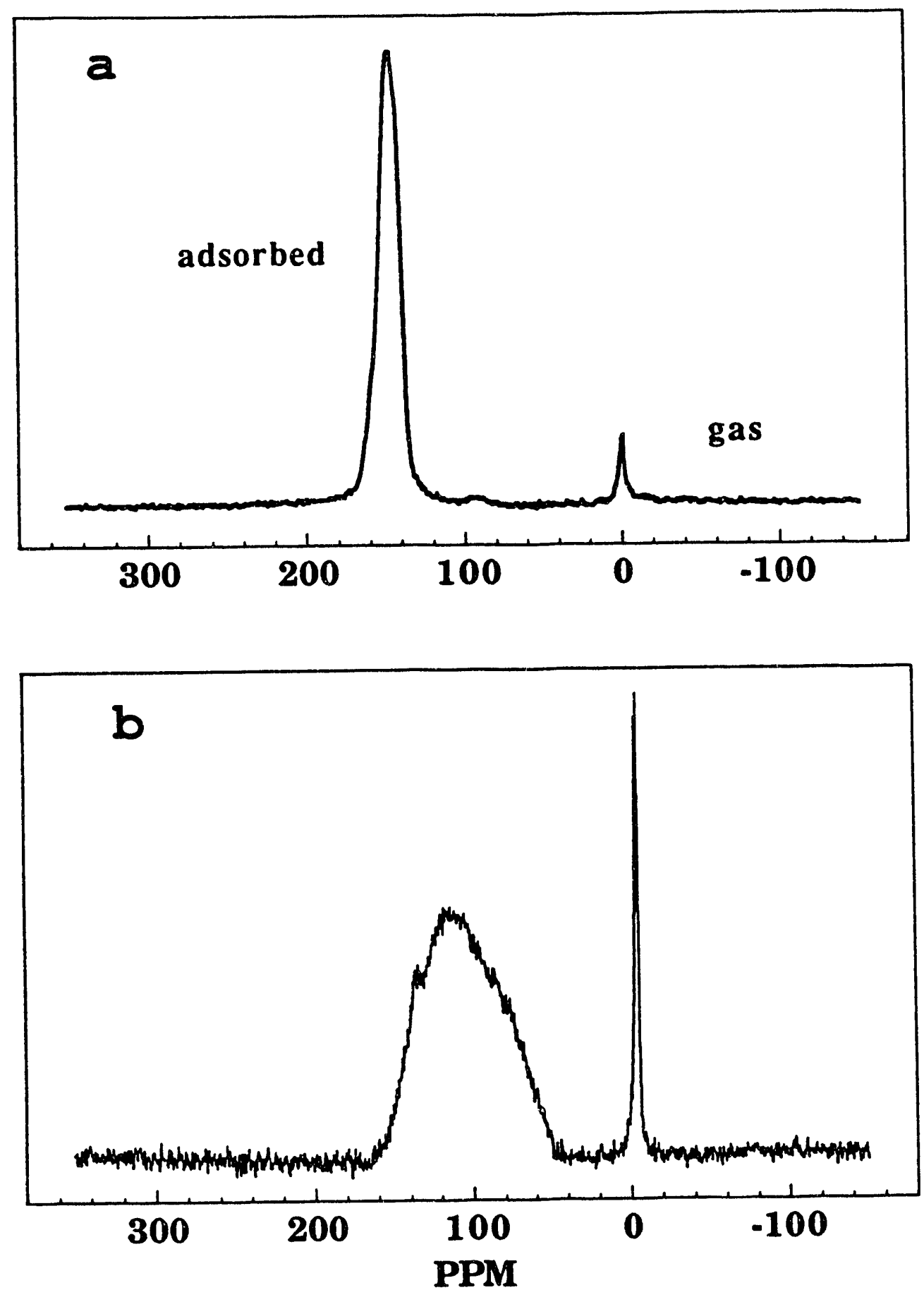

Figure 7.5: ${ }^{129} \mathrm{Xe}$ NMR spectra for xenon adsorbed onto polyacrylic acid that had been previously evacuated at (a) room temperature for several hours, and (b) overnight at $80^{\circ} \mathrm{C}$. 
spectrum for xenon adsorbed on polyacrylic acid that had been evacuated at room temperature for about 2 hours. The chemical shift range is very large, over $100 \mathrm{ppm}$, indicating that xenon experiences a variety of interactions at the surface that are not averaged on the NMR time scale of milliseconds. This is in sharp contrast to the spectrum shown in Figure 7.5(b), or the earlier spectra shown in Figure 7.8, where the polyacrylic acid had been evacuated overnight at $80^{\circ} \mathrm{C}$. The xenon spectra are much narrower due to the much more homogeneous surface with which xenon comes into contact. In Figure $7.5(\mathrm{~b})$, the chemical shift is about $136 \mathrm{ppm}$, and corresponds to the high range of chemical shifts in the spectrum above. Xenon probably experiences a reduced interaction due to the presence of water at the surface of the polyacrylic acid. It was also observed that the $T_{1}$ relaxation time for xenon was dramatically reduced by the surface water molecules making the experiment somewhat difficult to perform under equilibrium conditions.

\subsection{Results}

\subsubsection{Benzanthracene}

Figure 7.6 shows spectra for polarized, enriched ${ }^{129} \mathrm{Xe}$ in the presence of $0.96 \mathrm{~g}$ of 1,2-benzanthracene (surface area about $0.5 \mathrm{~m}^{2} / \mathrm{g}$ as estimated by average particle size from electron micrographs and by nitrogen BET isotherm) at several temperatures. At room temperature only a narrow peak due to gas phase ${ }^{129} \mathrm{Xe}$ is observed at the same resonance frequency as the optically-pumped pure xenon gas. We use this frequency as the reference, defined as 0 ppm (parts per million) chemical shift. When polarized xenon gas is admitted to the sample at lower temperatures, additional peaks appear. At $158 \mathrm{~K}$, xenon adsorbed onto the surface has a resonant frequency which is shifted by $10 \mathrm{ppm}$ compared to the gas peak. At $153 \mathrm{~K}$, the peak 


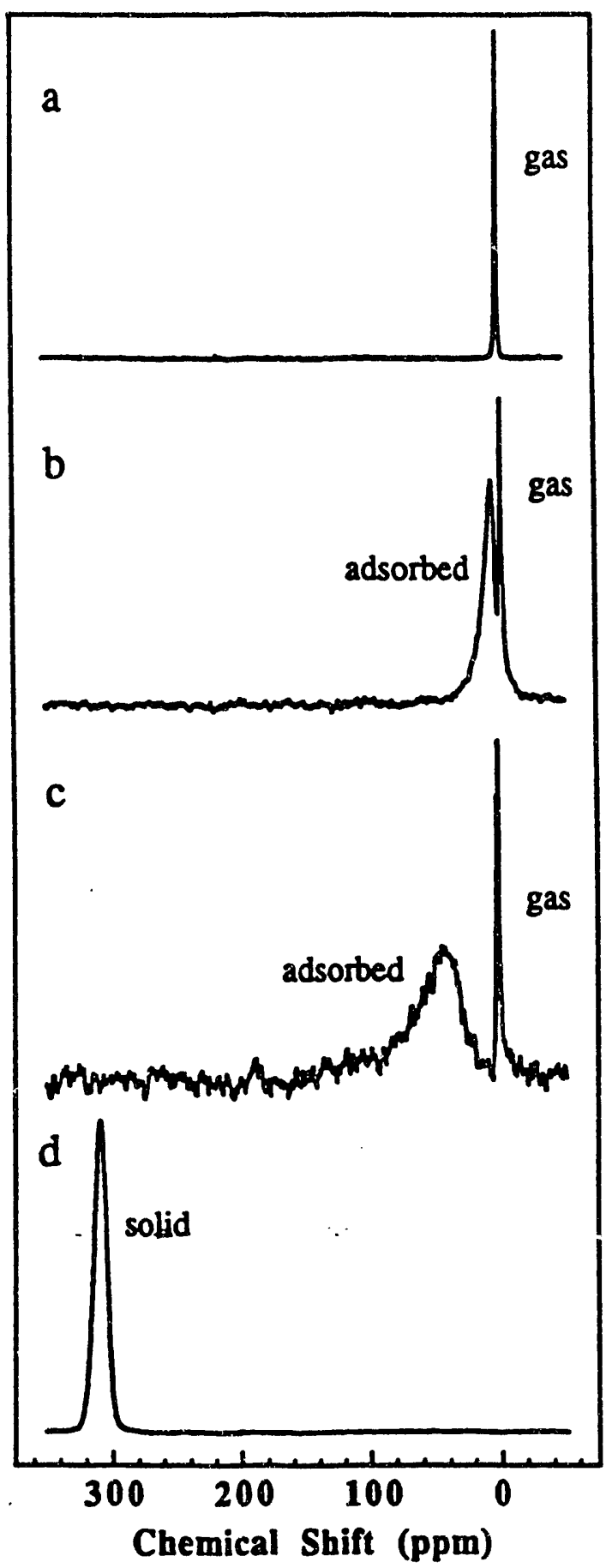

Figure 7.6: NMR spectra of enriched ${ }^{129} \mathrm{Xe}$ in the presence of powdered benzanthracene following optical pumping: (a) xenon gas peak only, $\mathrm{T}=298 \mathrm{~K}, \mathrm{P}=$ 20 torr; (b) xenon gas and adsorbed, $\mathrm{T}=158 \mathrm{~K}, \mathrm{P}=21$ torr; (c) xenon gas and adsorbed, $\mathrm{T}=153 \mathrm{~K}, \mathrm{P}=22 \mathrm{torr}$; (d) solid xenon, $\mathrm{T}=123 \mathrm{~K}$. 
associated with adsorbed xenon is observed at $32 \mathrm{ppm}$ relative to the gas and has a somewhat asymmetric line shape. The chemical shift and line width increase with decreasing temperature, indicating a continual increase in the residence time of the adsorbed xenon. The line shape of the adsorbed xenon signal at $153 \mathrm{~K}$ may arise from a distortion of the xenon electron cloud on the surface of the benzanthracene. Indeed, Ripmeester has recorded chemical shift anisotropies as large as $160 \mathrm{ppm}$ for xenon trapred in $\beta$-quinol clathrate [3]. Below $130 \mathrm{~K}$, we detect the NMR signal of polarized solid xenon with a temperature-dependent line width in agreement with previous work $[56,57]$, corrected for isotopic enrichment. However, the measured chemical shift of the solid is $10 \mathrm{ppm}$ larger than in references $[56,57]$, perhaps due to susceptibility effects.

\subsubsection{Na-Y Zeolite}

It is also possible to study surfaces on which xenon has relatively short spin-lattice relaxation times. Figurc 7.7 shows the spectrum of ${ }^{129} \mathrm{Xe}$ adsorbed in $\mathrm{Na}-\mathrm{Y}$ zeolite at 16 torr. The measured $T_{1}$ relaxation time for xenon in $\mathrm{Na}-\mathrm{Y}$ zeolite is about 100 milliseconds, so several data acquisitions with a short delay (100 ms) between them were made to observe the signal. Multiple data acquisitions may be used to follow the time development of the xenon adsorption. In fact, the line width of the adsorbed xenon signal in $\mathrm{Na}-\mathrm{Y}$ zeolite decreases from $450 \mathrm{~Hz}$ in the first scan after the xenon arrive.i in the probe, to $300 \mathrm{~Hz}$ taken $200 \mathrm{~ms}$ later. The chemical shift of $58 \mathrm{ppm}$ is measured at a pressure of 16 torr. This is the lowest pressure for xenon NMR reported to date, and the chemical shift is in agreement with the reported values (extrapolated to zero xenon pressure) of $58.5 \pm 0.5 \mathrm{ppm}$ for xenon adsorbed in $\mathrm{Na}-\mathrm{Y}$ zeolite [2].

Our observed optical pumping enhancement for xenon arisorbed in $\mathrm{Na}-\mathrm{Y}$ zeolite 


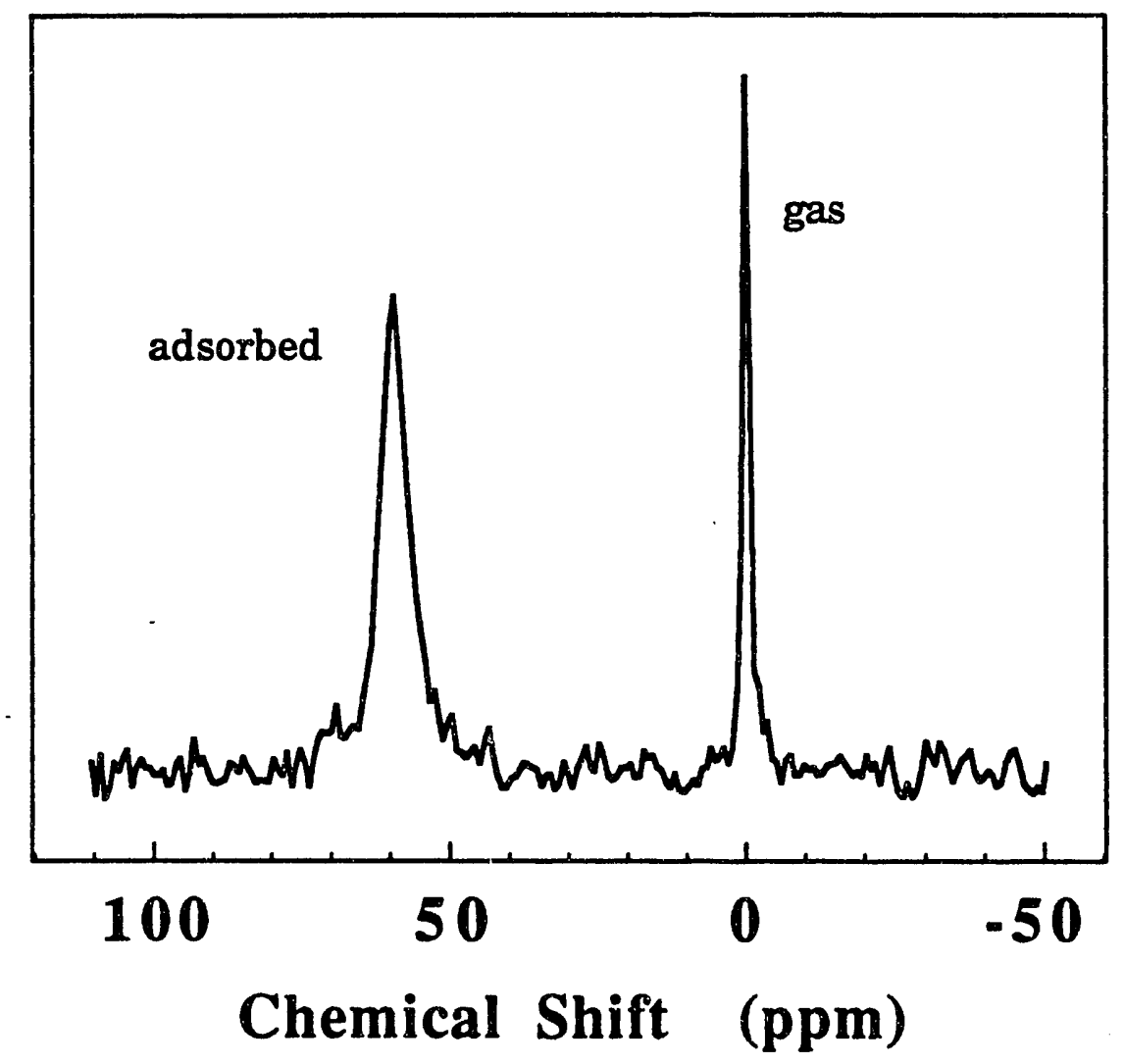

Figure 7.7: Room temperature $(298 \mathrm{~K}){ }^{129} \mathrm{Xe}$ NMR spectra using optically-pumped, natural abundance ${ }^{129} \mathrm{Xe}$ adsorbed onto $\mathrm{Na}-\mathrm{Y}$ zeolite, $\mathrm{P}=16$ torr, chemical shift $=$ $59 \mathrm{ppm}$, line width $=250 \mathrm{~Hz}$. 
is approximately 350 . The reduced enhancement compared to the pure xenon gas experiments is probably due to contaminants which can reduce the pumping efficiency, or relaxation of xenon at the surface, for the case of $\mathrm{Na}-\mathrm{Y}$. However, optical pumping allows us to observe xenon on surfaces where the xenon relaxation time is long (the xenon $T_{1}$ on benzanthracene is many tens of seconds), and signal averaging is impractical.

\subsubsection{Polyacrylic Acid Studies}

A relatively thorough investigation of xenon adsorbed onto polyacrylic acid was carried out using the optical pumping techniques described above. Several conventional NMR studies of xenon adsorbed into polymers (that are discussed in Chapter 3$)$ have been performed recently, $[145,146,147]$ that indicated that xenon could provide interesting information about polymer structure and phase changes. Also, polyacrylic acid seemed a likely candidate for cross-polarization [16] experiments designed to selectively enhance and observe surface nuclei.

Several NMR' spectra of optically pumped ${ }^{129} \mathrm{Xe}$ adsorbed onto polyacrylic acid are shown in Figure 7.8. The spectra are quite different from those of xenon adsorbed onto benzanthracene in two ways. First, at room temperature, there is already a distinct ${ }^{129} \mathrm{Xe}$ peak, at about $3 \mathrm{ppm}$ chemical shift, which we can attribute to xenon atoms which are in contact the polyacrylic acid surface. The line widths of these spectra are also narrower than for xenon adsorbed on benzanthacene. This may be due to a more homogeneous interaction of xenon with the surface of the polymer.

The full data set for xenon adsorbed on polyacrylic acid is shown in Figure 7.9 as a function of temperature and pressure. As the temperature is lowered the xenon chemical shift increases. Even more dramatic is the increased pressure dependence of the chemical shift at the lower temperatures. 

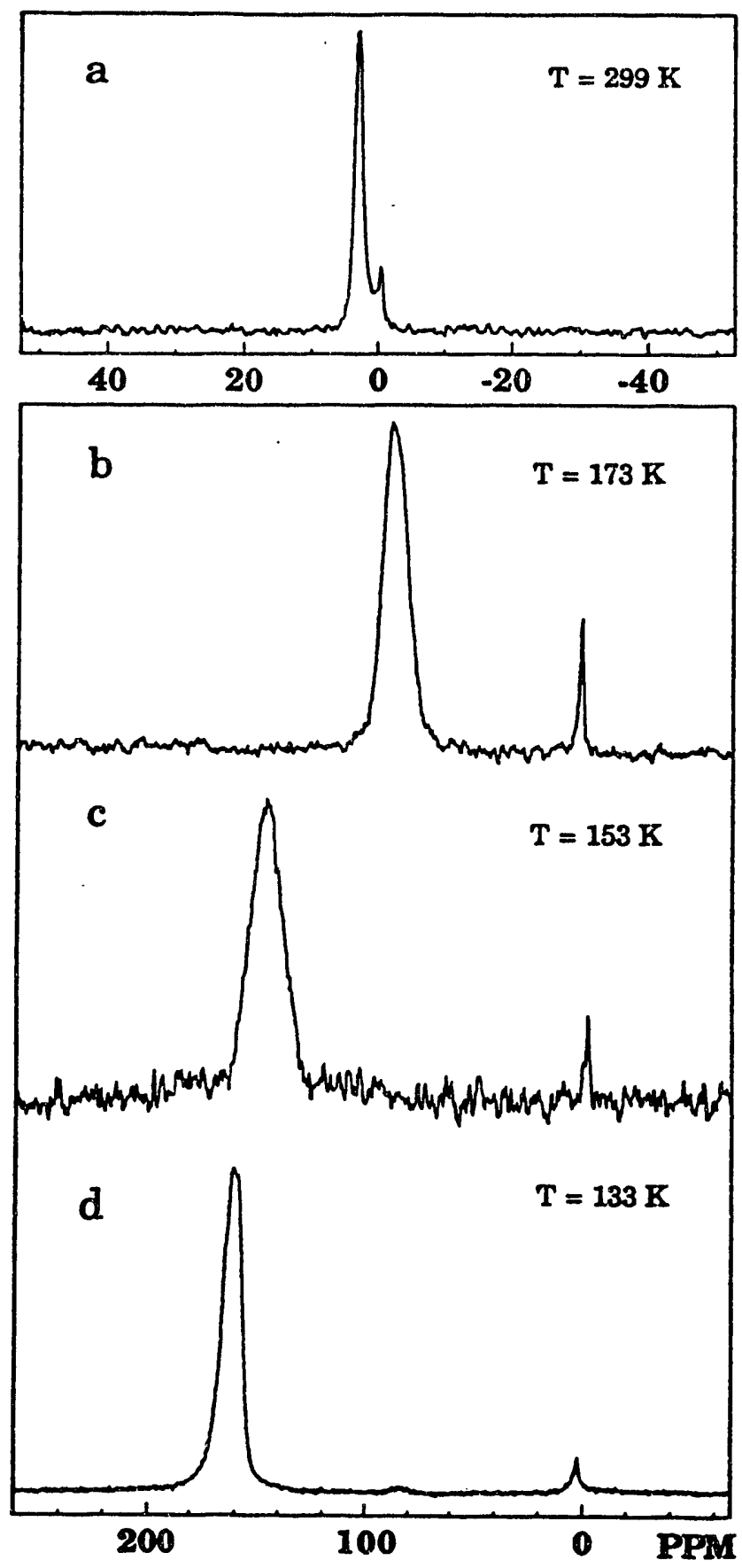

Figure 7.8: ${ }^{129} \mathrm{Xe}$ NMR spectra of xenon adsorbed onto polyacrylic acid at various temperatures: (a) $\mathrm{T}=299 \mathrm{~K}, \mathrm{P}=30$ torr; (b) $\mathrm{T}=173 \mathrm{~K}, \mathrm{P}=20$ torr; (c) $\mathrm{T}=153 \mathrm{~K}, \mathrm{P}=10$ torr; and (d) $\mathrm{T}=123 \mathrm{~K}, \mathrm{P}=2$ torr. 


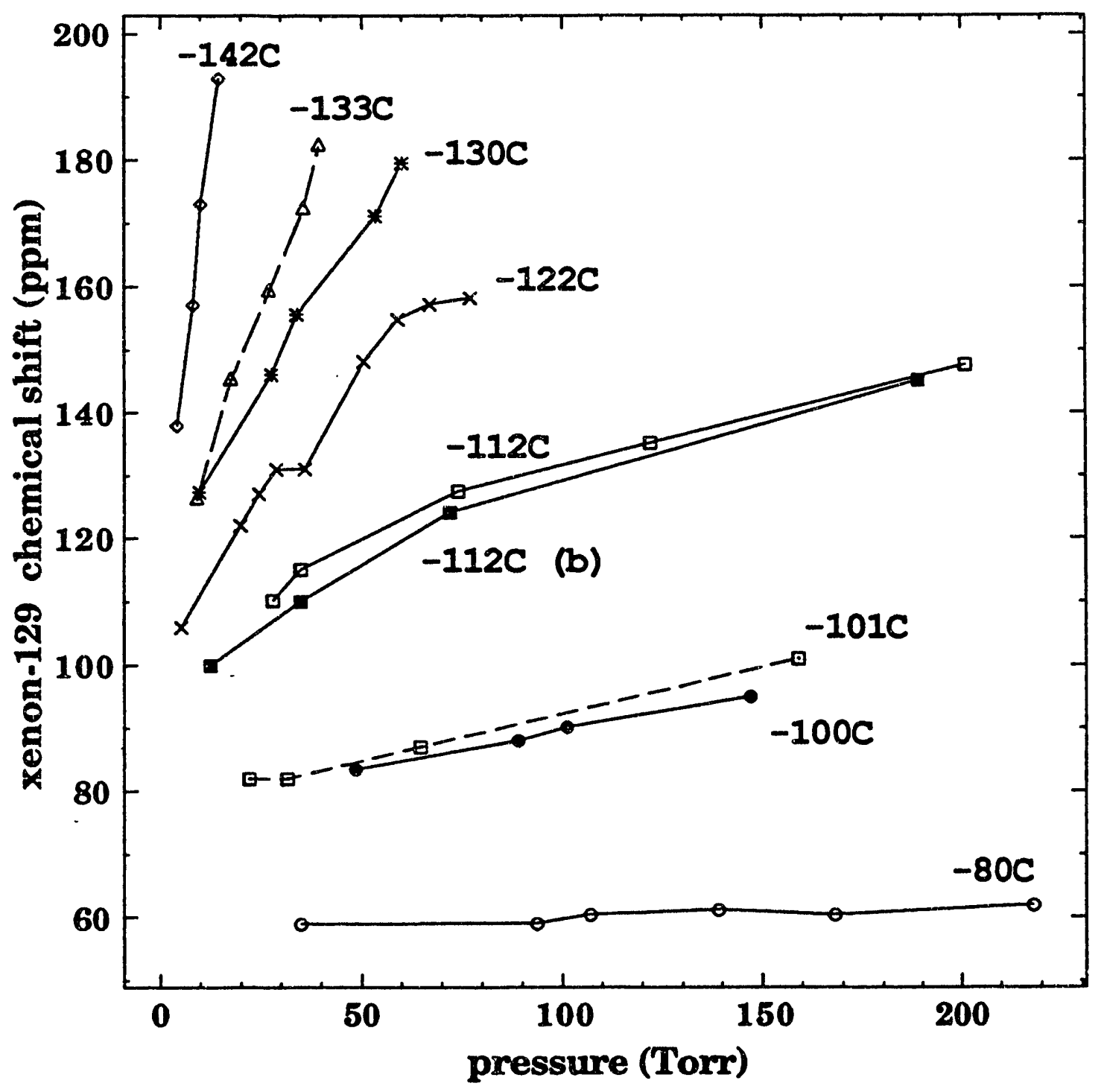

Figure 7.9: Summary of xenon spectra for polyacrylic acid at different temperatures and pressures. 


\subsection{Discussion}

We can understand the large changes of the xenon chemical shift in terms of the xenon sticking time and xenon's diffusion on the surface. The NMR data indicate that there are two regions in the sample volume. First, there is the relatively isolated xenon in the gas phase with a chemical shift of 0 ppm. At room temperature, this is a sizable fraction of the xenon, but as the temperature is lowered, this fraction decreases until about $150 \mathrm{~K}$ where we do not see any gas signal. The other fraction of xenon can contact the surface of the sample. At $298 \mathrm{~K}$ this fraction of xenon is rapidly exchanging between the gas phase and a surface-adsorbed phase, so the measured chemical shift of ${ }^{129} \mathrm{Xe}$ is due to xenon's averaging over the different environments during a time period relevant to the NMR acquisition time (tens of milliseconds). The probability of finding xenon at the surface is obviously much greater the larger the surface area of the material. Thus the reason that room temperature experiments on polyacrylic acid yield a downfield peak separate from the gas peak is primarily due to the fact that the polyacrylic acid sample has a specific surface area of about $15 \mathrm{~m}^{2} / \mathrm{g}$ which is much higher than that for benzanthracene. Second, xenon has a much larger interaction with the polyacrylic acid, as shown by the larger chemical shifts for the polyacrylic sample as the temperature is lowered.

The average sticking time at the surface is given by:

$$
\tau_{s}=\tau_{o} e^{H_{a d s} / k T}
$$

where $\tau_{\circ}$ is the pre-exponential factor which we take to be about $10^{-12} \sec [211]$, and $H_{a d s}$ is the energy of adsorption. Using the adsorption energy derived from the xenon isotherms, which is about $4.2 \mathrm{Kcal} / \mathrm{mole}$, (see Appendix A for details), sticking times are in the range of $10^{-9} \mathrm{sec}$. at room temperature to $10^{-5} \mathrm{sec}$ at $130 \mathrm{~K}$.

Another feature of Figure 7.9 is the pressure dependence of the chemical shift. 
As in the conventional NMR studies of xenon adsorbed onto high surface-area materials such as zeolites [2], the xenon chemical shift is written in terms of a reference frequency $\delta_{0}$, a surface interaction $\delta_{S}$, plus a term which is a linear function of the equilibrium xenon density, $\rho$ :

$$
\delta=\delta_{0}+\delta_{S}+\delta_{(X e-X e)} \rho
$$

The $\delta_{(X e-X e)}$ term is due to the xenon-xenon collisions which occur at or near the surface, and gives rise to the observed slope of the chemical shift as a function of the pressure, or coverage. Fraissard has shown, that because of the rather large adsorption energy in zeolites, the effective pressure of xenon is quite high and therefore there are many xenon-xenon collisions. For polyacrylic acid, the xenon chemical shift is a linear function of the pressure at all temperatures. Therefore only binary collisions are important at the coverages studied. Also, the slope increases dramatically as the temperature is lowered.

To understand this last effect, xenon isotherms were used to convert the temperature and pressure data to xenon coverages at the various temperatures. The three $\mathrm{BET}$ isotherms have an adsorption energy of $4.2 \mathrm{Kcal} / \mathrm{mole}$ and an average surface area of $14.2 \mathrm{~m}^{2} / \mathrm{g}$ (see Appendix A for details), which we can use to compute the xenon coverage by solving for $\theta$ in terms of the pressure and temperature.

The data that are shown in Figure 7.10 cover the range of coverages from 0.03 monolayers of xenon on the surface to about 1.5 monolayers. It is apparent that the dependence of the chemical shift on the coverage is linear, as it was for the pressure dependence. However, the slopes of the lines increase more modestly from about $11 \mathrm{ppm} / \mathrm{monolayer}$ at $-81^{\circ} \mathrm{C}$ to about $70 \mathrm{ppm} /$ monolayer at $-142^{\circ} \mathrm{C}$. The slopes and intercepts of the data in Figure 7.10 can be analyzed in terms of surface residence probability and the Jameson chemical shift ternperature dependences [37], 


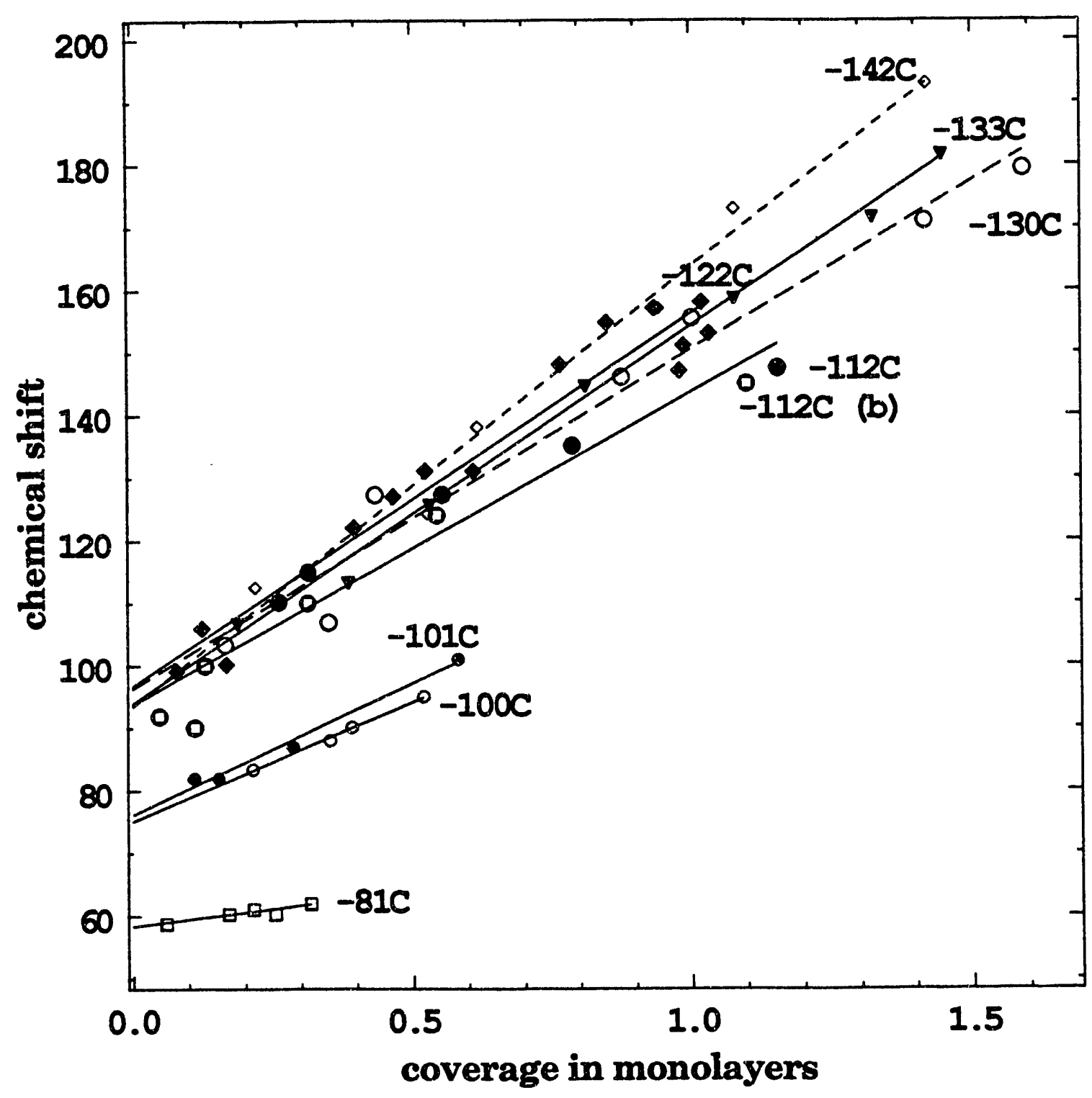

Figure 7.10: ${ }^{129} \mathrm{Xe}$ spectra for xenon adsorbed on polyacrylic acid as a function of the equilibrium temperature and coverage (in monolayers). 
extrapolated to our conditions.

We can model the chemical shift intercepts (that is excluding xenon-xenon interactions for the moment) as a chemical exchange problem such that the chemical shift is due to two parts, the surface value and the gas shift, both of which are weighted by the probability of finding xenon in each phase:

$$
\delta=\delta_{S} P_{S}+\delta_{G} P_{G}
$$

Note that $P_{S}+P_{G}=1$. However, $\delta_{g}$ is defined to be zero, so:

$$
\delta \simeq \delta_{S} P_{S}
$$

The probability of finding a xenon atom at the surface is given by:

$$
P_{S}=\frac{\tau_{S}}{\tau_{S}+\tau_{V}}
$$

where $\tau_{S}$ is the sticking time, defined in equation (7.1) and $\tau_{V}$ is the reciprocal of the collision rate with the surface. Equation (7.5) is not unlike that used by CohenTannoudji [31] for optically pumped mercury relaxation experiments. Normally,

$$
\tau_{V}=\frac{4 \bar{v} S}{V},
$$

where $\mathrm{S} / \mathrm{V}$ is the surface to volume ratio and $\bar{v}$ is the average gas velocity. However, it is not possible to define a surface to volume ratio for a powdered sample such as polyacrylic acid.

We can instead fit the intercepts of the chemical shift in Figure 7.10 to a function of the form of equation (7.4), where $P_{S}$ is given

$$
P_{S}=\frac{\tau_{0} e^{H_{\text {ads }} / k T}}{\tau_{V}+\tau_{\circ} e^{H H_{d d s} / k T}}
$$

by substituting equation (7.1) into equation (7.5). It is not possible to separate the variables $\tau_{\circ}$ and $\tau_{V}$ in the fit. However, with $H_{a d s}=4.2 \mathrm{Kcal} / \mathrm{mole}$, and choosing 
$\tau_{\circ}=10^{-12}$ (a reasonable value), we find that $\delta_{S}=95 \mathrm{ppm}$ and $\tau_{V}=3 \times 10^{-8} \mathrm{sec}$. The chemical shift intercept data and the fit are shown in Figure 7.11. The xenonpolyacrylic acid surface interaction is therefore $95 \mathrm{ppm}$, a value which is somewhat larger than the $86 \mathrm{ppm}$ for xenon in $\mathrm{Na}-\mathrm{Y}$ zeolite measured at $144 \mathrm{~K}$ [95].

A similar analysis of the chemical shift slope data can be made. The slope is an indication of the xenon-xenon interaction at the surface. The probability of finding two xenon atoms at the surface at the same time is $P_{S}^{2}$, and the slope should be related to a temperature dependent surface xenon-xenon interaction $\sigma_{1 s}(\mathrm{~T})$ as:

$$
\frac{d \delta}{d 0}=P_{S}^{2} \sigma_{1 s}(T)
$$

$\sigma_{1 s}(\mathrm{~T})$ should be related to the Jameson second virial coefficient which has been measured in the gas phase at temperatures between $240 \mathrm{~K}$ and $440 \mathrm{~K}$ [37]. Unfortunately, we must extrapolate their data to our temperature region of interest which extends down to about $130 \mathrm{~K}$. In order to relate $\sigma_{1 s}(\mathrm{~T})$ to $\sigma_{1}(\mathrm{~T})$, it will be useful to modify the definition of $\sigma_{1}(\mathrm{~T})$ such that:

$$
\sigma_{1}(T)=\sigma_{1}^{*}(T) \frac{1}{\tau_{c}}
$$

where $1 / \tau_{c}$ is the collision rate of a xenon atom. In this way, $\sigma_{1}^{*}(T)$ is the "second virial coefficient per collision." We do not assume anything about the actual collision parameters, such as duration, which is already contained in the experimentally determined $\sigma_{1}(\mathrm{~T})$. In the gas phase,

$$
\frac{1}{\tau_{c}}=[X e] \sigma_{X e} \bar{v}_{g}=\frac{\bar{v}_{g}^{2}}{3 D_{g}}
$$

where $[\mathrm{Xe}]$ is the xenon concentration, $\bar{v}_{g}$ is the average relative velocity, $\sigma_{X e}$ is the binary collision cross section ( $23 \AA^{2}$ for xenon), and $D_{g}$ is the gas diffusion constant. The average relative velocity is $\bar{v}_{g}=\sqrt{16 k T / \pi m}$ for two xenon atoms. We can 


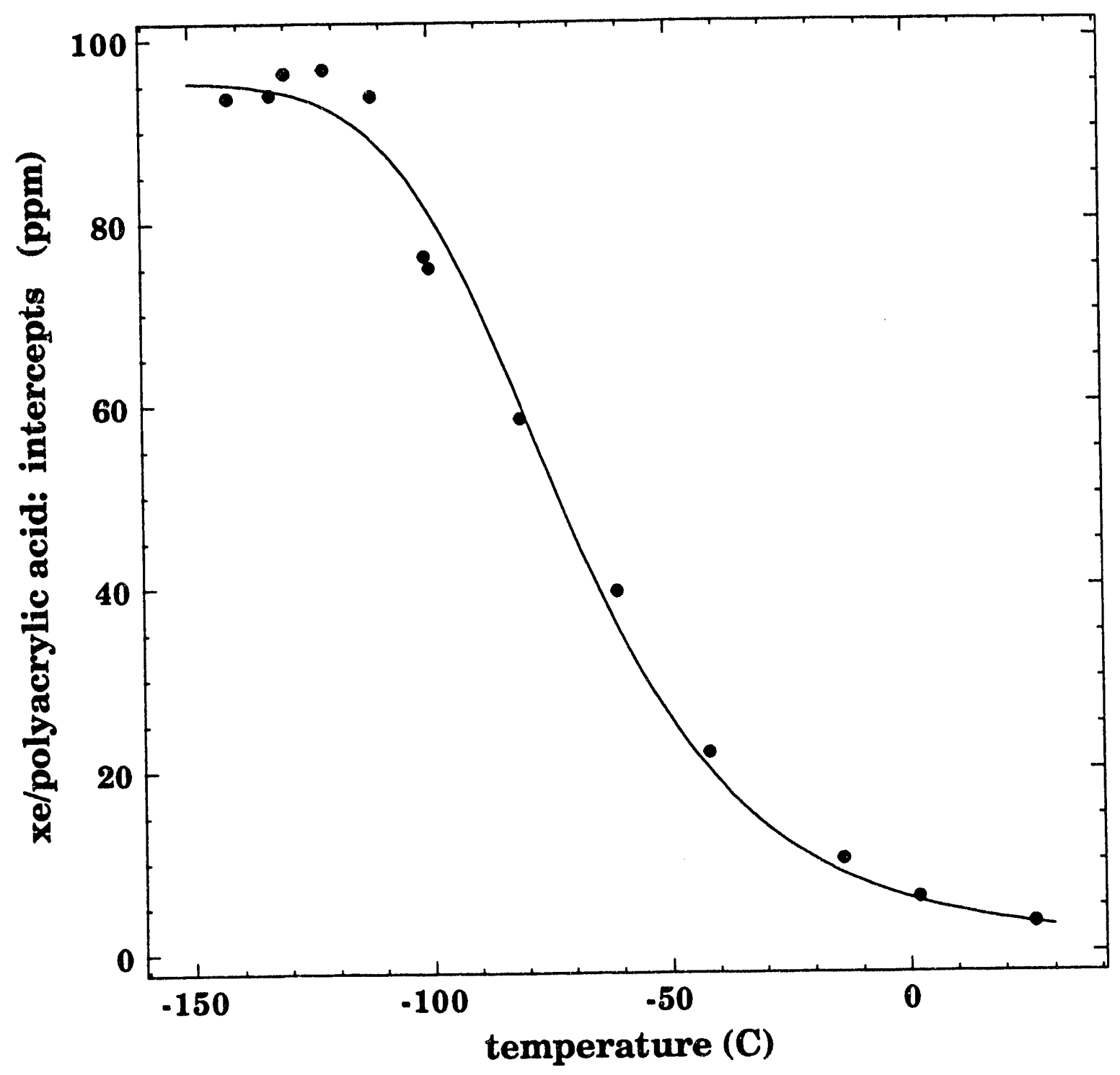

Figure 7.11: ${ }^{129} \mathrm{Xe}$ chemical shift intercepts $(\mathrm{ppm})$ versus temperature and fit as described in the text. 
rewrite equation (7.10) in terms of a surface collision rate, $1 / \tau_{s c}$ :

$$
\frac{1}{\tau_{s c}}=0 \sigma_{s} \bar{v}_{s}=\frac{\bar{v}_{s}^{2}}{2 D_{s}} \text {. }
$$

where $\theta$ is the coverage, $D_{s}$ is the surface diffusion constant, and $\bar{v}_{s}^{2}$ is the surface velocity. The units of $\theta$ and $\sigma_{s}$ are now $\mathrm{cm}^{-2}$ and $\mathrm{cm}$. Since we are talking about two dimensional diffusion, the constant is $1 / 2$ instead of $1 / 3$ as in right side of equation (7.10). An expression for $\sigma_{1 s}(\mathrm{~T})$ may also be written:

$$
\sigma_{1 s}(T)=\sigma_{1 s}^{*}(T) \frac{1}{\tau_{s c}} .
$$

We now make the assumption that the pair-wise, per collision interaction term on the surface, $\sigma_{1 s}^{*}(\mathrm{~T})$, is the same as it is in the gas phase as on the surface:

$$
\sigma_{1 s}^{*}(T)=\sigma_{1}^{*}(T)
$$

In other words, the nature of the pair-wise collisions is not changed by the presence of the surface, but the effect on the chemical shift is dependent on the collision rate which may be very different at the surface. We can therefore relate $\sigma_{1}(\mathrm{~T})$ and $\sigma_{1 s}(\mathrm{~T})$, by first inverting equation (7.9) to get:

$$
\sigma_{1}^{*}(T)=\sigma_{1}(T) \tau_{c},
$$

and using equation (7.13) and equation (7.12) to express $\sigma_{1 s}(\mathrm{~T})$, in terms of $\sigma_{1}(\mathrm{~T})$ :

$$
\sigma_{1 s}(T)=\sigma_{1}(T) \frac{\tau_{c}}{\tau_{s c}} .
$$

Thus we just scale $\sigma_{1}(\mathrm{~T})$ by the ratio of the collision rates in the two phases to obtain $\sigma_{1 s}(\mathrm{~T})$. We now rewrite equation (7.8) using equations (7.10) and (7.11) as:

$$
\frac{d \delta}{d \theta}=P_{S}^{2} \sigma_{1}(T) \frac{\tau_{c}}{\tau_{s c}}=P_{S}^{2} \sigma_{1}(T) \frac{\theta \sigma_{s} \bar{v}_{s}}{[X e] \sigma_{X e} \bar{v}_{g}} .
$$

We can now use equation (7.11) to eliminate $\bar{v}_{s}^{2}$ :

$$
\bar{v}_{s}=2 \sigma_{s} O D_{s}
$$


and so, by substituting for $\bar{v}_{s}^{2}$, we can finally write:

$$
\frac{d \delta}{d \theta}=P_{S}^{2} \sigma_{1}(T) \frac{2 \theta^{2} \sigma_{s}^{2} D_{s}}{[X e] \sigma_{X e} \bar{v}_{g}}
$$

which we can fit to the slopes of Figure 7.10. The coverage $\theta$ in the right side of the equation is a constant, not a variable since $\sigma_{1}(T)$ is determined at a constant pressure. We evaluate the diffusion coefficient at a coverage of 1 monolayer, so $\theta=1 / 23 \AA^{2}$. All of the parameters in equation (7.18) are known, with the exception of $P_{S}$, which is derived from a fit to the chemical shift intercepts, Figure $7.11, \sigma_{1}(T)$ which is extrapolated from the Jameson work, and $D_{s}$ which we would like to determine.

The slope data and fit are shown in Figure 7.12. It can be seen that, although there is quite a lot of scatter in the data, the fit is relatively convincing. The fit must go towards zero at warm temperatures, where the xenon spends little time at the surface and the slope should be just $\sigma_{1}$, the room temperature second virial coefficient, $0.548 \mathrm{ppm} /$ amagat. As seen in the Figure, the fit does go to zero at warm temperatures. Notice that a simple linear fit is not adequate to describe the data. The fit function has no adjustable parameters. Substituting values for $\sigma_{s}(8.8 \AA)$, and $\theta\left(1 / 23 \AA^{2}\right)$, the value of $D_{s}$ is $3.3 \times 10^{-5} \mathrm{~cm}^{2} \mathrm{~s}^{-1}$.

By including a second parameter into the fit, an Arrhenius form

$$
D(T)=D_{\diamond} e^{-E_{d i s s} / k T}
$$

for the temperature dependent diffusion coefficient could be determined. Unfortunately, an attempt to do this yielded a negative energy, indicating increased diffusion as the temperature was lowered. This is probably due to an underestimate for the $\sigma_{1}(T)$ at the lowest temperatures of our study due to the fact that we had to extrapolate the Jameson data over $100{ }^{\circ} \mathrm{C}$. $\mathrm{E}_{\text {diff }}$ is expected to be $10-20 \%$ of the adsorption energy $(4.2 \mathrm{Kcal} / \mathrm{mole}$ for xenon on polyacrylic acid) which would de- 


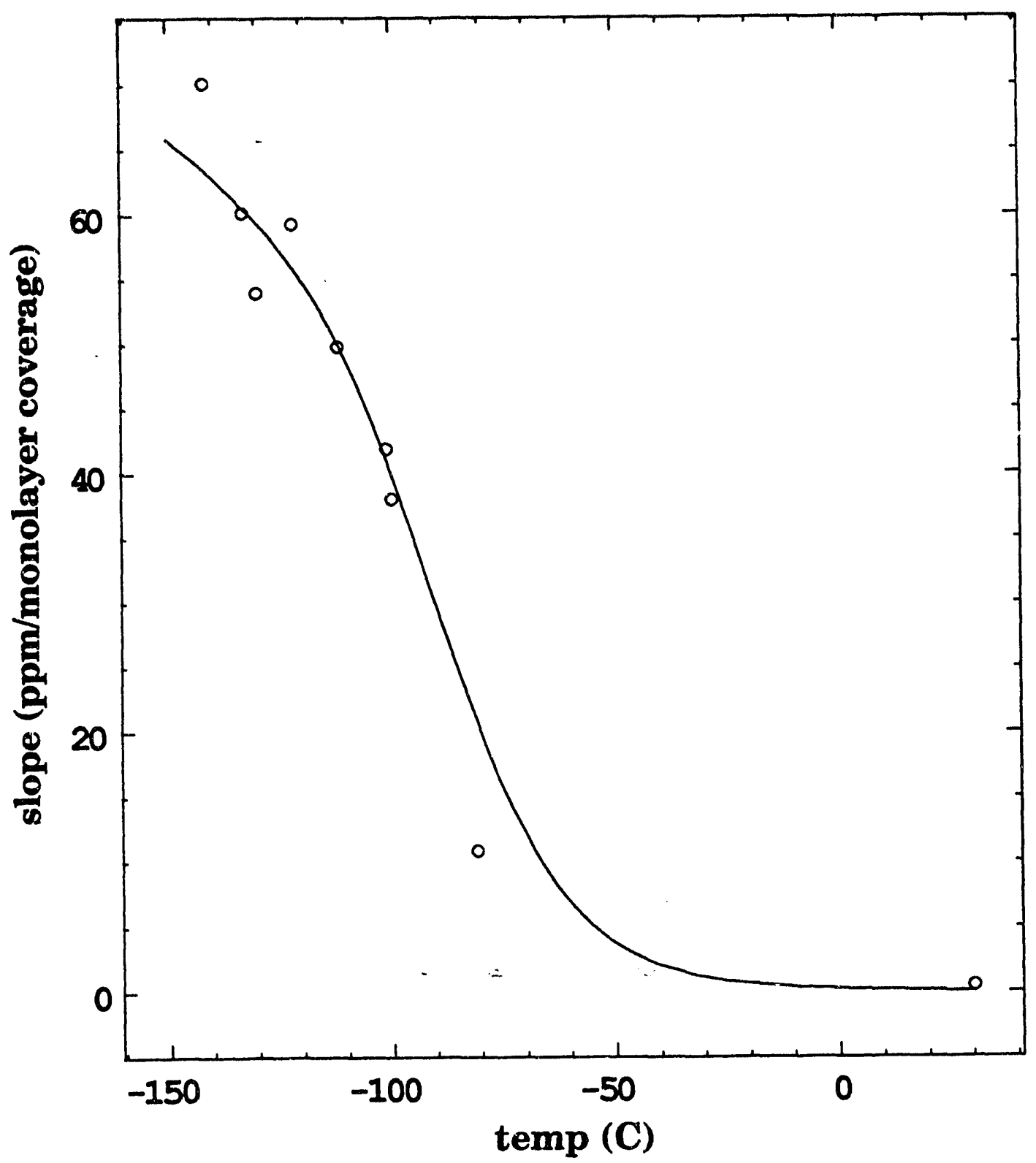

Figure 7.12: ${ }^{129} \mathrm{Xe}$ chemical shift slope (ppm/monolayer) versus temperature and fit using equation 7.18 . 
crease the diffusion constant only by a factor of two over the temperature raisge studied.

The value of the diffusion coefficient fits well with previous studies of ammonia on Vulcan III graphitized carbon black [212]. Their value at $85 \mathrm{~K}$ and a coverage of 0.9 monolayers is $3.5 \pm 0.9 \times 10^{-5} \mathrm{~cm}^{2} \mathrm{~s}^{-1}$, which is not far from our values.

\subsection{Conclusions}

We have demonstrated an enhancement of nuclear spin polarization which extends the possibilities of high field NMR to the study of adsor ,tion on low to moderate surface-area materials. It should be straightforward to increase our polarizations by up to two orders of magnitude using higher power lasers currently available.

Studies on polyacrylic acid indicate that xenon has a rather large interaction with the surface. Some dynamical information is available by analyzing the chemical shift data taken at different temperatures and pressures. An exciting possibility, currently being investigated is the selective enhancement of other surface spins by cross-polarization [16] from optically polarized xenon following adsorption. 


\section{Chapter 8}

\section{NMR of ${ }^{129} \mathrm{Xe}$ Thin Films}

\subsection{Introduction}

A number of techniques have been used recently to investigate thin films, in particular to study properties that differ from those in the bulk material. Opticallydetected ESR has been used, for example, to probe thin semiconductor interfaces [213] and second harmonic generation has been used to examine organic monolayers at the air-water interface [214]. Although NMR is widely used in materials research to study structure and dynamics (over a wide range of timescales), its low sensitive makes it useful primarily for bulk phenomena. A few NMR studies of films have been reported $[67,215]$, mostly adsorbed on high surface-area materials such as exfoliated graphite [69] (Grafoil), with a surface area of about $70 \mathrm{~m}^{2} / \mathrm{g}$. For example, Pettersen and Goodstein attributed the reduction of $T_{1}$ relaxation times in thin layers of methane adsorbed on graphite [215] to the diffusion of methane molecules to paramagnetic impurities on the surface. An intermediate layer of adsorbed xenon interrupted this surface-induced relaxation mechanism to some extent. Neue [67] observed three monolayer films of xenon on graphite and concluded that diffusion to 
surface paramagnetic impurities also played an important in his $T_{1}$ data. His observed line shapes indicated an inhomogeneous diamagnetic interaction of the xenon film with the large magnetic susceptibility of the graphite.

Thin films of helium-three have been studied by Laloë and coworkers [216], who used optical pumping methods to enhance the NMR signal of helium-3 condensed at $400 \mathrm{mK}$ and at a 13 gauss magnetic field. Due to interactions of the nucleus with the large, local-dipolar magnetic field of the highly-polarized film, the resonance frequency of the film depends on its polarization and orientation with respect to the magnetic field:

$$
\omega=\omega_{\circ}+\gamma M_{\circ}\left(3 \cos ^{2} \theta-1\right)
$$

where $\omega_{\circ}$ is the gas frequency, $\gamma$ is the gyromagnetic ratio for helium three, $M_{\circ}$ is the magnetization of the film in gauss, and $\theta$ is the angle between the film and the static magnetic field. The observed spectrum consists of a gas phase signal with two additional singularities for horizontal $(\theta=\pi / 2)$ and vertical $(\theta=0)$ films, consistent with $\mathrm{M}_{0} P_{2}(\cos \theta)$ dependence of the film orientation.

We have employed methods of optical pumping, following Happer and coworkers $[21,78]$, to enhance the NMR signal of ${ }^{129} \mathrm{Xe}$ in high magnetic field [5], allowing us to take advantage of both high. sensitivity and high resolution. We have observed a variety of effects due to film geometry and the temperature in thin (about $1 \mu \mathrm{m}$ ) films of xenon that can be understood in terms of the bulk diamagnetic susceptibility shifts and motional diffusion in the solid. The thinness of the films simplify the spectral line shapes such that there is no radial dependence on the susceptibility. The observed spectra correspond to model magnetic resonance chemical shift anisotropy (CSA) line shapes observed in powdered solids distributed over 1 or 2 angles of orientation with respect to the magnetic field. In addition to its effects on the spectra of film and bulk spectra, bulk diamagnetic susceptibility can play an important role 
in magnetic resonance imaging experiments, for example, where the orientation or geometry of the sample volume can affect the resonance frequencies [217].

\subsection{Theory}

The magnetic susceptibility arises from the second-order energy correction for electronic states in a magnetic field $[6,218]$, and can be written:

$$
\chi=\chi_{D}+\chi_{P}=\frac{-e^{2}}{6 m c^{2}} \sum_{i}\left\langle 0\left|r_{i}^{2}\right| 0\right\rangle+\chi^{H F}
$$

where $\chi_{D}$ and $\chi_{P}$ are the diamagnetic and paramagnetic contributions to the susceptibility, and $\chi^{H F}$ are the so-called high frequency terms. In atomic xenon there is no paramagnetic term where, in the usual case, the nucleus is chosen as the origin of the vector potential [218]. The bulk susceptibility is related in form to a more commonly observed resonance shift, the chemical shielding, which also has diamagnetic and paramagnetic terms, but whose expectation values are multiplied by a factor of $1 / r^{3}$ [13]. As a result, the chemical shielding is sensitive to shorter-range perturbations of the electron clouds such as chemical bonding.

The susceptibility for a cylindrically-shaped annular sample has been analyzed by Zimmerman and Forster [219] in which they considered a sample consisting of concentric layers of materials (sample regions plus glass tubes) with different susceptibilities. The relative change of the magnetic field in a sample region $i$ due to the susceptibility is given by the following expression $[219,220]$ :

$$
H_{i} / H_{\circ}=\left[1-\frac{1}{2}\left(\mu_{i}-\mu_{\text {outer }}\right)\right]-\frac{1}{2} \sum_{j=1}^{j=i-1} a_{j}^{2}\left(\mu_{j+1}-\mu_{j}\right) \cos 2 \theta / r^{2}
$$

where $\mu_{i}$ are the permeabilities of the various concentric regions and $\cos 2 \theta$ describes the angular dependence of the anisotropic bulk magnetic susceptibility in a magnetic 
field. For our case, $i=3, \mu_{1}=\mu_{2}=1$ (ie. vacuum), and $\mu_{\text {outer }}=\mu_{\text {air }}$ so

$$
H_{3} / H_{\circ}=\left[1-\frac{1}{2}\left(\mu_{3}-\mu_{\text {air }}\right)\right]-\frac{1}{2} a_{2}^{2}\left(\mu_{3}-1\right) \cos 2 \theta / r^{2} .
$$

The first term is an isotropic shift, and the second can be expressed in terms of susceptibility, by using rationalized SI units, $\mu=1+\chi_{V}$. The maximum and minimum values observed are at $\theta=0$ and $90^{\circ}$ respectively, and the difference is just

$$
\Delta H_{3} / H_{\circ}=\left(a_{2} / r\right)^{2} \chi_{3}
$$

which, for a thin film where $r \simeq a_{2}$, simplifies to $\chi_{3}$. A simple case can be seen in Figure 2.2. A measure of the thinness of these films is the almost vertical increase of the signal intensity at the peaks of the spectrum for the cylindrical sample. As the thickness of the film is increased, such that the ratio of the inner and outer radii of the cylindrical film:

$$
\left(\frac{r_{\text {inner }}}{r_{\text {outer }}}\right)^{2}<<1,
$$

the singularities of the spectrum start to show broadening [219] which is visible in the spectra of Chu et al. [217] for coaxial water samples.

The line shapes for our films may be derived following Haeberlen [221] who considered the related problem of spectral line shapes for a distribution of orientations of crystallites with a chemical shift anisotropy. The angular dependence of the magnetic susceptibility can be described by a $\cos 2 \theta$ dependence where $\theta$ is the angle between the film normal and the magnetic field. The line shape for an arbitrary sample geometry may be complicated, as in the case of a general solid ellipsoid with axes $\mathrm{a} \neq \mathrm{b} \neq \mathrm{c}$. However, for the symmetric cases, as illustrated by this work, the line shapes simplify considerably. The frequency components of the spectrum can be written in terms of the film orientation and the bulk magnetic susceptibility:

$$
\omega=\omega_{\circ}\left[1+\sigma+\delta+\frac{1}{2} \chi \cos 20\right]
$$


where $\sigma$ is the chemical shift (which may be temperature dependent, as in the case of solid xenon for example), $\delta$ is the isotropic shift, and $w_{0}$ is the reference frequency. The intensity of the spectrum may be written, integrated over all angles as:

$$
\int I(\omega) d \omega=\iint d A(\theta, \phi)
$$

In order to express the area element, $\mathrm{dA}$ (which is $\ell d 0$ for the cylindrical distribution) in terms of the frequency, we want to find $d \theta$ in terms of $d \omega[217,219,140]$. Inverting equation 8.7:

$$
\theta=\frac{1}{2} \arccos (2 \Delta \omega / \Delta \chi)
$$

where $\Delta \omega=\frac{\omega}{\omega_{0}}-(1-\sigma-\delta)$, and differentiating,

$$
d \theta=\frac{1}{\Delta \chi} \frac{d \omega}{\sqrt{1-(2 \Delta \omega / \Delta \chi)^{2}}} .
$$

We can see that from equation (8.8), this is the line shape for the cylindrical sample cell. The two singularities are at $\Delta \omega= \pm \frac{1}{2} \Delta \chi$. Incidentally, this is the same line shape which is expected for an axially symmetric two dimensional chemical shift pattern in a powder where there is an isotropic distribution of orientations along one axis of rotation $(\theta)$ with respect to the magnetic field. This is case for an oriented polymer sample perpendicular to the magnetic field [140, 223]. However, in our experiments on xenon films, we observe the shifts due to the bulk susceptibility $\chi$, and not $\sigma$.

For the spherical film geometry, we can again write the frequency dependence of the film's orientation as in equation (8.7). For the sphere, the elemental area $\mathrm{dA}$ is $\sin \theta d \theta$. Substituting $2 \cos ^{2} \theta-1$ for $\cos 2 \theta$, and inverting equation (8.7) as before:

$$
\cos 0=\frac{1}{\sqrt{2}} \sqrt{1+2 \Delta \omega / \Delta \chi}
$$

where again, $\Delta \omega=\frac{\omega}{\omega_{0}}-(1-\sigma-\delta)$. Differentiating, gives

$$
\sin \theta d \theta=-\frac{1}{\sqrt{2}} \frac{d \omega}{\sqrt{1+2 \Delta \omega / \Delta \chi}}
$$


with a singularity at $\Delta \omega=\frac{1}{2} \Delta \chi$. The width of the spectrum is $\Delta \chi$. A similar problem of describing the line shape for a distribution of molecular crystallites due to the anisotropic chemical shift was first considered by Bloembergen and Rowland [224] (see also Haeberlen [221] and Mehring [140]). However, we are considering the macroscopic distribution of a film in the magnetic field.

\subsection{Experimental}

The experimental methods have been described previously [5]. Briefly, the xenon is polarized in low magnetic field in the fringe magnetic field $(250 \mathrm{G})$ underneath the bore of a superconducting NMR magnet. We optically-pump rubidium vapor to approximately $100 \%$ using circularly-polarized infrared laser light. The ${ }^{129} \mathrm{Xe}$ nuclei are polarized via spin-exchange van der Waals collisions with rubidium atoms. After pumping for about $30 \mathrm{~min}$, the polarized xenon is transported to the NMR sample region and frozen at temperatures below $150 \mathrm{~K}$ onto the surface of NMR sample cells with various geometries. The sample cells fit reasonably tightly into the rf coil, which is approximately $1 \mathrm{~cm}$ in diameter and $2 \mathrm{~cm}$ long. The sample region is cooled with cold flowing nitrogen gas and is temperature regulated using a nichrome wire heater to $\pm 1{ }^{\circ} \mathrm{C}$. Spectra are obtained by Fourier transformation of the NMR signal acquired following a radio frequency pulse at $51.4 \mathrm{MHz}$. For the spin-echo experiments described below, a Carr-Purcell sequence [225] consisting of evenlyspaced $180^{\circ}$ pulses applied after a single $90^{\circ}$ pulse which refocuses the inhomogeneous decay of the magnetization. In general, spectra were obtained immediately after introducing the xenon into the sample regions. However, for the order to perform experiments in the capillary tube, the temperature was dropped from slightly above the freezing temperature ( $145 \mathrm{~K}$ for a xenon pressure of 220 torr) to $10^{\circ} \mathrm{C}$ colder to 
prevent xenon from freezing outside the sample region.

\subsection{Results}

Spectra are shown in Figure 8.1 for samples of xenon in several differently shaped NMR sample cells: sphere, cylinder, and flat rectangular boxes with planes perpendicular and parallel to $B_{0}$. Schematic figures are shown next to each spectrum, illustrating the sample cell geometry.

Figure 8.2 shows the difference in the spectra from the thin film cylindrical sample [Figure 8.2(a)] versus a bulk cylindrical solid formed in a capillary tube [Figure 8.2(b)] for sample cells with the same geometry. The bulk signal comes from a capillary tube $250 \mu \mathrm{m}$ in diameter, and the xenon freezes relatively uniformly throughout the volume, as indicated by the symmetric line shape and uniform isotropic shift of $\frac{1}{3} \chi$ as expected in Figure 8.2(b). There is a fair amount of residual broadening, however.

In Figure 8.3 spectra for spherically-shaped films are shown at $150 \mathrm{~K}$ and $123 \mathrm{~K}$. At the lower temperature, dipole-dipole interactions cause a homogeneous broadening of the line as seen in Figure 8.3(a). At the higher temperature, the spectral features of the spectrum are sharp because diffusion in the solid averages out the dipole-dipole coupling that normally leads to line broadening. The isotropic chemical shift is also lower as discussed below. Carr-Purcell spin-echo experiments [225] show that the homogeneous line width is only $10 \mathrm{~Hz}$ at $150 \mathrm{~K}$, in accordance with the results of Yen and Norberg [56] (corrected for isotopic abundance) who studied the effects of diffusion in bulk solid xenon on the line width. In Figure 8.3(a) the spectrum for the spherical film was fit to a function of the form of equation 8.12 (dotted line) which gives $\Delta \chi=13.5 \mathrm{ppm}$ and a residual Gaussian broadening of $25 \mathrm{~Hz}$. The value of the cgs molar bulk magnetic susceptibility is $-43.9 \times 10^{6}[71,227]$, in agreement 

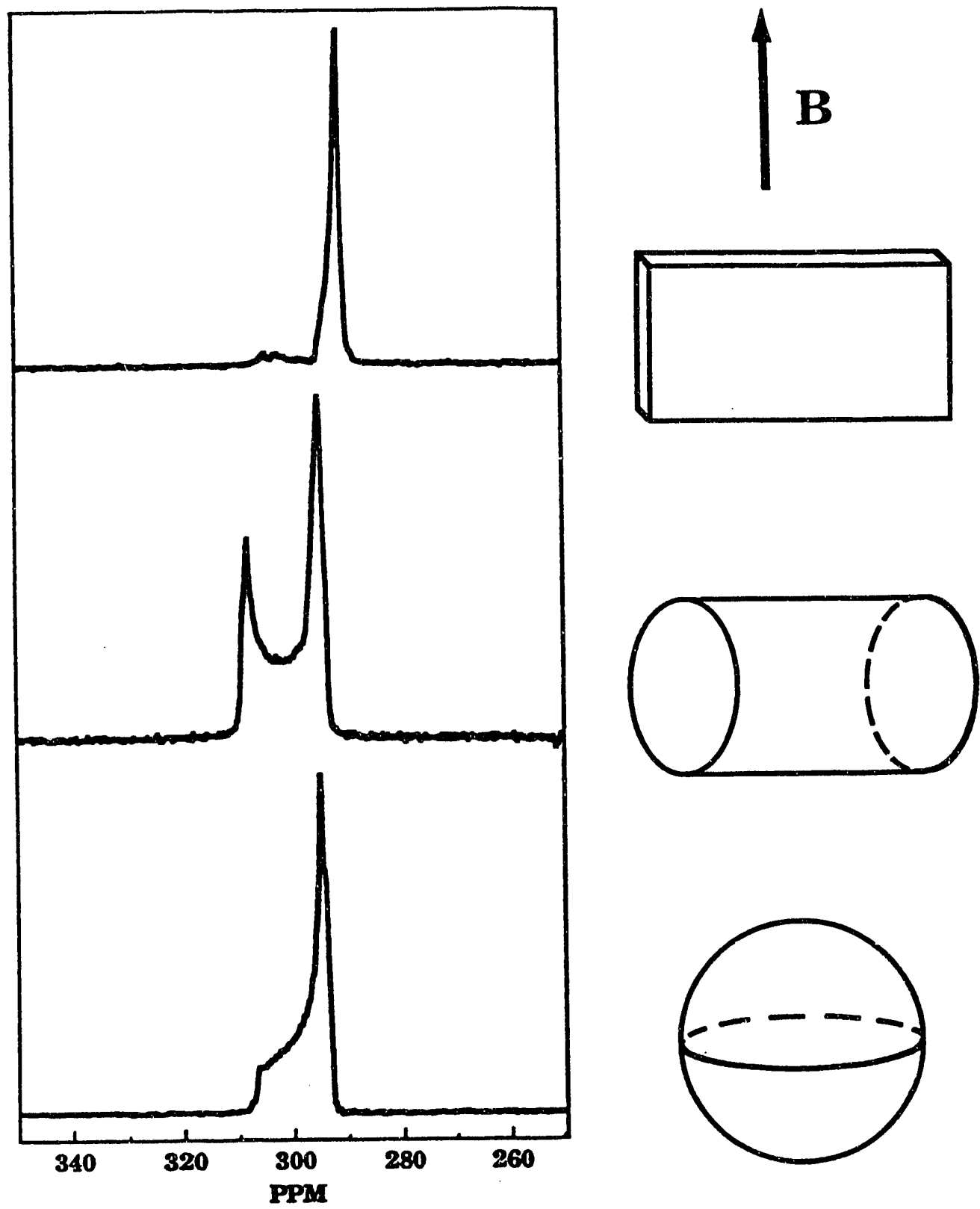

Figure 8.1: ${ }^{129} \mathrm{Xe}$ NMR spectra for xenon thin films frozen on the surfaces of different geometrically shaped NMR sample cells: (a) sphere (radius $0.6 \mathrm{~cm}$ ), (b) cylinder (radius $0.5 \mathrm{~cm}$, perpendicular to $\mathrm{B}_{\circ}$ ), flat rectangular box $(3.0 \mathrm{~cm} \times 1.0 \mathrm{~cm} \times 0.1 \mathrm{~cm}$ ) with planes parallel (c), and perpendicular (d) to $B_{\circ}$. A schematic figure is shown to the right of each spectrum corresponding to its respective film geometry. 


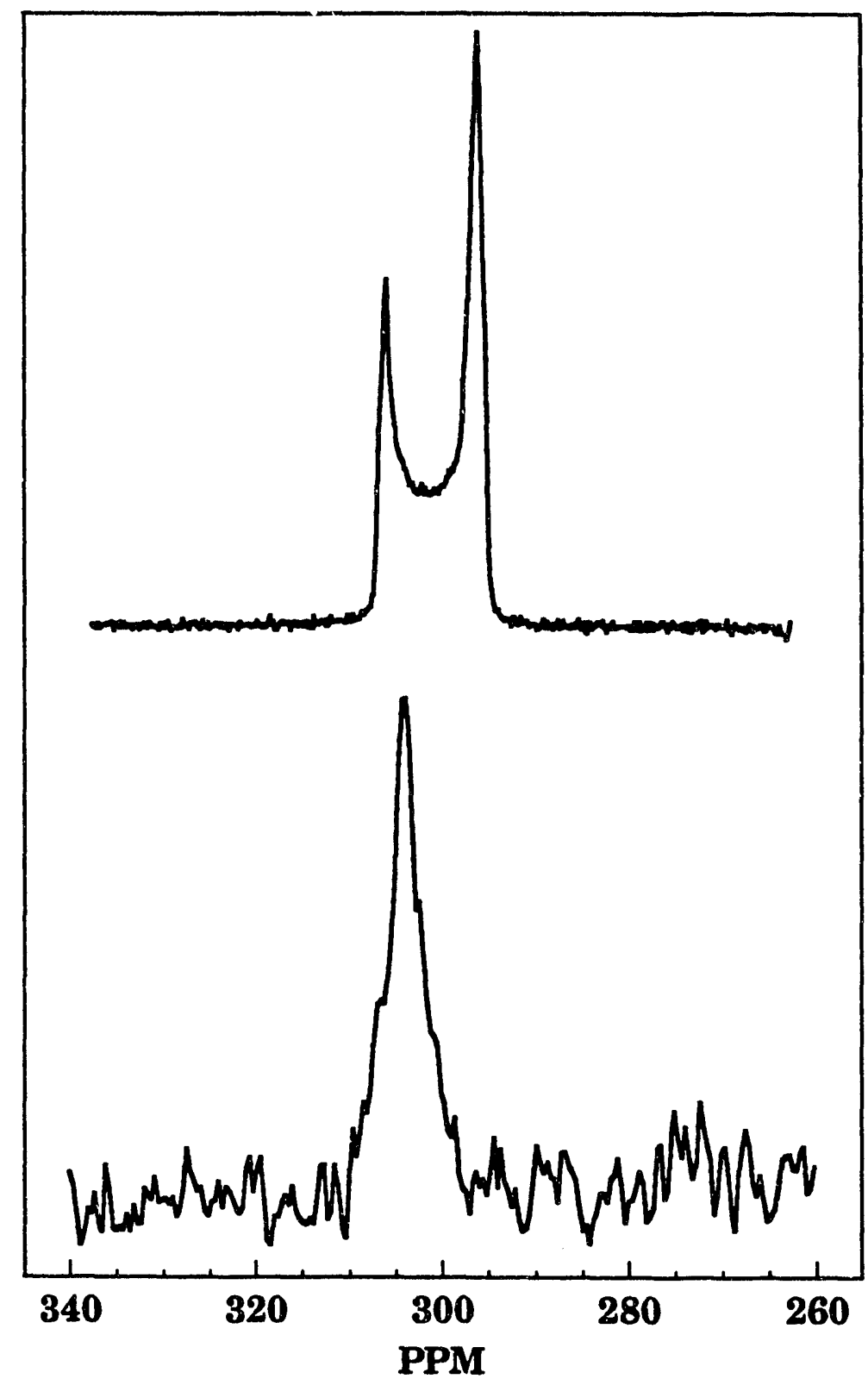

Figure 8.2: The spectrum for the cylindrical sample thin film shows two sharp singularities in (a) while only the isotropic (average) value is observed in the capillary tube where bulk xenon is detected in (b). Residual susceptibility effects broaden the line by about $150 \mathrm{~Hz}$. 

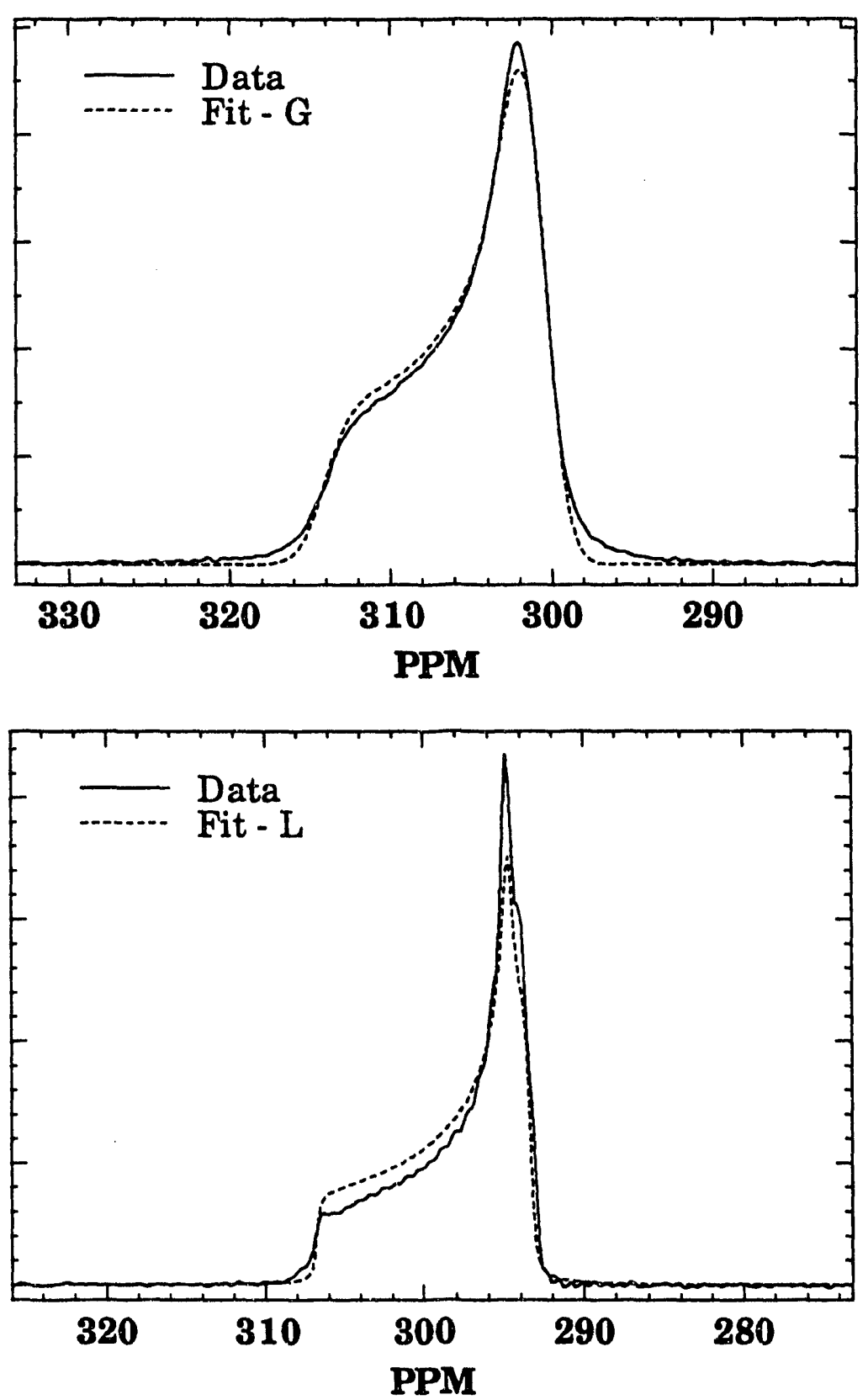

Figure 8.3: Spectra of xenon thin films in the spherical sample cell at different temperatures show the effects of diffusion of the xenon in the film, and the effect on the chemical shift of the line. (a) $\mathrm{T}=140 \mathrm{~K}$, the residual line width is only $25 \mathrm{~Hz}$ due to the averaging by diffusion of dipole-dipole couplings in the xenon solid. The chemical shift of the singularity is $295 \mathrm{ppm}$. (b) At $\mathrm{T}=123 \mathrm{~K}$, the residual line width is now $160 \mathrm{~Hz}$, close to the rigid lattice line width. The chemical shift of the singularity is $303 \mathrm{ppm}$. 
with calculations [226], which can be converted to a volume (rationalized SI units) susceptibility by the following expression (in ppm) [217]:

$$
\chi_{V}=4 \pi \chi_{M} \frac{\rho}{M} \times 10^{6}
$$

where $\rho$ is the density and $M$ is the mass. $\chi_{V}$ for xenon is $-14.8 \mathrm{ppm}$, which is in fair agreement with the observed splitting in the cylindrical sample [Figure 8.1(b)] of $15.1 \mathrm{ppm}$. The difference is probably due to the susceptibility of the glass.

We also investigated the temperature dependence of the chemical shift of the solid for the flat rectangular sample cell to avoid isotropic susceptibility shifts. The data from our experiments are shown in Figure 8.4 along with isotropic chemical shift data of solid xenon from references [56] and [57]. The temperature dependence is linear with a slope of $-0.278 \mathrm{ppm} /{ }^{\circ} \mathrm{C}$. Converting to density using the data from Packard and Swenson [228], we find the density dependence to be $0.575 \mathrm{ppm} /$ amagat, in agreement with the work of Carr and Brinkmann [57, 229]. We also performed a few experiments using the cylindrical sample cell at different temperatures, and found no observable temperature dependence of $\chi_{D}$, as expected.

\subsection{Discussion}

The line shape for the spherical cell showed a slow time dependence that was probably due to temperature gradients which caused a slow, macroscopic migration of the film towards the coldest part of the sample cells. In the spherical cell, the low field (higher resonance frequency) part of the spectrum was observed to decrease in intensity. In the cylindrical cell, the time dependence was opposite, with the line shape changing to enhance the low field part of the spectrum. These effects are probably not due to the influence of gravity (as in the case of liquid helium films [216]), which would cause a drift to higher resonance frequency in all of our samples. 


\section{Xe Solid}

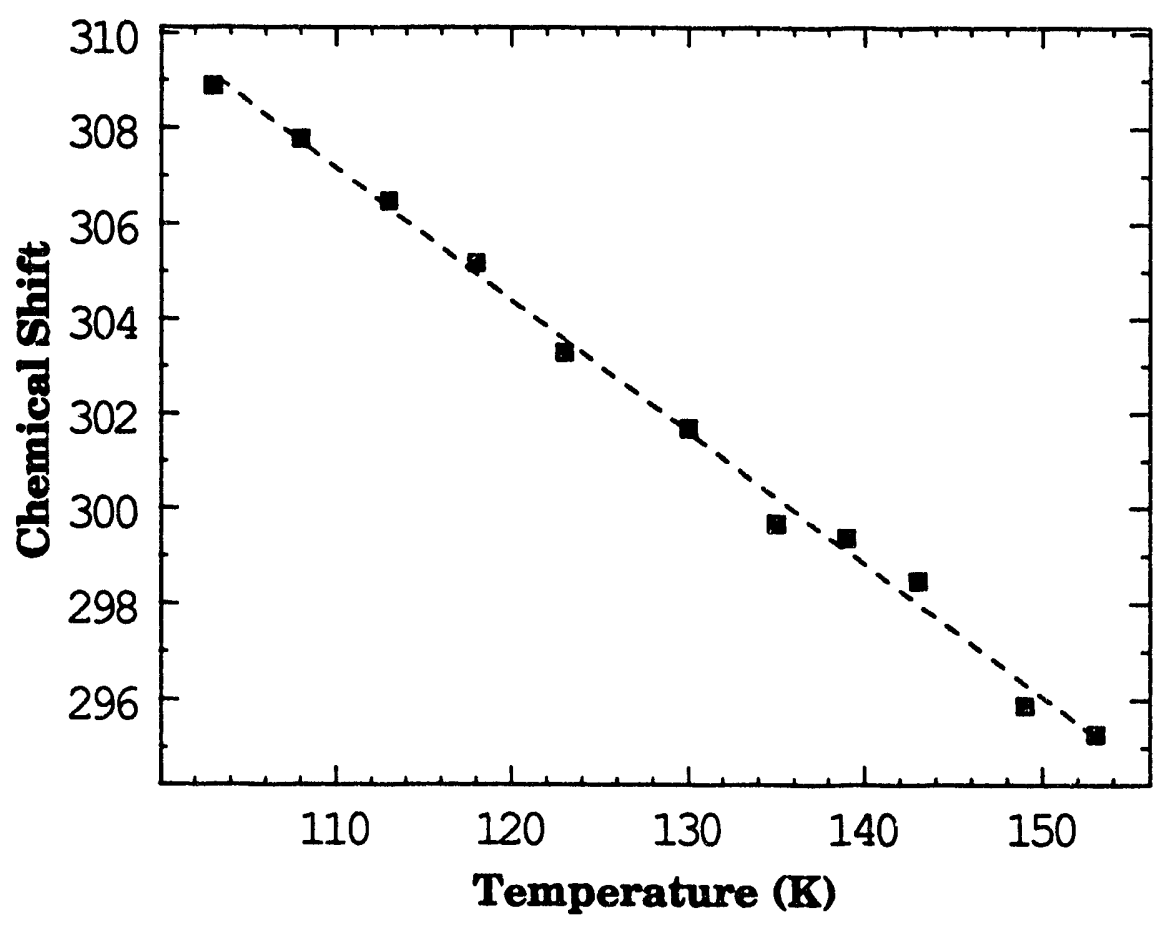

Figure 8.4: Temperature dependence of the chemical shift for the rectangular sample cell (parallel to $\mathrm{B}_{\mathrm{o}}$ ). The slope of the line is $-0.278 \mathrm{ppm} /{ }^{\circ} \mathrm{C}$, in agreement with reference [56]. 
The residual broadening of the spectrum for the spherical sample at $150 \mathrm{~K}$ is somewhat large compared to the expected value from Yen and Norberg [56]. At such temperatures, diffusion is present in the solid which has the effect of narrowing the line width. However, spin echo experiments showed that the observed broadening was inhomogeneous in nature and probably due to residual magnetic field inhomogeneities over the sample volume. The fits to the line shapes also indicated a slightly non-ideal film formation. Residual susceptibility effects can account for the residual line widths in the flat cells and the capillary tube. The homogeneous line width derived from the echo experiments was $10 \mathrm{~Hz}$. At lower temperatures, the xenon spectrum observed has the expected rigid lattice line width of $570 \mathrm{~Hz}$ as expected from reference [56], corrected for our higher ${ }^{129} \mathrm{Xe}$ isotopic abundance.

\subsection{Conclusions}

In summary, optical pumping techniques allow the observation of ${ }^{129} \mathrm{Xe} \mathrm{NMR}$ in xenon thin films. Bulk diamagnetic susceptibility effects are easily seen in these samples and can be understood in terms of line shapes corresponding to different distributions of orientations of the films with respect to the applied magnetic field. Effects of temperature on the chemical shift $\sigma$ and the line broadening are readily observed in the spectra. 


\section{Appendix A}

\section{Isotherms}

\section{A.1 Introduction}

Isotherm data are an important aspect of the surface studies presented in the preceding chapters for they are used to determine the surface areas of the powdered samples and the adsorption energy of xenon on these material surfaces. There are several models that describe the surface adsorption of gases reasonably well that depend on the characteristics of the particular gas and surface involved [230]. In the case of strong adsorption sites, and single layer adsorption, the Langmuir isotherm provides a good description of the adsorption behavior [211]. In the surfaces we have studied, xenon does not have a particularly strong, site specific interaction with the surface. In materials such as $\mathrm{Na}-\mathrm{Y}$ zeolite, xenon is thought to spend most of its time at the surface, but it probably migrates quickly throughout the zeolite structure. Indeed, over the tirne scale relevant to an NMR experiment (tens of milliseconds), xenon can difiuse throughout an entire $\mathrm{Na}-\mathrm{Y}$ zeolite crystallite [115] which is of the order of $1 \mu \mathrm{m}$ in diameter. In such a case, the BET (Brunauer, Emmett, and Teller [231]) isotherm is thought to provide a reasonably good model. According 
to the BET theory, an adsorbing gas such as xenon or nitrogen has an adsorption energy associated with the first monolayer formed, and another, smaller adsorption energy for further layers which adsorb. The theory allows for the possibility of the formation of small multilayer islands of adsorbed atoms that are in competition with the completion of entire monolayers due to entropy considerations. The equation for a BET isotherm is:

$$
V=\frac{V_{\text {mon }} c x}{(1-x)[1+x(c-1)]}
$$

where $\mathrm{V}$ is the volume of adsorbed gas at STP, $x=p / p_{\circ}$ is the reduced pressure, and $V_{\text {mon }}$ is the volume that an amount of adsorbing gas equal to that which forms a monolayer on 1 gram of sample would occupy at STP. The quantity $c$ is related to the surface adsorption energy by:

$$
\ln c=E_{\text {sur } \delta}-E_{\ell}
$$

where $E_{\ell}$ is the heat of liquification of the adsorbate.

\section{A.2 Gas Rack}

Isotherm data were collected on a glass vacuum rack using a silicone oil diffusion pump capable of pressures below $10^{-5}$ torr. A diaphragm pressure gauge (MKS company) was used to measure the equilibrium pressure during the isotherm. Figure A.1 is a schematic representation of the relevant parts of the gas rack used in the measurements. Starting with a known volume $V_{\circ}$ of helium gas in the bulb, the other volumes may be determined by expanding the helium into them one by one at constant temperature, measuring the initial and resulting pressures and calculating the added volumes. For instance, solving for $V_{1}$ in the following equation:

$$
P_{0} V_{0}=P_{1}\left(V_{0}+V_{1}\right)
$$




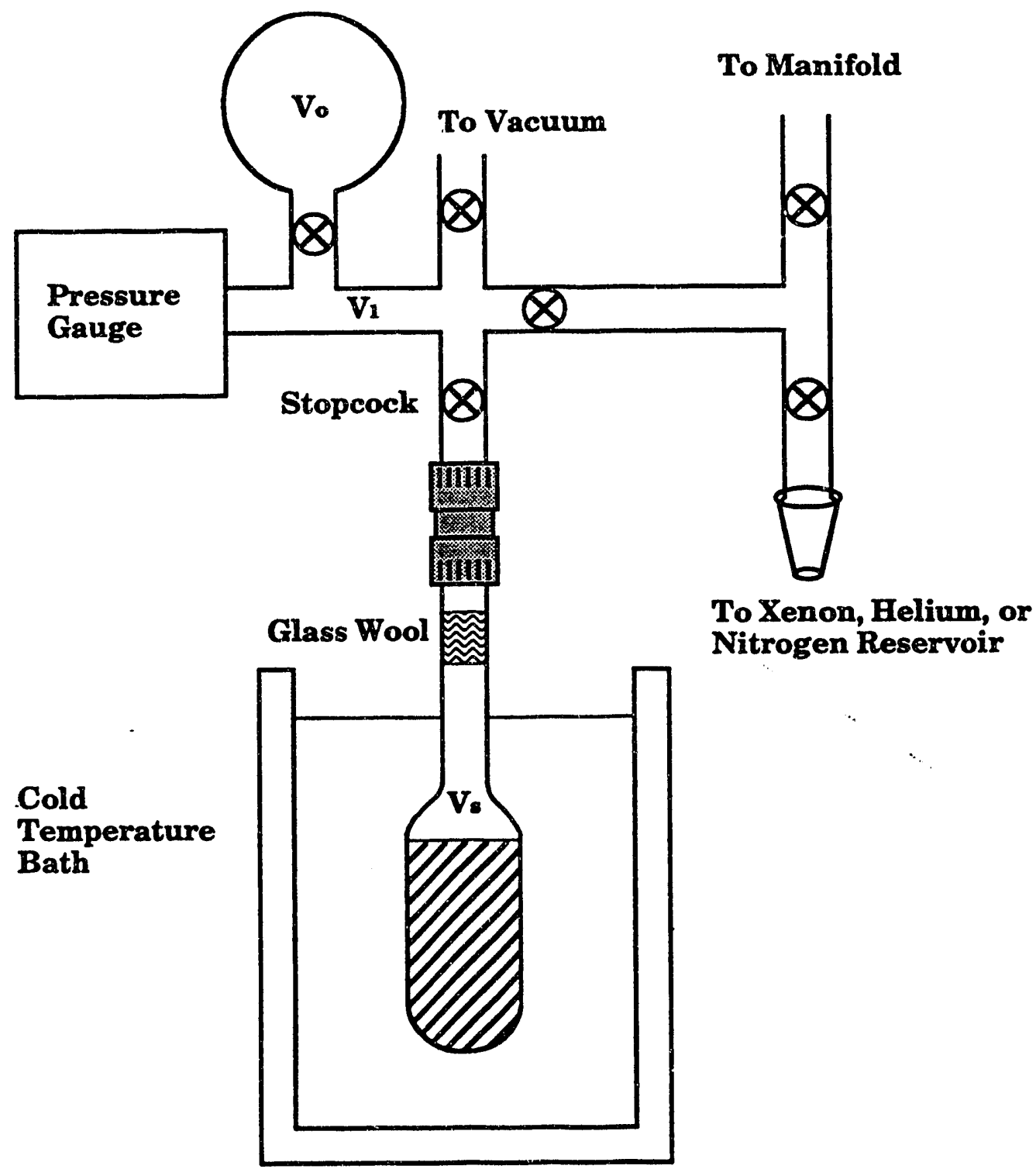

Figure A.1: Schematic diagram of the relevant parts of the gas rack used in isotherm measurements. See text for explanation. 
gives the volume of the section marked $V_{1}$ in the Figure. Typical values for the gas rack used were: $V_{\circ}=55.76, V_{1}=25.1$, and $V_{s}=4.8$. The dead volume of the sample is the critical quantity to measure for accurate BET surface area and adsorption energy determinations.

To perform the BET measurement, initial and final pressures are recorded starting with an initial pressure $P_{i}$ in volume $V_{1}$ and expanding it into the sample region $V_{1}+V_{s}$. The adsorbed volume of gas is given by:

$$
V_{a d s}=P_{f}\left(V_{f}-V_{i}\right)-P_{i} V_{i}
$$

This method was used in the determination of the isotherms in Chapter 4 for xenon adsorbed onto the $\mathrm{Na}-\mathrm{Y}$ zeolite with organic guest samples.

For isotherms conducted at low temperatures (such as for the samples in Chapter 7), cold temperature baths such as liquid nitrogen for the nitrogen isotherms and liquid nitrogen/acetone for the xenon measurements are used. There are two methods of correcting for the low temperature of the sample region. One method is to calculated all temperatures and pressures in terms of STP conditions. Unfortunately, the sample dead volume is often overestimated, for not all of the sample volume is at the cold temperature of the surface material.

Another, less rigorous, but perhaps more accurate method, is to use the dead volume measurement at room temperature with the sample at the isotherm temperature. This way the helium gas experiences the two regions as they will be during the actual isotherm measurement, and the correction is made of automatically. This method is used in Fraissard's laboratory in Paris and seems to give good results. A final temperature correction to STP is made once $V_{\text {mon }}$ has been calculated, to compute the correct surface area. 


\begin{tabular}{||c|r|c|c||}
\hline gas & temp $(\mathrm{K})$ & $\Delta$ Hads $(\mathrm{cal})$ & area $\left(\mathrm{m}^{2}\right)$ \\
\hline xenon & 166 & 619 & 14.2 \\
xenon & 148 & 624 & 14.6 \\
xenon & 144 & 616 & 14.4 \\
\hline nitrogen & 77 & 709 & 15.7 \\
\hline
\end{tabular}

Table A.1: Nitrogen and xenon isotherms on polyacrylic acid. Surface areas and adsorption energies.

\section{A.3 Polyacrylic Isotherms}

The xenon isotherms on polyacrylic acid show characteristic BET behavior. In Figure A.2 three xenon isotherms are shown at 144, 148, and $166 \mathrm{~K}$ with BET curves illustrating the best fits to the data.

The results tabulated for the xenon and nitrogen isotherms are shown in the Table A.1. As one can see, the derived surface areas are slightly smaller than those for the nitrogen surface area measurement, but the adsorption energies for xenon are relatively constant. In order to use the isotherms in our work on polyacrylic acid, the values of the surface areas were averaged for the three isotherms to determine a value for $\mathrm{V}_{m o n}$, and a value of $c$ was calculated using equation (A.2) for each of the temperatures used in the NMR experiments. Knowing $p_{\circ}$ from saturated vapor pressure measurements, [232], we used equation (A.1) to calculate the coverage $\theta$, which is $\mathrm{V} / \mathrm{V}_{\text {mon }}$. We could therefore estimate the xenon coverages relatively accurately without having to do an isotherm at each of the experimental temperatures. 


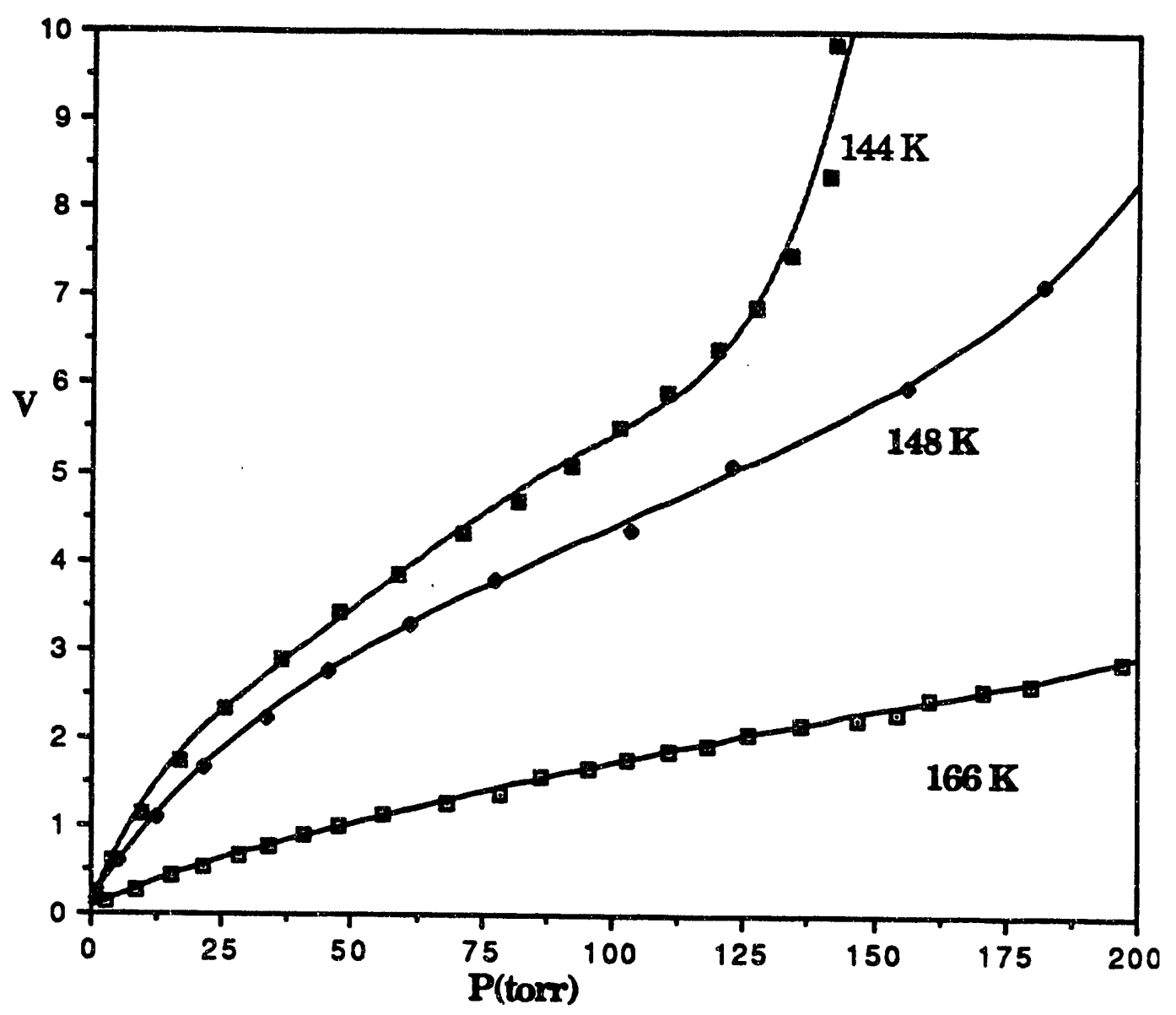

Figure A.2: BET isotherms of xenon on polyacrylic acid at 144,148, and $166 \mathrm{~K}$. 


\section{Appendix B}

\section{Low Field Experimental Details}

This appendix deals with some of the technical aspects of the low field experiment that were not discussed in Chapter 7 for reasons of brevity. The low field experiment is essentially a "home built" optical-NMR spectrometer which incorporates many of the features of modern NMR spectrometers except that it operates at radio frequencies which are low $(1-1000 \mathrm{kHz})$ by most NMR standards. The optical pumping and optical detection methods used in the experiments allow for high sensitivity and offset the fact that NMR signals usually scale with the frequency as $\omega^{p}$ where $p \simeq 2.5$ [233]. A block diagram of the important parts of the apparatus was shown in Figure 6.4. A more detailed discussion of the design now follows.

\section{B.1 Pulse Programmer}

To accurately control the timing of the experiment (since personal computers often contain wait states), a dedicated pulse programmer was constructed on a board to reside in an expansion slot of the PC. The pulse programmer card was based on a simple design from the University of Illinois [234]. This design featured a set of FIFO (first in first out) memory chips with 1024 words in depth and 24 bits wide 
to serve as the pulse programmer timing and gating words. We used 8 bits as the digital word output to control gates and the remaining 16 bits as timing words. The PC first loads the FIFO memory in Hexidecimal words with timing and gating words through one of its output ports and then starts the pulse programmer. The pulse programmer loads a gate word onto its output register and then clocks down the timing word using its onboard $1 \mathrm{MHz}$ clock. The gate word stays at the output until a new gate word is loaded and so most pulse programs end with a $\emptyset \emptyset$ Hexidecimal word used to clear the ouput. A block diagram of the important pulse programmer parts appears in Figure B.1. Pulse programs are also written in Hexidecimal and consist of a series of timing and gate words. Turbo pascal programs in the PC are used to load and start the pulse programmer.

\section{B.2 The Magnet}

The solenoid magnet design was based on an overwound design [191] consisting of two 236-turn layers of 10 Gauge square (for easier winding) copper wire plus two overwound layers of 27 turns each at the ends, wound on a rolled copper sheet $30 \mathrm{~cm}$ in diameter and $100 \mathrm{~cm}$ long. The number of turns in the overlayers was determined by computer calculations and depended critically on the wire spacing (cross section) of the main section. The solenoid was originally designed to have a homogeneity of better than $1 \mathrm{ppm}$ over a volume of $100 \mathrm{~cm}^{3}$. However, a winding mistake occurred in one of the overlayers and the resulting homogeneity was only about $100 \mathrm{ppm}$. As a result, shim coils were required to compensate for this problem. They consisted of $x$, $y$ and $z$ gradients plus $z^{2}$ coils [235], and were $25 \mathrm{~cm}$ in diameter. A constant current source power supply [236] was used to control these shims. The field homogeneity was improved using the NMR signal from a water sample, and the resulting homogeneity 


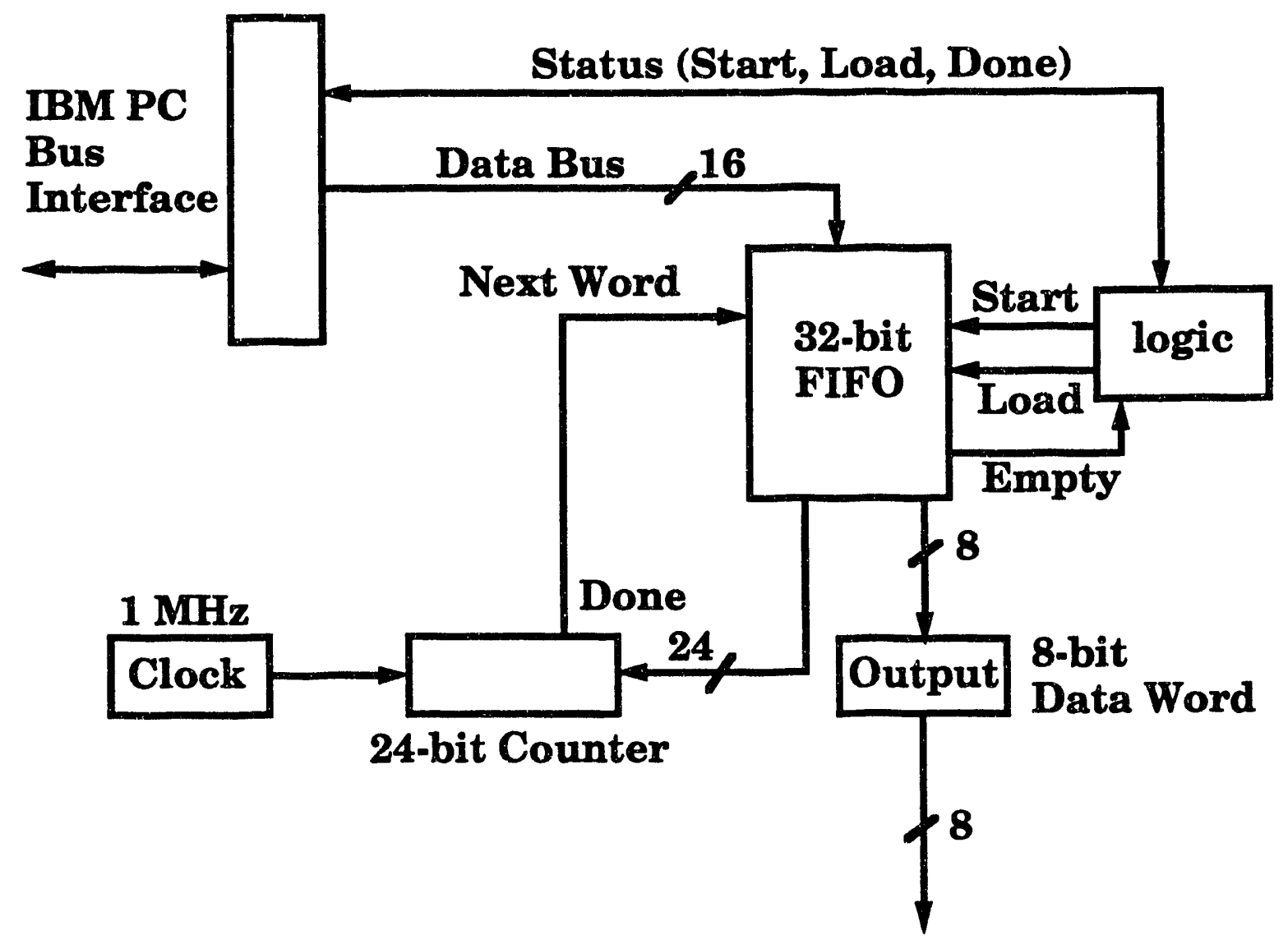

Figure B.1: Block diagram of the pulse programmer. 
was approximately $10 \mathrm{ppm}$.

\section{B.3 Magnet Supply}

The solenoid magnet power supply (Figure B.2) was constructed after a design by Z. Olejniczak. It consisted of a very stable current source using a nichrome wire sensing resistor in an oil temperature bath plus high stability components including an AD584 voltage source. The overall stability was approximately $10 \mathrm{ppm} /{ }^{\circ} \mathrm{C}$ that was primarily determined by the stability of the sensing resistor.

\section{B.4 Detector}

The detector was the primary noise determining element in the experiment and so considerable care was taken to reduce its effect. A schematic diagram is shown in Figure B.2. The detector consisted of a silicon photo detector, very low noise Linear Technologies (LT1024) bipolar operational amplifier in a transconductance configuration plus high stability resistors. Another feature of the design was the digital switches that allowed computcr control of the gain so that one could use the same detector to monitor the laser power during pumping. The voltage source for the detector consisted of two 9 volt batteries which were mounted in the same aluminum box as the detector electronics to reduce the problems associated with noise pickup.

The primary noise source is current noise in the detector plus voltage from the feedback resistor. The total noise in the detector system can be written [236]:

$$
\text { NoiseVoltage }=\sqrt{\left(I_{n} R\right)^{2}+V_{n}^{2}} .
$$

The shot noise, $I_{n}[236]$, is essentially the square root of the number of photo electrons. The best detector efficiency is realized by choosing $R$ such that $V_{n}=I_{n} R$. 

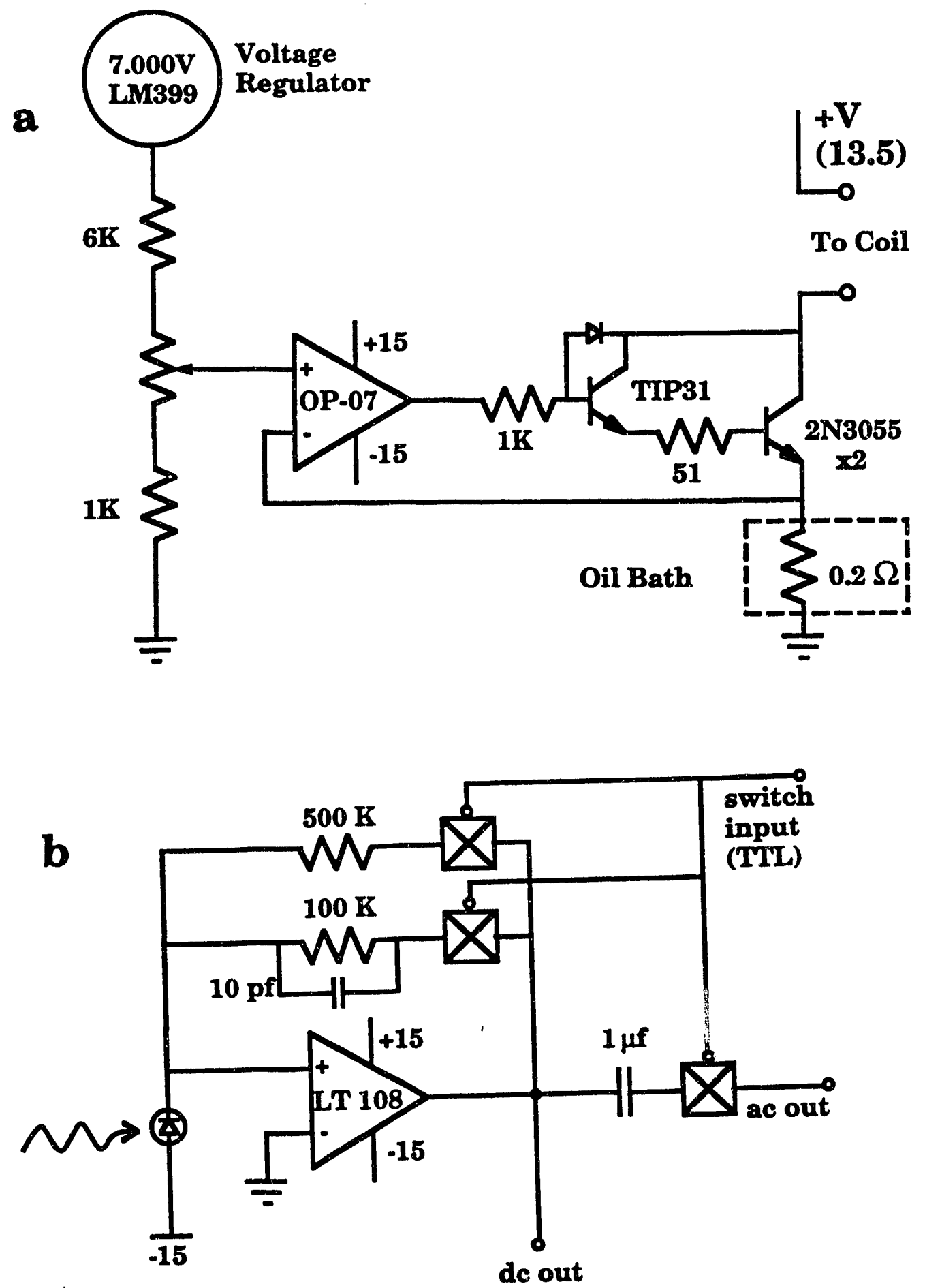

Figure B.2: (a) Solenoid magnet power supply. (b) Photo-detector circuit schematic. 


\section{B.5 Quadrature Generator}

In a normal NMR spectrometer, four phases of $\mathrm{r} \int$ radiation, $(x, y, \bar{x}, \bar{y})$ are generated using commercial quadrature splitter at some convenient intermodulation frequency such as $30 \mathrm{MHz}$. Shykind et al. [237] have recently published a design for a very accurate quadrature generator for use in demanding multiple pulse experiments where very accurate pulse phases and amplitudes are essential. Such components are very expensive at frequencies below $1 \mathrm{MHz}$. Following a suggestion by G. Chingas, an inexpensive quad box was constructed from TTL logic. Deriving synchronous pulses with the four phases is straight forward, as shown in Figure B.3.

A schematic diagram for the quad box and indicator box (to display the pulse output) is shown in Figure B.3(b). A multiplexer was used to chose the desired pulse phase. After selection, two 2-pule Bcssel filters and smoothed the square pulses to their fundamental frequency. A switch allowed one to select the cutoff frequency, 25 or $100 \mathrm{kHz}$.

\section{B.6 Rubidium Detection Lamp}

The rubidium lamp (Figure B.4) was based on a design by Brewer [192] and consisted of a vacuum tube resonator to generate an electrode-less discharge in the lamp bulb. Several other lamp designs exist [238], however most have problems with self-reversal [18] (see Figure B.5) where the emitted light spectrum has a hole in the middle due to rubidium in the outer portion of the bulb that absorbs the light emitted from the interior. Our larnp had severe problems with of radiation at its resonance frequency of $100 \mathrm{MH} \%$, a problem that could probably be reduced by using $\mu$-metal shielding common in other optically-detected low field experiments $[84,86]$. The lamp acted as a continuous $r f$ transmitter and has adverse effects on the magnet 
$\mathbf{a}$

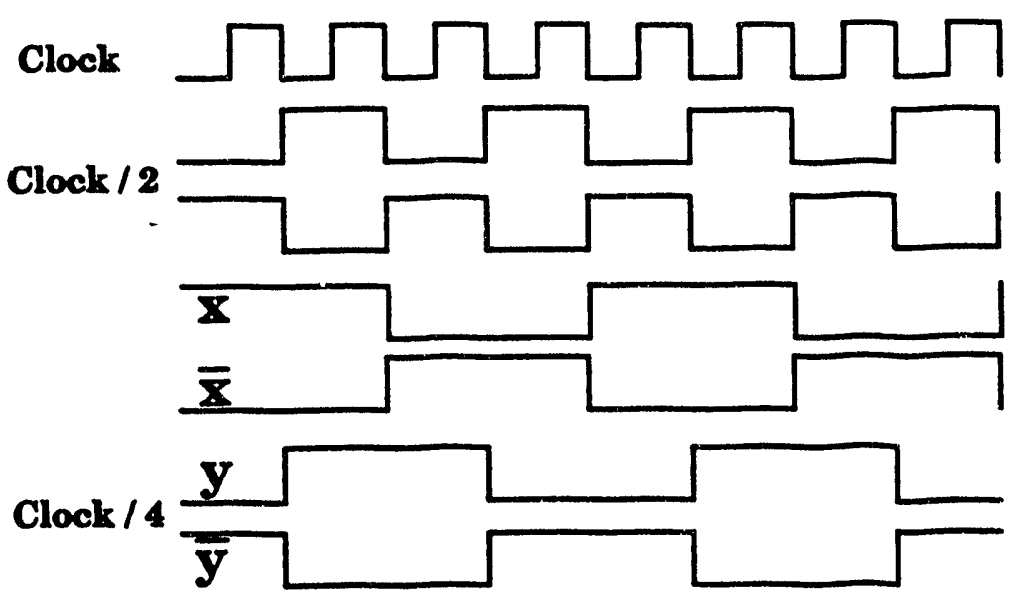

b
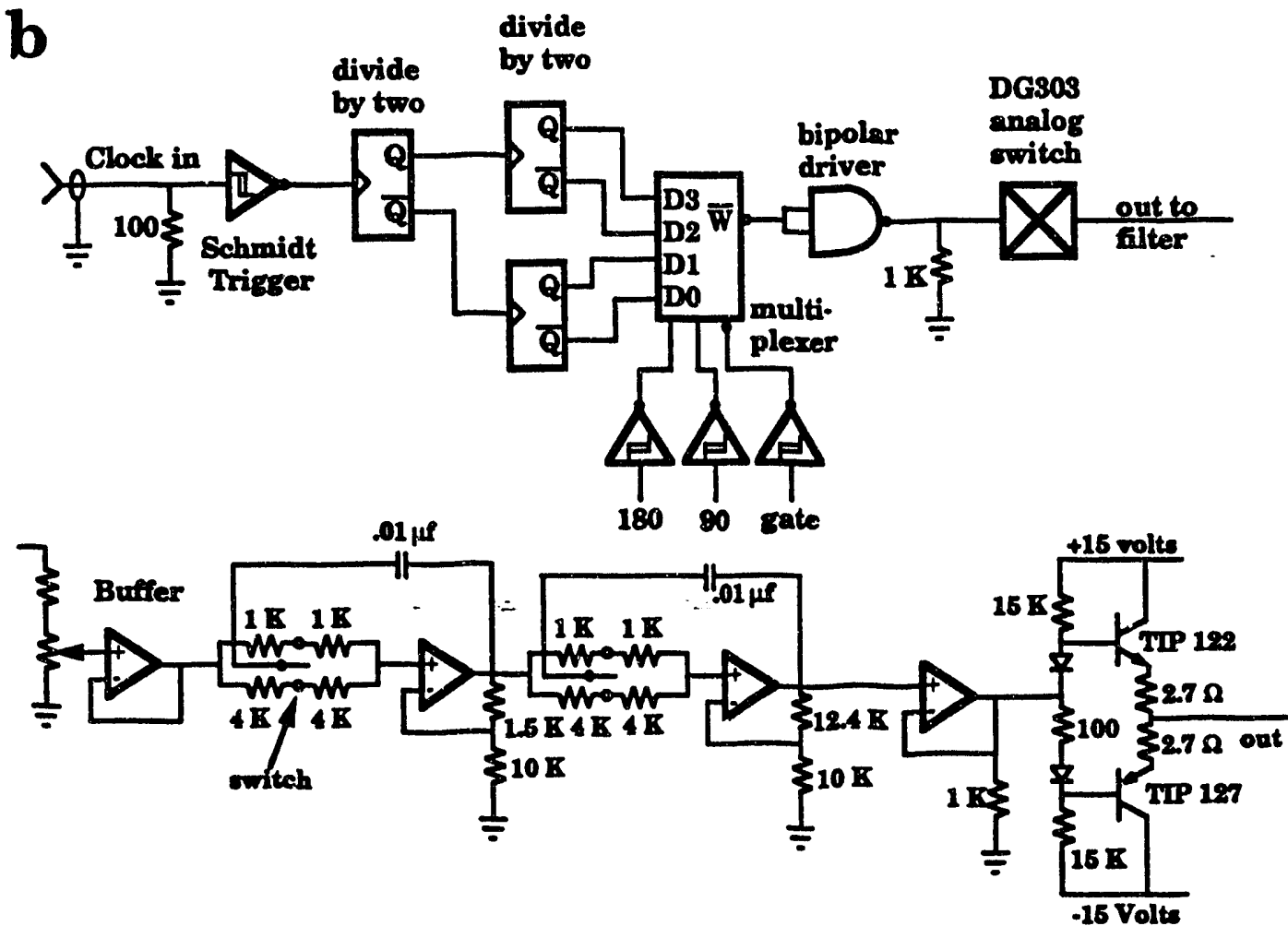

Figure B.3: (a) Four phase pulse generation using TTL. (b) Digital quadrature generation, filtering and display electronics. 


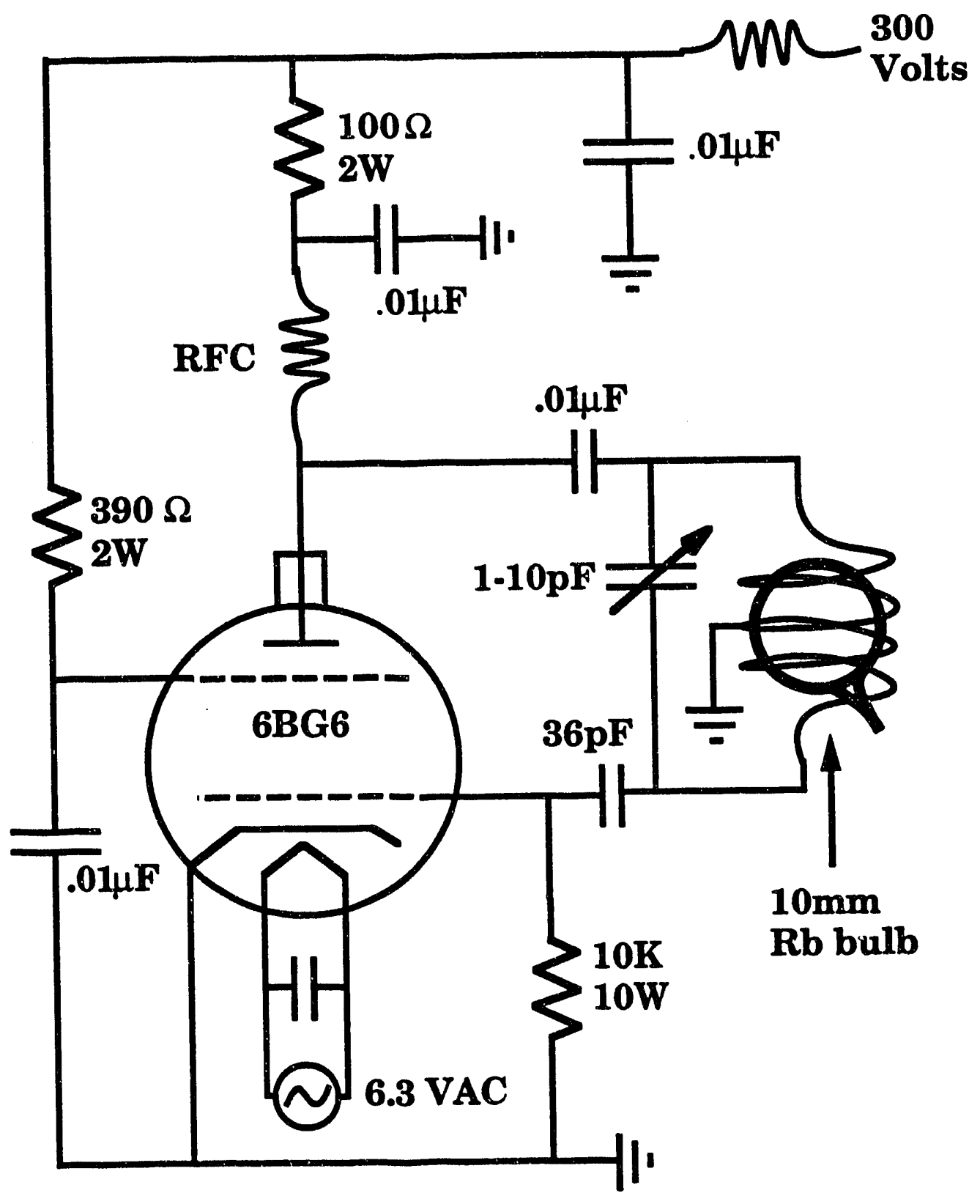

Figure B.4: Rubidium resonance lamp circuit. Adapted from Brewer [192]. 
power supply. Bypass capacitors and shielding (in a 1/4" aluminium box) helped to reduc 3 this problem. 


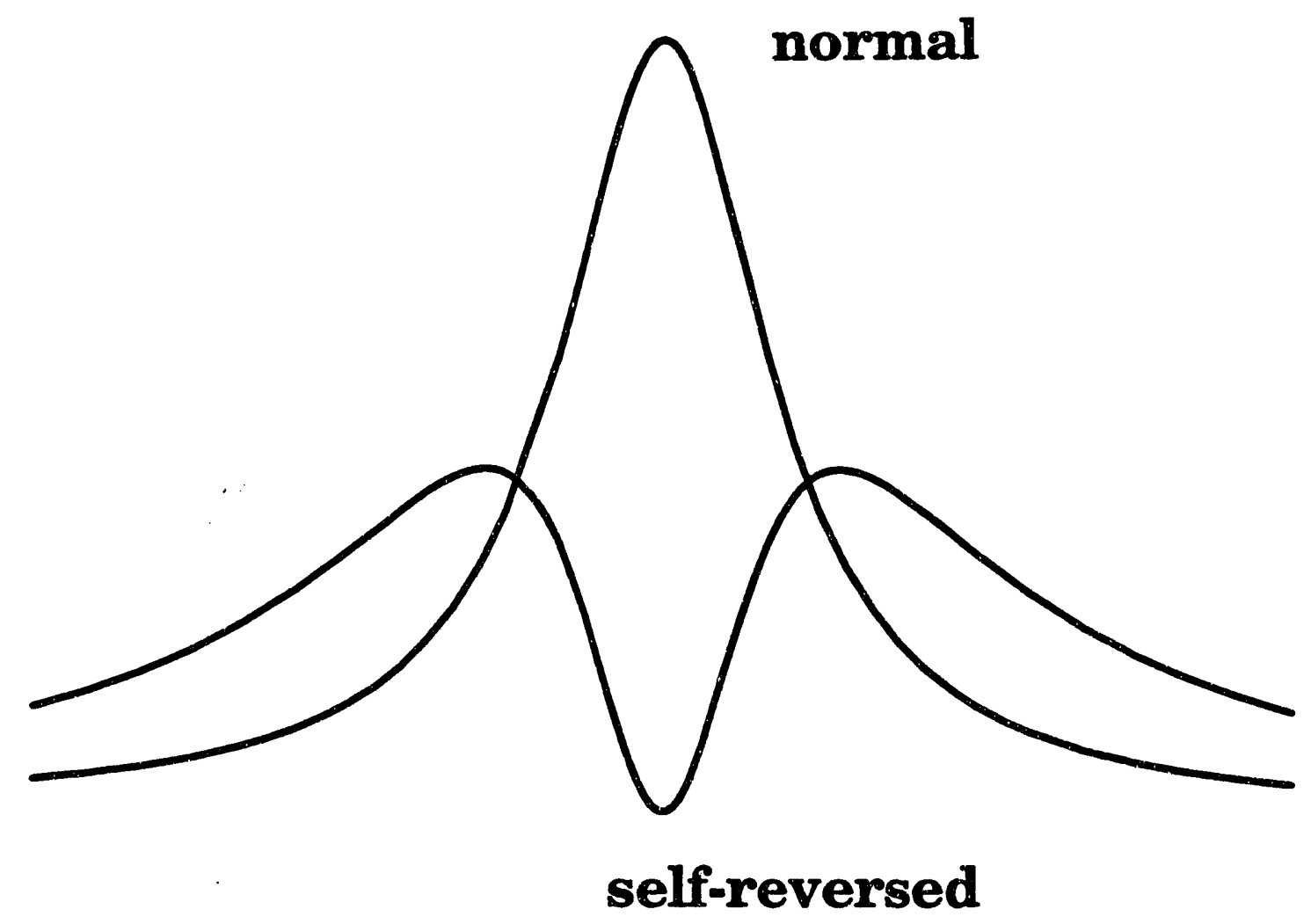

Figure E.5: Rubidium resonance lamp idealized output and self reversed output. Adapted from Bernheim [19] 


\section{Bibliography}

[1] G. J. Schrobilgen, "The Noble Gases," in NMR and the Periodic Table, R. K. Harris and B. E. Mann, ed. (Academic Press, London 1978).

[2] T. Ito and J. Fraissard, J. Phys. Chem. 76, 5225 (1982).

[3] J. A. Ripmeester, J. Am. Chem. Soc. 104, 289 (1982).

[4] P. J. Barrie and J. Klinowski, Prog. in NMR Spec. (in press).

[5] D. Raftery, H. Long, T. Meersmann, P. J. Grandinetti, L. Reven and A. Pines, Phys. Rev. Lett. 66, 584 (1991).

[6] N. F. Ramsey, Nuclear Moments, (J. Wiley, New York 1953); ibid, Phys. Rev. 77, 567 (1950); 78, 699 (1950) .

[7] W. Lamb, Phys. Rev. 60, 817 (1941).

[8] G. Malli and C. Froese, Int. J. Quan. Chem. 1S, 95 (1967).

[9] C. J. Jameson and H. S. Gutowski, J. Chem. Phys. 40, 1714 (1964).

[10] R. L. Streever and H. Y. Carr, Phys. Rev. 121, 20 (1961).

[11] A. K. Jameson, C. J. Jameson and H. S. Gutowski, J. Chem. Phys. 53, 2310 (1970). 
[12] R. Grosse, R. Burmeister, B. Boddenberg, A. Gedeon, and J. Fraissard, J. Phys. Chem. 95, 2443 (1991).

[13] C. P. Slichter, Principles of Magnetic Resonance, 3rd ed., (Springer-Verlag, Berlin, Germany 1990).

[14] N. Bloembergen, Nuclear Magnetic Relaxation, (W. A. Benjamin, New York 1961).

[15] A. Abragam, The Principles of Magnetic Resonance, (Clarendon, Oxford 1961).

[16] S. R. Hartmann and E. L. Hahn, Phys. Rev. 128, 2042 (1962); A. Pines, M. G. Gibby and J. S. Waugh, J. Chem. Phys. 59, 569 (1973).

[17] J. Brossel and A. Kastler, Comp. Rend. 229, 1213 (1949); A. Kastler, J. Phys. Radium 11, 255 (1950).

[18] T. R. Carver, Science 141, 599 (1963).

[19] R. Bernheim, Optical Pumping, (W. A. Benjamin, New York 1965).

[20] B. C. Grover, Phys.Rev. Lett. 40, 391 (1978).

[21] R. J. Knize, Z. Wu and W. Happer, in Adv. Atom. and Mol. Phys., (Academic Press, New York 1988), Vol. 24; W. Happer, Rev. Mod. Phys. 44, 169 (1972).

[22] C. C. Bouchiat, M. A. Bouchiat and L. C. Pottier, Phys. Rev., 181, 144 (1969); M. A. Bouchiat, J. Brossel and L. C. Pottier, J. Phys. Chem. 56, 3703 (1972).

[23] S. Appelt, P. Scheufler and M. Mehring, Opt. Comm., 74, 110 (1989).

[24] D. Suter, M. Rosatzin and J. Mlynek, Phys. Rev. Lett. 67, 34 (1991). 
[25] D. Suter and J. Mlynek, in Adv. Mag. and Opt. Res., (Academic Press, New York 1991), Vol. 16.

[26] W. Happer, E. Miron, S. Schaefer, W. A. van Wijngaarden and X. Zeng, Phys. Rev. A 29, 3092 (1984).

[27] R. M. Herman, Phys. Rev. 137A, 1062 (1965).

[28] N. D. Bhaskar, W. Happer, M. Larsson and X. Zeng, Phys. Rev. Lett. 50, 105 (1983).

[29] S. Haroche and C. Cohen-Tannoudji, Phys. Rev. Lett. 24, 974 (1970).

[30] S. Haroche, C. Cohen-Tannoudji, C. Audoin and J. P. Schermann, Phys. Rev. Lett. 24, 861 (1970).

[31] C. Cohen-Tannoudji, J. Phys. (Paris), 24, 653 (1963).

[32] X. Zeng, Z. Wu, T. Call, E. Miron, D. Schreiber and W. Happer, Phys. Rev. A $31260,(1985)$.

[33] C. H. Volk, T. M. Kwon and J. G. Mark, Phys. Rev. A 21, 1549 (1980).

[34] Hinds International Inc., Hillsboro, Oregon 97123.

[35] S. G. Redsun, R. J. Knize, G. D. Cates and W. Happer, Phys. Rev. A 42, 1293 (1990); S. G. Redson, Ph. D. Thesis, Princeton University, 1990.

[36] W. G. Proctor and F. C. Wu, Phys. Rev. 81, 20 (1951); E. Brun, J. Oeser, H. H. Staub and C. G. Telschow, Phys. Rev. 93, 904 (1954); ibid, Helv. Phys. Acta 27, 174 (1954).

[37] C. J. Jameson, A. K. Jameson and S. M. Cohen, J. Phys. Chem. 59, 4540 (1973). 
[38] F. J. Adrian, Phys. Rev. 136A, 980 (1964).

[39] C. J. Jameson, J. Chem. Phys. 63, 5296 (1975).

[40] J. A. Barker, R. O. Watts, J. K. Lee, T. P. Schaefer and Y. T. Lee, J. Chem. Phys. 61, 3081 (1974).

[41] E. Kanesberg, B. Pass and H. Y. Carr, Phys. Rev. Lett. 23, 572 (1969).

[42] F. J. Adrian, Chem. Phys. Lett. 7, 201 (1970).

[43] E. R. Hunt and H. Y. Carr, Phys. Rev. 130, 2302 (1963).

[44] H. C. Torrey, Phys. Rev. 130, 2306 (1963).

[45] B. Shizgal, Chem. Phys. 5, 464 (1974).

[46] J. A. Courtney and R. L. Armstrong, Can. J. Phys. 50, 1252 (1972); C. J. Jameson and A. K. Jameson, J. Chem. Phys. 88, 7448 (1988); C. J. Jameson, A. K. Jameson, J. K. Hwang and D. Dabkowski, J. Phys. Chem. 92, 5937 (1988).

[47] F. J. Adrian, Phys. Rev. 138, A403 (1965).

[48] D. Brinkmann, E. Brun and H. H. Staub, Helv. Phys. Acta 35, 431 (1962).

[49] C. J. Jameson, A. K. Jameson and S. M. Cohen, J. Chem. Phys. 62, 4224 (1975).

[50] ibid, J. Chem. Phys. 65, 3401 (1976).

[51] ibid, J. Chem. Phys. 66, 5226 (1977).

[52] C. J. Jameson, A. K. Jameson and H. Parker, J. Chem. Phys. 68, 394 (1978). 
[53] C. J. Jameson and A. K. Jameson, Mol. Phys. 20, 957 (1971); C. J. Jameson, A. K. Jameson and S. M. Cohen, J. Chem. Phys. 65, 3397 (1976).

[54] A. D. Buckingham and P. A. Kollman, Mol. Phys. 23, 65 (1972).

[55] C. J. Jameson, A. K. Jameson and S. M. Cohen, Mol. Phys. 29, 1919 (1975)

[56] W. M. Yen and R. E. Norberg, Phys. Rev. 131, 269 (1963).

[57] D. Brinkmann and H. Y. Carr, Phys. Rev. 150, 174 (1966).

[58] W. W. Warren, Jr. and R. E. Norberg, Phys. Rev. 148, 402 (1966).

[59] D. F. Cowgill and R. E. Norberg, Phys. Rev. B 6, 1636 (1972).

[60] J. Lurie, J. Feldman and G. Horton, Phys. Rev. 150, 180 (i964).

[61] J. Van Kronendonk, Physica 20, 781 (1954).

[62] W. W. Warren, Jr. and R. E. Norberg, Phys. Rev. 154, 227 (1967).

[63] L. M. Stacey, B. Pass and H. Y. Carr, Phys. Rev. Lett. 23, 1424, (1969); C. E. Hayes and H. Y. Carr, Phys. Rev. Lett. 39, 1558 (1977).

[64] A. Thomy and X. Duval, J. Chim. Phys. 67, 1101 (1970); J. Régnier, A. Thomy and X. Duval, J. Chim. Phys. 74, 926 (1977).

[65] W. Hong, C. J. Peters, A. Mak, R. J. Birgeneau, P. M. Horn and H. Suematsu, Phys. Rev. B 40, 4797 (1989); H. Hong and R. J. Birgeneau, Z. Phys. B Condensed Matter 77, 413 (1989).

[66] T. Shibanuma, H. Asada, S. Ishi and T. Matsui, Jpn. J. Appl. Phys., Part 1, 22, 1656 (Eng.) (1983).

[67] G. Neue, Z. Phys. Chem. Neue Folge, Bd. 152, 271 (Eng.) (1987). 
[68] J. D. Dash, Physics Today, p. 26, (Dec. 1985).

[69] Union Carbide, Allentown, PA.

[70] D. Raftery, H. Long, L. Reven, A. Pines, P. Tang and J. Reimer, (to be published).

[71] CRC Handbook of Chemistry and Physics, (CRC Press, Boca Raton, FL 1990), p. E-133.

[72] B. F. Chmelka, D. Raftery, A. V. McCormick, L. C. de Menorval, R. D. Levine, and A. Pines, Phys. Rev. Lett. 66, 580 (1991).

[73] M. G. Samant, L. C. de Menorval, R. A. Dalla-Betta, and M. Boudart, J. Phys. Chem. 92, 3937 (1988): J. A. Ripmeester and C. I. Radcliffe, J. Phys. Chem. 94, $7652(1990)$.

[74] B. F. Chmelka, D. Raftery, A. V. McCormick, L. C. de Menorval, R. D. Levine, and A. Pines, Phys. Rev. Lell. 67, 931 (1991).

[75] K. A. Brownlee, Statistical Theory and.Methodology (Wiley, New York 1965).

[76] J. Güémez and S. Velasco, Am. J. Phys. 55, 154 (1987).

[77] J. S. Rowlinson and G. B. Woods, Physica A (Amsterdam) 164, 117 (1990).

[78] G. D. Cates, D. R. Benton, M. Gatzke, W. Happer, K. C. Hasson and N. R. Newbury, Phys. Rev. Lett. 55, 2591 (1990).

[79] N. D. Bhaskar, W. Happer and T. McClelland, Phys. Rev. Lett. 49, 25 (1982).

[80] T. E. Chupp and M. E. Wagshul, K. P. Coulter, A. B. McDonald and W. Happer, Phys. Rev. C 36, 2244 (1987). 
[81] S. R. Schaefer, G. D. Cates, T.-R. Chien, D. Gonatas, W. Happer, and T. G. Walker, Phys. Rev. A 39, 5613 (1989).

[82] W. D. Knight, "Electron Paramagnetism and Nuclear Magnetic Resonance in Metals," in Solid State Physics, Vol. 2, F. Seitz and D. Turnvull, ed. (Academic Press, New York, 1956).

[83] T. M. Kwon, J. G. Mark and C. H. Volk, Phys. Rev. A 24, 1894 (1981).

[84] Z. Wu, W. Happer and J. M. Daniels, Phys. Rev. Lett. 59, 1480 (1987).

[85] Pierce Chemical Co., Rockford, IL 61105.

[86] C. H. Volk, T. M. Kwon, J. G. Mark, Y. B. Kim and J. C. Woo, Phys. Rev. Lett. 44, 136 (1980).

[87] C. Cohen-Tannoudji, Ph. D. Thesis, Paris 1962, in Ann. Phys. 7, pp. 423-460, pp. 469-504.

[88] Z. Wu, S. Schaefer, G. D. Cates, and W. Happer, Phys. Rev. A 371161 (1988).

[89] unpublished results.

[90] D. W. Breck, Zeolite Molecular Sieves, (Krieger, Malabar, FL 1982); R. M. Barrer, Zeolites and Clay Minerals as Sorbents and Molecular Sieves, (Academic Press, New York 1978).

[91] P. Michiels and O. C. E. de Herdt, Molecular Sieve Catalysts, Pergamon Press, Oxford 1987).

[92] J. Fraissard and T. Ito, Zeoliles 8, 350 (1988).

[93] T. Ito, L. C. de Menorval, E. Gucrrier and J. Fraissard, Chem. Phys. Lett. 111, $271(1984)$. 
[94] T. Ito and J. Fraissard, Zeolites 7, 554 (1987).

[95] T. T. P. Cheung, C. M. Fu and S. Wharry, J. Phys. Chem. 92, 5170 (1988).

[96] J. Demarquay and J. Fraissard, Chem. Phys. Lett. 136, 314 (1987).

[97] E. G. Derouane and J. B. Nagy, Chem. Phys. Lett. 137, 341 (1987).

[98] J. Fraissard, T. Ito, M. A. Springer-Huet and J. Demarquay, "Adsorption of Xenon: A New Method for Studying Zeolites," in New Developments in Zeolite Science and Technology, Ed. Y. Murakami, A. Iijima and J. W. Ward, ed. Stud. Surf. Sci. Catal. 28, 393 (1986).

[99] Q. J. Chen, M. A. Springer-Huet and J. Fraissard, Chem. Phys. Lett. 159, 117 (1989).

[100] M. A. Springer-Huet an?. J. Fraissard Chem. Phys. Lett., 154, 299 (1989).

[101] J. A. Ripmeester, C. I. Ratcliffe and J. S. Tse, J. Chem. Soc., Faraday Trans. f 84, 3731 (1988).

[102] L. Petrakis, M. A. Springer-Huet, T. Ito, T. R. Hughes, I. Y. Chan and J. Fraissard, "Assessment of Platinum Dispersion in Zeolites by ${ }^{129}$ Xe NMR and Comparison with TEM and Chemisorption," in 8th Int. Congress on Catalysis, Calgary, Canada (1988), pp. 348-355.

[103] B. F. Chmelka, R. Ryoo, S.-B. Liu, L. C. de Menorval, C. J. Radke, E. E. Petersen, and A. Pines, J. Am. Chem. Soc. 110, 4465 (1988).

[104] B. F. Chmelka, Ph. D. Thesis, University of California, Berkeley CA (1989). 
[105] L. C. de Menorval, J. Fraissard and T. Ito, J. Chem. Soc., Faraday Trans. 1 78, 403 (1982); L. C. de Menorval, J. Fraissard, T. Ito and M. Primet, J. Chem. Soc., Faraday Trans. 181,2855 (1985) .

[106] C. Tsiao, D. Corbin and C. Dybowski, J. Phys. Chem. 94, 867 (1990).

[107] T. Ito and J. Fraissard, J. Chim. Phys. 83, 441 (1986).

[108] Q. C. Chen and J. Fraissard, Chem. Phys. Lett. 169, 595 (1990).

[109] R. Shoemaker and T. Apple, J. Phys. Chem. 91, 4024 (1987).

[110] E. W. Scharpf, R. W. Crecely, B. C. Gates and C. Dybowski, J. Phys. Chem. 90, 9 (1986).

[111] A. Gedeon, J. L. Bonardet, T. Ito and J. Fraissard, J. Phys. Chem. 93, 2563 (1989).

[112] T. T. P. Cheung, J. Phys. Chem. 93, 7549 (1989).

[113] T. Ito and J. Fraissard, J. Chim. Phys. 83, 441 (1986).

[114] T. T. P. Cheung, J.- Catat. 124, 511 (1990).

[115] W. Heink, J. Kärger, H. Pfeifer and F. Stallmach, J. Am. Chem. Soc. 112, 2175 (1990); J. Kärger, H. Pfeifer, F. Stallmach and H. Spindler, Zeolites 10, 288 (1990).

[116] E. D. Stejskal and J. E. Tanner, J. Chem. Phys. 42, 288 (1965).

[117] J. Kärger, J. Mag. Res. 93, 184 (1991).

[118] S. D. Pickett, A. K. Nowak, J. M. Thomas, B. K. Peterson, J. F. P. S:vift, A. K. Cheetham, C. J. J. den Ouden, B. Smit and M. F. Post, J. Chem. Phys. 94, 1233 (1990). 
[119] J. Fraissard and J. Kärger, Zeolites 9, 351 (1989).

[120] N. Bansal and C. Dybowski, J. Mag. Res. 89, 21 (1990).

[121] T. Ito, J. L. Bonardet, J. Fraissard, J. B. Nagy, C. André, Z. Babelica and E. G. Derouane, Appl. Catal. 43, L5 (1988).

[122] J. T. Miller, B. L. Meyers and G. J. Ray, J. Catal. 128, 436 (1991).

[123] L. C. de Menorval, D. Raftery, S.-B. Liu, K. Takegoshi, R. Ryoo and A. Pines, J. Phys. Chem. 94, 27 (1990).

[124] B. F. Chmelka, J. G. Pearson, S.-B. Liu, R. Ryoo, L. C. de Menorval and A. Pines, J. Phys. Chem. 95, 303 (1991).

[125] R. Ryoo, s.-B. Liu, L. C. de Menorval, K. Takegoshi, B. Chmelka, M. Trecoske and A. Pines, J. Phys. Chem. 91, 6575 (1987).

[126] A. Pines, in Proceedings of the 100th School of Physics "Enrico Fermi,"; B. Maraviglia, ed. (North-Holland, Amsterdam 1988).

[127] D. J. Suh, T.-J. Park, S.-K. Ihm and R. Ryoo, J. Phys. Chem. 95, 3767 (1991).

[128] C. Tsiao and R. E. Botto, Energy and Fuels 5, 87 (1991).

[129] P. C. Wernett, J. W. Larsen, O. Yamada and H. J. Yue, Energy and Fuels 4, 412 (1990).

[130] J. Jokisaari and P. Diehl, Liq. Cryst. 7, 739 (1990).

[131] P. Diehl and J. Jokisaari, J. Mag. Res. 88, 660 (1990).

[132] P. Diehl and J. Jokisaari, Chem. Phys. Lett. 165, 389 (1990). 
[133] T. R. Stengle, N. V. Reo and K. L. Williamson, J. Phys. Chem. 88, 3225 (1984).

[134] H. G. Hertz, Ben. Bunsenges Phys. Chem. 77, 531, 688 (1973); ibid, 78, 24 (1984).

[135] F. H. A. Rummens, in van der Waals Forces and Shielding Effects, NMR Basic Principles and Progress Vol. 10 (Springer-Verlag, New York, 1975).

[136] T. R. Stengle, S. M. Hosseini, H. G. Basiri and K. L. Williamson, J. Sol. Chem. 13, 779 (1984).

[137] J. A. Ripmeester, J. S. Tse and C. I. Ratclifle, Nature 325, 135 (1987).

[138] J. A. Ripmeester and C. I. Ratcliffe, J. Phys. Chem. 94, 8773 (1990).

[139] D. W. Davidson, Y. P Handa and J. A. Ripmeester, J. Phys. Chem. 90, 6549 (1986).

[140] M. Mehring, Principles of High Resolution NMR in Solids, 2nd ed. (Springer, Berlin 1983).

[141] C. P. Slichter, W. C. Holton, Phys. Rev. 122, 1701 (1961).

[142] A. Pines and T. W. Shattuck, Chem. Phys. Lett. 23614 (1973).

[143] J. A. Ripmeester and D. W. Davidson, J. Mol. Struc. 75, 67 (1981).

[144] J. A. Ripmeester, C. I. Radcliffe and J. S. Tse, J. Chem. Soc. Farad. Trans. 1 84,3731 (1988).

[145] T. R. Stengle and K. R. Williamson, Macromolecules 20, 1430 (1987). 
[146] S. K. Brownstein, J. E. L. Roovers and D. J. Worsfold, Mag. Res. in Chem. 26, 392 (1988).

[147] G. Kennedy, Poly. Bull. 23, 605 (1990).

[148] N. Bartlett, Proc. Chem. Soc. 218, (1982).

[149] T. H. Brown, E. B. Whipple and P. H. Verdier, J. Chem. Phys. 38, 3029 (1963); ibid, Noble Gas Compounds, H. H. Hyman, ed. (Univ. of Chicago Press, Chicago 1963).

[150] K. Seppelt and H. H. Rupp, Z. Anorg. Chem 409, 331 and 338 (1974).

[151] G. J. Schrobilgen, J. H. Holloway, R. Granger and C. Brevard, Inorg. Chem. 17,980 (1978).

[152] J. H. Holloway and G. J. Schrobilgen, Inorg. Chem. 19, 2632 (1980).

[153] J. F. Sawyer, G. J. Schrobilgen and S. J. Sutherland, J. Chem. Soc. Commun., 210 (1982).

[154] D. Naumann and W. Tyrra, J. Chem. Soc., Chem. Commun. 47 (1989).

[155] L. Turowsky and K. Seppelt, Inorg. Chem. 29, 3226 (1989).

[156] K. W. Miller, N. V. Reo, A. J. M. Schoot Uiterkamp, D. P. Stengle, T. R. Stengle and K. L Williamson, Proc. Nat. Acad. Sci. USA 78, 4946 (1981).

[157] R. F. Tilton, Jr. and I. D. Kuntz, Biochemistry 21, 6850 (1982).

[158] T. Ito, L. C. de Menorval and J. Fraissard, J. Chim. Phys. 80, 573 (1983).

[159] A. Guideon, T. Ito and J. Fraissard, Zeolites 8, 376 (1988). 
[160] H. Lechert and K.-P. Wittern, Ber. Bunsenges. Phys. Chem. 82, 1024 (1978); H. Lechert, W. D. Basler and K.-P. Wittern, "Nuclear Relaxation Studies of Aromats in Faujasite Type Zeolite," in Proceedings of the 7th International Zeolite Conference, Tokyo, 1986, (Elsevier, Oxford 1986).

[161] J. Fraissard, Z. Phys. Chem. 152, 159 (1987).

[162] A. de Mallmann, and D. Barthmeuf, "Four Different Studies of Benzene Adsorbed in Faujasites," in Proceedings of the 7th International Zeolite Conference, Tokyo, 1986 (Elsevier, Oxford 1986) pp. 609-615.

[163] J. Fraissard, private communication.

[164] J. Güémez, S. Velasco, and A. C. Hernández, Physica A 152, 226 and 243 (1988).

[165] G. B. Woods, A. Z. Panagiotopoulos, and J. S. Rowlinson, Mol. Phys. 63, 49 (1988).

[166] D. W. Cooper, Phys. Rev. A38, 522 (1988).

[167] A. V. Kiselev and P. Q. Du, J. Chem. Soc., Faraday Trans. 2, 77, 1 (1981).

[168] J. J. Magda, M. Tirell and H. T. Davis, J. Chem. Phys. 83, 1888 (1985).

[169] T. K. Vanderlick, L. E. Scriven and H. T. Davis, J. Chem. Phys. 90, 2422 (1989).

[170] K. Jameson, private communication.

[171] M. G. Samant, L. C. de Menorval, R. A. Dalla-Betta and M. Boudart, J. Phys. Chem. 92, 3937 (1988). 
[172] J. A. Ripmeester and C. I. Ratcliffe, J. Phys. Chem. 94, 7652 (1990).

[173] A. V. McCormick and B. F. Chmelka, Mol. Phys., 603 (1991).

[174] W. H. Press, B. P. Flannery, S. A. Teukolsky and W. T. Vetterling, Numerical Recipes in Pascal, (Cambridge University Press, Cambridge 1988).

[175] J. Fraissard, T. Ito, L. C. de Menorval, and M. A. Spinguel-Huet, in Metal Microstructures in Zeolites, P. ^. Jacobs el al., ed. (Elsevier, Amsterdam, 1982).

[176] E. Cohen De Lara, R. Kahn, and A. M. Goulay, J. Chem. Phys. 90, 7482 (1989).

[177] T. R. Rybolt, R. L. Mitche!l, and C. M. Waters, Langmuir 3, 326 (1987).

[178] R. A. Aziz and M. J. Slaman, Mol. Phys. 57, 825 (1986).

[179] D. M. Ruthven, Principles of Adsorption and Adsorption Processes (Wiley, New York, 1984).

[180] P. van Tassel, H. T. Davis, and A. V. McCormick, Mol. Phys., submitted.

[181] Z. Wu, W. Happer, M. Kitano and J. Daniels, Phys. Rev. A 42, 2774 (1990).

[182] T. E. Chupp and R. J. Hoare, Phys. Rev. Lett. 64, 2261 (1990).

[183] M. H. Levitt, Prog. NMR Speclros. 18, 61 (1986); A. J. Shaka and J. Keeler, Adv. Mag. Reson. 11, 48 (1983).

[184] T. Killian, Phys. Rev. 27, 578 (1926).

[185] R. L. Gamblin and T. R. Carver, Phys. Rev. A 138, 946 (1965); L. D. Schearer and G. K. Walters, Phys. Rev. A 139, 1398 (1965).

[186] E. L. Hahn, Phys. Rev. 80, 580 (1950). 
[187] H. Y. Carr and E. M. Purcell, Phys. Rev. 94, 630 (1954).

[188] R. M. Sternheimer, Phys. Rev. 105, 158 (1957).

[189] P. K. Majumder, B. J. Venema, S. K. Lamereaux, B. R. Heckel and E. N. Fortson, Phys. Rev. Lett. 65, 2931 (1990).

[190] G. Bahm, Lectures of Quantum Mechanics, (Benjamin/Cummings Publishing Co., London 1969).

[191] M. W. Garrett, J. Appl. Phys. 38, 2563 (1967).

[192] R. G. Brewer, Rev. Sci. Instr. 32, 1356 (1961).

[193] R. L. Strombotne and E. L. Hahn, Fhys. Rev. A, 133, 1616 (1964); R. E. Slusher and E. L. Hahn, Phys. Rev. 166, 332 (1'968).

[194] D. P. Weitekamp, A. Bielecki, D. Zax, K. Zilm and A. Pines, Phys. Rev. Lett. 50, 1807 (1983); D. B. Zax, A. Bielecki, K. W. Zilm, A. Pines and D. P. Weitekamp, J. Chem. Phys. 83, 4877 (1985).

[195] M. H. Levitt, R. Freeman and T. Frenkiel, J. Mag. Res. 50, 157 (1982).

[196] R. R. Ernst, G. Bodenhausen and A. Wokaun, Principles of Nuclear Magnetic Resonance in One and Two Dimensions, (Clarendon, Oxford 1987).

[197] S. Meiboom and D. Gill, Rev. Sci. Instr. 29,6881 (1958).

[198] A. T. Nicol, Phys. Rev. B 29, 2397 (1984).

[199] A. T. Nicol, "Nuclear Moment Alignment, Relaxation and Dztection," Air Force Technical Report AFOSR-TR-82-0418, (1982).

[200] Z. Liu, X. Sun, X. Zeng and Q. He, Chin. Phys. Lett. 7, 399 (1990)., 
[201] A. Fines, "NMR in Physics, Chemistry, and Biology: Illustrations of Bloch's Legacy" in Proceedings of the Bloch Symposium, W. A. Little, ed. (World Scientific, Singepore 1990).

[202] V. Lefevre-Seguin and J. Brossel, J. Low Temp. Phys. 72, 165 (1988).

[203] T. E. Chupp, R. J. Hoare, R. A. Loveman, E. R. Oteiza, J. M. Richardson, M. E. Wagshul, and A. K. Thompson, Phys Rev. Lett. 63, 1541 (1989).

[204] M. Kitano, M. Bourzutschky, F. P. Calaprice, J. Clayhold, W. Happer, and M. Musolf, Phys. Ren. C 34, 1974 (1986).

[205] M. A. Springuel-Huet and J. Fraissard, Chem. Phys. Lett. 154, 299 (1989); T. Ito and J. Fraissard, Zeolites 8, 350 (1988); E. W. Scharpf, R. W. Creceley, B. C. Gates, and C. Dybowski, J. Phys. Chem. 90, 9 (1986); M. E. Davis, C. Saldarriaga, C. Montes, and B. Hanson, J. Phys. Chem. 92, 2557 (1988).

[206] B. F. Chmelka, L. C. de Menorval, R. Csencsits, R. Ryoo, S.-B. Liu, C. J. Radke, E. E. Petersen, and A. Pines, in Structure and Reactivity of Surfaces, C. Morterra, et al., ed. (Elsevier, Amsterdam, 1989).

[207] O. Gonen, P. L. Kuhns, J. S. Waugh, and J. P. Fraissard, J. Phys. Chem. 93, 504 (1989).

[208] C. P. Slichter, Ann. Rev. Phys. Chem. 37, 25 (1986).

[209] C. J. Jameson, A. K. Jameson, and J. K Hwang, J. Chem. Phys. 89, 4074 (1988).

[210] F. D. Doty, R. R. Inners and P. Ellis, J. Mag. Res. 43, 399 (1981).

[211] P. W. Atkins, Physical Chemistry, 3rd ed., (W. H. Freeman and Co., New York 1986). 
[212] J. Tabony and T. Cosgrove, Chem. Phys. Lett. 67, 103 (1979).

[213] M. Krapf, G. Denninger, H. Pascher, G. Weimmann, et al., Solid State Comm. 78, 459 (1991); ibid, Superlatlices and Microstructures 8, 91 (1990).

[214] R. Superfine, J. Y. Huang and Y. R. Shen, Phys. Rev. Lett. 66, 1066 (1991); X. Zhao, S. Subrahmanyan and K. B. Eisenthal, Chem. Phys. Lett. 171, 558 (1990).

[215] M. S. Pettersen and D. L. Goodstein, Surf. Sci. 209, 455 (1989).

[216] G. Tastevin, P. J. Nacher, L. Wiesenfeld, M. Leduc and F. Laloë, J. Phys. France 49, 584 (1988).

[217] S. C.-K. Chu, Y. Xu, J. A. Balschi and C. S. Springer, Jr., Mag. Res. in Medicine, 239 (1978).

[218] D. W. Davies, The Theory of the Electric and Magnetic Properties of Molecules, (J. Wiley, New York 1967).

[219] J. R. Zimmerman and M. R. Forster, J. Phys. Chem. 61, 282 (1957).

[220] K. G. Orrell and V. Šik, Anal. Chem. 52, 567 (1980).

[221] U. Haberlen, High Resolution NMR in Solids: Selective Averaging, (Academic Press, New York 1976).

[222] E. C. Stoner, Phil. Mag. 36, 803 (1945).

[223] S. J. Opella and J. S. Waugh, J. Chem. Phys. 66, 4919 (1977).

[224] N. Bloernbergen and T. J. Rowland, Phys. Rev. 97, 1679 (1955).

[225] H. Y. Carr and E. M. Purcell, Phys. Rev. 94, 630 (1954). 
[226] G. Malli and C. Froese, Int. J. Quan. Chem. 1S, 90 (1967).

[227] C. Barter, R. G. Meisenheimer and D. P. Stevenson, J. Phys. Chem. 64, 1312 (1960).

[228] J. R. Packard and C. A. Swenson, J. Phys. Chem. Solids 24, 1405 (1963).

[229] D. Brinkmann, Phys. Rev. Lelt. 13, 187 (1964).

[230] A. W. Adamson, Physical Chemistry of Surfaces, (J. Wiley, New York 1982).

[231] S. Brunauer, P. H. Emmctt and E. Teller, J. Am. Chem. Soc. 60, 309 (1938).

[232] M. P. Freeman and G. D. Halsley, Jr., J. Phys. Chem. 60, 1119 (1956);G. A. Cook, Argon, Helium and the Rare Gases, (Interscience, New York 1961), Vol. 1.

[233] D. I. Hoult and R. E. Richards, J. Mag. Res. 24, 71 (1976); F. D. Doty, T. J. Connick, X. Z. Ni and M. N. Clingan, J. Mag. Res. 77, 536 (1988).

[234] C. Hawley, Dept. of Chern., Univcrsity of Illinois, private communication.

[235] M. J. E. Golay, Rev. Sci. Instr. 29, 313 (1958); ibid, U. S. Patent Nos. 3,569,823 and $3,622,869$.

[236] P. Horowitz and W. Hill, The Art of Electronics, (Cambridge University Press, Cambridge 1980).

[237] D. N. Shykind, G. C. Chingas, A. Pines and A. J. Shaka, Rev. Sci. Instr. 61, 1474 (1990).

[238] W. F. Bell, A. L. Bloom and J. Lynch, Rev. Sci. Instr. 32, 688 (1961); F. A. Franz Phys. Rev. 127, 589 (1963). 

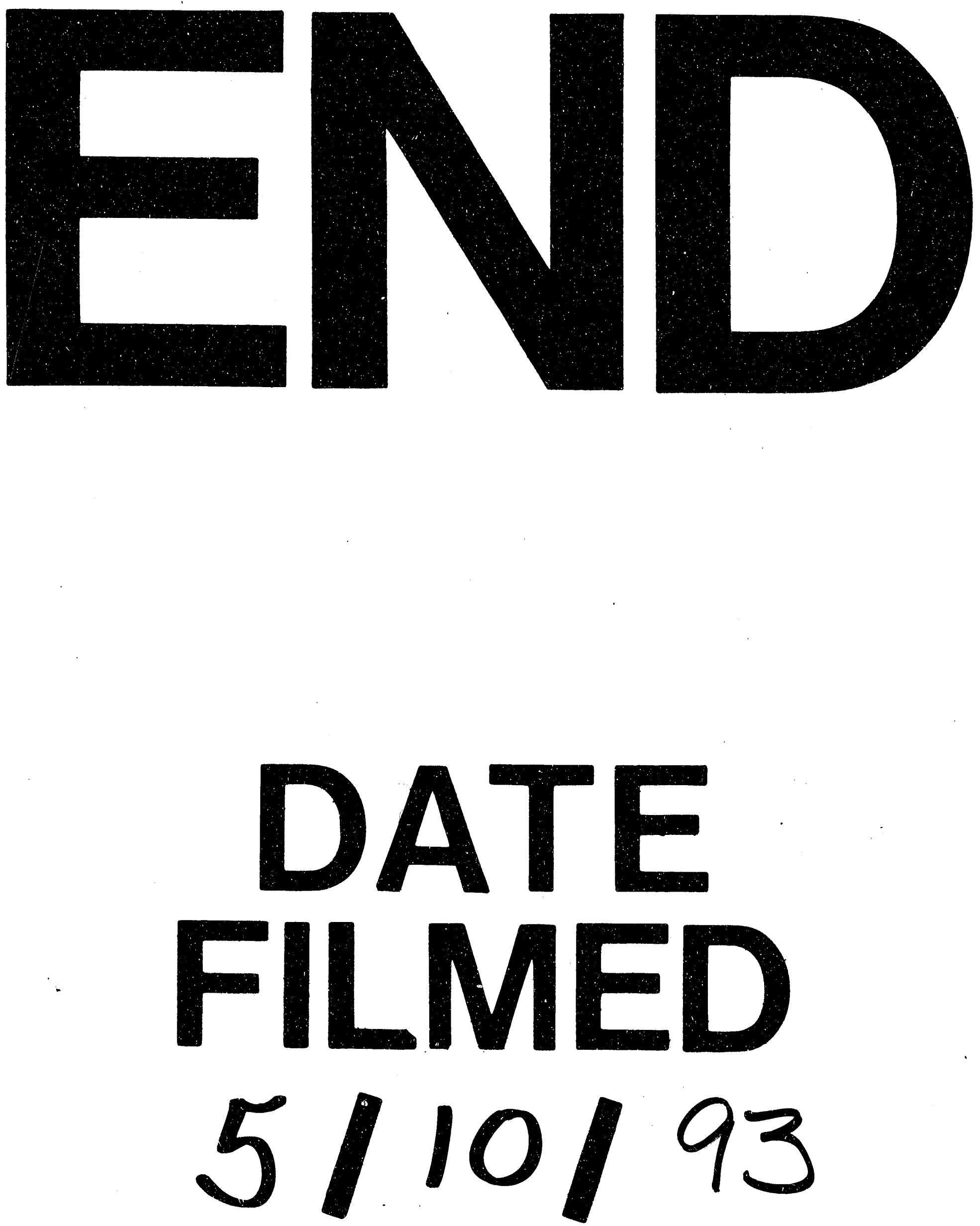

93 
